\title{
Water-Quality, Bed-Sediment, and Biological Data (October 2010 through September 2011) and Statistical Summaries of Data for Streams in the Clark Fork Basin, Montana
}

Banner: Metal salts on the surface of tailings deposit near Silver Bow at Opportunity (12323600) gage (photograph by Kent A. Dodge, U.S. Geological Survey, taken May 2012).

Cover: Looking upstream at the confluence of Silver Bow Creek and Warm Springs Creek, which form the Clark Fork near Warm Springs, Montana (photograph by Kent A. Dodge, U.S. Geological Survey, taken May 2012). 


\section{Water-Quality, Bed-Sediment, and \\ Biological Data (October 2010 through September 2011) and Statistical Summaries of Data for Streams in the Clark Fork Basin, Montana}

By Kent A. Dodge, Michelle I. Hornberger, and Jessica L. Dyke

Prepared in cooperation with the U.S. Environmental Protection Agency

Open-File Report 2013-1017 


\title{
U.S. Department of the Interior SALLY JEWELL, Secretary
}

\author{
U.S. Geological Survey \\ Suzette M. Kimball, Acting Director
}

U.S. Geological Survey, Reston, Virginia: 2013

For more information on the USGS - the Federal source for science about the Earth, its natural and living resources, natural hazards, and the environment, visit http://www.usgs.gov or call 1-888-ASK-USGS.

For an overview of USGS information products, including maps, imagery, and publications, visit http://www.usgs.gov/pubprod

To order this and other USGS information products, visit http://store.usgs.gov

Any use of trade, product, or firm names is for descriptive purposes only and does not imply endorsement by the U.S. Government.

Although this report is in the public domain, permission must be secured from the individual copyright owners to reproduce any copyrighted materials contained within this report.

Suggested citation:

Dodge, K.A., Hornberger, M.I., and Dyke, J.L., 2013, Water-quality, bed-sediment, and biological data (October 2010 through September 2011) and statistical summaries of data for streams in the Clark Fork basin, Montana:

U.S. Geological Survey Open-File Report 2013-1017, 132 p. 


\section{Contents}

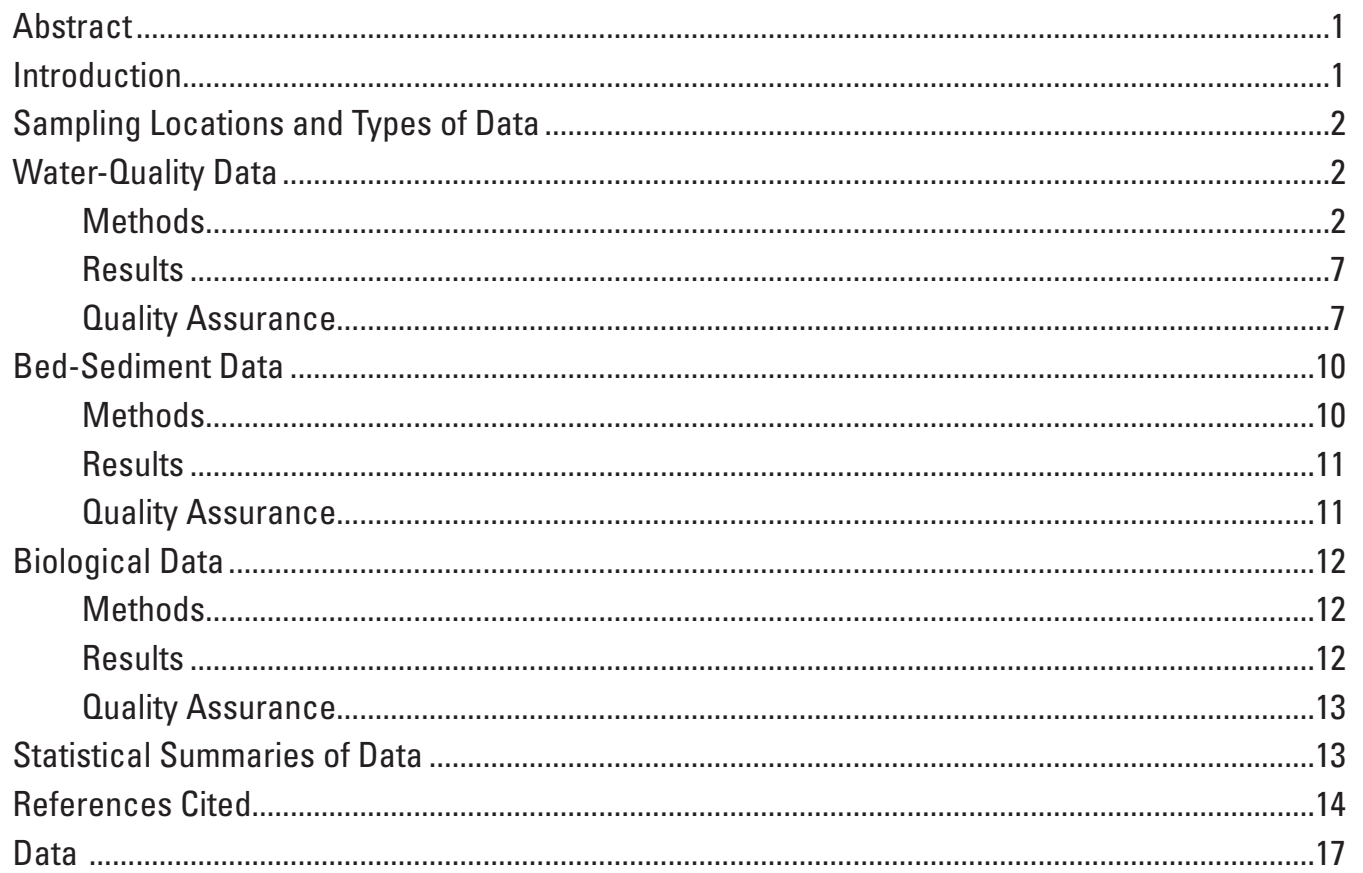

\section{Figure}

1. Map showing location of study area in the Clark Fork basin, Montana

\section{Tables}

1. Type and period of data collection at sampling sites in the Clark Fork basin, Montana

2. Properties and constituents measured onsite or analyzed in water, bed-sediment, and biota samples from the Clark Fork basin, Montana .............................

3. Data-quality objectives for analyses of water samples collected in the Clark Fork basin, Montana

4. Water-quality data for the Clark Fork basin, Montana, October 2010 through September 2011

5. Daily mean streamflow and suspended-sediment data for Clark Fork at Deer Lodge, Montana, October 2010 through September 2011..

6. Daily mean streamflow and suspended-sediment data for Clark Fork at Turah Bridge, near Bonner, Montana, October 2010 through September 2011

7. Daily mean streamflow and suspended-sediment data for Blackfoot River near Bonner, Montana, October 2010 through September 2011 
8. Daily mean streamflow and suspended-sediment data for Clark Fork above Missoula, Montana, October 2010 through September 2011

9. Seasonal daily maximum, minimum, and mean turbidity, with monthly summary statistics at Mill Creek near Anaconda, Montana, April through September 2011.

10. Seasonal daily maximum, minimum, and mean turbidity, with monthly summary statistics at Willow Creek near Anaconda, Montana, May through September 2011.

11. Seasonal daily maximum, minimum, and mean turbidity, with monthly summary statistics at Warm Springs Creek near Anaconda, Montana, April through September 2011

12. Seasonal daily maximum, minimum, and mean turbidity, with monthly summary statistics at Lost Creek near Anaconda, Montana, April through September 2011.

13. Analyses of field replicates for water samples, Clark Fork basin, Montana ...................70

14. Precision of analyses of field replicates for water samples, Clark Fork basin, Montana

15. Precision of analyses of laboratory replicates for water samples, Clark Fork basin, Montana

16. Recovery efficiency for analyses of laboratory-spiked deionized-water blank samples

17. Recovery efficiency for analyses of laboratory-spiked stream samples, Clark Fork basin, Montana

18. Analyses of field blanks for water samples ..................................................................

19. Bed-sediment data for the Clark Fork basin, Montana, August 2011 ..............................79

20. Recovery efficiency for analyses of standard reference materials for bed-sediment samples.

21. Analyses of procedural blanks for bed-sediment samples ..........................................81

22. Biological data for the Clark Fork basin, Montana, August 2011 _..................................82

23. Recovery efficiency for analyses of standard reference material for biota samples

24. Analyses of procedural blanks for biota samples...........................................................

25. Statistical summary of long-term water-quality data for the Clark Fork basin, Montana, March 1985 through September 2011.

26. Statistical summary of long-term bed-sediment data for the Clark Fork basin, Montana, August 1986 through August 2011. 106

27. Statistical summary of long-term biological data for the Clark Fork basin, Montana, August 1986 through August 2011 


\section{Conversion Factors, Datum, Abbreviated Water-Quality Units, and Acronyms}

\begin{tabular}{lcl}
\hline \multicolumn{1}{c}{ Multiply } & By & \multicolumn{1}{c}{ To obtain } \\
\hline acre-foot (acre-ft) & 1,233 & cubic meter $\left(\mathrm{m}^{3}\right)$ \\
cubic foot per second $\left(\mathrm{ft}^{3} / \mathrm{s}\right)$ & 0.02832 & cubic meter per second $\left(\mathrm{m}^{3} / \mathrm{s}\right)$ \\
gallon (gal) & 3.785 & liter $(\mathrm{L})$ \\
gallon (gal) & 3,785 & milliliter $(\mathrm{mL})$ \\
inch (in.) & 25.4 & millimeter $(\mathrm{mm})$ \\
inch (in.) & 25,400 & micrometer $(\mu \mathrm{m})$ \\
mile (mi) & 1.609 & kilometer $(\mathrm{km})$ \\
ounce (oz) & 28.35 & gram $(\mathrm{g})$ \\
part per million (ppm) & 1 & microgram per gram $(\mu \mathrm{g} / \mathrm{g})$ \\
square mile (mi $\left.{ }^{2}\right)$ & 2.59 & square kilometer $\left(\mathrm{km}{ }^{2}\right)$ \\
ton & 907.2 & kilogram \\
ton per day (ton/d) & 907.2 & kilogram per day $(\mathrm{kg} / \mathrm{d})$ \\
\hline
\end{tabular}

Temperature in degrees Celsius $\left({ }^{\circ} \mathrm{C}\right)$ may be converted to degrees Fahrenheit $\left({ }^{\circ} \mathrm{F}\right)$ as follows:

$$
{ }^{\circ} \mathrm{F}=\left(1.8 \mathrm{x}^{\circ} \mathrm{C}\right)+32
$$

Horizontal coordinate information is referenced to the North American Datum of 1927 (NAD 27).

\section{Water-year definition:}

Water year is the 12-month period from 0ctober 1 through September 30 of the following calendar year. The water year is designated by the calendar year in which it ends. For example, water year 2011 is the period from October 1, 2010, through September 30, 2011. 
Abbreviated water-quality units used in this report:

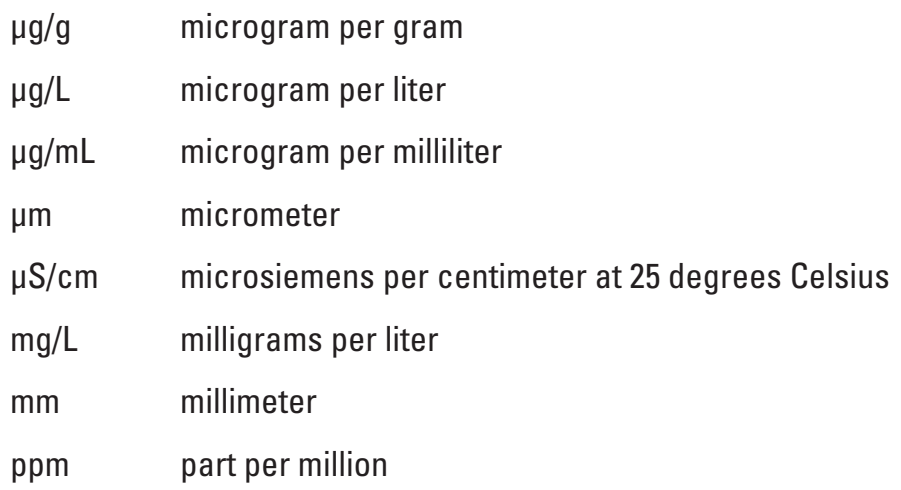

\section{Acronyms used in the report:}

$\begin{array}{ll}\text { FNU } & \text { formazin nephelometric units } \\ \text { ICP-AES } & \text { inductively coupled plasma-atomic emission spectrometry } \\ \text { ICP-MS } & \text { inductively coupled plasma-mass spectrometry } \\ \text { LRL } & \text { laboratory reporting level } \\ \text { LT-MDL } & \text { long-term method detection level } \\ \text { MRL } & \text { minimum reporting level } \\ \text { NTRU } & \text { nephelometric turbidity ratio unit } \\ \text { NWOL } & \text { USGS National Water Quality Laboratory, Denver, Colo. } \\ \text { PTFE } & \text { polytetrafluoroethylene } \\ \text { RSD } & \text { relative standard deviation } \\ \text { SPp. } & \text { species } \\ \text { SRM } & \text { standard reference material } \\ \text { USGS } & \text { U.S. Geological Survey } \\ \text { YSI } & \text { Yellow Springs Instruments Company }\end{array}$




\title{
Water-Quality, Bed-Sediment, and Biological Data (October 2010 through September 2011) and Statistical Summaries of Data for Streams in the Clark Fork Basin, Montana
}

\author{
By Kent A. Dodge, Michelle I. Hornberger, and Jessica L. Dyke
}

\section{Abstract}

Water, bed sediment, and biota were sampled in streams from Butte to near Missoula, Montana, as part of a monitoring program in the upper Clark Fork basin of western Montana; additional water samples were collected from near Galen to near Missoula at select sites as part of a supplemental sampling program. The sampling program was conducted by the U.S. Geological Survey in cooperation with the U.S. Environmental Protection Agency to characterize aquatic resources in the Clark Fork basin, with emphasis on trace elements associated with historic mining and smelting activities. Sampling sites were located on the Clark Fork and selected tributaries. Water samples were collected periodically at 20 sites from October 2010 through September 2011. Bedsediment and biota samples were collected once at 14 sites during August 2011.

This report presents the analytical results and qualityassurance data for water-quality, bed-sediment, and biota samples collected at sites from October 2010 through September 2011. Water-quality data include concentrations of selected major ions, trace elements, and suspended sediment. Turbidity was analyzed for water samples collected at the four sites where seasonal daily values of turbidity were being determined. Daily values of suspended-sediment concentration and suspended-sediment discharge were determined for four sites. Bed-sediment data include trace-element concentrations in the fine-grained fraction. Biological data include traceelement concentrations in whole-body tissue of aquatic benthic insects. Statistical summaries of water-quality, bedsediment, and biological data for sites in the upper Clark Fork basin are provided for the period of record since 1985 .

\section{Introduction}

The Clark Fork originates near the town of Warm Springs in western Montana at the confluence of Silver Bow and
Warm Springs Creeks (fig. 1). Along the 148-mile (mi) reach of stream from Silver Bow Creek in Butte to the Clark Fork near Missoula, six major tributaries enter: Blacktail Creek, Warm Springs Creek, Little Blackfoot River, Flint Creek, Rock Creek, and Blackfoot River. Principal surface-water uses in the 6,000-square-mile $\left(\mathrm{mi}^{2}\right)$ upper Clark Fork basin above Missoula include irrigation, stock watering, smallscale industry, and habitat for trout fisheries. Primary current land uses are cattle production, logging, mining, residential development, and recreation. Large-scale mining and smelting were prevalent land uses in the upper basin for more than 100 years but are now either discontinued or substantially reduced in scale.

Deposits of copper, gold, silver, and lead ores were extensively mined, milled, and smelted in the drainages of Silver Bow and Warm Springs Creeks from about the 1860s to the 1980s (U.S. Environmental Protection Agency, 2004). Moderate- and small-scale mining also occurred in the basins of most of the major tributaries to the upper Clark Fork. Tailings produced during past mineral processing commonly contain large quantities of trace elements such as arsenic, cadmium, copper, lead, and zinc. Eroded tailings mix with stream sediment and get deposited further downstream in stream channels, on flood plains, in the Warm Springs Ponds, and at the location of the former Milltown Reservoir (Milltown Dam was removed on March 28, 2008) (Andrews, 1987), especially during times of increased flow. The occurrence of elevated trace-element concentrations in water and bed sediment can pose a potential risk to aquatic biota and human health (U.S. Environmental Protection Agency, 2004).

Concern about the potential toxicity of trace elements to aquatic biota and human health has resulted in a comprehensive effort by State, Federal, Tribal, and private entities to characterize the aquatic resources in the upper Clark Fork basin in order to guide and monitor remedial cleanup activities. A long-term database was considered necessary to detect trends over time in order to evaluate the effectiveness of remediation. Water-quality data have been collected by the U.S. Geological Survey (USGS) at selected sites in the upper 
Clark Fork basin since 1985 (Lambing, 1987 through 1991; Lambing and others, 1994, 1995; Dodge and others, 1996 through 2010, 2012). Trace-element data for bed sediment and biota (aquatic benthic insects) have been collected intermittently at selected sites since 1986 as part of studies on the contamination of bed-sediment quality and bioaccumulation of metals conducted by the USGS National Research Program (Axtmann and Luoma, 1991; Cain and others, 1992, 1995; Axtmann and others, 1997; Hornberger and others, 1997). In March 1993, an expanded monitoring program for water, bed sediment, and biota in the upper basin was implemented by the USGS in cooperation with the U.S. Environmental Protection Agency to systematically quantify the seasonal and annual variability in selected constituents.

The purpose of this report is to present water-quality data from samples collected at 20 sites and bed-sediment and biological data from samples collected at 14 sites in the Clark Fork basin from October 2010 through September 2011. Quality-assurance data are presented for water-quality, bed-sediment, and biota samples collected during the same time period. Statistical summaries also are provided for waterquality, bed-sediment, and biological data collected at the sites since 1985 .

\section{Sampling Locations and Types of Data}

Sampling sites for the monitoring program in the upper Clark Fork basin from Butte to near Missoula (fig. 1) are located on the Clark Fork main stem (including Silver Bow Creek), three major tributaries (Blacktail Creek, Warm Springs Creek, and Blackfoot River), and three smaller tributaries (Mill Creek, Willow Creek, and Lost Creek). The sites, types of data collected, and period of record for each type of data are listed in table 1. Main-stem sampling sites were selected to divide the upper Clark Fork into reaches of relatively uniform length, with each reach encompassing either a major tributary or depositional environment (Warm Springs Ponds and the former Milltown Reservoir). Major tributaries were sampled to describe water-quality, bed-sediment, and biological characteristics of important hydrologic sources in the upper basin and to provide reference comparisons to the main stem. The three smaller tributaries were sampled to gain better spatial resolution on sources of metals entering the Clark Fork in an area of historical metal-processing activities near Anaconda, Mont. Water-quality samples were collected periodically at 20 sites. Daily suspended-sediment samples were collected at 4 sites, and daily turbidity values were computed using data collected by continuous turbidity monitors at 4 sites. Bedsediment and biological samples were collected once annually at 14 sites. Continuous streamflow data were collected at 19 sites.

Properties measured onsite and constituents for which water, bed-sediment, and biota samples were analyzed are listed in table 2. Data-quality objectives for analyses of water samples are listed in table 3. Results of onsite measurements of stream properties; laboratory analyses of water-quality, bed-sediment, and biota samples; and quality-assurance data for water year 2011 are listed in tables 4 through 24 at the back of the report. Statistical summaries of water-quality, bedsediment, and biological data collected between March 1985 and September 2011 are listed in tables 25 through 27 at the back of the report.

Quality assurance of data was maintained through the use of documented procedures designed to provide environmentally representative data. Acceptable performance of the procedures was verified with quality-control samples that were collected systematically to provide a measure of the accuracy, precision, and bias of the environmental data, and to identify problems associated with sampling, processing, or analysis.

\section{Water-Quality Data}

Water-quality data consist of onsite measurements of selected stream properties and concentrations of chemical and physical constituents analyzed in periodically collected stream samples. Routine water samples for the monitoring program were collected at 20 sites in the upper Clark Fork basin 6-8 times per year on a schedule designed to describe seasonal and hydrologic variability. Supplemental water samples were collected 4 additional times at 7 sites. At the 4 daily suspended-sediment sites, suspended-sediment samples were collected by an observer 1-11 times per week, depending on season and flow conditions. Continuous turbidity monitors operated seasonally (April or May to September 2011) at 4 sites near Anaconda collected turbidity values every 15 minutes that were used to compute daily turbidity values (table 1).

\section{Methods}

Water samples were collected and composited from vertical transits throughout the entire stream depth at multiple locations across the stream by using depth- and widthintegration methods described by Ward and Harr (1990), Edwards and Glysson (1999), and the U.S. Geological Survey (variously dated). These methods provide a vertically and laterally discharge-weighted composite sample that is intended to be representative of the entire flow passing through the cross section of a stream. Samplers consisted of depth-integrating water-quality samplers (Davis, 2005) that were constructed of plastic or coated with a nonmetallic rubber-coating paint and equipped with nylon or polytetrafluoroethylene (PTFE) nozzles.

Instantaneous streamflow was determined at the time of water sampling either by direct measurement or from stage-discharge rating tables (Rantz and others, 1982). Daily mean streamflow values during ice periods were estimated 

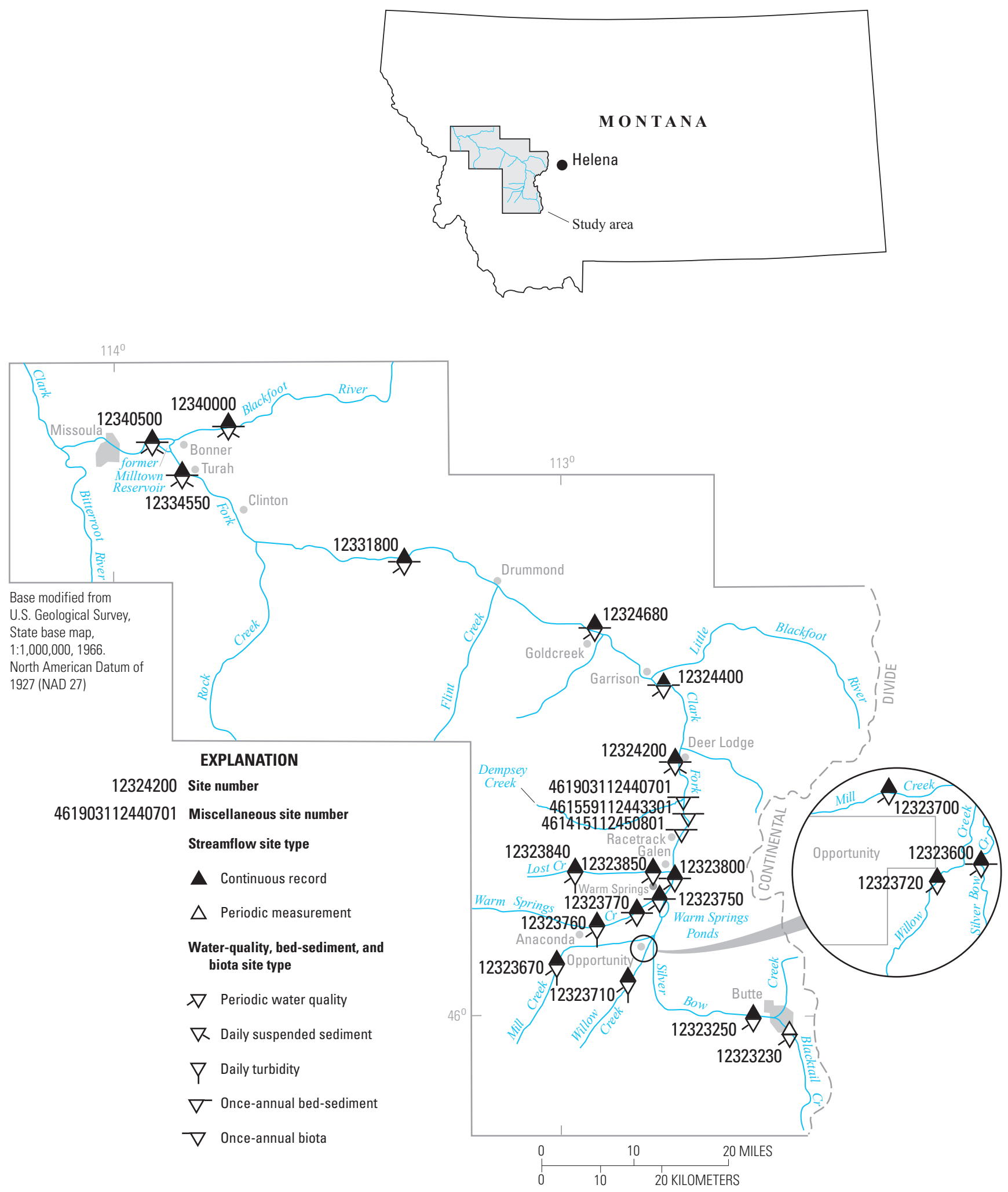

Figure 1. Location of study area in the Clark Fork basin, Montana. 
Table 1. Type and period of data collection at sampling sites in the Clark Fork basin, Montana.

[Abbreviations: P, present; D, discontinued. Symbol: --, no data]

\begin{tabular}{|c|c|c|c|c|c|c|c|c|}
\hline $\begin{array}{l}\text { Station number } \\
\text { (fig. 1) }\end{array}$ & Station name & $\begin{array}{l}\text { Continuous- } \\
\text { record } \\
\text { streamflow }\end{array}$ & $\begin{array}{c}\text { Periodic } \\
\text { water quality }\end{array}$ & $\begin{array}{l}\text { Daily suspended } \\
\text { sediment }\end{array}$ & $\begin{array}{l}\text { Daily turbidity } \\
\text { (seasonal) }\end{array}$ & $\begin{array}{l}\text { Fine-grained } \\
\text { bed sediment }^{2}\end{array}$ & $\begin{array}{l}\text { Bulk bed } \\
\text { sediment }\end{array}$ & Biota $^{2}$ \\
\hline 12323230 & $\begin{array}{l}\text { Blacktail Creek at Harrison } \\
\text { Avenue, at Butte }\end{array}$ & -- & $\begin{array}{c}03 / 93-08 / 95 \\
12 / 96-08 / 03 \\
12 / 04-\mathrm{P}\end{array}$ & -- & -- & -- & -- & -- \\
\hline 12323250 & $\begin{array}{l}\text { Silver Bow Creek below } \\
\text { Blacktail Creek, at Butte }\end{array}$ & 10/83-P & $\begin{array}{l}\text { 03/93-08/95 } \\
\text { 12/96-P }\end{array}$ & -- & -- & -- & -- & -- \\
\hline 12323600 & $\begin{array}{l}\text { Silver Bow Creek at } \\
\text { Opportunity }\end{array}$ & 07/88-P & $\begin{array}{l}\text { 03/93-08/95 } \\
\text { 12/96-P }\end{array}$ & 03/93-09/95, D & -- & 07/92-P & $\begin{array}{l}\text { 08/93-08/95, } \\
08 / 97-08 / 04, \mathrm{D}\end{array}$ & $\begin{array}{c}\text { 07/92, 08/94- } \\
08 / 95,08 / 97-\mathrm{P}\end{array}$ \\
\hline 12323670 & Mill Creek near Anaconda & 10/04-P & 12/04-P & -- & 06/06-P & -- & -- & -- \\
\hline 12323700 & Mill Creek at Opportunity & 04/03-P & 03/03-P & -- & -- & -- & -- & -- \\
\hline 12323710 & $\begin{array}{l}\text { Willow Creek near } \\
\text { Anaconda }\end{array}$ & 03/05-P & 12/04-P & -- & 06/06-P & -- & -- & -- \\
\hline 12323720 & $\begin{array}{l}\text { Willow Creek at } \\
\text { Opportunity }\end{array}$ & 04/03-P & 03/03-P & -- & -- & -- & -- & -- \\
\hline 12323750 & $\begin{array}{l}\text { Silver Bow Creek at Warm } \\
\text { Springs }\end{array}$ & $\begin{array}{c}03 / 72-09 / 79 \\
04 / 93-\mathrm{P}\end{array}$ & 03/93-P & 04/93-09/95, D & -- & 07/92-P & $\begin{array}{l}\text { 08/93, 08/95- } \\
\text { 08/04, D }\end{array}$ & 07/92-P \\
\hline 12323760 & $\begin{array}{l}\text { Warm Springs Creek near } \\
\text { Anaconda }\end{array}$ & 10/97-P & $10 / 05-\mathrm{P}$ & -- & 05/06-P & -- & -- & -- \\
\hline 12323770 & $\begin{array}{l}\text { Warm Springs Creek at } \\
\text { Warm Springs }\end{array}$ & 10/83-P & 03/93-P & -- & -- & $\begin{array}{c}08 / 95,08 / 97 \\
08 / 99,08 / 02 \\
08 / 05,08 / 08 \\
08 / 11\end{array}$ & $\begin{array}{l}\text { 08/95, 08/97, } \\
08 / 99,08 / 02, \mathrm{D}\end{array}$ & $\begin{array}{c}08 / 95,08 / 97 \\
08 / 99,08 / 02 \\
08 / 05,08 / 08 \\
08 / 11\end{array}$ \\
\hline 12323800 & Clark Fork near Galen & 07/88-P & 07/88-P & -- & -- & 08/87, 08/91-P & 08/93-08/04, D & 08/87, 08/91-P \\
\hline 12323840 & Lost Creek near Anaconda & 10/04-P & 12/04-P & -- & 05/06-P & -- & -- & -- \\
\hline 12323850 & Lost Creek near Galen & 04/03-P & 03/03-P & -- & -- & -- & -- & -- \\
\hline 461415112450801 & $\begin{array}{l}\text { Clark Fork below Lost } \\
\text { Creek, near Galen }\end{array}$ & -- & -- & -- & -- & 08/96-P & 08/96-08/04, D & 08/96-P \\
\hline 461559112443301 & $\begin{array}{l}\text { Clark Fork at county bridge, } \\
\text { near Racetrack }\end{array}$ & -- & -- & -- & -- & 08/96-P & 08/96-08/04, D & 08/96-P \\
\hline 461903112440701 & $\begin{array}{l}\text { Clark Fork at Dempsey } \\
\text { Creek diversion, near } \\
\text { Racetrack }\end{array}$ & -- & -- & -- & -- & 08/96-P & 08/96-08/04, D & 08/96-P \\
\hline
\end{tabular}


Table 1. Type and period of data collection at sampling sites in the Clark Fork basin, Montana.-Continued

[Abbreviations: P, present; D, discontinued. Symbol: --, no data].

\begin{tabular}{|c|c|c|c|c|c|c|c|c|}
\hline $\begin{array}{l}\text { Station number } \\
\text { (fig. 1) }\end{array}$ & Station name & $\begin{array}{l}\text { Continuous- } \\
\text { record } \\
\text { streamflow }\end{array}$ & $\begin{array}{c}\text { Periodic } \\
\text { water quality }\end{array}$ & $\begin{array}{l}\text { Daily suspended } \\
\text { sediment }\end{array}$ & $\begin{array}{l}\text { Daily turbidity } \\
\text { (seasonal) }\end{array}$ & $\begin{array}{l}\text { Fine-grained } \\
\text { bed sediment }^{2}\end{array}$ & $\begin{array}{l}\text { Bulk bed } \\
\text { sediment }\end{array}$ & Biota $^{2}$ \\
\hline 12324200 & Clark Fork at Deer Lodge & 10/78-P & $03 / 85-\mathrm{P}$ & $\begin{array}{c}03 / 85-08 / 86 \\
04 / 87-03 / 03 \\
08 / 03-P\end{array}$ & -- & $\begin{array}{c}\text { 08/86-08/87, } \\
\text { 08/90-P }\end{array}$ & 08/93-08/04, D & $\begin{array}{c}\text { 08/86-08/87, } \\
\text { 08/90-P }\end{array}$ \\
\hline 12324400 & $\begin{array}{l}\text { Clark Fork above Little } \\
\text { Blackfoot River, near Gar- } \\
\text { rison }\end{array}$ & 02/09-P & 03/09-P & -- & -- & 08/09-P & -- & 08/09-P \\
\hline 12324680 & Clark Fork at Goldcreek & 10/77-P & 03/93-P & -- & -- & 07/92-P & 08/93-08/04, D & 07/92-P \\
\hline 12331800 & Clark Fork near Drummond & 04/93-P & 03/93-P & -- & -- & $\begin{array}{c}08 / 86,08 / 87 \\
08 / 91-\mathrm{P}\end{array}$ & 08/93-08/04, D & 08/86, 08/91-P \\
\hline 12334550 & $\begin{array}{l}\text { Clark Fork at Turah Bridge, } \\
\text { near Bonner }\end{array}$ & 03/85-P & 03/85-P & $\begin{array}{c}03 / 85-03 / 03 \\
08 / 03-\mathrm{P}\end{array}$ & -- & 08/86, 08/91-P & 08/93-08/04, D & 08/86, 08/91-P \\
\hline 12340000 & Blackfoot River near Bonner & 10/39-P & 03/85-P & $\begin{array}{c}07 / 86-04 / 87 \\
06 / 88-09 / 95 \\
10 / 05-\mathrm{P}\end{array}$ & -- & $\begin{array}{c}\text { 08/86-08/87, } \\
\text { 08/91, } \\
08 / 93-08 / 96 \\
08 / 98-08 / 01 \\
\text { 09/03, } \\
\text { 08/06-P }\end{array}$ & $\begin{array}{c}\text { 08/93, 08/94 } \\
\text { 08/99-01 } \\
\text { 09/03, D }\end{array}$ & $\begin{array}{c}08 / 86-08 / 87 \\
08 / 91,08 / 93 \\
08 / 96,08 / 98 \\
09 / 00,09 / 03 \\
\text { 08/06-P }\end{array}$ \\
\hline 12340500 & Clark Fork above Missoula & 03/29-P & $07 / 86-\mathrm{P}^{4}$ & $\begin{array}{c}07 / 86-04 / 87 \\
06 / 88-01 / 96 \\
03 / 96-03 / 03 \\
08 / 03-P\end{array}$ & 04/07-09/07 & 08/97-P & 08/97-08/04, D & 08/97-P \\
\hline
\end{tabular}

${ }^{1}$ Onsite measurements of physical properties and laboratory analyses for selected major ions, trace elements, and suspended sediment. Prior to March 1993 , laboratory analyses included only trace elements and suspended sediment.

${ }^{2}$ Laboratory analyses for trace elements.

${ }^{3}$ Bulk bed-sediment sampling was discontinued in 2005 .

${ }^{4}$ Prior to October 1989, water-quality data for Clark Fork above Missoula included only suspended-sediment data. 
Table 2. Properties and constituents measured onsite or analyzed in water, bed-sediment, and biota samples from the Clark Fork basin, Montana.

\begin{tabular}{|c|c|c|c|}
\hline \multicolumn{2}{|c|}{ Water } & \multirow{2}{*}{$\begin{array}{c}\text { Bed sediment } \\
\text { Constituent }\end{array}$} & \multirow{2}{*}{$\begin{array}{c}\text { Biota } \\
\text { Constituent }\end{array}$} \\
\hline Property & Constituent & & \\
\hline Streamflow & Hardness (calculated) & Arsenic & Arsenic \\
\hline $\mathrm{pH}$ & Calcium & Cadmium & Cadmium \\
\hline Specific conductance & Magnesium & Chromium & Chromium \\
\hline Temperature & Arsenic & Copper & Copper \\
\hline \multirow[t]{7}{*}{ Turbidity } & Cadmium & Iron & Iron \\
\hline & Copper & Lead & Lead \\
\hline & Iron & Manganese & Manganese \\
\hline & Lead & Nickel & Nickel \\
\hline & Manganese & Zinc & Zinc \\
\hline & Zinc & & \\
\hline & Suspended sediment & & \\
\hline
\end{tabular}

Table 3. Data-quality objectives for analyses of water samples collected in the Clark Fork basin, Montana.

[Abbreviations: $\mu \mathrm{g} / \mathrm{L}$, micrograms per liter; $\mathrm{mg} / \mathrm{L}$, milligrams per liter; $\mathrm{mm}$, millimeter. Symbol: --, not determined]

\begin{tabular}{|c|c|c|c|}
\hline \multirow[b]{3}{*}{ Constituent } & \multicolumn{3}{|c|}{ Data-quality objectives } \\
\hline & Detectability & Precision & Bias \\
\hline & $\begin{array}{l}\text { Laboratory reporting } \\
\text { level }\end{array}$ & $\begin{array}{l}\text { Maximum relative standard } \\
\text { deviation of replicate analyses } \\
\text { (percent) }\end{array}$ & $\begin{array}{l}\text { Maximum deviation of spike } \\
\text { recovery (percent) }\end{array}$ \\
\hline Calcium, filtered & $0.02 \mathrm{mg} / \mathrm{L}$ & 20 & -- \\
\hline Arsenic, unfiltered recoverable & $.09 \mu \mathrm{g} / \mathrm{L}$ & 20 & 25 \\
\hline Cadmium, filtered & $.02 \mu \mathrm{g} / \mathrm{L}$ & 20 & 25 \\
\hline Cadmium, unfiltered recoverable & $.05 \mu \mathrm{g} / \mathrm{L}$ & 20 & 25 \\
\hline Copper, filtered & $.50 \mu \mathrm{g} / \mathrm{L}$ & 20 & 25 \\
\hline Lead, filtered & $.01 \mu \mathrm{g} / \mathrm{L}$ & 20 & 25 \\
\hline Lead, unfiltered recoverable & $.04 \mu \mathrm{g} / \mathrm{L}$ & 20 & 25 \\
\hline Manganese, filtered & $.1 \mu \mathrm{g} / \mathrm{L}$ & 20 & 25 \\
\hline Manganese, unfiltered recoverable & $.4 \mu \mathrm{g} / \mathrm{L}$ & 20 & 25 \\
\hline Zinc, filtered & $1.4 \mu \mathrm{g} / \mathrm{L}$ & 20 & 25 \\
\hline Zinc, unfiltered recoverable & $2.4 \mu \mathrm{g} / \mathrm{L}$ & 20 & 25 \\
\hline $\begin{array}{l}\text { Sediment, suspended, percent finer } \\
\text { than } 0.062 \mathrm{~mm}\end{array}$ & 1 percent & 20 & -- \\
\hline Sediment, suspended & $1 \mathrm{mg} / \mathrm{L}$ & 20 & -- \\
\hline
\end{tabular}


because backwater affected the stage-discharge relation. Onsite measurements of $\mathrm{pH}$, specific conductance, and water temperature were made during collection of periodic water samples. Onsite sample processing, including filtration and preservation, was performed according to procedures described by Ward and Harr (1990), Horowitz and others (1994), and the U.S. Geological Survey (variously dated).

Composite water samples were analyzed for the constituents listed in table 2 . The terms "filtered" and "unfiltered recoverable" replace the terms "dissolved" and "total recoverable," respectively, which were used in past reports from this project. Filtered [0.45-micrometer $(\mu \mathrm{m})$ pore size] and unfiltered recoverable concentrations of the trace elements (arsenic, cadmium, copper, iron, lead, manganese, and zinc) and filtered concentrations of calcium and magnesium were measured by the USGS National Water Quality Laboratory (NWQL) in Denver, Colo. Concentrations of calcium and magnesium were used in the calculation of water hardness.

Filtered concentrations of arsenic, cadmium, copper, lead, manganese, and zinc were measured using inductively coupled plasma-mass spectrometry (ICP-MS) (Faires, 1993; Garbarino and others, 2006). Filtered concentrations of calcium, magnesium, and iron were measured using inductively coupled plasma-atomic emission spectrometry (ICP-AES) (Fishman, 1993). Unfiltered recoverable concentrations of trace elements were measured in unfiltered samples that were first digested with dilute hydrochloric acid (Hoffman and others, 1996). For cadmium, iron, lead, and manganese, the digested samples were analyzed by ICP-MS as described by Garbarino and Struzeski (1998). For arsenic, copper, and zinc, the digested samples were analyzed by ICP-MS as described by Garbarino and others (2006).

Water samples for analysis of suspended-sediment also were collected from multiple vertical transits when periodic water samples were collected. These samples were analyzed for suspended-sediment concentration and the percentage of suspended-sediment mass finer than 0.062-millimeter (mm) diameter (silt size and smaller) by the USGS Montana Water Science Center sediment laboratory (hereinafter referred to as the Montana Sediment Laboratory) in Helena, Mont., according to methods described by Guy (1969) and Dodge and Lambing (2006).

Suspended-sediment samples for the four daily suspended-sediment sites (table 1) were collected by local contract observers using the depth-integration method at a single vertical transit near midstream. The samples were analyzed for suspended-sediment concentration and used to calculate daily mean suspended-sediment concentrations according to methods described by Porterfield (1972).

Suspended-sediment discharge is determined according to the following equation (Porterfield, 1972):

$$
Q_{s}=Q_{w} \times C_{s} \times k,
$$

where

$$
\begin{aligned}
& Q_{s} \quad \text { is suspended-sediment discharge, in tons } \\
& \text { per day; } \\
& Q_{w} \quad \text { is streamflow, in cubic feet per second; } \\
& C_{s} \quad \text { is suspended-sediment concentration, in } \\
& \text { milligrams per liter; and } \\
& k \quad \text { is a units-conversion constant (0.0027) } \\
& \text { to convert instantaneous suspended- } \\
& \text { sediment discharge to an equivalent } \\
& \text { daily suspended-sediment discharge. }
\end{aligned}
$$

Turbidity data were measured using continuous turbidity monitors [Yellow Springs Instruments Company (YSI) 6136 turbidity sensor] at four tributary sites in the upper Clark Fork basin near Anaconda (table 1). The monitors were installed in May-June 2006 to provide supporting information on runoff conditions in an area where remediation activities are being conducted. They are operated seasonally, generally from early spring (after ice breakup) to early fall (before stream freeze-up). Turbidity values are recorded at 15-minute intervals and can be viewed in real-time on the Web at $h t t p: / /$ waterdata.usgs.gov/mt/nwis/current?type $=$ quality. Continuous recordings enable the determination of the minimum and maximum values for each day as well as a daily mean turbidity, which is based on the average of all values in a 24-hour period. Procedures for the operation of continuous turbidity monitors and for daily record computations are described by Wagner and others (2006).

\section{Results}

Water-quality data from samples collected periodically during water year 2011 are listed in table 4. Daily mean streamflow, daily mean suspended-sediment concentration, and daily suspended-sediment discharge for water year 2011 at the four daily suspended-sediment sites are listed in tables 5 through 8 along with monthly summary statistics and annual totals for streamflow and suspended-sediment discharge. Daily maximum, minimum, and mean turbidity at four sites are listed in tables 9 through 12 along with monthly summary statistics.

\section{Quality Assurance}

Quality-assurance procedures used for the collection and field processing of water samples are described by Ward and Harr (1990), Horowitz and others (1994), Edwards and Glysson (1999), Lambing (2006), and the U.S. Geological Survey (variously dated). Standard procedures used by the NWQL for internal sample handling and quality assurance are described by Friedman and Erdmann (1982), Jones (1987), and Pritt and Raese (1995). Quality-assurance procedures used by the Montana Sediment Laboratory are described by 
Dodge and Lambing (2006). Standard procedures used for the calibration, measurement, and quality assurance of turbidity monitors are described by Anderson (2005).

The quality of analytical results reported for water samples was evaluated using quality-control samples that were submitted from the field and analyzed concurrently in the laboratory with routine samples. These quality-control samples consisted of replicates, spikes, and blanks that provided quantitative information on the precision and bias of the overall field and laboratory process. Each type of quality-control sample was submitted at a proportion equivalent to about 5 percent of the total number of water samples. Therefore, the total number of quality-control samples represented about 15 percent of the total number of water samples.

In addition to the use of quality-control samples submitted from the field, internal quality-assurance practices are performed systematically by the NWQL to provide quality control of analytical procedures (D.L. Stevenson, U.S. Geological Survey, written commun., 2012). These internal practices include analyses of quality-control samples such as calibration standard samples, standard reference water samples, replicate samples, deionized-water blank samples, or spiked samples at a proportion equivalent to at least 10 percent of the sample load. The NWQL participates in a blind-sample program in which standard reference water samples prepared by the USGS Branch of Quality Systems are routinely inserted into the sample line for each analytical method at a frequency proportional to the sample load (http://bqs.usgs. gov). The laboratory also participates in external evaluation studies and audits with the National Environmental Laboratory Accreditation Program, the U.S. Environmental Protection Agency, Environment Canada, and the USGS Branch of Quality Systems, to assess analytical performance.

Replicate data can be collected in different ways to provide an assessment of precision (reproducibility) of analytical results. Replicate samples are two or more samples considered to be essentially identical in composition. Replicate samples can be collected in the field (field replicate) either by repeating the collection process to obtain two or more independent composite samples or by splitting a single composite sample into two or more subsamples. The individual replicate samples are then analyzed separately. Likewise, a single sample can be analyzed two or more times in the laboratory to obtain a measure of analytical precision (laboratory replicate).

Precision of analytical results for field replicates can be affected by numerous sources of variability within the field and laboratory environments, including sample collection, processing, and analysis. To provide data on overall precision for samples exposed to both field and laboratory sources of variability, replicate stream samples for chemical analysis were obtained in the field by splitting a composite stream sample. Replicate stream samples for suspendedsediment analysis were obtained in the field by collecting two independent cross-sectional samples. Analyses of field replicate samples indicate the reproducibility of environmental data that are affected by the combined potential variability introduced by field and laboratory processes.

Precision of analytical results for laboratory replicates, which exclude field sources of variability, was determined using two independent chemical analyses of aliquots from a single sample selected from the group of samples constituting each analytical run. A separate analysis of the sample was made at the beginning and end of each analytical run to provide information on the reproducibility of laboratory analytical results independent of possible variability caused by field sample collection and processing. Laboratory replicates are not obtainable for suspended-sediment samples because the samples are consumed during the analysis.

Spiked samples are used to evaluate bias, which measures the ability of an analytical method to accurately quantify a known amount of analyte added to a sample. Because some constituents in stream water can potentially interfere with the analysis of a sample for a targeted analyte, it is important to determine whether such effects are causing biased (consistently high or low) results. Deionized-water blank samples and aliquots of stream samples were spiked in the laboratory with known amounts of the same trace elements for which water samples were being analyzed. Analyses of spiked blanks indicate if the spiking procedure and analytical method are within control for a water matrix that is presumably free of chemical interference. Analyses of spiked aliquots of stream samples indicate if the chemical matrix of the stream water interferes with the analytical measurement and whether these interferences could contribute substantial bias to reported trace-element concentrations for stream samples.

Deionized-water blank samples were submitted for every field trip and analyzed to identify the presence and magnitude of contamination that could potentially bias analytical results. The type of blank sample routinely tested was a field blank. Field blanks are aliquots of deionized water that are certified as trace-element free and are processed in the field through the sampling equipment used to collect stream samples. These blanks then are subjected to the same processing (sample splitting, filtration, preservation, transportation, and laboratory handling) as stream samples. Blank samples are analyzed for the same constituents as stream samples in order to identify whether any detectable concentrations exist.

All water samples were handled in accordance with chain-of-custody procedures that provide documentation of sample identity, shipment, receipt, and laboratory handling. All routine and quality-control samples submitted from a sampling episode were stored in a secure area of the NWQL and analyzed as a discrete sample group, independent of other samples submitted to the NWQL. Therefore, the qualitycontrol data apply solely to the analytical results for stream samples reported herein and provide a direct measure of data quality for this monitoring program.

Data-quality objectives (table 3 ) were established for water-quality data as part of the study plan for the expanded long-term monitoring program initiated in 1993. The objectives identify the analytical requirements of 
detectability and serve as a guide for identifying questionable data by establishing acceptable limits for precision and bias of laboratory results. Comparisons of quality-control data to data-quality objectives were used to evaluate whether sampling and analytical procedures produced environmentally representative data in a consistent manner. Data that did not meet the objectives were evaluated for acceptability. If necessary, additional quality-control samples were submitted and corrective action was taken.

The NWQL uses a statistically-based convention for establishing minimum laboratory reporting levels (LRLs) for analytical results and for reporting low-concentration data (Childress and others, 1999). Quality-control data are collected by the NWQL on a continuing basis to determine long-term method detection levels (LT-MDLs) and LRLs. These values are reevaluated each year and, consequently, can change from year to year. The methods used to determine the LRLs are designed to limit the likelihood of a possible occurrence of a false positive or false negative error to 1 percent or less. Accordingly, concentrations are reported as less than the LRL for samples in which the analyte was either not detected or did not pass identification criteria. Analytes that are detected at concentrations between the LT-MDL and the LRL and that pass identification criteria are reported as estimated concentrations. Estimated concentrations are noted with a remark code of "E." These data need to be used with the understanding that their uncertainty is greater than that of data reported without the "E" remark code.

The precision of analytical results for a constituent can be determined by estimating a standard deviation of the differences in concentrations between replicate analyses for several sets of samples. These replicate analyses may consist either of individual analyses of a pair of samples considered to be essentially identical (field replicates) or of multiple analyses of an individual sample (laboratory replicates). The differences in concentration between replicate analyses can be used to estimate a standard deviation according to the following equation (Taylor, 1987):

$$
S=\sqrt{\frac{\sum d^{2}}{2 k}}
$$

where

$S \quad$ is the standard deviation of the difference in concentration between replicate analyses;

$d \quad$ is the difference in concentration between each pair of replicate analyses; and

$k \quad$ is the number of pairs of replicate analyses.

Precision also can be expressed as a relative standard deviation $(R S D)$, in percent, which is computed from the standard deviation and the mean concentration for all the replicate analyses. Expressing precision relative to a mean concentration standardizes the comparison of precision among individual constituents. The $R S D$ is calculated according to the following equation (Taylor, 1987):

$$
R S D=\frac{S}{\bar{x}} \times 100
$$

where

$$
\begin{aligned}
R S D & \text { is the relative standard deviation; } \\
S & \text { is the standard deviation; and } \\
\bar{x} & \text { is the mean concentration for all replicate } \\
& \text { analyses. }
\end{aligned}
$$

Paired analyses of field replicates are listed in table 13. The overall precision for each constituent estimated from analyses of field replicates, which include both field and laboratory sources of variability, is listed in table 14 . The data-quality objective used to indicate acceptable precision of results for field replicates was a maximum $R S D$ of 20 percent (table 3). Precision estimates for the analytical results of field replicates were within the 20-percent $R S D$ limit for all constituents (table 14).

The precision for each constituent estimated from laboratory replicate analyses, which include only laboratory sources of variability, is listed in table 15. Statistics for the precision of analytical results for laboratory replicates are calculated by using unrounded values stored in laboratory data files. The data-quality objective used to indicate acceptable precision of results for laboratory replicates was a maximum $R S D$ of 20 percent (table 3). Precision estimates for the laboratory replicates were within the 20-percent $R S D$ limit for all constituents (table 15). No adjustments were made to analytical data on the basis of replicate analyses precision.

Recovery efficiency for analyses of constituents is determined by comparison of a sample and a spiked aliquot of the same sample. The data-quality objective for acceptable spike recovery of trace elements in water samples was a maximum deviation of 25 percent from a theoretical 100-percent recovery of added constituent (table 3 ). At the laboratory, a spiked deionized-water blank sample and a spiked aliquot of a stream sample were prepared and analyzed along with the original unspiked sample. The differences between the spiked and unspiked sample concentrations were determined and used to compute recovery, in percent, according to equation 4 :

$$
R=\frac{D}{C} \times 100,
$$

where

$R \quad$ is the spike recovery, in percent;

$D \quad$ is the difference between the spiked and unspiked sample concentrations; and

$C$ is the concentration of material used to spike the sample.

If the spike recovery of a trace element was outside a range of 75 to 125 percent, the instrument was recalibrated and the entire sample set and all spiked samples were reanalyzed for that particular trace element until recoveries were improved to the extent possible. Recovery efficiency 
for individual trace elements in laboratory-spiked deionizedwater blank samples and in laboratory-spiked stream samples is listed in tables 16 and 17 , respectively. The mean spike recovery for deionized-water blank samples spiked with trace elements (table 16) ranged from 93.9 to 111 percent. The 95-percent confidence intervals (Taylor, 1987) for the mean spike recovery for each constituent for which deionized-water blank samples were analyzed (table 16) did not exceed a 25-percent deviation from an expected 100-percent recovery, except for filtered iron (89.3-132 percent), which was caused by one sample with a 138 percent recovery. When the data from this one sample is removed from the data set, filtered iron falls within the data-quality objective limit (88.9-119 percent) for the 95-percent confidence interval. The mean spike recovery for spiked stream samples (table 17) ranged from 86.7 to 108 percent. The 95 -percent confidence intervals for the mean spike recovery for each constituent for which stream water samples were analyzed (table 17) did not exceed a 25-percent deviation from an expected 100-percent recovery. No adjustments were made to analytical data on the basis of the mean spike recovery.

High or low bias is indicated if the 95-percent confidence interval does not include 100-percent recovery, thereby indicating a consistent deviation or bias, either high or low. Confidence intervals for percent recovery include 100 percent for all laboratory-spiked deionized-water blank samples (table 16) except for filtered arsenic (102-109 percent), unfiltered arsenic (90.2-99.3 percent), unfiltered cadmium (92.4-99.0 percent), and unfiltered copper (91.6-96.2 percent). Confidence intervals for percent recovery include 100 percent for all laboratory-spiked stream samples (table 17) except for filter arsenic (101-111 percent), unfiltered arsenic (90.5-99.0 percent), unfiltered cadmium (91.5-96.3 percent), unfiltered copper (85.4-91.2 percent), unfiltered lead (94.5-98.4 percent), unfiltered manganese (88.3-94.2 percent), and unfiltered zinc (80.7-92.7). Because the mean spike recoveries for all constituents of laboratory-spiked stream samples met data-quality objectives (less than a 25-percent deviation from 100-percent recovery), no adjustments were made to analytical results for stream samples on the basis of spike recoveries.

Analytical results for field blanks are listed in table 18. A field blank with constituent concentrations equal to or less than the LRL for the analytical method indicates that the entire process of sample collection, field processing, and laboratory analysis is presumably free of contamination. If detectable concentrations of trace elements in field blanks were equal to or greater than twice the LRL, the concentrations were noted during data review. Analytical results from the field blank collected as part of the subsequent sample set were evaluated for evidence of a consistent trend that could indicate systematic contamination. Sporadic, infrequent exceedances of twice the LRL probably represented random contamination or instrument calibration error that was not persistent in the process and was not likely to cause positive bias in a longterm record of analytical results. However, if concentrations for a particular constituent exceeded twice the LRL in field blanks from two consecutive field trips, additional blank samples were collected from individual components of the processing sequence and were submitted for analysis to identify the source of contamination.

Trace-element concentrations in field blanks (table 18) were almost always less than the LRL. Two concentrations of filtered calcium [0.03 and 0.02 milligrams per liter $(\mathrm{mg} / \mathrm{L})$ ] were equal to or exceeded the LRL of $0.02 \mathrm{mg} / \mathrm{L}$; one concentration of filtered cadmium [0.06 micrograms per liter $(\mu \mathrm{g} / \mathrm{L})$ ] exceeded the LRL of $0.02 \mu \mathrm{g} / \mathrm{L}$; one concentration of filtered copper $(0.61 \mu \mathrm{g} / \mathrm{L})$ exceeded the LRL of $0.50 \mu \mathrm{g} / \mathrm{L}$; two concentrations of filtered lead (both $0.02 \mu \mathrm{g} / \mathrm{L}$ ) exceeded the LRL of $0.01 \mu \mathrm{g} / \mathrm{L}$; one concentration of unfiltered lead $(0.05 \mu \mathrm{g} / \mathrm{L})$ exceeded the LRL of $0.04 \mu \mathrm{g} / \mathrm{L}$; two concentrations of filtered manganese (both $0.3 \mu \mathrm{g} / \mathrm{L}$ ) exceeded the LRL of $0.1 \mu \mathrm{g} / \mathrm{L}$; and two concentrations of filtered zinc $(1.9$ and $1.6 \mu \mathrm{g} / \mathrm{L})$ exceeded the LRL of $1.4 \mu \mathrm{g} / \mathrm{L}$. Because no trends were indicated in subsequent sampling trips or follow-up office equipment blanks, no adjustments were made to water-quality sample results on the basis of these detections.

\section{Bed-Sediment Data}

Bed-sediment data for the long-term monitoring program in the Clark Fork basin consist of trace-element concentrations in the fine-grained (less than $0.063 \mathrm{~mm}$ ) fraction of bedsediment samples. Collection of bulk bed sediment (finegrained plus coarse-grained fractions) was discontinued in 2005; therefore, no bulk bed-sediment analytical results or statistical summaries are presented in this report. Bed-sediment samples are collected once annually at 14 sites (fig. 1 and table 1) during low, stable flow conditions at about the same time of year as previous samples (typically August), to facilitate data comparisons among years. Warm Springs Creek at Warm Springs is sampled once every 3 years rather than once annually.

\section{Methods}

Fine-grained bed-sediment samples were collected in August 2011 using protocols described by Axtmann and Luoma (1991). Samples were collected from the surfaces of streambed deposits in areas near the edge of the stream using an acid-washed polypropylene scoop. Whenever possible, samples were collected from both sides of the stream.

Individual samples of bed sediment were collected by scooping material from the surfaces of three to five randomly selected deposits along pools or low-velocity areas. The three to five individual samples were combined to form a single composite sample. This collection process was repeated three times to obtain three composite samples. Each composite sample was wet-sieved onsite through a $0.063-\mathrm{mm}$ polyestermesh sieve using ambient stream water. The fraction of bed sediment in each composite sample that was finer than 0.063 
$\mathrm{mm}$ was collected in an acid-washed 500-milliliter $(\mathrm{mL})$ polyethylene bottle and transported to the laboratory on ice.

Bed-sediment samples were processed and analyzed at the USGS National Research Program Ecology and Contaminants Project laboratory in Menlo Park, Calif. Bedsediment samples were oven-dried at $60^{\circ} \mathrm{C}$ and ground into smaller particle sizes using an acid-washed, ceramic mortar and pestle. Single aliquots of approximately $0.5-0.6$ grams (g) of sediment from each of the three composite bed-sediment samples were digested using a hot, concentrated, nitric acid reflux according to methods described by Luoma and Bryan (1981). Lab replicates were analyzed by taking an aliquot from one of the three sieved replicate samples at each station. After a 2-week digestion period, the aliquots were evaporated to dryness on a hot plate. The dry residue was reconstituted in $10 \mathrm{~mL}$ of $0.6 \mathrm{~N}$ (normal) hydrochloric acid. The reconstituted aliquots were then filtered through a $0.45-\mu \mathrm{m}$ pore-size filter by using a syringe and in-line disposable filter cartridge. The filtrate was diluted to a $1: 10$ ratio with $0.6 \mathrm{~N}$ hydrochloric acid. These final solutions were analyzed for arsenic, cadmium, chromium, copper, iron, lead, manganese, nickel, and zinc by using inductively coupled plasma-atomic emission spectrometry (ICP-AES). The smallest concentration of a constituent that can be reliably reported for analyses of bed sediment is termed the minimum reporting level (MRL).

\section{Results}

Concentrations of trace elements measured in samples of fine-grained bed sediment collected during August 2011 are listed in table 19. Liquid-phase concentrations, in micrograms per milliliter $(\mu \mathrm{g} / \mathrm{mL})$ (which is equivalent to parts per million; ppm), that were analyzed in the reconstituted aliquots of digested bed sediment were converted to solid-phase concentrations, in micrograms per gram $(\mu \mathrm{g} / \mathrm{g})$, by using the following equation:

$$
\mu g / g=\frac{(\mu \mathrm{g} / \mathrm{mL})(\text { volume of digested sample, in } \mathrm{mL})}{(\text { dry weight of sample, in grams })(\text { dilution ratio })}
$$

The reported solid-phase concentrations (table 19) are the means of all analyses for replicate aliquots from each composite bed-sediment sample collected at the site. Because the conversion from liquid-phase to solid-phase concentration is dependent on both the dilution ratio and the dry weight of the sample, MRLs for some trace elements might differ among stations and among years.

\section{Quality Assurance}

The USGS protocols for field collection and processing of bed-sediment samples are designed to prevent contamination from metal sources. Non-metallic sampling and processing equipment (white plastic scoop, funnel-frame apparatus, and 500-mL sample bottles) were acid-washed and rinsed with deionized water prior to the collection of the first sample. Polyester-mesh sieves were washed in laboratory-grade detergent and rinsed with deionized water. All equipment received a final rinse onsite with native stream water. Sampling equipment used at more than one site was rinsed thoroughly between sites with native stream water. Separate sieves were used at each site and, therefore, did not require between-site cleaning. Bed-sediment samples were collected sequentially at sites along an increasing concentration gradient to minimize effects from potential siteto-site carryover contamination.

Quality assurance of analytical results for bed-sediment samples included laboratory instrument calibration with standard solutions and analysis of quality-control samples designed to identify the presence and magnitude of bias (EllenV. Axtmann, U.S. Geological Survey, written commun., 1994). Quality-control samples consisted of standard reference materials (SRMs) and procedural blanks. Sixteen procedural blanks, a set of ten low-concentration SRMs, and a set of ten high-concentration SRMs were analyzed.

Standard reference materials are commercially prepared materials that have certified concentrations of trace elements. Analyses of SRMs are used to indicate the ability of the method to accurately measure a known quantity of a constituent. Multiple analyses of SRMs are made to derive a mean and 95-percent confidence interval for recovery. Recovery efficiency for trace-element analyses of SRMs for bed sediment is listed in table 20. Two SRMs consisting of agricultural soils representing low and high concentrations of trace elements were analyzed to test recovery efficiency for a range of concentrations similar to those occurring in the bed sediment in streams in the upper Clark Fork basin. The digestion process used to analyze bed-sediment samples is not a "total" digestion (does not liberate elements associated with crystalline lattices); therefore, 100 -percent recovery may not be achieved for elements strongly bound to the sediment. The percent recovery of trace elements for SRM analyses that use less than a total digestion is useful to indicate which trace elements display strong sediment-binding characteristics in the SRM and whether analytical recovery is consistent between multiple sets of analyses.

Although data-quality objectives have not been established for bed sediment, percent recoveries for individual trace elements (table 20) illustrate analytical performance. For copper, iron, manganese, nickel, and zinc, mean SRM recoveries for the low concentration standard (SRM 2709a) ranged from 85.7 to 98.1 percent of the certified concentrations. Mean recoveries for cadmium and chromium were 70.7 and 77.8 percent, respectively; and for arsenic and lead 54.0 and 54.4 percent, respectively. The small range of variation (less than 9 percent) in the 95 -percent confidence interval indicates good reproducibility of multiple analyses of SRM 2709a. For arsenic, cadmium, copper, iron, lead, manganese, nickel, and zinc, mean SRM recoveries for the high concentration standard (SRM 2711a) ranged from 82.6 to 
104 percent of the certified concentrations. Mean recovery for chromium is 75.3 percent for SRM 2711a. The small range of variation (less than 10 percent) for the 95-percent confidence interval indicates good reproducibility of multiple analyses of SRM 2711a. No adjustments were made to trace-element concentrations in bed-sediment samples on the basis of recovery efficiencies.

Procedural blanks for bed-sediment samples consisted of the same reagents used for sample digestion and reconstitution. Concentrated nitric acid used for sample digestion was heated and evaporated to dryness. After evaporation, $0.6 \mathrm{~N}$ hydrochloric acid was added to reconstitute the dry residue. Procedural blanks, therefore, represent the same chemical matrix and exposure to analytical materials and handling as the reagents used to digest and reconstitute bed-sediment samples. Analytical results of procedural blanks for bed sediment (table 21) are reported as a liquid-phase concentration, in micrograms per milliliter. A procedural blank was prepared and analyzed concurrently with bedsediment samples for each site. Concentrations of trace elements in all procedural blanks were less than the MRL indicating no contamination bias, so no adjustments to the data were necessary.

\section{Biological Data}

Biological data for the long-term monitoring program in the Clark Fork basin consist of analyses of trace-element concentrations in the whole-body tissue of aquatic benthic insects. Insect samples are collected once annually at the same 14 sites and on the same dates as bed-sediment samples (fig. 1 and table 1), allowing for a direct comparison of biological data with bed-sediment data among the years. Warm Springs Creek at Warm Springs is sampled once every 3 years rather than once annually.

\section{Methods}

Insect samples were collected using protocols described in Hornberger and others (1997). Benthic insects at immature stages were collected with a large nylon-mesh kick net. A single riffle at each site was sampled repeatedly until an adequate number of individual insects were collected to provide sufficient mass for analysis. Targeted taxa for collection were the order Trichoptera (caddisflies) and the order Plecoptera (stoneflies).

Two caddisfly species of the genus Hydropsyche (Hydropsyche cockerelli and Hydropsyche occidentalis) were targeted for collection in this study because of their occurrence at most sites. Hydropsyche species (spp.) that could not be positively identified were categorized as Hydropsyche spp. or Hydropsyche morosa group (in previous reports). When Hydropsyche could not be found, another caddisfly,
Rhyacophila spp., was collected (specifically, at Clark Fork below Lost Creek, near Galen). The caddisfly Arctopsyche grandis and the stoneflies Claassenia sabulosa and Hesperoperla spp. were collected where available to represent additional insect taxa that are commonly distributed in the Clark Fork basin. In addition, specimens from the caddisfly group Brachycentrus spp. were collected in previous years when targeted taxa were not available.

Samples of each taxon were sorted by genus in the field and placed in acid-washed plastic containers. Samples were frozen in a small amount of ambient stream water on dry ice within 30 minutes of collection. Between 1986 and 1998, macroinvertebrate containers were kept on ice to allow the insects to evacuate their gut contents (depurate) for a period of 6 to 8 hours. Excess water was drained and insects were frozen for transport to the laboratory. Since 1999, samples were immediately frozen on dry ice in the field to reduce the possibility of metal loss through intracellular breakdown during depuration. A comparison of immediately frozen to depurated samples showed that although no substantial difference occurred for most metals, concentrations of copper were about 20 percent lower in the depurated samples than in the samples that were immediately frozen. The data were not adjusted for this difference.

Insect samples were processed and analyzed at the USGS National Research Program Ecology and Contaminants Project laboratory in Menlo Park, Calif. Insects were thawed and rinsed with ultrapure deionized water to remove particulate matter and then sorted to their lowest possible taxonomic level. If large numbers of specimens were collected at a site, similar-sized individuals were composited into replicate subsamples. Subsamples were placed in tared scintillation vials and oven-dried at $70^{\circ} \mathrm{C}$. Subsamples were weighed to obtain a final dry weight and digested by reflux using concentrated nitric acid (Cain and others, 1992). After digestion, insect samples were evaporated to dryness on a hot plate. The dry residue was reconstituted in $0.6 \mathrm{~N}$ hydrochloric acid, filtered through a $0.45-\mu \mathrm{m}$ pore-size filter, and analyzed undiluted by ICP-AES for arsenic, cadmium, chromium, copper, iron, lead, manganese, nickel, and zinc. The smallest concentration of a constituent that can be reliably reported for analyses of biota is termed the MRL.

\section{Results}

Concentrations of trace elements in whole-body tissue of aquatic insects collected during August 2011 are listed in table 22. The variability in the number of composite samples among species and among sites reflects differences in insect abundance, with the number of composite samples increasing with the relative abundance of insects. Liquid-phase concentrations, in $\mu \mathrm{g} / \mathrm{mL}$, analyzed in the reconstituted samples were converted to solid-phase concentrations, in $\mu \mathrm{g} / \mathrm{g}$, by using equation 5 . All tissue samples were analyzed undiluted (dilution ratio 1:1). As with MRLs for trace elements 
in bed sediment, MRLs for trace elements in insects may differ among sites as a result of varied sample weights. In general, the smaller the biological-sample weight (primarily a function of insect abundance), the higher the MRL. Therefore, higher MRLs do not necessarily imply a higher trace-element concentration in tissue.

\section{Quality Assurance}

The protocols for field collection and processing of biota samples are designed to prevent contamination from metal sources. Nonmetallic nets, sampling equipment, and processing equipment were used in all sample collection. Equipment was acid-washed and rinsed in ultrapure deionized water prior to the first sample collection. Nets and equipment were thoroughly rinsed in ambient stream water at each main-stem site. New nets were used for all tributary sites. Biota samples were collected sequentially at sites along an increasing concentration gradient to minimize effects from potential siteto-site carryover contamination (Hornberger and others, 1997).

Quality assurance of analytical results for biota samples included laboratory-instrument calibration with standard solutions and analyses of quality-control samples designed to quantify precision and to identify the presence and magnitude of bias. Quality-control samples consisted of 12 replicates of the tissue SRM (lobster hepatopancreas) and 14 procedural blanks (one at each site). Quality-control samples were analyzed in a proportion equivalent to about 20 percent of the total number of biota samples.

Recovery efficiency for trace-element analyses of the SRM for biota is listed in table 23. Data-quality objectives have not been established for analytical recovery in biota, but percent recoveries are shown to illustrate analytical performance. Mean SRM recoveries ranged from 71.6 to 129 percent for all constituents. The small range of variation (less than 7 percent) for the 95-percent confidence interval indicates good reproducibility of multiple analyses of TORT-2 for arsenic, cadmium, copper, iron, manganese, nickel, and zinc. However, chromium and lead had a slightly higher variation (13 percent or less). The mean SRM recovery and small range of variation for the 95-percent confidence interval represent a reasonable range of recoveries. No adjustments were made to trace-element concentrations in biota samples on the basis of recovery efficiencies.

Procedural blanks for biota consisted of undiluted aliquots of the same reagents used to digest and reconstitute tissue of aquatic insects. Analytical results of procedural blanks for biota (table 24) are reported as a liquid-phase concentration, in $\mu \mathrm{g} / \mathrm{mL}$. A procedural blank was prepared and analyzed concurrently with biota samples for each site. Concentrations of trace elements in all procedural blanks were less than the MRL; therefore, no adjustments to the data were necessary.

\section{Statistical Summaries of Data}

Statistical summaries of long-term water-quality, bedsediment, and biological data for the Clark Fork basin are listed in tables 25 through 27 for the period of record at each site since 1985. The summaries include the period of record; number of samples; and maximum, minimum, mean, and median concentrations.

Statistical summaries of water-quality data (table 25) are based on results of cross-section samples collected periodically by the USGS for the long-term monitoring program in the Clark Fork basin during the period of record for each site. The summaries do not include data for supplemental samples collected at selected sites that targeted high-flow conditions or maintenance drawdowns of Milltown Reservoir, which might disproportionately skew the long-term statistics relative to the other sites in the network. Statistical summaries of bed-sediment (table 26) and biological data (table 27) are based on results of samples collected once annually during the indicated years. Because not all sites were sampled for bed sediment and biota every year, the data for some sites do not represent a consecutive annual record. Sampling of bulk bed sediment has been discontinued; therefore, a statistical summary is not presented. Statistical summaries are not presented for discontinued sites.

Statistics for bed-sediment data (table 26) are based on the mean trace-element concentrations determined for each year from the mean of the analyses of composite samples. Therefore, the number of samples for bed sediment represents the number of years that the constituent was analyzed. In contrast, statistics for biological data (table 27) are based on individual analyses for each composite sample collected rather than on a single mean concentration for each year. Also, the number of samples for arsenic for both bed sediment and biota is smaller than the number for other trace elements because sampling for arsenic began in September 2003. In addition, the number of samples analyzed for silver in bed sediment is smaller because analysis for this constituent was discontinued in 2004.

Differences in the number of composited biota samples among species reflect differences in species abundance, both within and between sites and among years. As a result, the statistics for biota describe a wider range of variation in trace-element concentrations than would be evident if results from individual composite samples were averaged. The abundance of aquatic insects at a particular site in a given year limits the biomass of the sample, which in turn may result in varied MRLs. When MRLs vary among years, differences in concentration with time are difficult to determine, especially when a large percentage of the samples have concentrations less than MRLs.

The presence or absence of insect species at a given site can vary among years and may result in different taxa being analyzed in the long-term period of record. Because Hydropsyche insects were not sorted to the species level 
during 1986-89, statistics for stations sampled during those years are based on the results of all Hydropsyche species combined. At some sites, statistics for the Hydropsyche morosa group are based on the combined results for two or more species because these samples could not be clearly identified to the species level, but the individual insects had morosa characteristics.

\section{References Cited}

Anderson, C.W., 2005, Turbidity (version 2.0): U.S. Geological Survey Techniques of Water-Resources Investigations, book 9, chap. A6, section 6.7, 64 p. (Also available at $h t t p: / / p u b s . w a t e r . u s g s . g o v / t w r i 9 A 6 /$.

Andrews, E.D., 1987, Longitudinal dispersion of trace metals in the Clark Fork River, Montana, in Averett, R.C., and McKnight, D.M., eds., Chemical quality of water and the hydrologic cycle: Chelsea, Mich., Lewis Publishers, p. 179-191.

Axtmann, E.V., Cain, D.J., and Luoma, S.N., 1997, Effect of tributary inflows on the distribution of trace metals in fine-grained sediment and benthic insects of the Clark Fork River, Montana: Environmental Science and Technology, v. 31 , no. 3 , p. $750-758$.

Axtmann, E.V., and Luoma, S.N., 1991, Large scale distribution of metal contamination in the fine-grained sediment of the Clark Fork River, Montana: Applied Geochemistry, v. 6, no. 6, p. 75-88.

Cain, D.J., Luoma, S.N., and Axtmann, E.V., 1995, Influence of gut content in immature aquatic insects on assessments of environmental metal contamination: Canadian Journal of Fisheries and Aquatic Sciences, v. 52, no. 12, p. 2736-2746.

Cain, D.J., Luoma, S.N., Carter, J.L., and Ferd, S.V., 1992, Aquatic insects as bioindicators of trace element contamination in cobble-bottom rivers and streams: Canadian Journal of Fisheries and Aquatic Sciences, v. 49, no. 10 , p. 2141-2154.

Childress, C.T., Foreman, W.T., Connor, B.F., and Maloney, T.J., 1999, New reporting procedures based on long-term method detection levels and some considerations for interpretations of water-quality data provided by the U.S. Geological Survey National Water Quality Laboratory: U.S. Geological Survey Open-File Report 99-193, 19 p.

Davis, B.E., 2005, A guide to the proper selection and use of federally approved sediment and water-quality samplers: U.S. Geological Survey Open-File Report 2005-1087, 20 p. (Also available at http://pubs.usgs.gov/of/2005/1087.)
Dodge, K.A., Hornberger, M.I., and Axtmann, E.V., 1996, Water-quality, bed-sediment, and biological data (October 1994 through September 1995) and statistical summaries of data for streams in the upper Clark Fork basin, Montana: U.S. Geological Survey Open-File Report 96-432, 109 p.

Dodge, K.A., Hornberger, M.I., and Axtmann, E.V., 1997, Water-quality, bed-sediment, and biological data (October 1995 through September 1996) and statistical summaries of data for streams in the upper Clark Fork basin, Montana: U.S. Geological Survey Open-File Report 97-552, 91 p.

Dodge, K.A., Hornberger, M.I., and Axtmann, E.V., 1998, Water-quality, bed-sediment, and biological data (October 1996 through September 1997) and statistical summaries of data for streams in the upper Clark Fork basin, Montana: U.S. Geological Survey Open-File Report 98-407, 102 p.

Dodge, K.A., Hornberger, M.I., and Bouse, R.M., 1999, Water-quality, bed-sediment, and biological data (October 1997 through September 1998) and statistical summaries of data for streams in the upper Clark Fork basin, Montana: U.S. Geological Survey Open-File Report 99-251, 102 p.

Dodge, K.A., Hornberger, M.I., and David, C.P.C., 2000, Water-quality, bed-sediment, and biological data (October 1998 through September 1999) and statistical summaries of data for streams in the upper Clark Fork basin, Montana: U.S. Geological Survey Open-File Report 00-370, 102 p.

Dodge, K.A., Hornberger, M.I., and David, C.P.C., 2001, Water-quality, bed-sediment, and biological data (October 1999 through September 2000) and statistical summaries of data for streams in the upper Clark Fork basin, Montana: U.S. Geological Survey Open-File Report 01-379, 95 p.

Dodge, K.A., Hornberger, M.I., and David, C.P.C., 2002, Water-quality, bed-sediment, and biological data (October 2000 through September 2001) and statistical summaries of data for streams in the upper Clark Fork basin, Montana: U.S. Geological Survey Open-File Report 02-358, 94 p.

Dodge, K.A., Hornberger, M.I., and Dyke, J.L., 2005, Water-quality, bed-sediment, and biological data (October 2003 through September 2004) and statistical summaries of data for streams in the upper Clark Fork basin, Montana: U.S. Geological Survey Open-File Report 2005-1356, 124 p. (Also available at $h t t p: / / p u b s . u s g s . g o v /$ of/2005/1356.)

Dodge, K.A., Hornberger, M.I., and Dyke, J.L., 2006, Water-quality, bed-sediment, and biological data (October 2004 through September 2005) and statistical summaries of data for streams in the upper Clark Fork basin, Montana: U.S. Geological Survey Open-File Report 2006-1266, 109 p. (Also available at http://pubs.usgs.gov/ of/2006/1266.) 
Dodge, K.A., Hornberger, M.I., and Dyke, J.L., 2007, Water-quality, bed-sediment, and biological data (October 2005 through September 2006) and statistical summaries of long-term data for streams in the Clark Fork basin, Montana: U.S. Geological Survey Open-File Report 2007-1301, 124 p. (Also available at http://pubs.usgs.gov/ of/2007/1301.)

Dodge, K.A., Hornberger, M.I., and Dyke, J.L., 2008, Water-quality, bed-sediment, and biological data (October 2006 through September 2007) and statistical summaries of long-term data for streams in the Clark Fork basin, Montana: U.S. Geological Survey Open-File Report 2008-1318, 132 p. (Also available at http://pubs.usgs.gov/ of/2008/1318.)

Dodge, K.A., Hornberger, M.I., and Dyke, J.L., 2009, Water-quality, bed-sediment, and biological data (October 2007 through September 2008) and statistical summaries of long-term data for streams in the Clark Fork basin, Montana: U.S. Geological Survey Open-File Report 2009-1178, 139 p. (Also available at http://pubs.usgs.gov/ of/2009/1178.)

Dodge, K.A., Hornberger, M.I., and Dyke, J.L., 2010, Water-quality, bed-sediment, and biological data (October 2008 through September 2009) and statistical summaries of long-term data for streams in the Clark Fork basin, Montana: U.S. Geological Survey Open-File Report 2010-1267, 137 p. (Also available at http://pubs.usgs.gov/ of/2010/1267.)

Dodge, K.A., Hornberger, M.I., and Dyke, J.L., 2012, Water-quality, bed-sediment, and biological data (October 2009 through September 2010) and statistical summaries of data for streams in the Clark Fork basin, Montana: U.S. Geological Survey Open-File Report 2011-1314, 120 p. (Also available at $h t t p: / / p u b s . u s g s . g o v / o f / 2011 / 1314$. .)

Dodge, K.A., Hornberger, M.I., and Lavigne, I.R., 2003, Water-quality, bed-sediment, and biological data (October 2001 through September 2002) and statistical summaries of data for streams in the upper Clark Fork basin, Montana: U.S. Geological Survey Open-File Report 03-356, 95 p.

Dodge, K.A., Hornberger, M.I., and Lavigne, I.R., 2004, Water-quality, bed-sediment, and biological data (October 2002 through September 2003) and statistical summaries of data for streams in the upper Clark Fork basin, Montana: U.S. Geological Survey Open-File Report 2004-1340, $107 \mathrm{p}$.

Dodge, K.A., and Lambing, J.H., 2006, Quality-assurance plan for the analysis of suspended sediment by the U.S. Geological Survey in Montana: U.S. Geological Survey Open-File Report 2006-1242, 25 p. (Also available at http://pubs.usgs.gov/of/2006/1242.)
Edwards, T.K., and Glysson, G.D., 1999, Field methods for measurement of fluvial sediment: U.S. Geological Survey Techniques of Water-Resources Investigations, book 3, chap. C2, 89 p. (Also available at $h t t p: / / p u b s . u s g s . g o v / t w r i /$ twri3-c2\%.)

Faires, L.M., 1993, Methods of analysis by the U.S. Geological Survey National Water Quality LaboratoryDetermination of metals in water by inductively coupled plasma-mass spectrometry: U.S. Geological Survey Open-File Report 92-634, 28 p.

Fishman, M.J., ed., 1993, Methods of analysis by the U.S. Geological Survey National Water Quality LaboratoryDetermination of inorganic and organic constituents in water and fluvial sediments: U.S. Geological Survey Open-File Report 93-125, 217 p.

Friedman, L.C., and Erdmann, D.E., 1982, Quality assurance practices for the chemical and biological analyses of water and fluvial sediments: U.S. Geological Survey Techniques of Water-Resources Investigations, book 5, chap. A6, 181 p. (Also available at http://pubs.usgs.gov/twri/twri5a6/.)

Garbarino, J.R., and Struzeski, T.M., 1998, Methods of analysis by the U.S. Geological Survey National Water Quality Laboratory-Determination of elements in wholewater digests using inductively coupled plasma-optical emission spectrometry and inductively coupled plasma-mass spectrometry: U.S. Geological Survey Open-File Report 98-165, 101 p.

Garbarino, J.R., Kanagy, L.K., and Cree, M.E., 2006, Determination of elements in natural-water, biota, sediment, and soil samples using collision/reaction cell inductively coupled plasma-mass spectrometry: U.S. Geological Survey Techniques and Methods, book 5, sec. B, chap. 1, 88 p.

Guy, H.P., 1969, Laboratory theory and methods for sediment analysis: U.S. Geological Survey Techniques of WaterResources Investigations, book 5, chap. C1, 58 p. (Also available at $h t t p: / / p u b s . u s g s . g o v / t w r i / t w r i 5 c 1 /$.

Helsel, D.R., and Cohn, T.A., 1988, Estimation of descriptive statistics for multiply censored water quality data: Water Resources Research, v. 24, no. 12, p. 1997-2004.

Hoffman, G.L., Fishman, M.J., and Garbarino, J.R., 1996, Methods of analysis by the U.S. Geological Survey National Water Quality Laboratory-In-bottle acid digestion of whole-water samples: U.S. Geological Survey Open-File Report 96-225, $28 \mathrm{p}$.

Hornberger, M.I., Lambing, J.H., Luoma, S.N., and Axtmann, E.V., 1997, Spatial and temporal trends of trace metals in surface water, bed sediment, and biota of the upper Clark Fork basin, Montana, 1985-95: U.S. Geological Survey Open-File Report 97-669, 84 p. 
Horowitz, A.J., Demas, C.R., Fitzgerald, K.K., Miller, T.L., and Rickert, D.A., 1994, U.S. Geological Survey protocol for the collection and processing of surface-water samples for the subsequent determination of inorganic constituents in filtered water: U.S. Geological Survey Open-File Report 94-539, $57 \mathrm{p}$.

Jones, B.E., 1987, Quality control manual of the U.S. Geological Survey's National Water Quality Laboratory: U.S. Geological Survey Open-File Report 87-457, 17 p.

Lambing, J.H., 1987, Water-quality data for the Clark Fork and selected tributaries from Deer Lodge to Milltown, Montana, March 1985 through June 1986: U.S. Geological Survey Open-File Report 87-110, 48 p.

Lambing, J.H., 1988, Water-quality data (July 1986 through September 1987) and statistical summaries (March 1985 through September 1987) for the Clark Fork and selected tributaries from Deer Lodge to Missoula, Montana: U.S. Geological Survey Open-File Report 88-308, 55 p.

Lambing, J.H., 1989, Water-quality data (October 1987 through September 1988) and statistical summaries (March 1985 through September 1988) for the Clark Fork and selected tributaries from Galen to Missoula, Montana: U.S. Geological Survey Open-File Report 89-229, 51 p.

Lambing, J.H., 1990, Water-quality data (October 1988 through September 1989) and statistical summaries (March 1985 through September 1989) for the Clark Fork and selected tributaries from Galen to Missoula, Montana: U.S. Geological Survey Open-File Report 90-168, 68 p.

Lambing, J.H., 1991, Water-quality and transport characteristics of suspended sediment and trace elements in streamflow of the upper Clark Fork basin from Galen to Missoula, Montana, 1985-90: U.S. Geological Survey Water-Resources Investigations Report 91-4139, 73 p.

Lambing, J.H., comp., 2006, Quality-assurance plan for water-quality activities of the U.S. Geological Survey Montana Water Science Center: U.S. Geological Survey Open-File Report 2006-1275, 39 p. (Available online only at http://pubs.usgs.gov/of/2006/1275/.)

Lambing, J.H., Hornberger, M.I., Axtmann, E.V., and Dodge, K.A., 1995, Water-quality, bed-sediment, and biological data (October 1993 through September 1994) and statistical summaries of data for streams in the upper Clark Fork basin, Montana: U.S. Geological Survey Open-File Report 95-429, 104 p.
Lambing, J.H., Hornberger, M.I., Axtmann, E.V., and Pope, D.A., 1994, Water-quality, bed-sediment, and biological data (October 1992 through September 1993) and statistical summaries of water-quality data (March 1985 through September 1993) for streams in the upper Clark Fork basin, Montana: U.S. Geological Survey Open-File Report 94-375, $85 \mathrm{p}$.

Luoma, S.N., and Bryan, G.W., 1981, A statistical assessment of the form of trace metals in oxidized estuarine sediments employing chemical extractants: Science of the Total Environment, v. 17, no. 17, p. 167-196.

Porterfield, George, 1972, Computation of fluvial-sediment discharge: U.S. Geological Survey Techniques of Water-Resources Investigations, book 3, chap. C3, 66 p. (Also available at $h t t p: / / p u b s . u s g s . g o v / t w r i / t w r i 3-c 3 /$.

Pritt, J.W., and Raese, J.W., eds., 1995, Quality assurance/ quality control manual-National Water Quality Laboratory: U.S. Geological Survey Open-File Report 95-443, $35 \mathrm{p}$.

Rantz, S.E., and others, 1982, Measurement and computation of streamflow: U.S. Geological Survey Water-Supply Paper 2175, v.2, $631 \mathrm{p}$.

Taylor, J.K., 1987, Quality assurance of chemical measurements: Chelsea, Mich., Lewis Publishers, 328 p.

U.S. Environmental Protection Agency, 2004, Milltown Reservoir Sediments Operational Unit of the Milltown Reservoir/Clark Fork River Superfund Site-Record of decision, part 2- Decision Summary: U.S. Environmental Protection Agency, 141 p. (Also available at $h t t p: / / w w w . e p a$. gov/region8/superfund/mt/milltown/mrsrod.html.)

U.S. Geological Survey, variously dated, National field manual for the collection of water-quality data: U.S. Geological Survey Techniques of Water-Resources Investigations, book 9, chaps. A1-A9. (Also available at http://pubs.usgs.gov/ twril.)

Wagner, R.J., Boulger, R.W., Oblinger, C.J., and Smith, B.A., 2006, Guidelines and standard procedures for continuous water-quality monitors - Station operation, record computation, and data reporting: U.S. Geological Survey Techniques and Methods 1-D3, 51 p., 8 attachments. (Also available at $h t t p: / / p u b s . u s g s . g o v / t m / 2006 / t m 1 D 3 /$.

Ward, J.R., and Harr, C.A., eds., 1990, Methods for collection and processing of surface-water and bed-material samples for physical and chemical analyses: U.S. Geological Survey Open-File Report 90-140, 71 p. 
Data 

Table 4. Water-quality data for the Clark Fork basin, Montana, October 2010 through September 2011.

[Abbreviations: $\mathrm{CaCO}_{3}$, calcium carbonate; $\mathrm{ft}^{3} / \mathrm{s}$, cubic feet per second; ${ }^{\circ} \mathrm{C}$, degrees Celsius; E, estimated; lab, laboratory; $\mu \mathrm{g} / \mathrm{L}$, micrograms per liter; $\mu \mathrm{S} / \mathrm{cm}$, microsiemens per centimeter at $25^{\circ} \mathrm{C} ; \mathrm{mg} / \mathrm{L}$, milligrams per liter; $\mathrm{mm}$, millimeters; NTRU, nephelometric turbidity ratio unit; ton/d, tons per day. Symbols: <, less than laboratory reporting level; --, no data]

\begin{tabular}{|c|c|c|c|c|c|c|c|c|}
\hline \multicolumn{9}{|c|}{ 12323230--Blacktail Creek at Harrison Avenue, at Butte } \\
\hline Date & Time & $\begin{array}{c}\text { Streamflow, } \\
\text { instanta- } \\
\text { neous } \\
\left(\mathrm{ft}^{3} / \mathrm{s}\right)\end{array}$ & $\begin{array}{c}\text { pH, onsite } \\
\text { (standard } \\
\text { units) }\end{array}$ & $\begin{array}{c}\text { Specific } \\
\text { conduct- } \\
\text { ance, onsite } \\
(\mu S / \mathrm{cm})\end{array}$ & $\begin{array}{c}\text { Temper- } \\
\text { ature, } \\
\text { water } \\
\left({ }^{\circ} \mathrm{C}\right)\end{array}$ & $\begin{array}{l}\text { Hardness, } \\
\text { filtered } \\
(\mathrm{mg} / \mathrm{L} \text { as } \\
\left.\mathrm{CaCO}_{3}\right)\end{array}$ & $\begin{array}{c}\text { Calcium, } \\
\text { filtered } \\
\text { (mg/L) }\end{array}$ & $\begin{array}{c}\text { Magnesium, } \\
\text { filtered } \\
\text { (mg/L) }\end{array}$ \\
\hline $11 / 15 / 2010$ & 0840 & 16 & 7.6 & 261 & 2.0 & 106 & 28.8 & 8.28 \\
\hline 03/14/2011 & 0920 & 15 & 7.3 & 276 & 1.0 & 104 & 28.0 & 8.39 \\
\hline $05 / 16 / 2011$ & 0915 & 55 & 7.5 & 181 & 7.5 & 75.2 & 21.3 & 5.34 \\
\hline $05 / 31 / 2011$ & 0845 & $\mathrm{E} 80$ & 7.6 & 168 & 5.0 & 66.2 & 18.4 & 4.91 \\
\hline $06 / 13 / 2011$ & 0900 & E150 & 7.5 & 156 & 10.0 & 63.2 & 17.9 & 4.49 \\
\hline $07 / 12 / 2011$ & 0845 & 21 & 7.6 & 240 & 12.5 & 99.9 & 28.5 & 7.00 \\
\hline
\end{tabular}

\begin{tabular}{|c|c|c|c|c|c|c|c|c|}
\hline Date & $\begin{array}{c}\text { Arsenic, } \\
\text { filtered } \\
\text { ( } \mu \mathrm{g} / \mathrm{L})\end{array}$ & $\begin{array}{c}\text { Arsenic, } \\
\text { unfiltered } \\
\text { recoverable } \\
(\mu \mathrm{g} / \mathrm{L})\end{array}$ & $\begin{array}{l}\text { Cadmium, } \\
\text { filtered } \\
\text { ( } \mu \mathrm{g} / \mathrm{L})\end{array}$ & $\begin{array}{l}\text { Cadmium, } \\
\text { unfiltered } \\
\text { recoverable } \\
(\mu \mathrm{g} / \mathrm{L})\end{array}$ & $\begin{array}{c}\text { Copper, } \\
\text { filtered } \\
(\mu \mathrm{g} / \mathrm{L})\end{array}$ & $\begin{array}{c}\text { Copper, } \\
\text { unfiltered } \\
\text { recoverable } \\
(\mu \mathrm{g} / \mathrm{L})\end{array}$ & $\begin{array}{c}\text { Iron, } \\
\text { filtered } \\
\text { ( } \mu \mathrm{g} / \mathrm{L})\end{array}$ & $\begin{array}{c}\text { Iron, } \\
\text { unfiltered } \\
\text { recoverable } \\
(\mu \mathrm{g} / \mathrm{L})\end{array}$ \\
\hline $11 / 15 / 2010$ & 2.4 & 3.3 & 0.04 & $<0.05$ & 2.2 & 4.9 & 171 & 611 \\
\hline 03/14/2011 & 3.6 & 4.9 & .05 & .05 & 4.0 & 4.9 & 294 & 640 \\
\hline $04 / 11 / 2011$ & 3.9 & 5.1 & .02 & $<.05$ & 2.6 & 4.1 & 372 & 793 \\
\hline 05/16/2011 & 6.3 & 8.3 & .03 & .07 & 5.9 & 11.0 & 319 & 1,190 \\
\hline $05 / 31 / 2011$ & 6.6 & 7.9 & .03 & $<.05$ & 5.6 & 8.8 & 219 & 695 \\
\hline 06/13/2011 & 12.3 & 13.7 & .03 & $<.05$ & 7.0 & 11.1 & 296 & 835 \\
\hline 07/12/2011 & 8.9 & 11.9 & .03 & $<.05$ & 2.6 & 4.9 & 528 & 1,310 \\
\hline 08/15/2011 & 5.4 & 6.9 & .04 & $<.05$ & 1.8 & 2.5 & 236 & 713 \\
\hline
\end{tabular}

\begin{tabular}{|c|c|c|c|c|c|c|c|c|c|}
\hline Date & $\begin{array}{c}\text { Lead, } \\
\text { filtered } \\
\text { ( } \mu \mathrm{g} / \mathrm{L} \text { ) }\end{array}$ & $\begin{array}{c}\text { Lead, } \\
\text { unfiltered } \\
\text { recoverable } \\
\text { ( } \mu \mathrm{g} / \mathrm{L})\end{array}$ & $\begin{array}{c}\text { Manganese, } \\
\text { filtered } \\
(\mu \mathrm{g} / \mathrm{L})\end{array}$ & $\begin{array}{l}\text { Manganese, } \\
\text { unfiltered } \\
\text { recoverable } \\
\text { ( } \mu \mathrm{g} / \mathrm{L})\end{array}$ & $\begin{array}{c}\text { Zinc, } \\
\text { filtered } \\
\text { ( } \mu \mathrm{g} / \mathrm{L} \text { ) }\end{array}$ & $\begin{array}{c}\text { Zinc, } \\
\text { unfiltered } \\
\text { recoverable } \\
(\mu \mathrm{g} / \mathrm{L})\end{array}$ & $\begin{array}{l}\text { Sediment, } \\
\text { suspended } \\
\text { (percent finer } \\
\text { than } 0.062 \\
\text { mm) }\end{array}$ & $\begin{array}{l}\text { Sediment, } \\
\text { suspended } \\
\text { (mg/L) }\end{array}$ & $\begin{array}{l}\text { Sediment } \\
\text { discharge, } \\
\text { suspended } \\
\text { (ton/d) }\end{array}$ \\
\hline $11 / 15 / 2010$ & 0.07 & 1.15 & 43.6 & 57.9 & 4.0 & 8.8 & 92 & 10 & 0.43 \\
\hline 03/14/2011 & .16 & .50 & 109 & 119 & 4.9 & 5.5 & 69 & 8 & .32 \\
\hline $05 / 16 / 2011$ & .29 & 1.76 & 35.7 & 69.9 & 2.1 & 6.6 & 66 & 25 & 3.7 \\
\hline 05/31/2011 & .19 & .86 & 21.5 & 38.9 & 3.6 & 5.1 & 70 & 12 & E2.6 \\
\hline 06/13/2011 & .29 & 1.21 & 27.1 & 46.6 & 4.0 & 7.3 & 62 & 17 & E6.9 \\
\hline $07 / 12 / 2011$ & .20 & .95 & 74.8 & 136 & 2.1 & 4.4 & 89 & 11 & .62 \\
\hline 08/15/2011 & .11 & .42 & 58.6 & 74.0 & 1.8 & 2.6 & 90 & 6 & .16 \\
\hline
\end{tabular}


Table 4. Water-quality data for the Clark Fork basin, Montana, October 2010 through September 2011.-Continued

[Abbreviations: $\mathrm{CaCO}_{3}$, calcium carbonate; $\mathrm{ft}^{3} / \mathrm{s}$, cubic feet per second; ${ }^{\circ} \mathrm{C}$, degrees Celsius; E, estimated; lab, laboratory; $\mu \mathrm{g} / \mathrm{L}$, micrograms per liter; $\mu \mathrm{S} / \mathrm{cm}$, microsiemens per centimeter at $25^{\circ} \mathrm{C} ; \mathrm{mg} / \mathrm{L}$, milligrams per liter; mm, millimeters; NTRU, nephelometric turbidity ratio unit; ton/d, tons per day. Symbols: <, less than laboratory reporting level; --, no data]

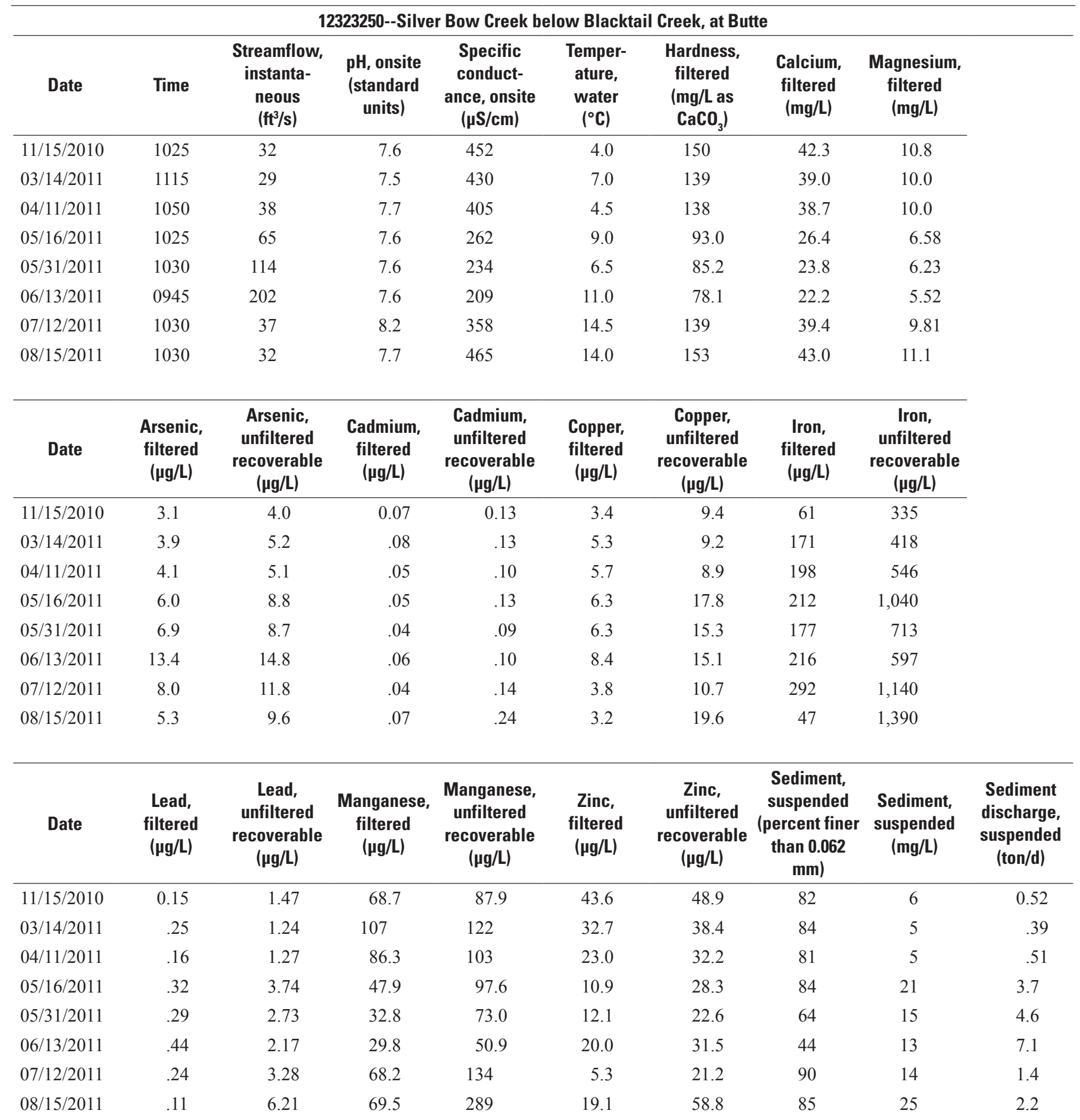


Table 4. Water-quality data for the Clark Fork basin, Montana, October 2010 through September 2011.—Continued

[Abbreviations: $\mathrm{CaCO}_{3}$, calcium carbonate; $\mathrm{ft}^{3} / \mathrm{s}$, cubic feet per second; ${ }^{\circ} \mathrm{C}$, degrees Celsius; E, estimated; lab, laboratory; $\mu \mathrm{g} / \mathrm{L}$, micrograms per liter; $\mu \mathrm{S} / \mathrm{cm}$, microsiemens per centimeter at $25^{\circ} \mathrm{C} ; \mathrm{mg} / \mathrm{L}$, milligrams per liter; $\mathrm{mm}$, millimeters; NTRU, nephelometric turbidity ratio unit; ton/d, tons per day. Symbols: <, less than laboratory reporting level; --, no data]

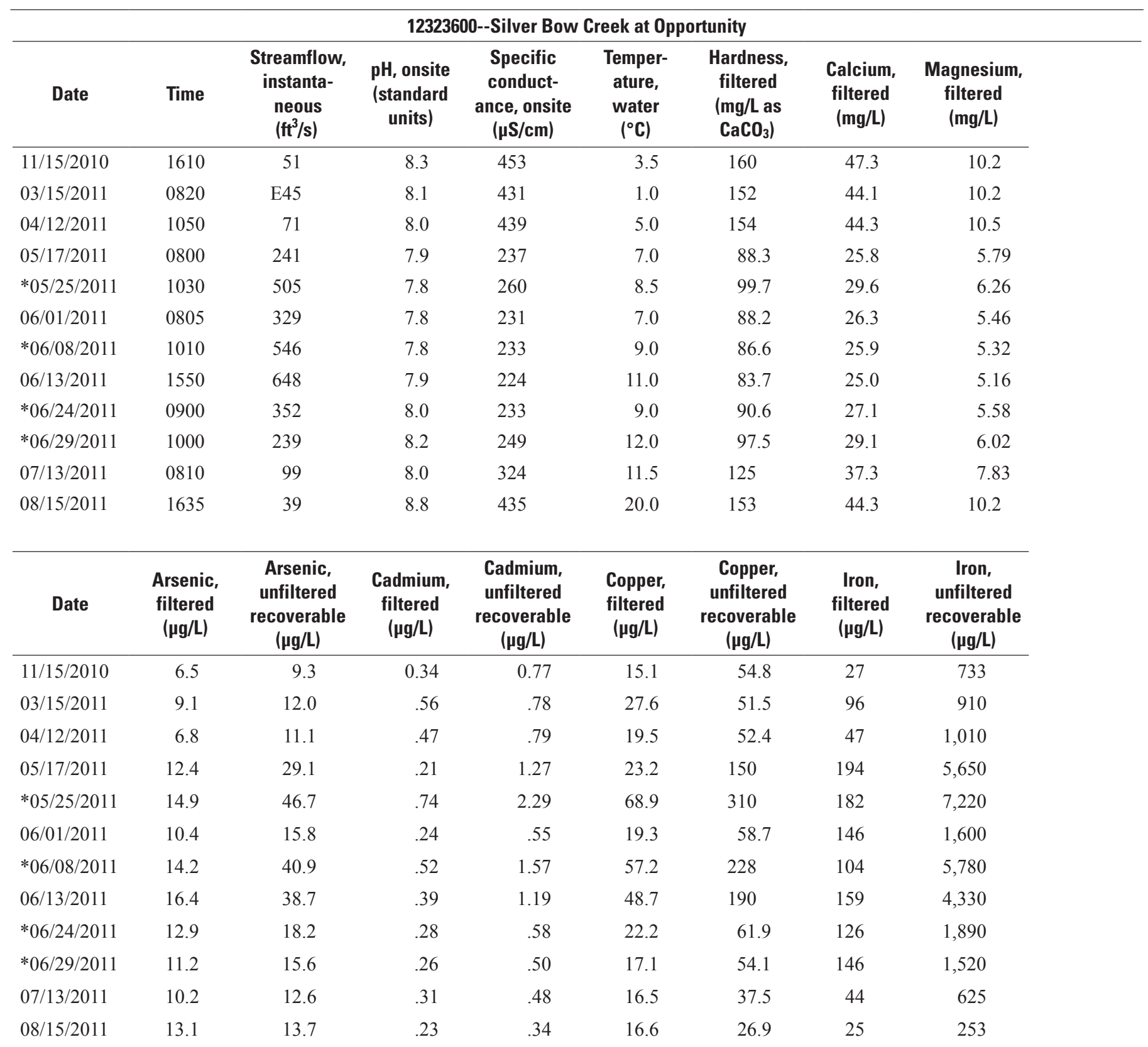




\section{Water-Quality, Bed-Sediment, and Biological Data, and Statistical Summaries of Data, Clark Fork Basin, Montana}

Table 4. Water-quality data for the Clark Fork basin, Montana, October 2010 through September 2011.—Continued

[Abbreviations: $\mathrm{CaCO}_{3}$, calcium carbonate; $\mathrm{ft}^{3} / \mathrm{s}$, cubic feet per second; ${ }^{\circ} \mathrm{C}$, degrees Celsius; E, estimated; lab, laboratory; $\mu \mathrm{g} / \mathrm{L}$, micrograms per liter; $\mu \mathrm{S} / \mathrm{cm}$, microsiemens per centimeter at $25^{\circ} \mathrm{C} ; \mathrm{mg} / \mathrm{L}$, milligrams per liter; mm, millimeters; NTRU, nephelometric turbidity ratio unit; ton/d, tons per day. Symbols: <, less than laboratory reporting level; --, no data]

\begin{tabular}{|c|c|c|c|c|c|c|c|c|c|}
\hline \multicolumn{10}{|c|}{ 12323600--Silver Bow Creek at Opportunity } \\
\hline Date & $\begin{array}{c}\text { Lead, } \\
\text { filtered } \\
\text { ( } \mu \mathrm{g} / \mathrm{L})\end{array}$ & $\begin{array}{c}\text { Lead, } \\
\text { unfiltered } \\
\text { recoverable } \\
(\mu \mathrm{g} / \mathrm{L})\end{array}$ & $\begin{array}{c}\text { Manganese, } \\
\text { filtered } \\
(\mu \mathrm{g} / \mathrm{L})\end{array}$ & $\begin{array}{c}\text { Manganese, } \\
\text { unfiltered } \\
\text { recoverable } \\
(\mu \mathrm{g} / \mathrm{L})\end{array}$ & $\begin{array}{c}\text { Zinc, } \\
\text { filtered } \\
\text { ( } \mu \mathrm{g} / \mathrm{L})\end{array}$ & $\begin{array}{c}\text { Zinc, } \\
\text { unfiltered } \\
\text { recoverable } \\
\text { ( } \mathrm{gg} / \mathrm{L})\end{array}$ & $\begin{array}{c}\text { Sediment, } \\
\text { suspended } \\
\text { (percent finer } \\
\text { than } 0.062 \\
\text { mm) }\end{array}$ & $\begin{array}{c}\text { Sediment, } \\
\text { suspended } \\
(\mathrm{mg} / \mathrm{L})\end{array}$ & $\begin{array}{c}\text { Sediment } \\
\text { discharge, } \\
\text { suspended } \\
\text { (ton/d) }\end{array}$ \\
\hline $11 / 15 / 2010$ & 0.46 & 15.2 & 76.8 & 140 & 90.1 & 156 & 75 & 25 & 3.4 \\
\hline $04 / 12 / 2011$ & .27 & 12.0 & 211 & 310 & 120 & 192 & 65 & 40 & 7.7 \\
\hline $05 / 17 / 2011$ & 1.26 & 48.8 & 73.0 & 404 & 44.4 & 260 & 49 & 206 & 134 \\
\hline$* 05 / 25 / 2011$ & 1.47 & 101 & 158 & 519 & 148 & 463 & 57 & 347 & 473 \\
\hline 06/01/2011 & .73 & 15.7 & 43.4 & 131 & 57.2 & 114 & 50 & 69 & 61 \\
\hline *06/08/2011 & 1.08 & 76.0 & 100 & 358 & 106 & 337 & 56 & 268 & 395 \\
\hline 07/13/2011 & .46 & 8.22 & 83.4 & 124 & 57.0 & 97.8 & 54 & 28 & 7.5 \\
\hline 08/15/2011 & .28 & 3.27 & 45.0 & 69.8 & 19.2 & 52.5 & 90 & 5 & .53 \\
\hline
\end{tabular}


Table 4. Water-quality data for the Clark Fork basin, Montana, October 2010 through September 2011.-Continued

[Abbreviations: $\mathrm{CaCO}_{3}$, calcium carbonate; $\mathrm{ft} / \mathrm{s}$, cubic feet per second; ${ }^{\circ} \mathrm{C}$, degrees Celsius; E, estimated; lab, laboratory; $\mu \mathrm{g} / \mathrm{L}, \mathrm{micrograms}$ per liter; $\mu \mathrm{S} / \mathrm{cm}$, microsiemens per centimeter at $25^{\circ} \mathrm{C} ; \mathrm{mg} / \mathrm{L}$, milligrams per liter; $\mathrm{mm}$, millimeters; NTRU, nephelometric turbidity ratio unit; ton/d, tons per day. Symbols: <, less than laboratory reporting level; --, no data]

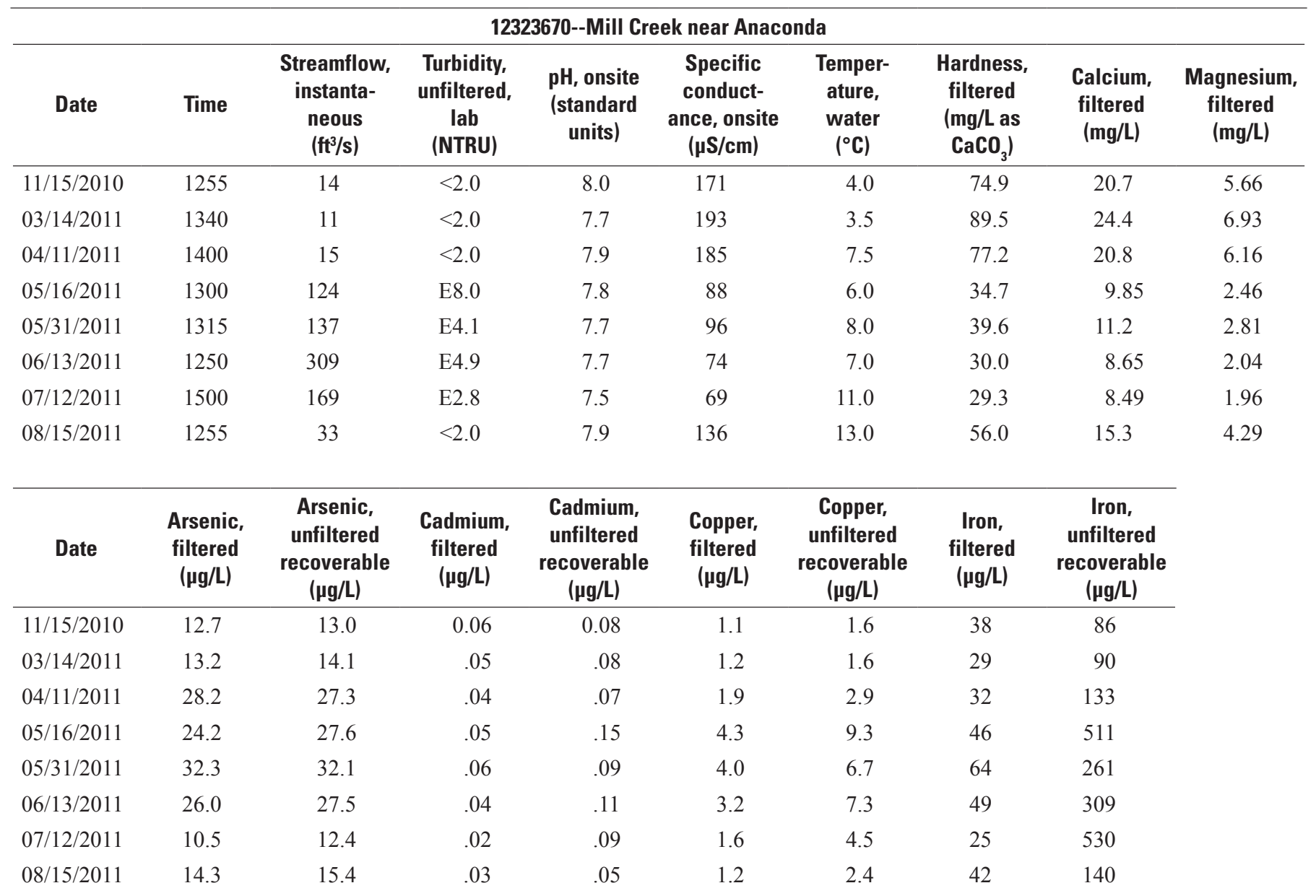

\begin{tabular}{|c|c|c|c|c|c|c|c|c|c|}
\hline Date & $\begin{array}{c}\text { Lead, } \\
\text { filtered } \\
\text { ( } \mu \mathrm{g} / \mathrm{L})\end{array}$ & $\begin{array}{c}\text { Lead, } \\
\text { unfiltered } \\
\text { recoverable } \\
(\mu \mathrm{g} / \mathrm{L})\end{array}$ & $\begin{array}{c}\text { Manganese, } \\
\text { filtered } \\
(\mu \mathrm{g} / \mathrm{L})\end{array}$ & $\begin{array}{l}\text { Manganese, } \\
\text { unfiltered } \\
\text { recoverable } \\
\text { ( } \mu \mathrm{g} / \mathrm{L})\end{array}$ & $\begin{array}{c}\text { Zinc, } \\
\text { filtered } \\
\text { ( } \mu \mathrm{g} / \mathrm{L} \text { ) }\end{array}$ & $\begin{array}{c}\text { Zinc, } \\
\text { unfiltered } \\
\text { recoverable } \\
\text { ( } 1 \mathrm{~g} / \mathrm{L})\end{array}$ & $\begin{array}{c}\text { Sediment, } \\
\text { suspended } \\
\text { (percent finer } \\
\text { than } 0.062 \\
\mathrm{~mm} \text { ) }\end{array}$ & $\begin{array}{l}\text { Sediment, } \\
\text { suspended } \\
\text { (mg/L) }\end{array}$ & $\begin{array}{c}\text { Sediment } \\
\text { discharge, } \\
\text { suspended } \\
\text { (ton/d) }\end{array}$ \\
\hline $11 / 15 / 2010$ & 0.07 & 0.22 & 5.2 & 7.8 & 1.9 & 2.6 & 55 & 1 & 0.04 \\
\hline $03 / 14 / 2011$ & .05 & .28 & 7.0 & 9.1 & 1.8 & $<2.4$ & 80 & 1 & .03 \\
\hline $05 / 16 / 2011$ & .17 & 2.39 & 5.6 & 22.1 & 1.8 & 7.0 & 60 & 21 & 7.0 \\
\hline $05 / 31 / 2011$ & .20 & 1.08 & 4.0 & 10.9 & 2.8 & 4.9 & 56 & 9 & 3.3 \\
\hline 06/13/2011 & .15 & 1.77 & 3.1 & 13.1 & 2.1 & 5.2 & 32 & 15 & 13 \\
\hline $07 / 12 / 2011$ & .07 & 1.23 & 4.4 & 20.4 & $<1.4$ & 6.2 & 35 & 26 & 12 \\
\hline $08 / 15 / 2011$ & .10 & .65 & 9.7 & 15.3 & $<1.4$ & $<2.4$ & 61 & 4 & .36 \\
\hline
\end{tabular}


Table 4. Water-quality data for the Clark Fork basin, Montana, October 2010 through September 2011.—Continued

[Abbreviations: $\mathrm{CaCO}_{3}$, calcium carbonate; $\mathrm{ft}^{3} / \mathrm{s}$, cubic feet per second; ${ }^{\circ} \mathrm{C}$, degrees Celsius; E, estimated; lab, laboratory; $\mu \mathrm{g} / \mathrm{L}$, micrograms per liter; $\mu \mathrm{S} / \mathrm{cm}$, microsiemens per centimeter at $25^{\circ} \mathrm{C} ; \mathrm{mg} / \mathrm{L}$, milligrams per liter; mm, millimeters; NTRU, nephelometric turbidity ratio unit; ton/d, tons per day. Symbols: <, less than laboratory reporting level; --, no data]

\begin{tabular}{|c|c|c|c|c|c|c|c|c|c|}
\hline \multicolumn{10}{|c|}{ 12323700--Mill Creek at Opportunity } \\
\hline Date & Time & $\begin{array}{c}\text { Streamflow, } \\
\text { instanta- } \\
\text { neous } \\
\left(\mathrm{ft}^{3} / \mathrm{s}\right)\end{array}$ & $\begin{array}{c}\text { pH, onsite } \\
\text { (standard } \\
\text { units) }\end{array}$ & $\begin{array}{c}\text { Specific } \\
\text { conduct- } \\
\text { ance, onsite } \\
(\mu \mathrm{S} / \mathrm{cm})\end{array}$ & $\begin{array}{c}\text { Temper- } \\
\text { ature, } \\
\text { water } \\
\left({ }^{\circ} \mathrm{C}\right)\end{array}$ & $\begin{array}{l}\text { Hardness, } \\
\text { filtered } \\
\text { (mg/L as } \\
\mathrm{CaCO}_{3} \text { ) }\end{array}$ & $\begin{array}{c}\text { Calcium, } \\
\text { filtered } \\
\text { (mg/L) }\end{array}$ & $\begin{array}{c}\text { Magnesium, } \\
\text { filtered } \\
\text { (mg/L) }\end{array}$ & \\
\hline $11 / 15 / 2010$ & 1505 & 9.3 & 7.9 & 196 & 3.5 & 85.8 & 24.0 & 6.31 & \\
\hline $03 / 14 / 2011$ & 1600 & 7.1 & 7.8 & 222 & 3.0 & 99.4 & 27.7 & 7.36 & \\
\hline $05 / 16 / 2011$ & 1520 & 87 & 7.7 & 97 & 9.0 & 38.5 & 11.0 & 2.70 & \\
\hline $05 / 31 / 2011$ & 1620 & 113 & 7.9 & 104 & 10.0 & 41.2 & 11.6 & 2.95 & \\
\hline 06/13/2011 & 1425 & 210 & 7.7 & 80 & 7.5 & 32.6 & 9.35 & 2.25 & \\
\hline $07 / 12 / 2011$ & 1855 & 159 & 7.5 & 78 & 11.0 & 32.5 & 9.37 & 2.20 & \\
\hline $11 / 15 / 2010$ & 17.8 & 17.3 & 0.09 & 0.10 & 1.5 & 2.0 & 38 & 74 & \\
\hline $03 / 14 / 2011$ & 17.0 & 18.1 & .06 & .08 & 1.7 & 2.1 & 19 & 59 & \\
\hline $04 / 12 / 2011$ & 21.9 & 24.8 & .06 & .09 & 2.2 & 3.3 & 23 & 107 & \\
\hline $05 / 16 / 2011$ & 29.3 & 36.1 & .07 & .30 & 5.8 & 16.4 & 90 & 930 & \\
\hline $05 / 31 / 2011$ & 33.9 & 35.8 & .07 & .16 & 4.5 & 10.0 & 60 & 477 & \\
\hline $06 / 13 / 2011$ & 30.5 & 34.1 & .06 & .18 & 4.1 & 12.9 & 49 & 1,100 & \\
\hline $07 / 12 / 2011$ & 15.3 & 17.8 & .04 & .12 & 2.3 & 7.2 & 28 & 595 & \\
\hline $11 / 15 / 2010$ & 0.11 & 0.17 & 2.8 & 4.0 & 4.5 & 4.9 & 67 & 1 & 0.03 \\
\hline $03 / 14 / 2011$ & .06 & .20 & 2.4 & 3.2 & 3.1 & 3.2 & 88 & 1 & .02 \\
\hline $04 / 12 / 2011$ & .07 & .39 & 2.1 & 4.9 & 2.9 & 4.4 & 87 & 2 & .06 \\
\hline $05 / 16 / 2011$ & .35 & 5.77 & 4.8 & 39.3 & 3.1 & 14.6 & 53 & 40 & 9.4 \\
\hline $05 / 31 / 2011$ & .22 & 2.16 & 4.1 & 17.4 & 2.8 & 8.1 & 55 & 18 & 5.5 \\
\hline 06/13/2011 & .19 & 3.97 & 2.8 & 26.0 & 3.0 & 12.4 & 31 & 56 & 32 \\
\hline $07 / 12 / 2011$ & .11 & 2.04 & 4.5 & 21.6 & 2.0 & 6.3 & 45 & 38 & 16 \\
\hline $08 / 15 / 2011$ & .11 & .32 & 7.5 & 11.0 & $<1.4$ & $<2.4$ & 80 & 2 & .07 \\
\hline
\end{tabular}


Table 4. Water-quality data for the Clark Fork basin, Montana, October 2010 through September 2011.-Continued

[Abbreviations: $\mathrm{CaCO}_{3}$, calcium carbonate; $\mathrm{ft}^{3} / \mathrm{s}$, cubic feet per second; ${ }^{\circ} \mathrm{C}$, degrees Celsius; E, estimated; lab, laboratory; $\mu \mathrm{g} / \mathrm{L}$, micrograms per liter; $\mu \mathrm{S} / \mathrm{cm}$, microsiemens per centimeter at $25^{\circ} \mathrm{C} ; \mathrm{mg} / \mathrm{L}$, milligrams per liter; $\mathrm{mm}$, millimeters; NTRU, nephelometric turbidity ratio unit; ton/d, tons per day. Symbols: <, less than laboratory reporting level; --, no data]

\begin{tabular}{|c|c|c|c|c|c|c|c|c|c|}
\hline \multicolumn{10}{|c|}{ 12323710--Willow Creek near Anaconda } \\
\hline Date & Time & $\begin{array}{l}\text { Streamflow, } \\
\text { instanta- } \\
\text { neous } \\
\left(\mathrm{ft}^{3} / \mathrm{s}\right)\end{array}$ & $\begin{array}{l}\text { Turbidity, } \\
\text { unfiltered, } \\
\text { lab } \\
\text { (NTRU) }\end{array}$ & $\begin{array}{c}\text { pH, onsite } \\
\text { (standard } \\
\text { units) }\end{array}$ & $\begin{array}{c}\text { Specific } \\
\text { conduct- } \\
\text { ance, onsite } \\
\text { ( } \mathrm{\mu S} / \mathrm{cm})\end{array}$ & $\begin{array}{l}\text { Temper- } \\
\text { ature, } \\
\text { water } \\
\left({ }^{\circ} \mathrm{C}\right)\end{array}$ & $\begin{array}{l}\text { Hardness, } \\
\text { filtered } \\
(\mathrm{mg} / \mathrm{L} \text { as } \\
\left.\mathrm{CaCO}_{3}\right)\end{array}$ & $\begin{array}{c}\text { Calcium, } \\
\text { filtered } \\
\text { (mg/L) }\end{array}$ & $\begin{array}{c}\text { Magnesium, } \\
\text { filtered } \\
\text { (mg/L) }\end{array}$ \\
\hline $11 / 15 / 2010$ & 1200 & 1.6 & $<2.0$ & 7.7 & 133 & 1.5 & 50.4 & 16.5 & 2.21 \\
\hline $04 / 11 / 2011$ & 1245 & 4.0 & $\mathrm{E} 4.0$ & 7.7 & 151 & 5.0 & 56.3 & 18.3 & 2.60 \\
\hline $05 / 31 / 2011$ & 1205 & 78 & E11 & 7.6 & 81 & 5.0 & 30.5 & 10.1 & 1.29 \\
\hline 06/13/2011 & 1150 & 114 & E16 & 7.7 & 72 & 6.0 & 27.0 & 8.91 & 1.14 \\
\hline $07 / 12 / 2011$ & 1355 & 15 & E2.1 & 7.7 & 109 & 12.0 & 42.7 & 14.1 & 1.83 \\
\hline 08/15/2011 & 1200 & 4.1 & E2.1 & 7.7 & 129 & 11.0 & 45.3 & 14.8 & 2.06 \\
\hline $11 / 15 / 2010$ & 11.8 & 11.7 & 0.04 & $<0.05$ & 1.3 & 1.7 & 55 & 119 & \\
\hline $04 / 11 / 2011$ & 16.7 & 16.5 & .02 & .05 & 2.0 & 2.5 & 86 & 307 & \\
\hline $05 / 16 / 2011$ & 23.5 & 26.1 & .04 & .15 & 4.2 & 7.7 & 136 & 1,050 & \\
\hline $05 / 31 / 2011$ & 19.1 & 21.1 & .05 & .12 & 3.5 & 7.4 & 124 & 879 & \\
\hline 06/13/2011 & 19.5 & 23.7 & .04 & .22 & 3.3 & 10.8 & 267 & 2,210 & \\
\hline $07 / 12 / 2011$ & 20.4 & 20.8 & .03 & .05 & 2.4 & 3.4 & 56 & 174 & \\
\hline 08/15/2011 & 23.8 & 23.9 & .03 & .05 & 2.4 & 3.4 & 82 & 203 & \\
\hline $04 / 11 / 2011$ & .25 & .50 & 17.8 & 27.4 & $<1.4$ & $<2.4$ & 93 & 3 & .03 \\
\hline 05/16/2011 & .36 & 2.72 & 9.1 & 42.5 & 2.6 & 8.3 & 64 & 41 & 6.1 \\
\hline $05 / 31 / 2011$ & .26 & 2.45 & 7.6 & 40.2 & 2.7 & 6.8 & 46 & 39 & 8.2 \\
\hline 06/13/2011 & .26 & 5.72 & 8.8 & 72.1 & 2.2 & 14.0 & 30 & 161 & 50 \\
\hline $07 / 12 / 2011$ & .14 & .48 & 24.4 & 30.0 & 1.6 & $<2.4$ & 58 & 8 & .32 \\
\hline 08/15/2011 & .18 & .49 & 28.2 & 34.7 & $<1.4$ & 6.2 & 79 & 5 & .06 \\
\hline
\end{tabular}


Table 4. Water-quality data for the Clark Fork basin, Montana, October 2010 through September 2011.-Continued

[Abbreviations: $\mathrm{CaCO}_{3}$, calcium carbonate; $\mathrm{ft}^{3} / \mathrm{s}$, cubic feet per second; ${ }^{\circ} \mathrm{C}$, degrees Celsius; E, estimated; lab, laboratory; $\mu \mathrm{g} / \mathrm{L}$, micrograms per liter; $\mu \mathrm{S} / \mathrm{cm}$, microsiemens per centimeter at $25^{\circ} \mathrm{C} ; \mathrm{mg} / \mathrm{L}$, milligrams per liter; mm, millimeters; NTRU, nephelometric turbidity ratio unit; ton/d, tons per day. Symbols: <, less than laboratory reporting level; --, no data]

\begin{tabular}{|c|c|c|c|c|c|c|c|c|}
\hline \multicolumn{9}{|c|}{ 12323720--Willow Creek at Opportunity } \\
\hline Date & Time & $\begin{array}{c}\text { Streamflow, } \\
\text { instanta- } \\
\text { neous } \\
\left(\mathrm{ft}^{3} / \mathrm{s}\right)\end{array}$ & $\begin{array}{c}\text { pH, onsite } \\
\text { (standard } \\
\text { units) }\end{array}$ & $\begin{array}{c}\text { Specific } \\
\text { conduct- } \\
\text { ance, onsite } \\
(\mu \mathrm{S} / \mathrm{cm})\end{array}$ & $\begin{array}{l}\text { Temper- } \\
\text { ature, } \\
\text { water } \\
\left({ }^{\circ} \mathrm{C}\right)\end{array}$ & $\begin{array}{l}\text { Hardness, } \\
\text { filtered } \\
\text { (mg/L as } \\
\mathrm{CaCO}_{3} \text { ) }\end{array}$ & $\begin{array}{c}\text { Calcium, } \\
\text { filtered } \\
\text { (mg/L) }\end{array}$ & $\begin{array}{c}\text { Magnesium, } \\
\text { filtered } \\
\text { (mg/L) }\end{array}$ \\
\hline $11 / 15 / 2010$ & 1550 & 8.0 & 8.0 & 302 & 5.5 & 128 & 37.6 & 8.38 \\
\hline $03 / 14 / 2011$ & 1700 & 6.8 & 8.1 & 285 & 5.5 & 124 & 36.3 & 7.99 \\
\hline $05 / 16 / 2011$ & 1550 & 40 & 7.7 & 146 & 11.0 & 58.5 & 18.1 & 3.24 \\
\hline $05 / 31 / 2011$ & 1650 & 62 & 7.7 & 156 & 11.0 & 62.7 & 19.1 & 3.62 \\
\hline $06 / 13 / 2011$ & 1505 & 116 & 7.7 & 167 & 10.0 & 67.8 & 20.5 & 4.03 \\
\hline $07 / 12 / 2011$ & 1930 & 17 & 7.8 & 219 & 14.5 & 91.9 & 27.4 & 5.73 \\
\hline $11 / 15 / 2010$ & 19.3 & 23.0 & 0.03 & 0.06 & 1.8 & 6.1 & 25 & 228 \\
\hline $03 / 14 / 2011$ & 15.9 & 19.2 & .03 & .08 & 2.0 & 5.6 & 46 & 283 \\
\hline $04 / 12 / 2011$ & 16.9 & 21.1 & .03 & .06 & 2.3 & 5.8 & 54 & 284 \\
\hline 05/16/2011 & 38.9 & 49.4 & .04 & .45 & 9.5 & 43.4 & 127 & 1,670 \\
\hline $05 / 31 / 2011$ & 47.5 & 49.2 & .05 & .16 & 9.1 & 21.1 & 111 & 594 \\
\hline 06/13/2011 & 73.7 & 70.6 & .07 & .14 & 11.3 & 20.4 & 100 & 387 \\
\hline
\end{tabular}

\begin{tabular}{|c|c|c|c|c|c|c|c|c|c|}
\hline Date & $\begin{array}{c}\text { Lead, } \\
\text { filtered } \\
\text { ( } \mu \mathrm{g} / \mathrm{L})\end{array}$ & $\begin{array}{c}\text { Lead, } \\
\text { unfiltered } \\
\text { recoverable } \\
(\mu \mathrm{g} / \mathrm{L})\end{array}$ & $\begin{array}{c}\text { Manganese, } \\
\text { filtered } \\
(\mu \mathrm{g} / \mathrm{L})\end{array}$ & $\begin{array}{c}\text { Manganese, } \\
\text { unfiltered } \\
\text { recoverable } \\
(\mu \mathrm{g} / \mathrm{L})\end{array}$ & $\begin{array}{c}\text { Zinc, } \\
\text { filtered } \\
(\mu \mathrm{g} / \mathrm{L})\end{array}$ & $\begin{array}{c}\text { Zinc, } \\
\text { unfiltered } \\
\text { recoverable } \\
(\mu \mathrm{g} / \mathrm{L})\end{array}$ & $\begin{array}{l}\text { Sediment, } \\
\text { suspended } \\
\text { (percent finer } \\
\text { than } 0.062 \\
\text { mm) }\end{array}$ & $\begin{array}{c}\text { Sediment, } \\
\text { suspended } \\
\text { (mg/L) }\end{array}$ & $\begin{array}{r}\text { Sediment } \\
\text { discharge, } \\
\text { suspended } \\
\text { (ton/d) }\end{array}$ \\
\hline $11 / 15 / 2010$ & 0.16 & 1.74 & 25.6 & 33.1 & 2.3 & 9.4 & 81 & 12 & 0.26 \\
\hline 03/14/2011 & .17 & 2.03 & 53.5 & 68.8 & 3.4 & 8.7 & 72 & 9 & .17 \\
\hline $05 / 16 / 2011$ & .61 & 14.4 & 50.3 & 114 & 5.4 & 54.9 & 73 & 87 & 9.4 \\
\hline $05 / 31 / 2011$ & .37 & 4.49 & 15.4 & 37.1 & 8.3 & 23.4 & 54 & 33 & 5.5 \\
\hline $06 / 13 / 2011$ & .33 & 2.88 & 13.6 & 25.7 & 10.2 & 19.1 & 57 & 18 & 5.6 \\
\hline $07 / 12 / 2011$ & .89 & 2.74 & 50.6 & 84.4 & 5.7 & 10.5 & 97 & 13 & .60 \\
\hline $08 / 15 / 2011$ & .11 & .68 & 10.3 & 15.5 & $<1.4$ & 2.9 & 94 & 3 & .05 \\
\hline
\end{tabular}


Table 4. Water-quality data for the Clark Fork basin, Montana, October 2010 through September 2011.-Continued

[Abbreviations: $\mathrm{CaCO}_{3}$, calcium carbonate; $\mathrm{ft}^{3} / \mathrm{s}$, cubic feet per second; ${ }^{\circ} \mathrm{C}$, degrees Celsius; E, estimated; lab, laboratory; $\mu \mathrm{g} / \mathrm{L}$, micrograms per liter; $\mu \mathrm{S} / \mathrm{cm}$, microsiemens per centimeter at $25^{\circ} \mathrm{C}$; mg/L, milligrams per liter; mm, millimeters; NTRU, nephelometric turbidity ratio unit; ton/d, tons per day. Symbols: <, less than laboratory reporting level; --, no data]

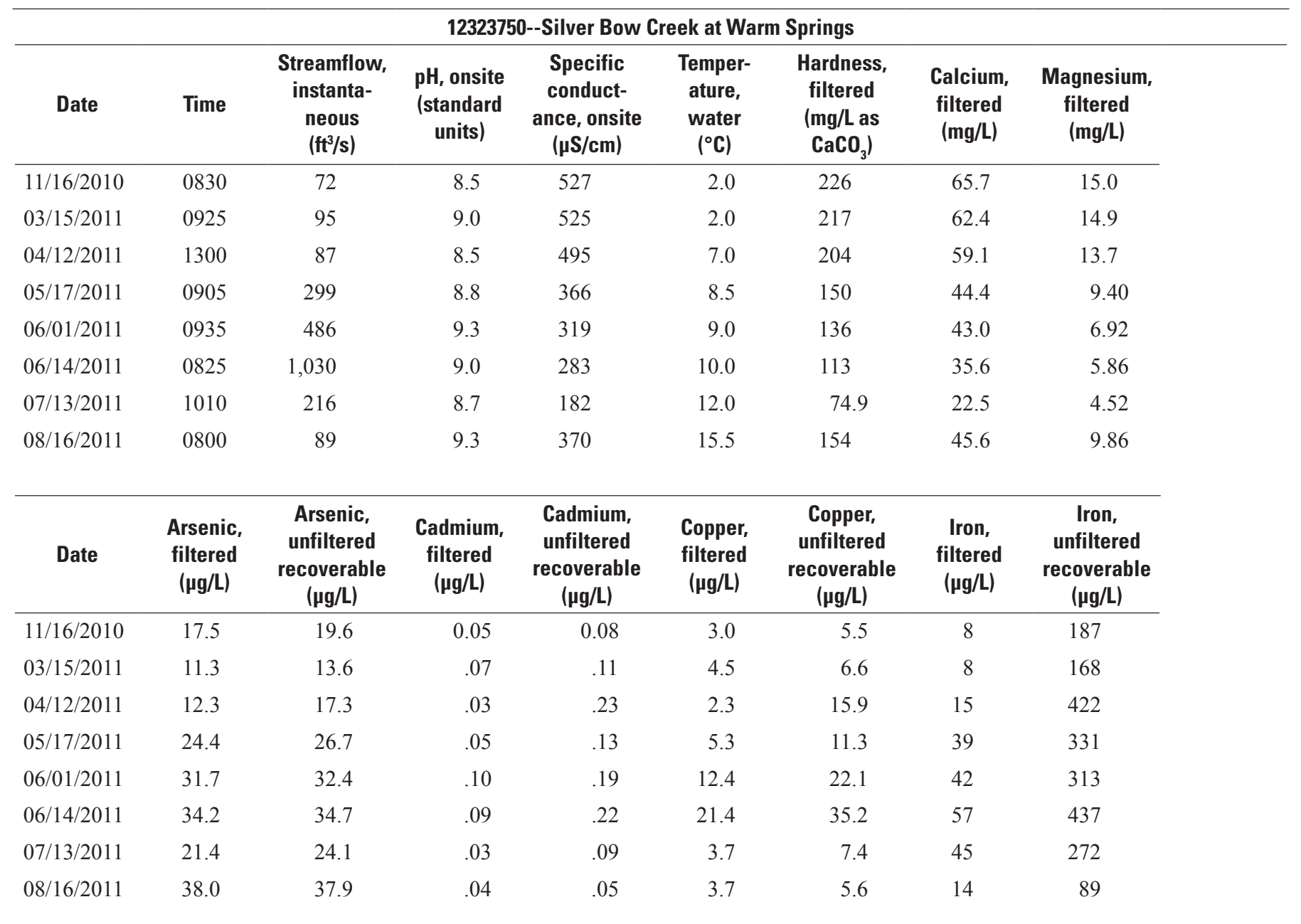

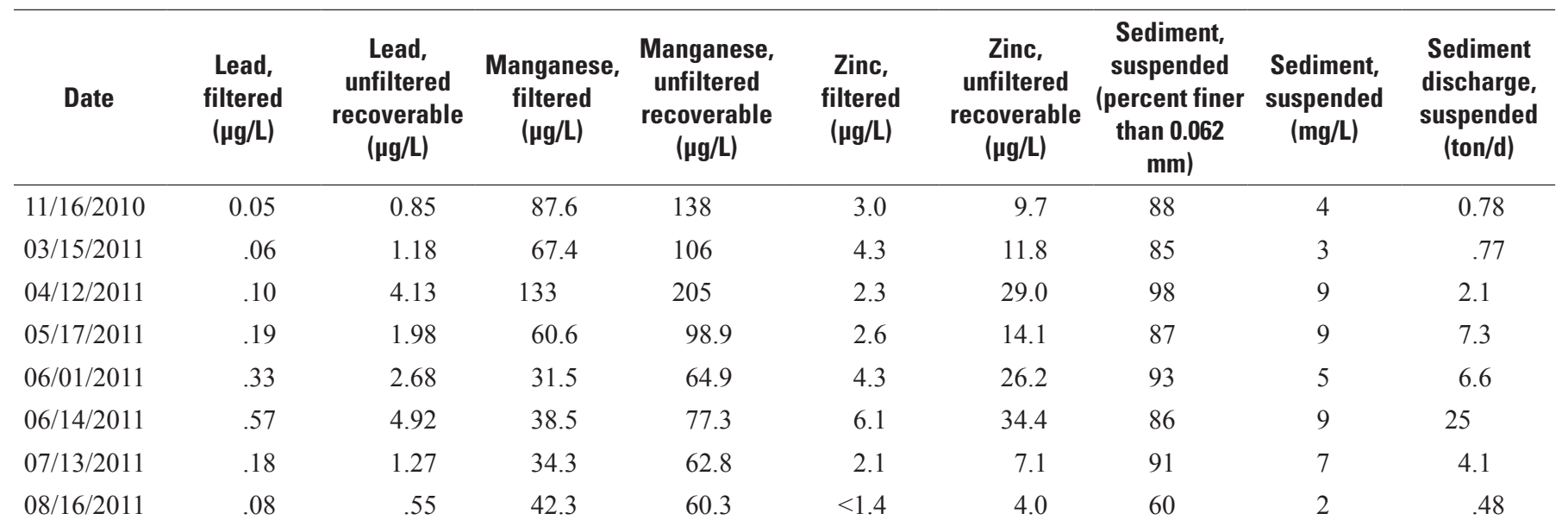


Table 4. Water-quality data for the Clark Fork basin, Montana, October 2010 through September 2011.-Continued

[Abbreviations: $\mathrm{CaCO}_{3}$, calcium carbonate; $\mathrm{ft}^{3} / \mathrm{s}$, cubic feet per second; ${ }^{\circ} \mathrm{C}$, degrees Celsius; E, estimated; lab, laboratory; $\mu \mathrm{g} / \mathrm{L}$, micrograms per liter; $\mu \mathrm{S} / \mathrm{cm}$, microsiemens per centimeter at $25^{\circ} \mathrm{C} ; \mathrm{mg} / \mathrm{L}$, milligrams per liter; mm, millimeters; NTRU, nephelometric turbidity ratio unit; ton/d, tons per day. Symbols: <, less than laboratory reporting level; --, no data]

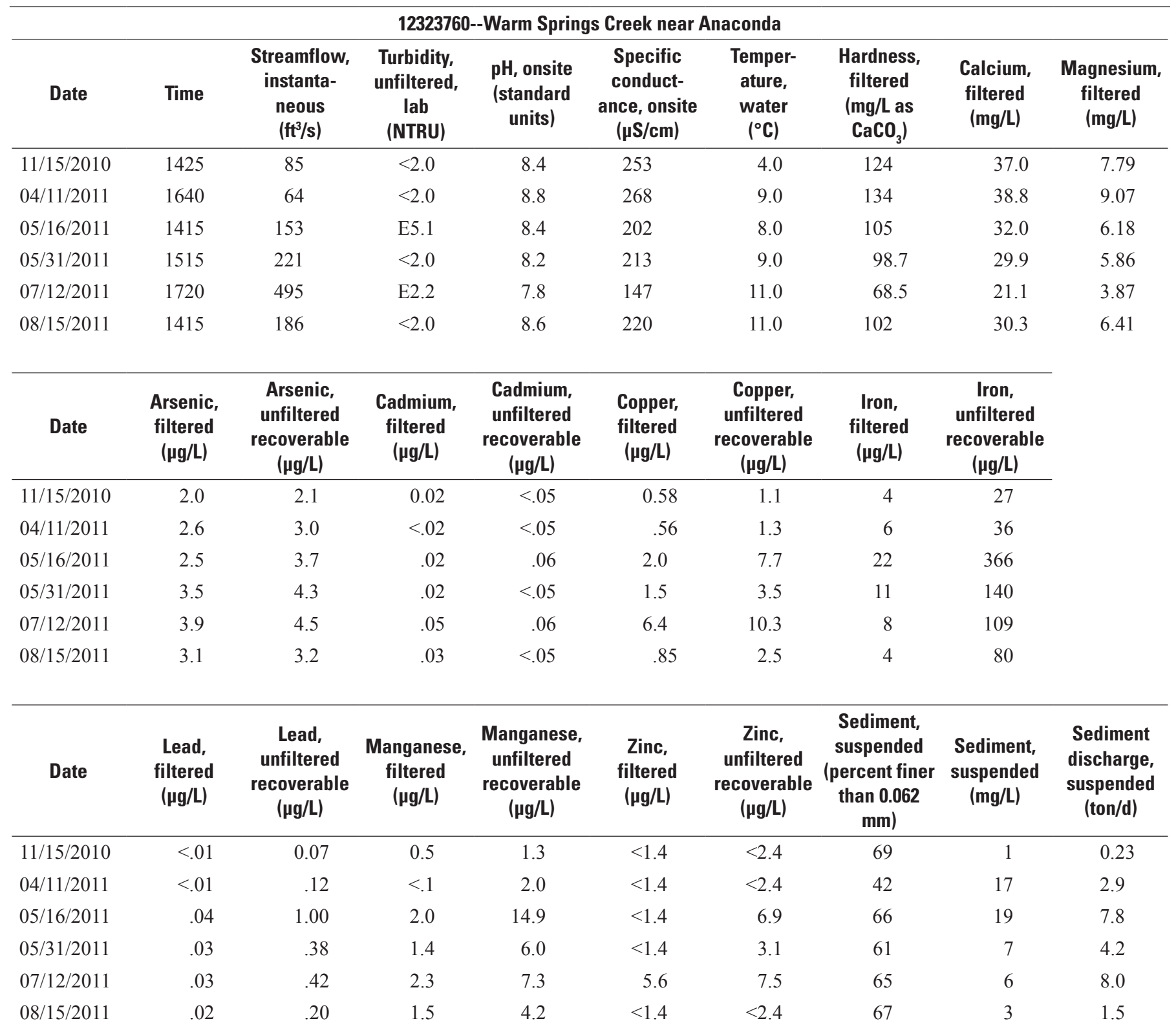


Table 4. Water-quality data for the Clark Fork basin, Montana, October 2010 through September 2011.—Continued

[Abbreviations: $\mathrm{CaCO}_{3}$, calcium carbonate; $\mathrm{ft}^{3} / \mathrm{s}$, cubic feet per second; ${ }^{\circ} \mathrm{C}$, degrees Celsius; E, estimated; lab, laboratory; $\mu \mathrm{g} / \mathrm{L}$, micrograms per liter; $\mu \mathrm{S} / \mathrm{cm}$, microsiemens per centimeter at $25^{\circ} \mathrm{C} ; \mathrm{mg} / \mathrm{L}$, milligrams per liter; $\mathrm{mm}$, millimeters; NTRU, nephelometric turbidity ratio unit; ton/d, tons per day. Symbols: <, less than laboratory reporting level; --, no data]

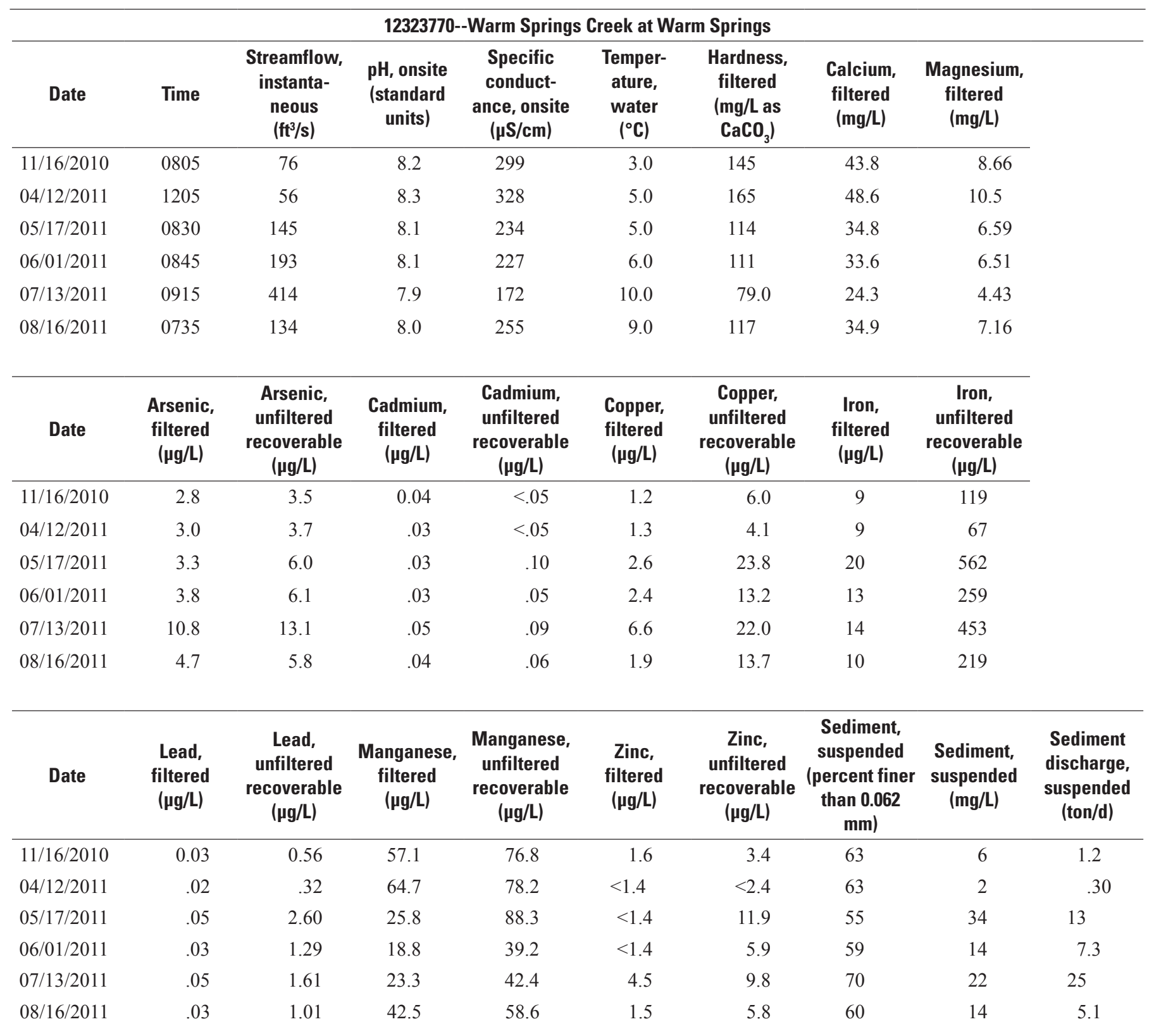


Table 4. Water-quality data for the Clark Fork basin, Montana, October 2010 through September 2011.-Continued

[Abbreviations: $\mathrm{CaCO}_{3}$, calcium carbonate; $\mathrm{ft}^{3} / \mathrm{s}$, cubic feet per second; ${ }^{\circ} \mathrm{C}$, degrees Celsius; E, estimated; lab, laboratory; $\mu \mathrm{g} / \mathrm{L}$, micrograms per liter; $\mu \mathrm{S} / \mathrm{cm}$, microsiemens per centimeter at $25^{\circ} \mathrm{C} ; \mathrm{mg} / \mathrm{L}$, milligrams per liter; mm, millimeters; NTRU, nephelometric turbidity ratio unit; ton/d, tons per day. Symbols: <, less than laboratory reporting level; --, no data]

\begin{tabular}{|c|c|c|c|c|c|c|c|c|}
\hline \multicolumn{9}{|c|}{ 12323800--Clark Fork near Galen } \\
\hline Date & Time & $\begin{array}{c}\text { Streamflow, } \\
\text { instanta- } \\
\text { neous } \\
\left(\mathrm{ft}^{3} / \mathrm{s}\right)\end{array}$ & $\begin{array}{c}\text { pH, onsite } \\
\text { (standard } \\
\text { units) }\end{array}$ & $\begin{array}{c}\text { Specific } \\
\text { conduct- } \\
\text { ance, onsite } \\
(\mu S / \mathrm{cm})\end{array}$ & $\begin{array}{c}\text { Temper- } \\
\text { ature, } \\
\text { water } \\
\left({ }^{\circ} \mathrm{C}\right)\end{array}$ & $\begin{array}{l}\text { Hardness, } \\
\text { filtered } \\
(\mathrm{mg} / \mathrm{L} \text { as } \\
\left.\mathrm{CaCO}_{3}\right)\end{array}$ & $\begin{array}{c}\text { Calcium, } \\
\text { filtered } \\
\text { (mg/L) }\end{array}$ & $\begin{array}{c}\text { Magnesium, } \\
\text { filtered } \\
\text { (mg/L) }\end{array}$ \\
\hline $11 / 16 / 2010$ & 1000 & 162 & 8.3 & 417 & 2.5 & 190 & 56.3 & 12.0 \\
\hline $03 / 15 / 2011$ & 1110 & 172 & 8.7 & 452 & 2.5 & 197 & 57.7 & 13.0 \\
\hline $04 / 12 / 2011$ & 1450 & 155 & 8.6 & 435 & 8.0 & 198 & 58.2 & 12.8 \\
\hline $05 / 17 / 2011$ & 1045 & 445 & 8.6 & 323 & 9.5 & 143 & 42.7 & 8.82 \\
\hline$* 05 / 25 / 2011$ & 1230 & 873 & 8.9 & 300 & 10.5 & 133 & 40.1 & 7.92 \\
\hline 06/01/2011 & 1105 & 664 & 9.1 & 294 & 10.0 & 129 & 40.5 & 6.87 \\
\hline$* 06 / 08 / 2011$ & 1145 & 1,110 & 8.8 & 249 & 9.0 & 106 & 32.6 & 5.83 \\
\hline $06 / 14 / 2011$ & 1020 & 1,380 & 8.7 & 278 & 10.5 & 115 & 36.0 & 6.19 \\
\hline *06/24/2011 & 1030 & 1,210 & 8.9 & 227 & 10.0 & 99.3 & 30.8 & 5.44 \\
\hline *06/29/2011 & 1100 & 1,130 & 8.9 & 228 & 13.5 & 103 & 32.1 & 5.52 \\
\hline 07/13/2011 & 1240 & 628 & 8.2 & 182 & 12.0 & 76.4 & 23.2 & 4.44 \\
\hline 08/16/2011 & 0935 & 215 & 8.7 & 305 & 12.0 & 136 & 40.5 & 8.49 \\
\hline Date & $\begin{array}{c}\text { Arsenic, } \\
\text { filtered } \\
(\mu \mathrm{g} / \mathrm{L})\end{array}$ & $\begin{array}{c}\text { Arsenic, } \\
\text { unfiltered } \\
\text { recoverable } \\
(\mu \mathrm{g} / \mathrm{L})\end{array}$ & $\begin{array}{c}\text { Cadmium, } \\
\text { filtered } \\
\text { ( } \mu \mathrm{g} / \mathrm{L})\end{array}$ & $\begin{array}{c}\text { Cadmium, } \\
\text { unfiltered } \\
\text { recoverable } \\
(\mu \mathrm{g} / \mathrm{L})\end{array}$ & $\begin{array}{c}\text { Copper, } \\
\text { filtered } \\
\text { ( } \mu \mathrm{g} / \mathrm{L})\end{array}$ & $\begin{array}{c}\text { Copper, } \\
\text { unfiltered } \\
\text { recoverable } \\
(\mu \mathrm{g} / \mathrm{L})\end{array}$ & $\begin{array}{c}\text { Iron, } \\
\text { filtered } \\
\text { ( } \mu \mathrm{g} / \mathrm{L})\end{array}$ & $\begin{array}{c}\text { Iron, } \\
\text { unfiltered } \\
\text { recoverable } \\
(\mu \mathrm{g} / \mathrm{L})\end{array}$ \\
\hline $11 / 16 / 2010$ & 9.8 & 11.1 & 0.04 & 0.08 & 2.5 & 6.4 & 10 & 153 \\
\hline $03 / 15 / 2011$ & 8.2 & 10.8 & .05 & .11 & 3.7 & 10.4 & 8 & 210 \\
\hline $04 / 12 / 2011$ & 8.7 & 12.6 & .03 & .16 & 2.5 & 15.3 & 9 & 330 \\
\hline 05/17/2011 & 18.1 & 23.5 & .05 & .23 & 5.1 & 33.6 & 28 & 728 \\
\hline$* 05 / 25 / 2011$ & 29.5 & 39.0 & .08 & .36 & 11.2 & 75.2 & 60 & 1,480 \\
\hline 06/01/2011 & 23.8 & 26.8 & .07 & .20 & 9.7 & 32.0 & 29 & 493 \\
\hline *06/08/2011 & 28.2 & 36.1 & .13 & .38 & 20.7 & 82.1 & 47 & 1,120 \\
\hline $06 / 14 / 2011$ & 27.5 & 31.5 & .11 & .29 & 19.8 & 51.6 & 41 & 629 \\
\hline *06/24/2011 & 26.6 & 27.7 & .10 & .21 & 18.0 & 40.0 & 38 & 576 \\
\hline *06/29/2011 & 24.7 & 25.5 & .09 & .20 & 12.9 & 32.2 & 33 & 461 \\
\hline $07 / 13 / 2011$ & 14.9 & 17.8 & .04 & .10 & 5.9 & 21.9 & 22 & 415 \\
\hline 08/16/2011 & 18.2 & 18.9 & .04 & .07 & 3.6 & 11.2 & 11 & 160 \\
\hline
\end{tabular}


Table 4. Water-quality data for the Clark Fork basin, Montana, October 2010 through September 2011.—Continued

[Abbreviations: $\mathrm{CaCO}_{3}$, calcium carbonate; $\mathrm{ft}^{3} / \mathrm{s}$, cubic feet per second; ${ }^{\circ} \mathrm{C}$, degrees Celsius; E, estimated; lab, laboratory; $\mu \mathrm{g} / \mathrm{L}$, micrograms per liter; $\mu \mathrm{S} / \mathrm{cm}$, microsiemens per centimeter at $25^{\circ} \mathrm{C} ; \mathrm{mg} / \mathrm{L}$, milligrams per liter; $\mathrm{mm}$, millimeters; NTRU, nephelometric turbidity ratio unit; ton/d, tons per day. Symbols: <, less than laboratory reporting level; --, no data]

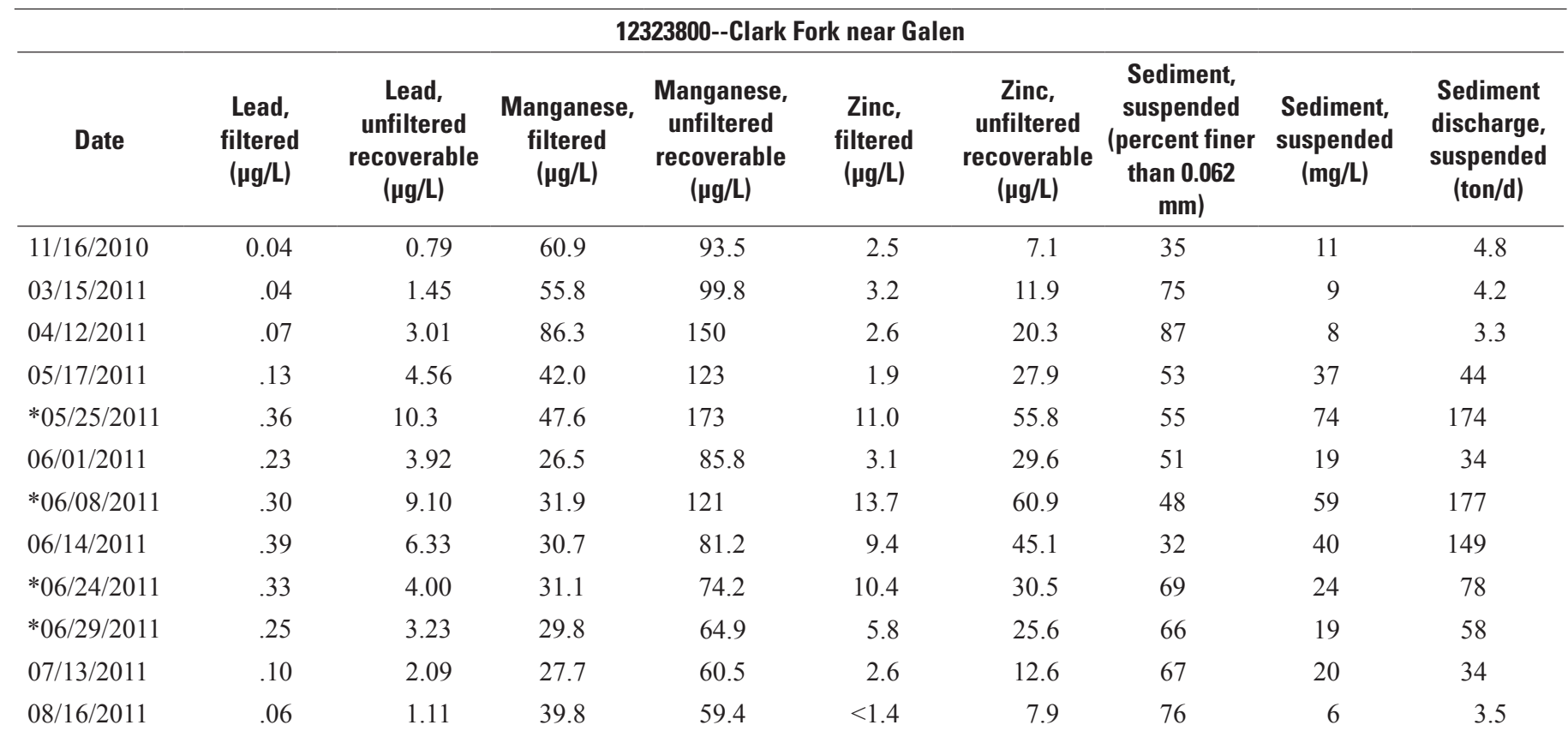


Table 4. Water-quality data for the Clark Fork basin, Montana, October 2010 through September 2011.-Continued

[Abbreviations: $\mathrm{CaCO}_{3}$, calcium carbonate; $\mathrm{ft}^{3} / \mathrm{s}$, cubic feet per second; ${ }^{\circ} \mathrm{C}$, degrees Celsius; E, estimated; lab, laboratory; $\mu \mathrm{g} / \mathrm{L}$, micrograms per liter; $\mu \mathrm{S} / \mathrm{cm}$, microsiemens per centimeter at $25^{\circ} \mathrm{C} ; \mathrm{mg} / \mathrm{L}$, milligrams per liter; mm, millimeters; NTRU, nephelometric turbidity ratio unit; ton/d, tons per day. Symbols: <, less than laboratory reporting level; --, no data]

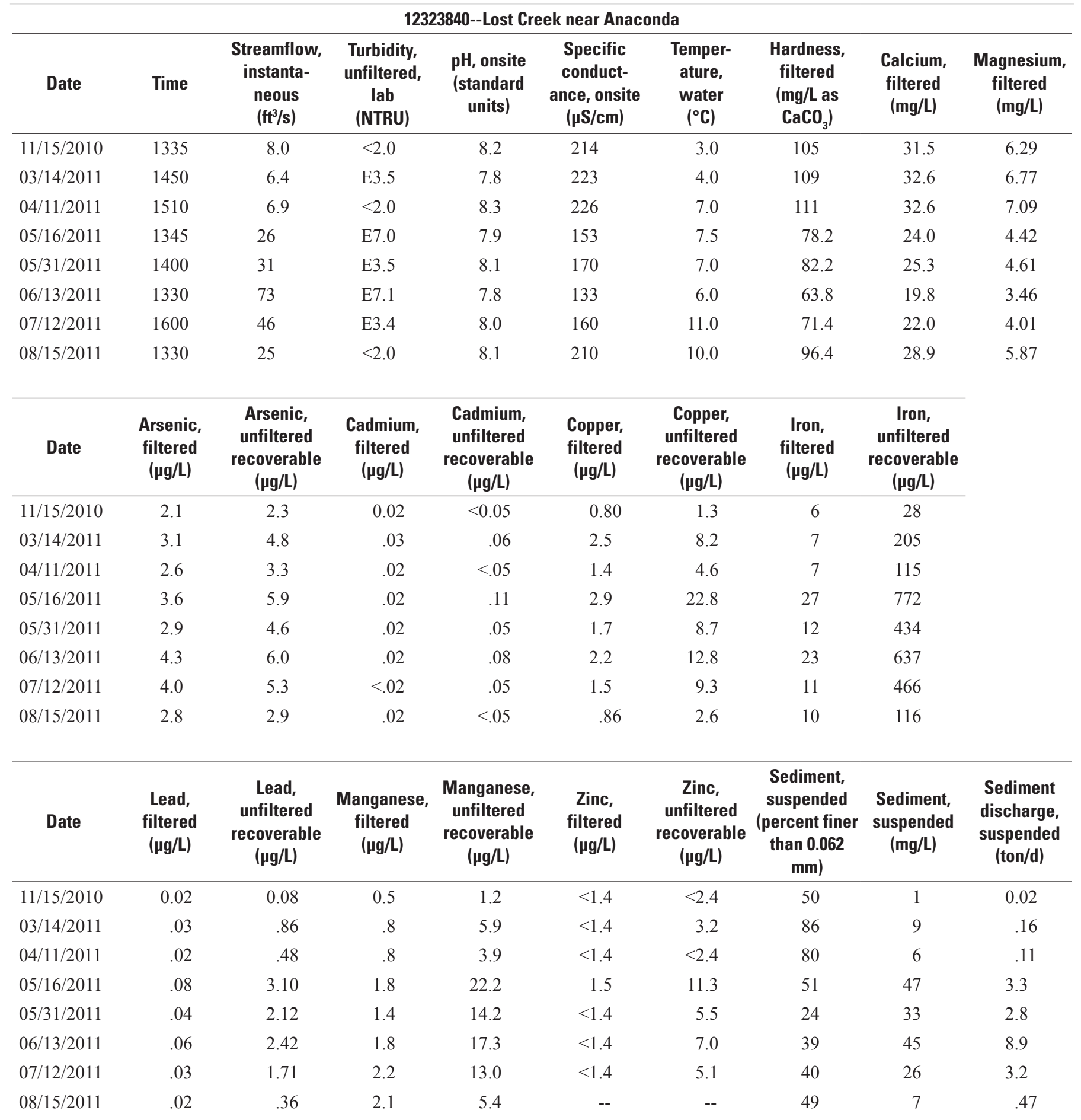


Table 4. Water-quality data for the Clark Fork basin, Montana, October 2010 through September 2011.—Continued

[Abbreviations: $\mathrm{CaCO}_{3}$, calcium carbonate; $\mathrm{ft}^{3} / \mathrm{s}$, cubic feet per second; ${ }^{\circ} \mathrm{C}$, degrees Celsius; E, estimated; lab, laboratory; $\mu \mathrm{g} / \mathrm{L}$, micrograms per liter; $\mu \mathrm{S} / \mathrm{cm}$, microsiemens per centimeter at $25^{\circ} \mathrm{C} ; \mathrm{mg} / \mathrm{L}$, milligrams per liter; $\mathrm{mm}$, millimeters; NTRU, nephelometric turbidity ratio unit; ton/d, tons per day. Symbols: <, less than laboratory reporting level; --, no data]

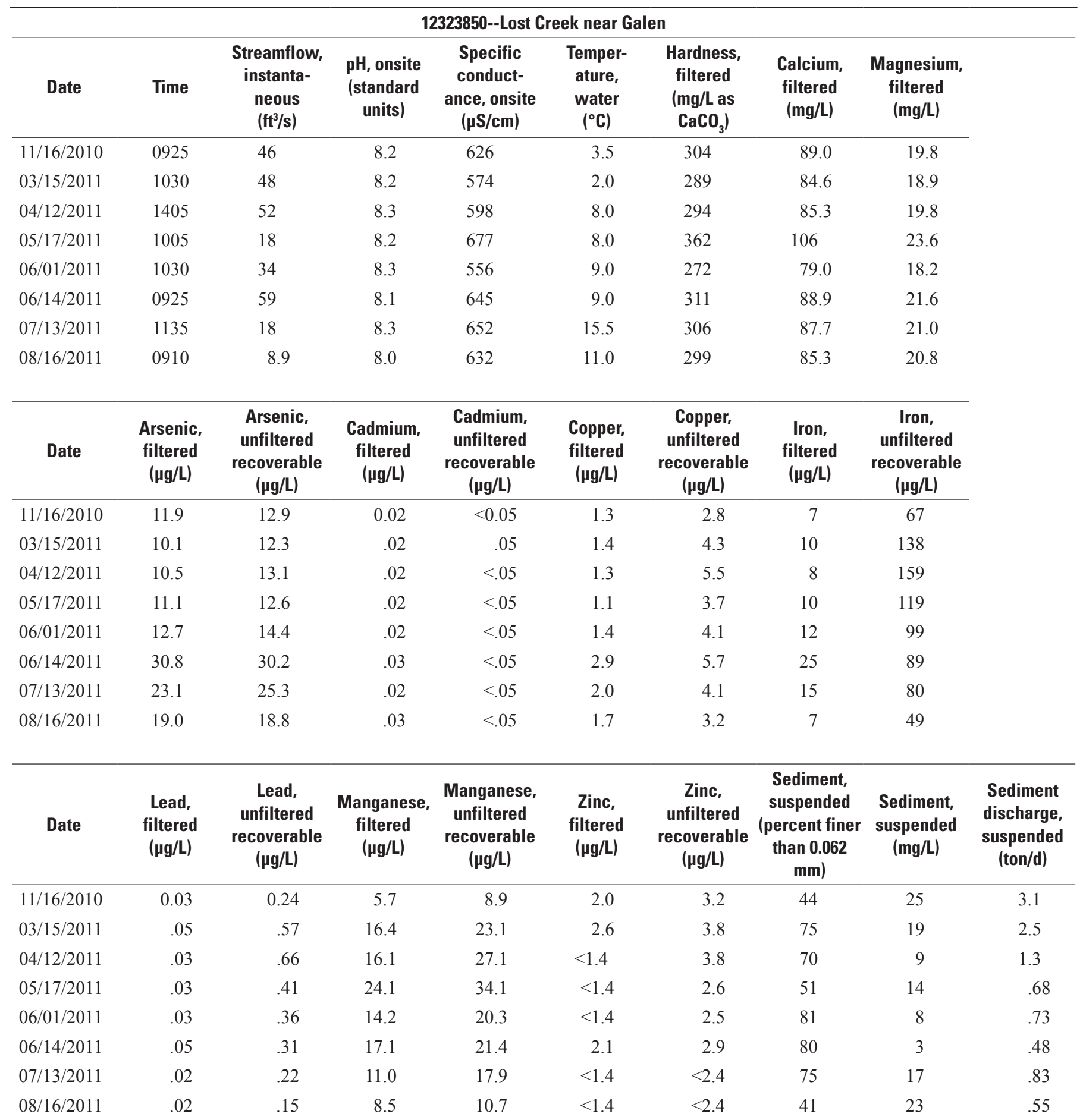


Table 4. Water-quality data for the Clark Fork basin, Montana, October 2010 through September 2011.-Continued

[Abbreviations: $\mathrm{CaCO}_{3}$, calcium carbonate; $\mathrm{ft}^{3} / \mathrm{s}$, cubic feet per second; ${ }^{\circ} \mathrm{C}$, degrees Celsius; E, estimated; lab, laboratory; $\mu \mathrm{g} / \mathrm{L}$, micrograms per liter; $\mu \mathrm{S} / \mathrm{cm}$, microsiemens per centimeter at $25^{\circ} \mathrm{C} ; \mathrm{mg} / \mathrm{L}$, milligrams per liter; mm, millimeters; NTRU, nephelometric turbidity ratio unit; ton/d, tons per day. Symbols: <, less than laboratory reporting level; --, no data]

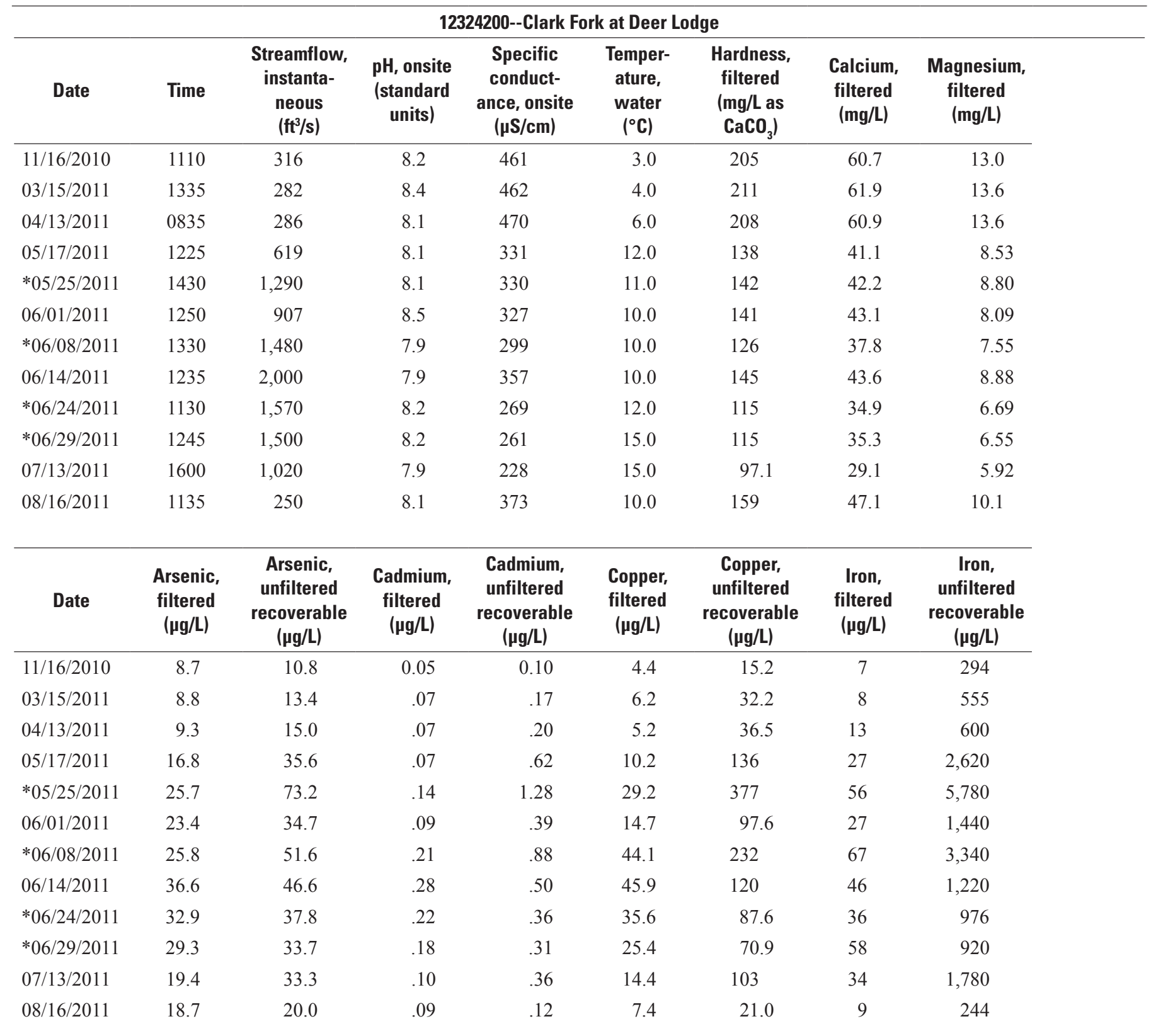


Table 4. Water-quality data for the Clark Fork basin, Montana, October 2010 through September 2011.-Continued

[Abbreviations: $\mathrm{CaCO}_{3}$, calcium carbonate; $\mathrm{ft}^{3} / \mathrm{s}$, cubic feet per second; ${ }^{\circ} \mathrm{C}$, degrees Celsius; E, estimated; lab, laboratory; $\mu \mathrm{g} / \mathrm{L}$, micrograms per liter; $\mu \mathrm{S} / \mathrm{cm}$, microsiemens per centimeter at $25^{\circ} \mathrm{C} ; \mathrm{mg} / \mathrm{L}$, milligrams per liter; $\mathrm{mm}$, millimeters; NTRU, nephelometric turbidity ratio unit; ton/d, tons per day. Symbols: <, less than laboratory reporting level; --, no data]

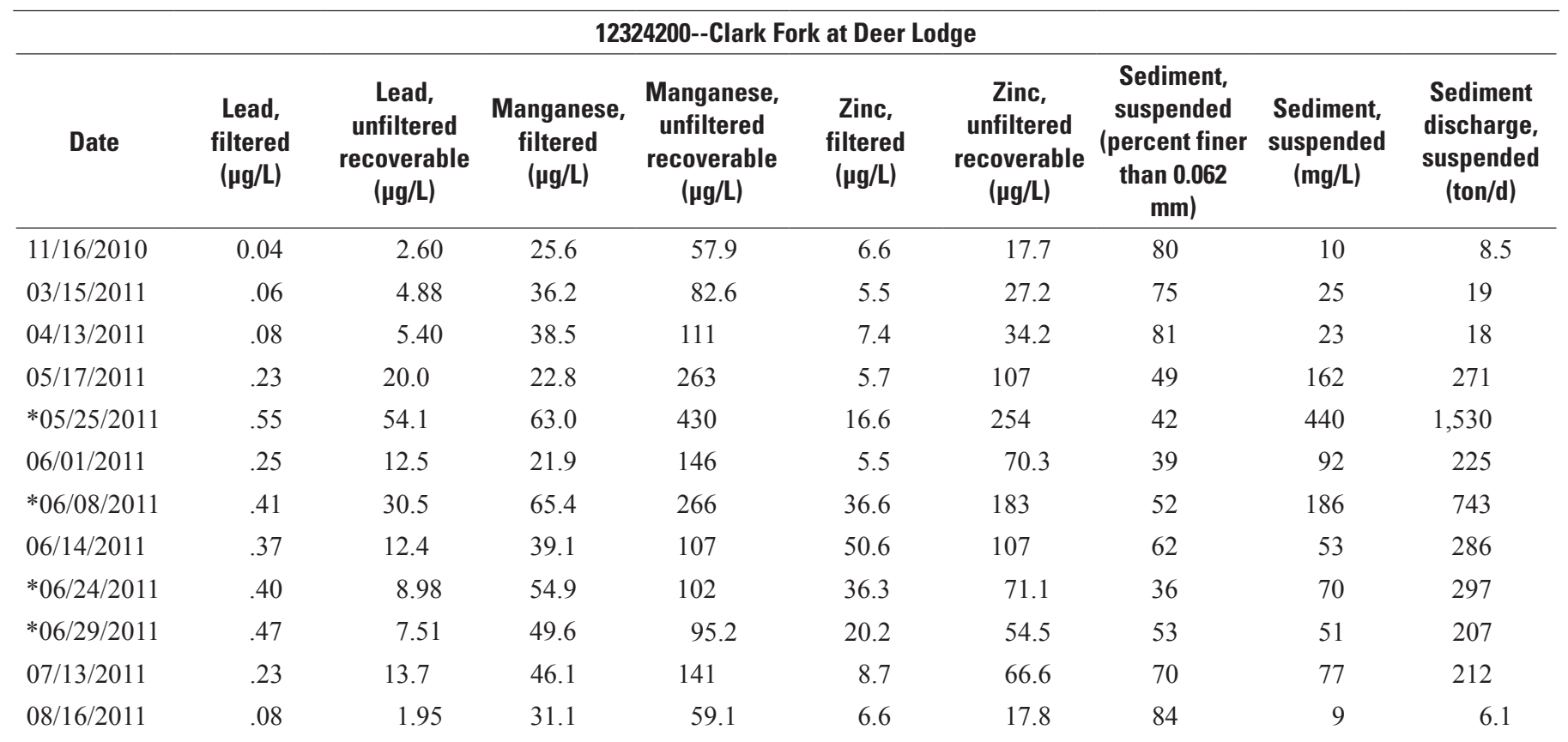


Table 4. Water-quality data for the Clark Fork basin, Montana, October 2010 through September 2011.-Continued

[Abbreviations: $\mathrm{CaCO}_{3}$, calcium carbonate; $\mathrm{ft}^{3} / \mathrm{s}$, cubic feet per second; ${ }^{\circ} \mathrm{C}$, degrees Celsius; E, estimated; lab, laboratory; $\mu \mathrm{g} / \mathrm{L}$, micrograms per liter; $\mu \mathrm{S} / \mathrm{cm}$, microsiemens per centimeter at $25^{\circ} \mathrm{C} ; \mathrm{mg} / \mathrm{L}$, milligrams per liter; mm, millimeters; NTRU, nephelometric turbidity ratio unit; ton/d, tons per day. Symbols: <, less than laboratory reporting level; --, no data]

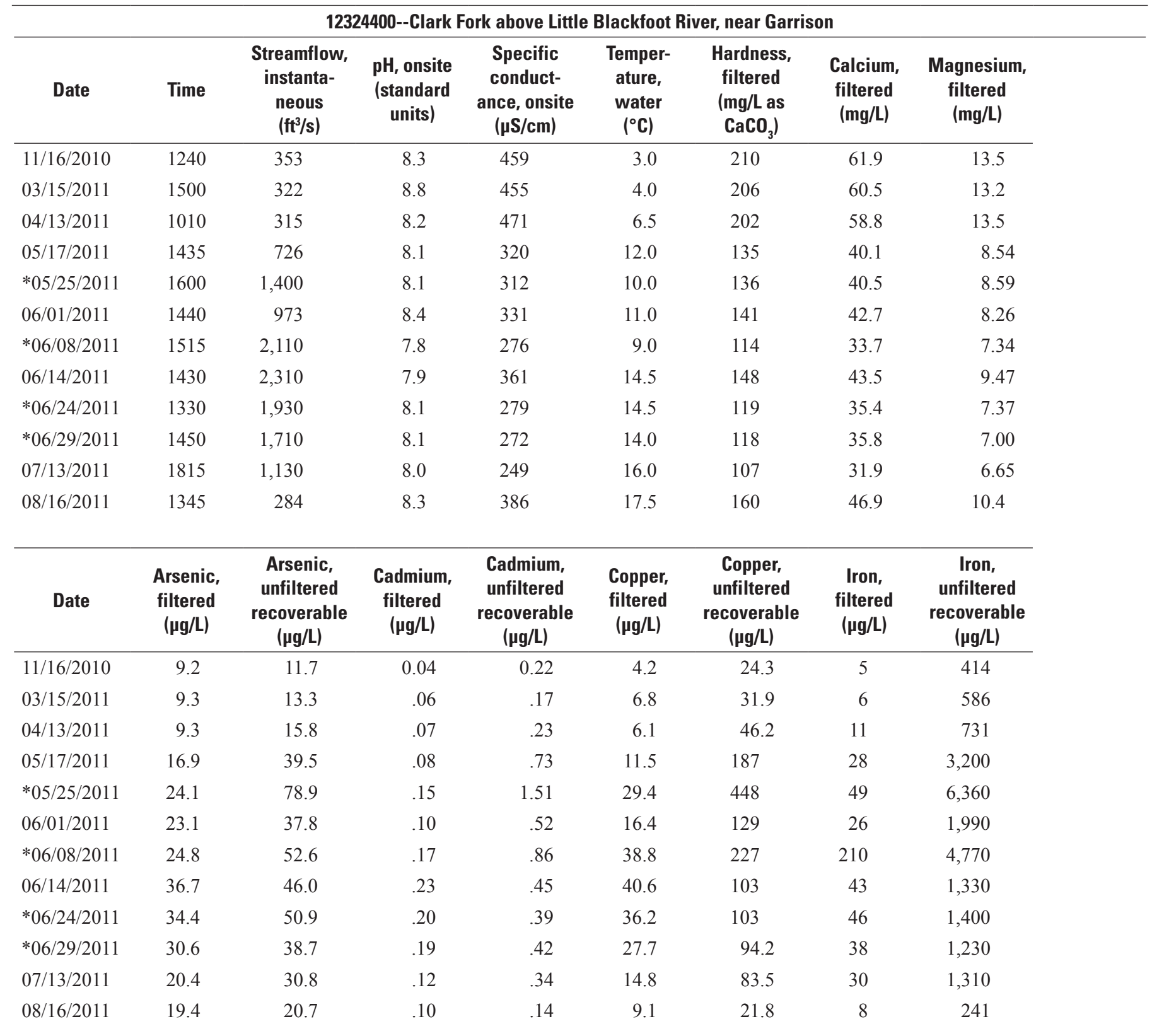


Table 4. Water-quality data for the Clark Fork basin, Montana, October 2010 through September 2011.-Continued

[Abbreviations: $\mathrm{CaCO}_{3}$, calcium carbonate; $\mathrm{ft}^{3} / \mathrm{s}$, cubic feet per second; ${ }^{\circ} \mathrm{C}$, degrees Celsius; E, estimated; lab, laboratory; $\mu \mathrm{g} / \mathrm{L}$, micrograms per liter; $\mu \mathrm{S} / \mathrm{cm}$, microsiemens per centimeter at $25^{\circ} \mathrm{C}$; mg/L, milligrams per liter; mm, millimeters; NTRU, nephelometric turbidity ratio unit; ton/d, tons per day. Symbols: <, less than laboratory reporting level; --, no data]

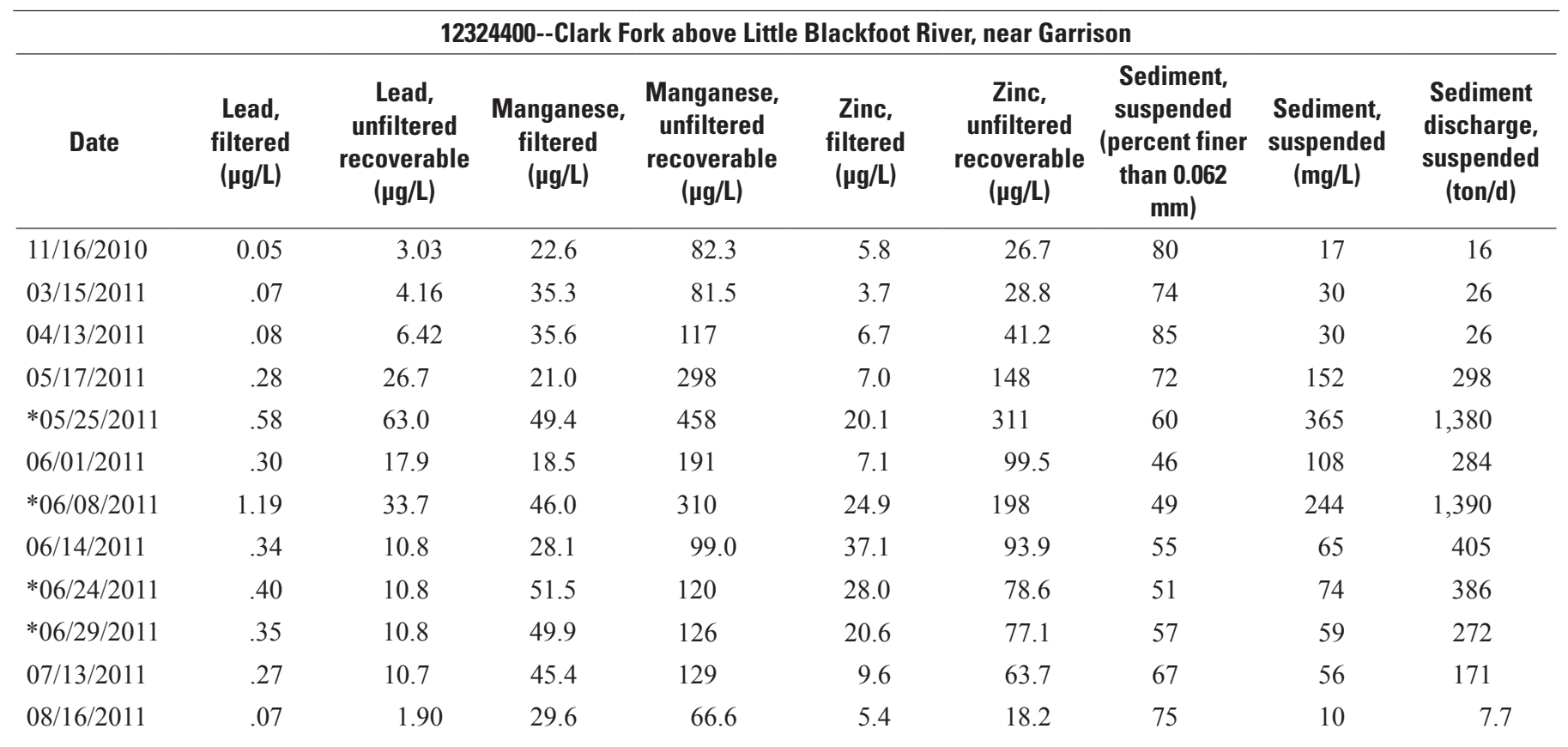


Table 4. Water-quality data for the Clark Fork basin, Montana, October 2010 through September 2011.-Continued

[Abbreviations: $\mathrm{CaCO}_{3}$, calcium carbonate; $\mathrm{ft}^{3} / \mathrm{s}$, cubic feet per second; ${ }^{\circ} \mathrm{C}$, degrees Celsius; E, estimated; lab, laboratory; $\mu \mathrm{g} / \mathrm{L}$, micrograms per liter; $\mu \mathrm{S} / \mathrm{cm}$, microsiemens per centimeter at $25^{\circ} \mathrm{C} ; \mathrm{mg} / \mathrm{L}$, milligrams per liter; mm, millimeters; NTRU, nephelometric turbidity ratio unit; ton/d, tons per day. Symbols: <, less than laboratory reporting level; --, no data]

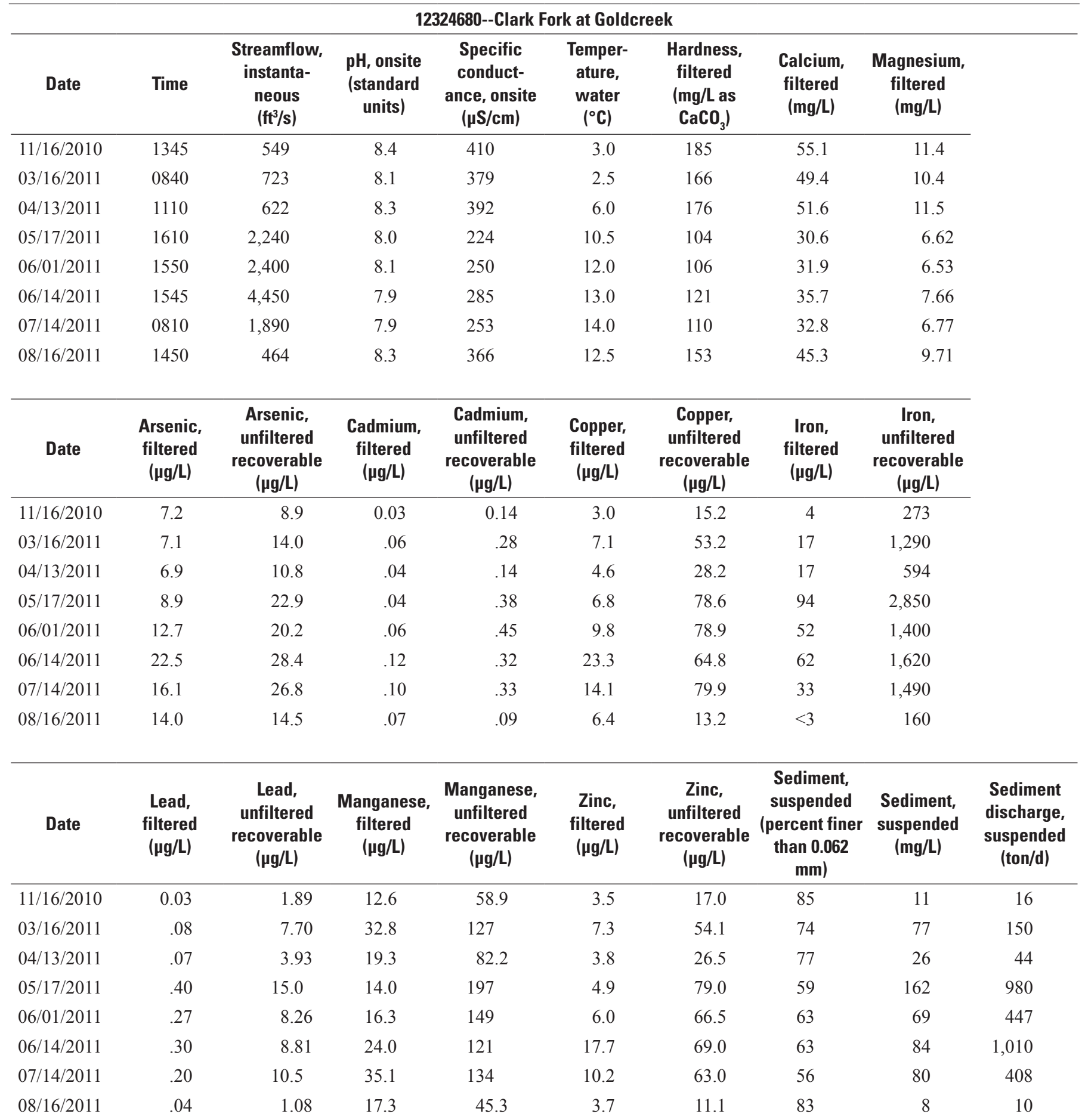


Table 4. Water-quality data for the Clark Fork basin, Montana, October 2010 through September 2011.—Continued

[Abbreviations: $\mathrm{CaCO}_{3}$, calcium carbonate; $\mathrm{ft}^{3} / \mathrm{s}$, cubic feet per second; ${ }^{\circ} \mathrm{C}$, degrees Celsius; E, estimated; lab, laboratory; $\mu \mathrm{g} / \mathrm{L}$, micrograms per liter; $\mu \mathrm{S} / \mathrm{cm}$, microsiemens per centimeter at $25^{\circ} \mathrm{C} ; \mathrm{mg} / \mathrm{L}$, milligrams per liter; $\mathrm{mm}$, millimeters; NTRU, nephelometric turbidity ratio unit; ton/d, tons per day. Symbols: <, less than laboratory reporting level; --, no data]

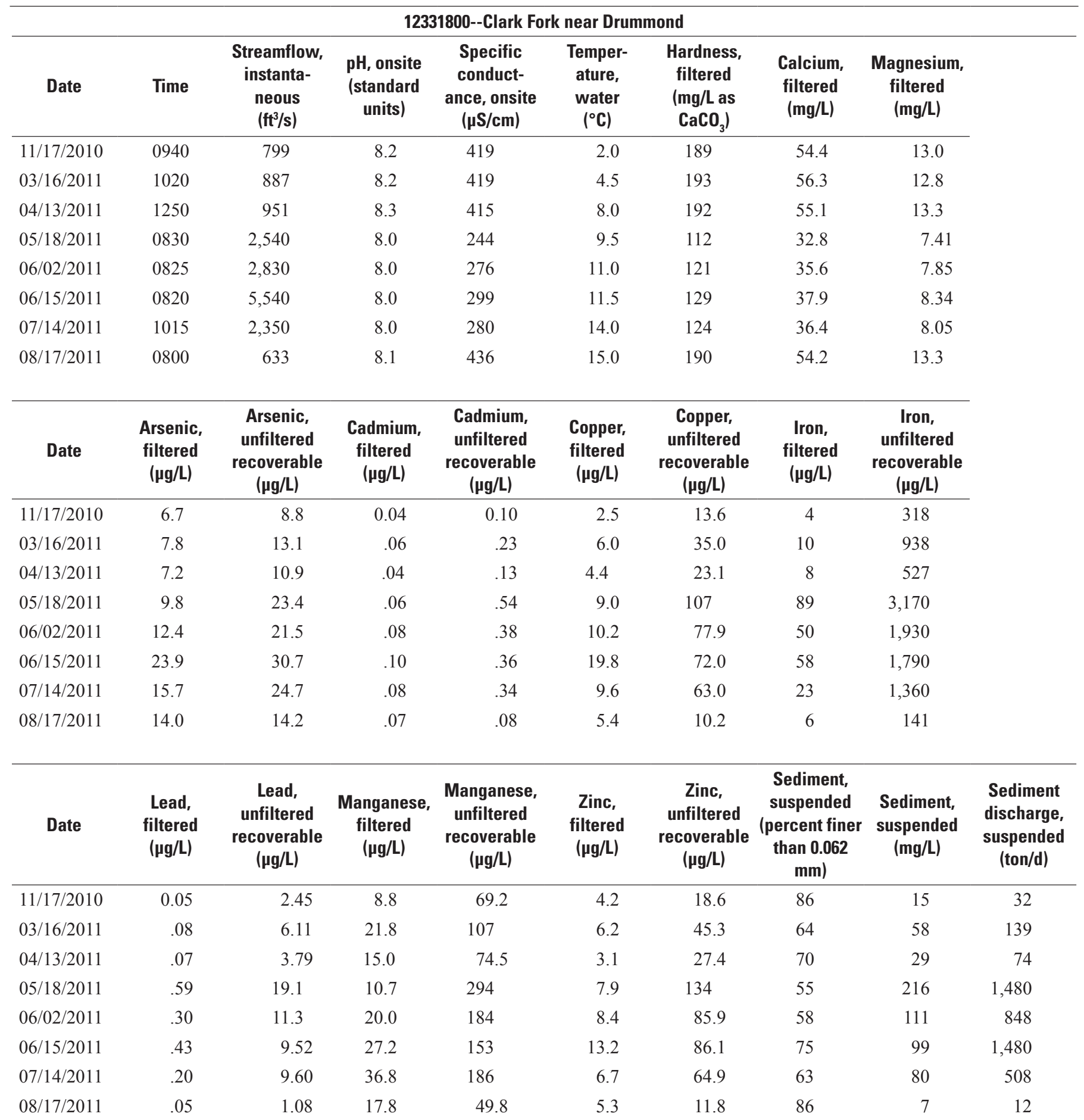


Table 4. Water-quality data for the Clark Fork basin, Montana, October 2010 through September 2011.-Continued

[Abbreviations: $\mathrm{CaCO}_{3}$, calcium carbonate; $\mathrm{ft}^{3} / \mathrm{s}$, cubic feet per second; ${ }^{\circ} \mathrm{C}$, degrees Celsius; E, estimated; lab, laboratory; $\mu \mathrm{g} / \mathrm{L}, \mathrm{micrograms}$ per liter; $\mu \mathrm{S} / \mathrm{cm}$, microsiemens per centimeter at $25^{\circ} \mathrm{C} ; \mathrm{mg} / \mathrm{L}$, milligrams per liter; mm, millimeters; NTRU, nephelometric turbidity ratio unit; ton/d, tons per day. Symbols: <, less than laboratory reporting level; --, no data]

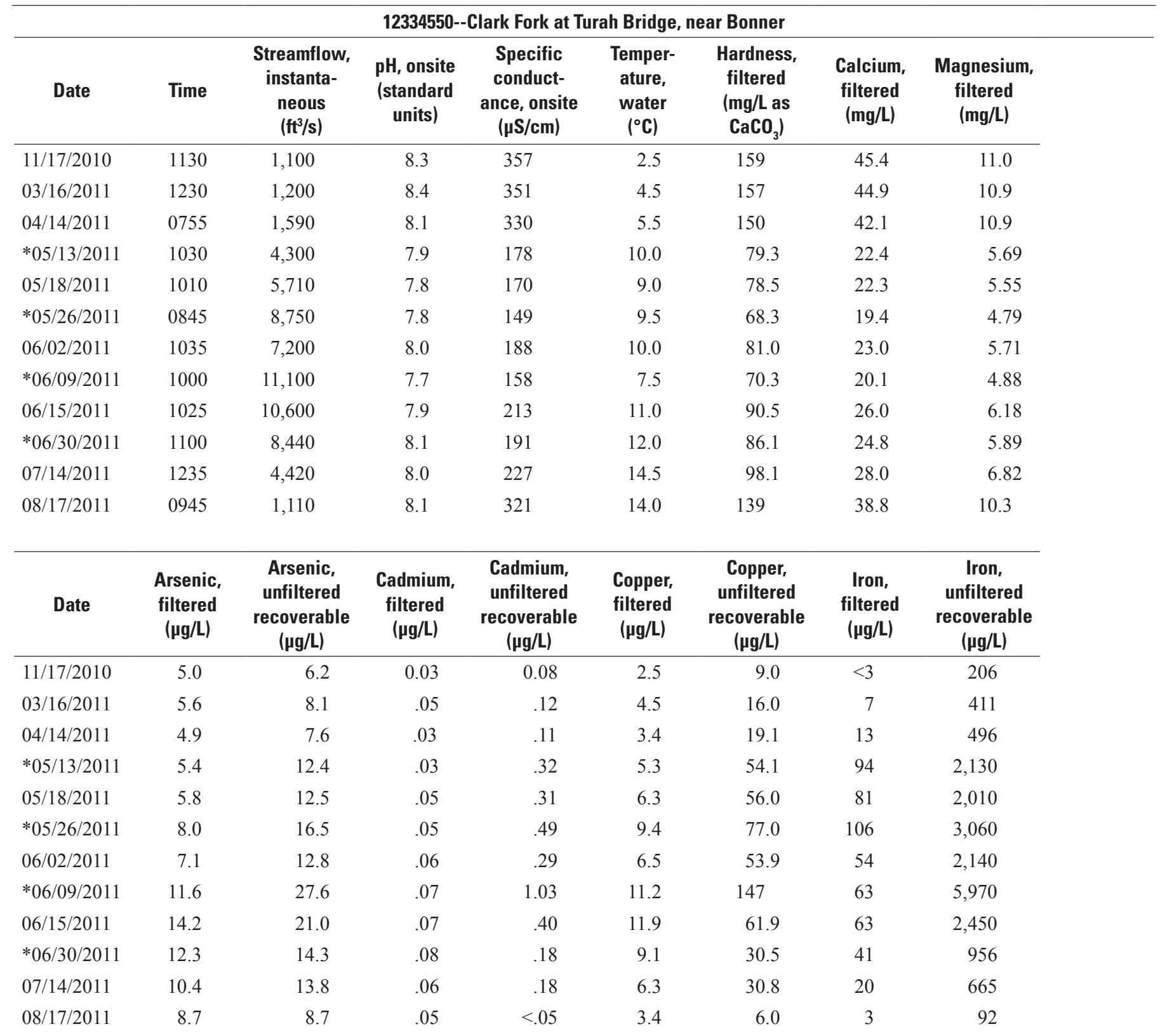


Table 4. Water-quality data for the Clark Fork basin, Montana, October 2010 through September 2011.—Continued

[Abbreviations: $\mathrm{CaCO}_{3}$, calcium carbonate; $\mathrm{ft} 3 / \mathrm{s}$, cubic feet per second; ${ }^{\circ} \mathrm{C}$, degrees Celsius; E, estimated; lab, laboratory; $\mu \mathrm{g} / \mathrm{L}$, micrograms per liter; $\mu \mathrm{S} / \mathrm{cm}$, microsiemens per centimeter at $25^{\circ} \mathrm{C} ; \mathrm{mg} / \mathrm{L}$, milligrams per liter; $\mathrm{mm}$, millimeters; NTRU, nephelometric turbidity ratio unit; ton/d, tons per day. Symbols: <, less than laboratory reporting level; --, no data]

\begin{tabular}{|c|c|c|c|c|c|c|c|c|c|}
\hline \multicolumn{10}{|c|}{ 12334550--Clark Fork at Turah Bridge, near Bonner } \\
\hline Date & $\begin{array}{c}\text { Lead, } \\
\text { filtered } \\
\text { ( } \mathrm{gg} / \mathrm{L})\end{array}$ & $\begin{array}{c}\text { Lead, } \\
\text { unfiltered } \\
\text { recoverable } \\
\text { ( } \mu \mathrm{g} / \mathrm{L})\end{array}$ & $\begin{array}{c}\text { Manganese, } \\
\text { filtered } \\
(\mu \mathrm{g} / \mathrm{L})\end{array}$ & $\begin{array}{l}\text { Manganese, } \\
\text { unfiltered } \\
\text { recoverable } \\
\text { ( } \mu \mathrm{g} / \mathrm{L})\end{array}$ & $\begin{array}{c}\text { Zinc, } \\
\text { filtered } \\
\text { ( } \mu \mathrm{g} / \mathrm{L})\end{array}$ & $\begin{array}{c}\text { Zinc, } \\
\text { unfiltered } \\
\text { recoverable } \\
(\mu \mathrm{g} / \mathrm{L})\end{array}$ & $\begin{array}{l}\text { Sediment, } \\
\text { suspended } \\
\text { (percent finer } \\
\text { than } 0.062 \\
\text { mm) }\end{array}$ & $\begin{array}{l}\text { Sediment, } \\
\text { suspended } \\
\text { (mg/L) }\end{array}$ & $\begin{array}{c}\text { Sediment } \\
\text { discharge, } \\
\text { suspended } \\
\text { (ton/d) }\end{array}$ \\
\hline $11 / 17 / 2010$ & 0.05 & 1.55 & 3.0 & 48.1 & 3.3 & 13.4 & 89 & 10 & 30 \\
\hline $04 / 14 / 2011$ & .06 & 3.14 & 8.6 & 64.2 & 3.7 & 26.2 & 81 & 24 & 103 \\
\hline$* 05 / 13 / 2011$ & .37 & 11.5 & 13.2 & 192 & 5.0 & 79.7 & 62 & 152 & 1,760 \\
\hline 05/18/2011 & .40 & 10.4 & 9.0 & 166 & 5.7 & 78.0 & 59 & 118 & 1,820 \\
\hline$* 05 / 26 / 2011$ & .41 & 14.2 & 17.2 & 245 & 9.0 & 117 & 55 & 245 & 5,790 \\
\hline $06 / 02 / 2011$ & .22 & 8.39 & 13.6 & 148 & 6.4 & 70.0 & 44 & 186 & 3,620 \\
\hline $07 / 14 / 2011$ & .13 & 3.89 & 22.5 & 95.9 & 4.9 & 34.5 & 60 & 40 & 477 \\
\hline 08/17/2011 & .03 & .51 & 9.2 & 26.1 & 3.8 & 7.5 & 73 & 5 & 15 \\
\hline
\end{tabular}


Table 4. Water-quality data for the Clark Fork basin, Montana, October 2010 through September 2011.—Continued

[Abbreviations: $\mathrm{CaCO}_{3}$, calcium carbonate; $\mathrm{ft}^{3} / \mathrm{s}$, cubic feet per second; ${ }^{\circ} \mathrm{C}$, degrees Celsius; E, estimated; lab, laboratory; $\mu \mathrm{g} / \mathrm{L}, \mathrm{micrograms}$ per liter; $\mu \mathrm{S} / \mathrm{cm}$, microsiemens per centimeter at $25^{\circ} \mathrm{C} ; \mathrm{mg} / \mathrm{L}$, milligrams per liter; mm, millimeters; NTRU, nephelometric turbidity ratio unit; ton/d, tons per day. Symbols: <, less than laboratory reporting level; --, no data]

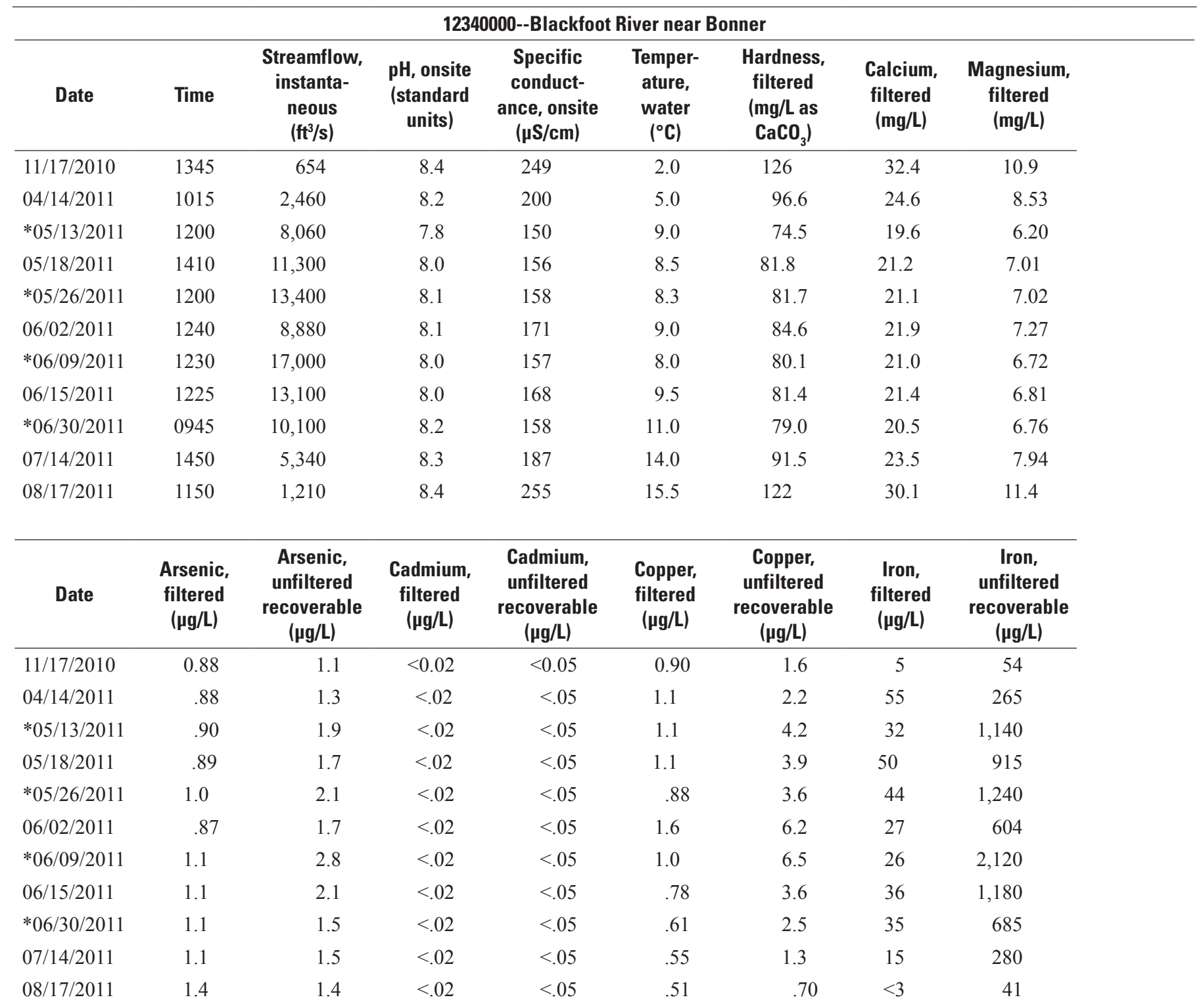


Table 4. Water-quality data for the Clark Fork basin, Montana, October 2010 through September 2011.-Continued

[Abbreviations: $\mathrm{CaCO}_{3}$, calcium carbonate; $\mathrm{ft}^{3} / \mathrm{s}$, cubic feet per second; ${ }^{\circ} \mathrm{C}$, degrees Celsius; E, estimated; lab, laboratory; $\mu \mathrm{g} / \mathrm{L}$, micrograms per liter; $\mu \mathrm{S} / \mathrm{cm}$, microsiemens per centimeter at $25^{\circ} \mathrm{C} ; \mathrm{mg} / \mathrm{L}$, milligrams per liter; $\mathrm{mm}$, millimeters; NTRU, nephelometric turbidity ratio unit; ton/d, tons per day. Symbols: <, less than laboratory reporting level; --, no data]

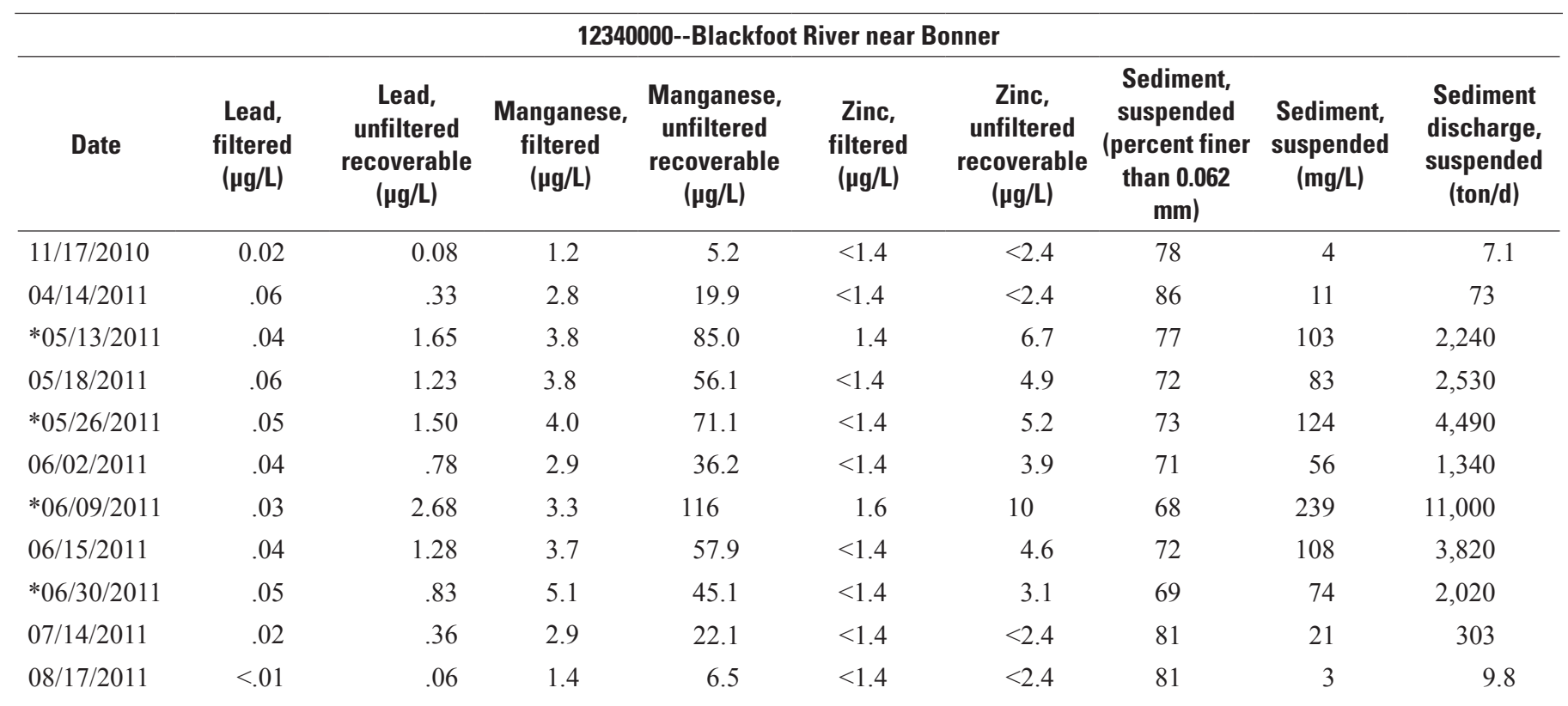


Table 4. Water-quality data for the Clark Fork basin, Montana, October 2010 through September 2011.-Continued

[Abbreviations: $\mathrm{CaCO}_{3}$, calcium carbonate; $\mathrm{ft}^{3} / \mathrm{s}$, cubic feet per second; ${ }^{\circ} \mathrm{C}$, degrees Celsius; E, estimated; lab, laboratory; $\mu \mathrm{g} / \mathrm{L}$, micrograms per liter; $\mu \mathrm{S} / \mathrm{cm}$, microsiemens per centimeter at $25^{\circ} \mathrm{C} ; \mathrm{mg} / \mathrm{L}$, milligrams per liter; mm, millimeters; NTRU, nephelometric turbidity ratio unit; ton/d, tons per day. Symbols: <, less than laboratory reporting level; --, no data]

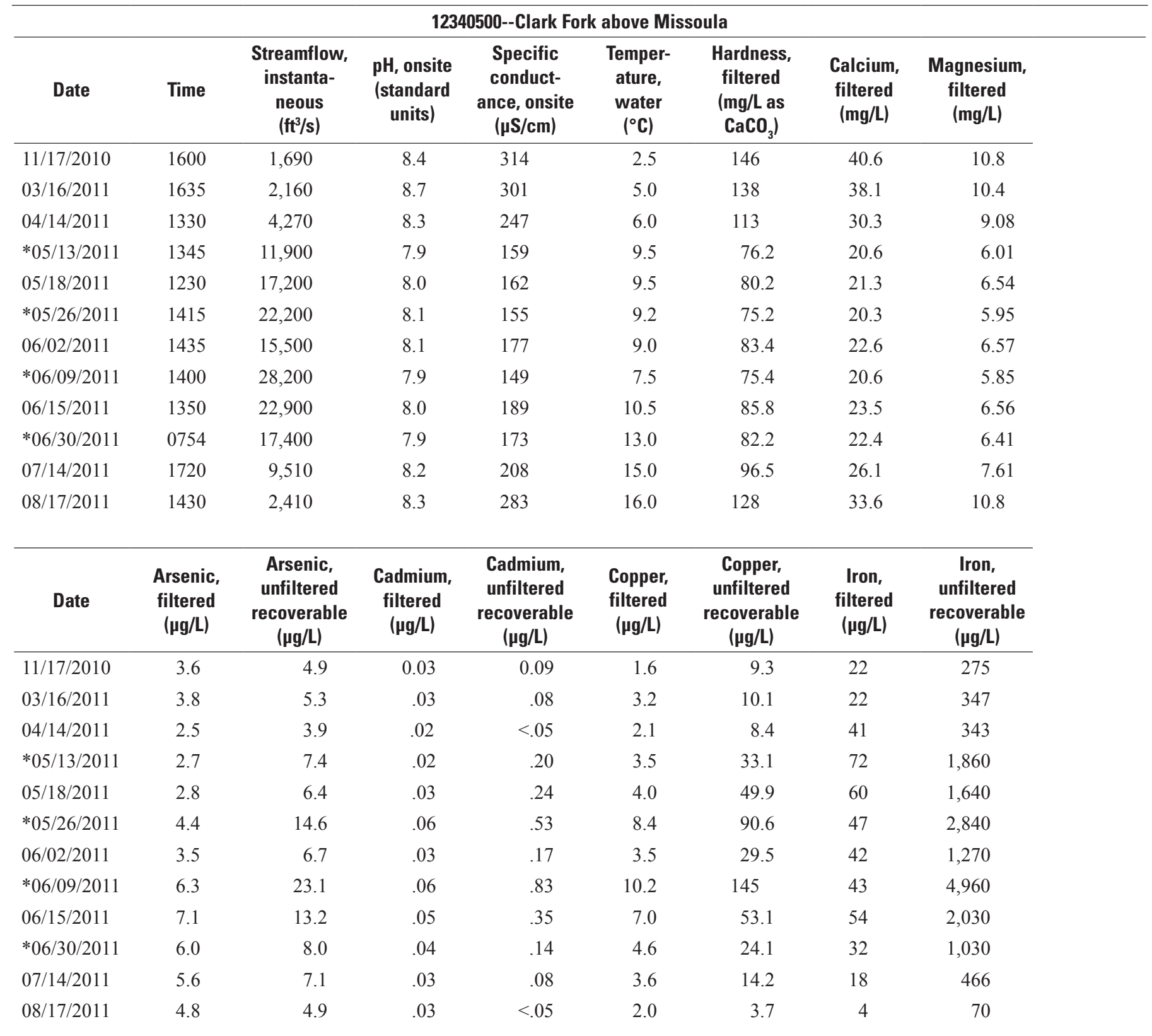


Table 4. Water-quality data for the Clark Fork basin, Montana, October 2010 through September 2011.-Continued

[Abbreviations: $\mathrm{CaCO}_{3}$, calcium carbonate; $\mathrm{ft}^{3} / \mathrm{s}$, cubic feet per second; ${ }^{\circ} \mathrm{C}$, degrees Celsius; E, estimated; lab, laboratory; $\mu \mathrm{g} / \mathrm{L}$, micrograms per liter; $\mu \mathrm{S} / \mathrm{cm}$, microsiemens per centimeter at $25^{\circ} \mathrm{C} ; \mathrm{mg} / \mathrm{L}$, milligrams per liter; $\mathrm{mm}$, millimeters; NTRU, nephelometric turbidity ratio unit; ton/d, tons per day. Symbols: <, less than laboratory reporting level; --, no data]

\begin{tabular}{|c|c|c|c|c|c|c|c|c|c|}
\hline \multicolumn{10}{|c|}{ 12340500--Clark Fork above Missoula } \\
\hline Date & $\begin{array}{c}\text { Lead, } \\
\text { filtered } \\
\text { ( } \mu \mathrm{g} / \mathrm{L})\end{array}$ & $\begin{array}{c}\text { Lead, } \\
\text { unfiltered } \\
\text { recoverable } \\
\text { ( } \mathrm{gg} / \mathrm{L})\end{array}$ & $\begin{array}{c}\text { Manganese, } \\
\text { filtered } \\
\text { ( } \mathrm{gg} / \mathrm{L})\end{array}$ & $\begin{array}{c}\text { Manganese, } \\
\text { unfiltered } \\
\text { recoverable } \\
(\mu \mathrm{g} / \mathrm{L})\end{array}$ & $\begin{array}{c}\text { Zinc, } \\
\text { filtered } \\
\text { ( } \mu \mathrm{g} / \mathrm{L})\end{array}$ & $\begin{array}{c}\text { Zinc, } \\
\text { unfiltered } \\
\text { recoverable } \\
(\mu \mathrm{g} / \mathrm{L})\end{array}$ & $\begin{array}{l}\text { Sediment, } \\
\text { suspended } \\
\text { (percent finer } \\
\text { than } 0.062 \\
\mathrm{~mm} \text { ) }\end{array}$ & $\begin{array}{c}\text { Sediment, } \\
\text { suspended } \\
\text { (mg/L) }\end{array}$ & $\begin{array}{l}\text { Sediment } \\
\text { discharge, } \\
\text { suspended } \\
\text { (ton/d) }\end{array}$ \\
\hline $11 / 17 / 2010$ & 0.03 & 1.60 & 6.6 & 38.7 & 2.7 & 17.1 & 71 & 17 & 78 \\
\hline $04 / 14 / 2011$ & .08 & 1.46 & 8.4 & 36.7 & $<1.4$ & 11.1 & 74 & 18 & 208 \\
\hline$* 05 / 13 / 2011$ & .19 & 6.96 & 11.8 & 141 & 3.4 & 47.7 & 50 & 206 & 6,620 \\
\hline 05/18/2011 & .21 & 7.48 & 9.1 & 109 & 3.4 & 46.3 & 64 & 128 & 5,940 \\
\hline$* 05 / 26 / 2011$ & .21 & 14.6 & 18.3 & 190 & 14.1 & 131 & 57 & 270 & 16,200 \\
\hline 06/02/2011 & .12 & 4.31 & 10.4 & 87.8 & 3.6 & 38.8 & 61 & 108 & 4,520 \\
\hline 07/14/2011 & .08 & 2.02 & 15.8 & 52.5 & 2.4 & 16.0 & 65 & 33 & 847 \\
\hline 08/17/2011 & .03 & .30 & 9.2 & 20.7 & 1.5 & 4.1 & 85 & 4 & 26 \\
\hline
\end{tabular}

"Sample collected as part of a supplemental sampling program. 
Table 5. Daily mean streamflow and suspended-sediment data for Clark Fork at Deer Lodge, Montana, October 2010 through September 2011.

[Abbreviations: acre-ft, acre-feet; $\mathrm{ft}^{3} / \mathrm{s}$, cubic feet per second; e, estimated; max, maximum; $\mathrm{mg} / \mathrm{L}$, milligrams per liter; min, minimum; ton/d, tons per day. Symbol: --, no data or value not computed]

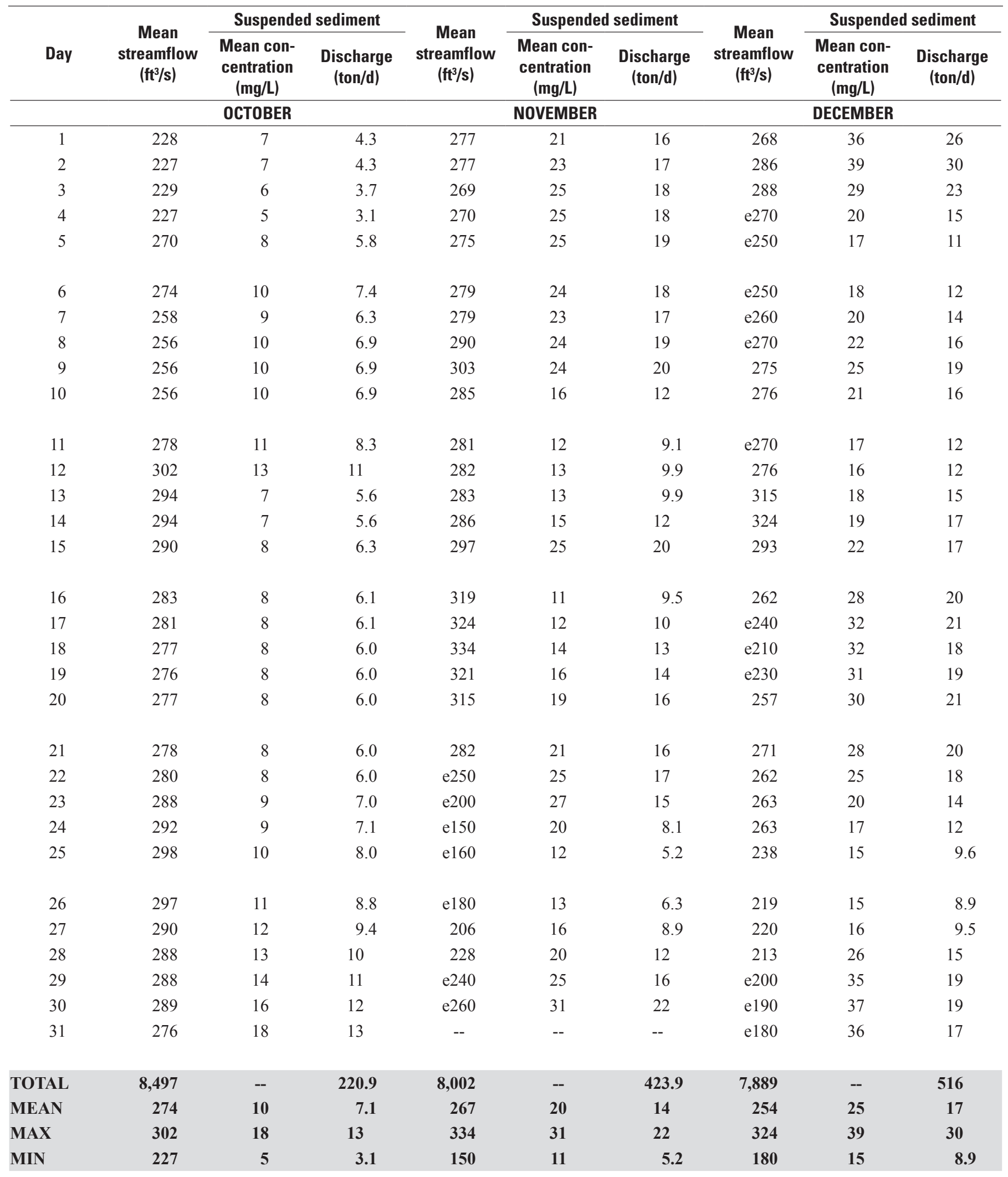


Table 5. Daily mean streamflow and suspended-sediment data for Clark Fork at Deer Lodge, Montana, October 2010 through September 2011.-Continued

[Abbreviations: acre-ft, acre-feet; $\mathrm{ft}^{3} / \mathrm{s}$, cubic feet per second; e, estimated; max, maximum; $\mathrm{mg} / \mathrm{L}$, milligrams per liter; min, minimum; ton/d, tons per day. Symbol: --, no data or value not computed]

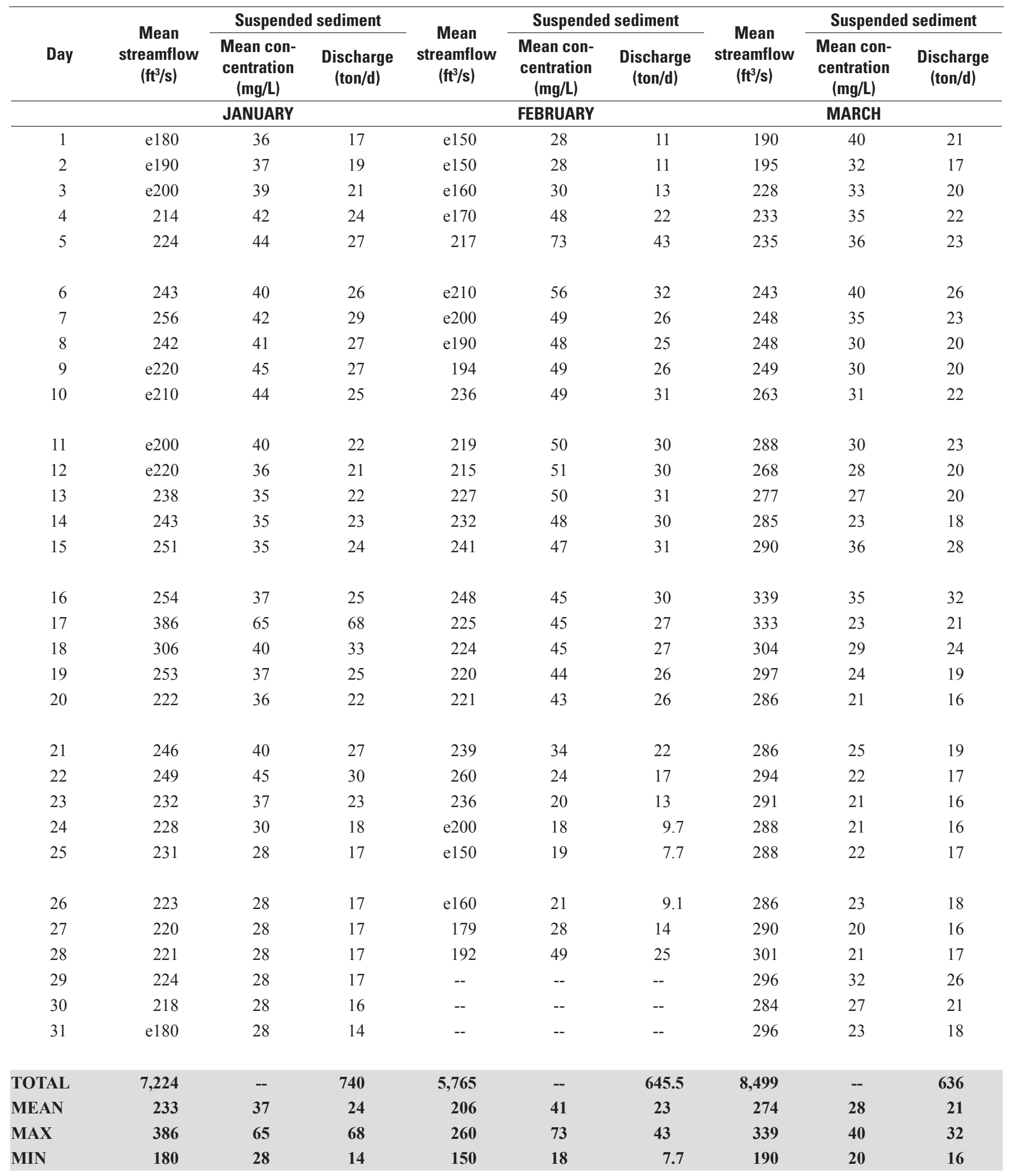


Table 5. Daily mean streamflow and suspended-sediment data for Clark Fork at Deer Lodge, Montana, October 2010 through September 2011.-Continued

[Abbreviations: acre-ft, acre-feet; $\mathrm{ft}^{3} / \mathrm{s}$, cubic feet per second; e, estimated; max, maximum; $\mathrm{mg} / \mathrm{L}$, milligrams per liter; min, minimum; ton/d, tons per day. Symbol: --, no data or value not computed]

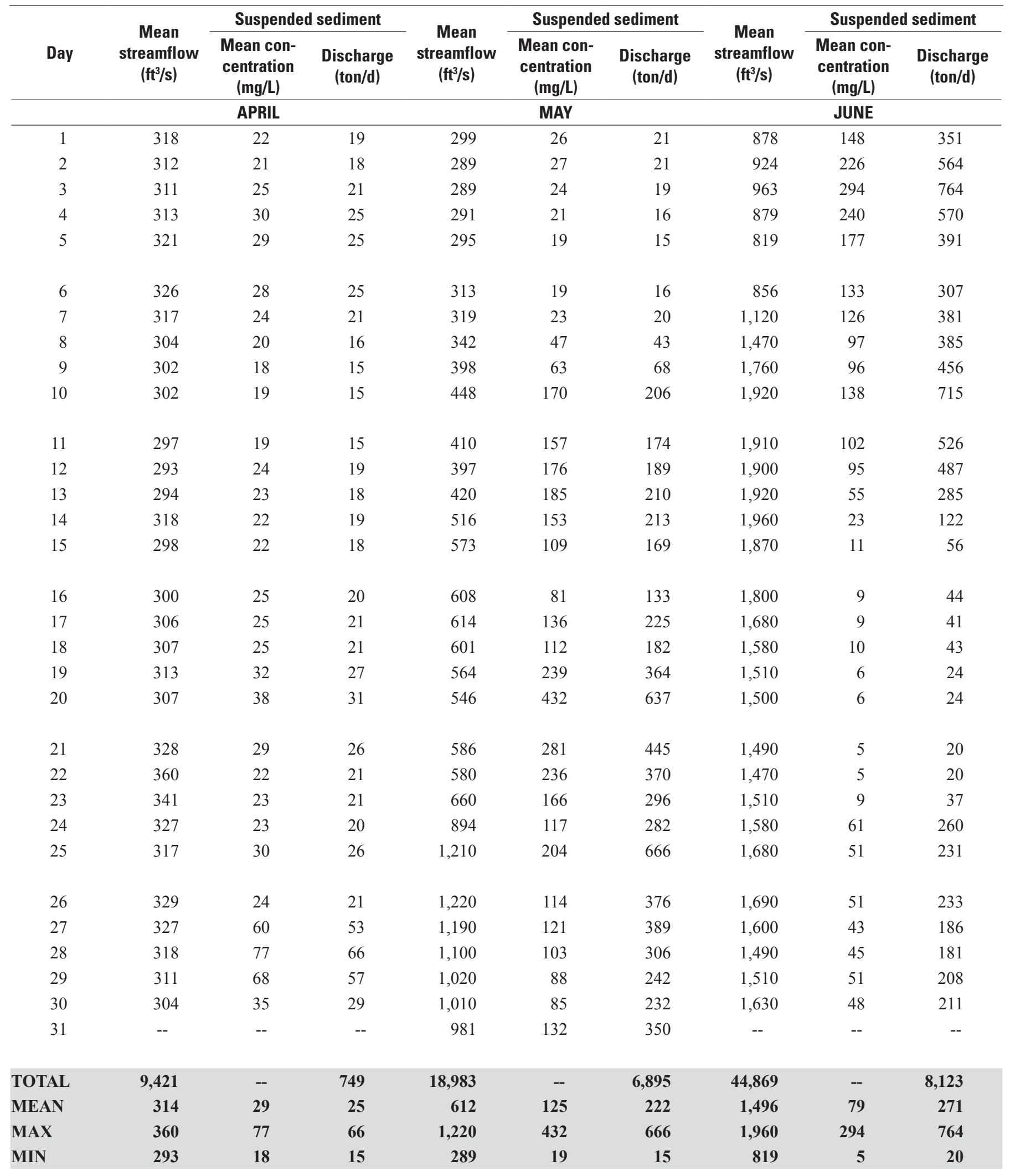


Table 5. Daily mean streamflow and suspended-sediment data for Clark Fork at Deer Lodge, Montana, October 2010 through September 2011.-Continued

[Abbreviations: acre-ft, acre-feet; $\mathrm{ft}^{3} / \mathrm{s}$, cubic feet per second; e, estimated; max, maximum; mg/L, milligrams per liter; min, minimum; ton/d, tons per day. Symbol: --, no data or value not computed]

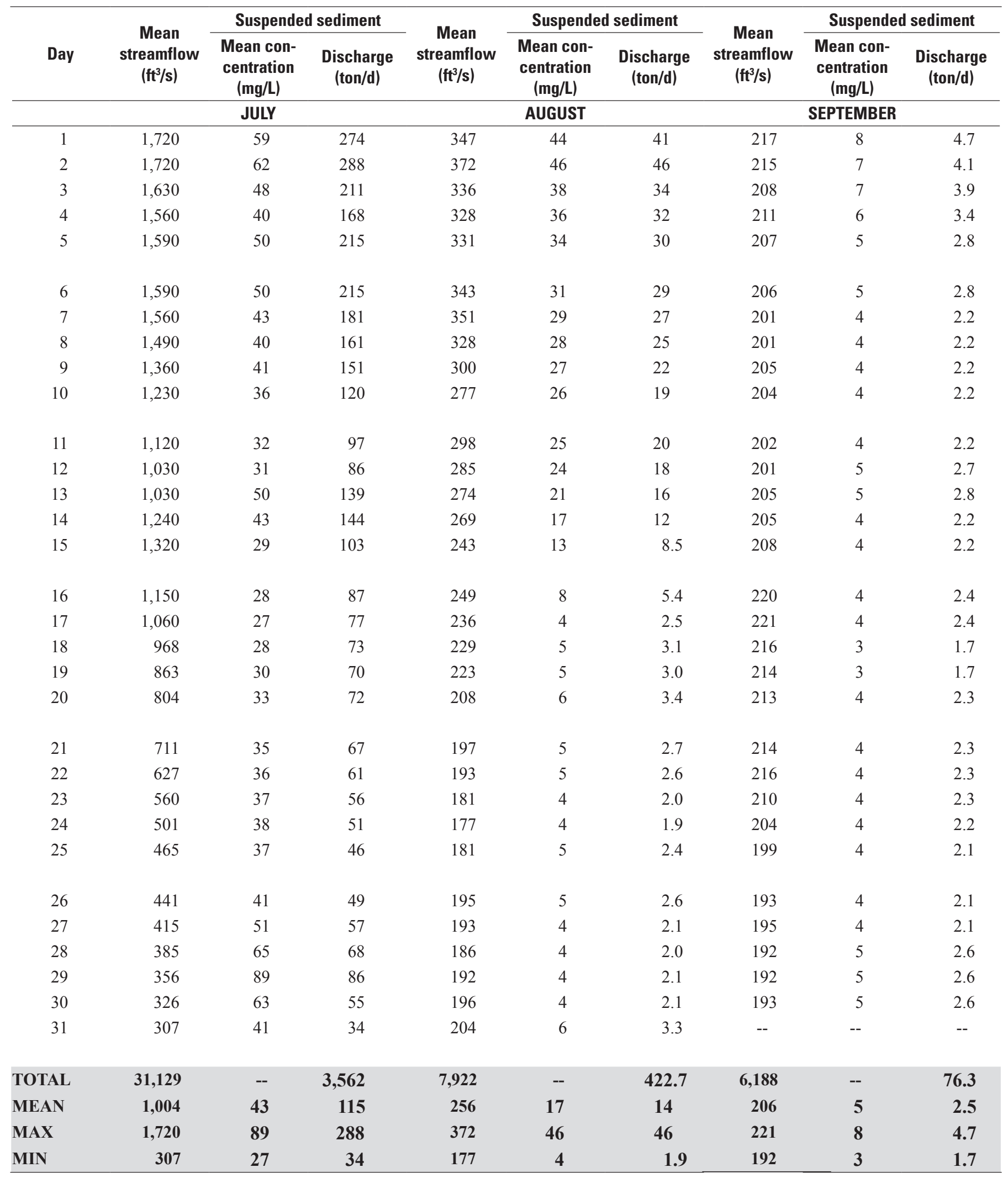

Total for water year 2011 (unrounded sum of daily values): streamflow-164,388 ft³/s (annual runoff-326,100 acre-ft); suspended-sediment discharge-23,010.3 tons. 
Table 6. Daily mean streamflow and suspended-sediment data for Clark Fork at Turah Bridge, near Bonner, Montana, October 2010 through September 2011.

[Abbreviations: acre-ft, acre-feet; $\mathrm{ft}^{3} / \mathrm{s}$, cubic feet per second; e, estimated; max, maximum; $\mathrm{mg} / \mathrm{L}$, milligrams per liter; min, minimum; ton/d, tons per day. Symbol: --, no data or value not computed]

\begin{tabular}{|c|c|c|c|c|c|c|c|c|c|}
\hline \multirow[b]{2}{*}{ Day } & \multirow[b]{2}{*}{$\begin{array}{c}\text { Mean } \\
\text { streamflow } \\
\left(\mathrm{ft}^{3} / \mathrm{s}\right)\end{array}$} & \multicolumn{2}{|c|}{ Suspended sediment } & \multirow[b]{2}{*}{$\begin{array}{c}\text { Mean } \\
\text { streamflow } \\
\left(\mathrm{ft}^{3} / \mathrm{s}\right)\end{array}$} & \multicolumn{2}{|c|}{ Suspended sediment } & \multirow[b]{2}{*}{$\begin{array}{c}\text { Mean } \\
\text { streamflow } \\
\left(\mathrm{ft}^{3} / \mathrm{s}\right)\end{array}$} & \multicolumn{2}{|c|}{ Suspended sediment } \\
\hline & & $\begin{array}{l}\text { Mean con- } \\
\text { centration } \\
(\mathrm{mg} / \mathrm{L})\end{array}$ & $\begin{array}{c}\text { Discharge } \\
\text { (ton/d) }\end{array}$ & & $\begin{array}{l}\text { Mean con- } \\
\text { centration } \\
(\mathrm{mg} / \mathrm{L})\end{array}$ & $\begin{array}{l}\text { Discharge } \\
\text { (ton/d) }\end{array}$ & & $\begin{array}{l}\text { Mean con- } \\
\text { centration } \\
(\mathrm{mg} / \mathrm{L})\end{array}$ & $\begin{array}{c}\text { Discharge } \\
\text { (ton/d) }\end{array}$ \\
\hline & & OCTOBER & & & NOVEMBER & & & DECEMBER & \\
\hline 1 & 910 & 4 & 9.8 & 1,010 & 6 & 16 & 871 & 5 & 12 \\
\hline 2 & 909 & 4 & 9.8 & 1,000 & 7 & 19 & e985 & 5 & 13 \\
\hline 3 & 905 & 5 & 12 & 1,000 & 7 & 19 & 1,000 & 5 & 14 \\
\hline 4 & 916 & 5 & 12 & 1,000 & 7 & 19 & 962 & 6 & 16 \\
\hline 5 & 964 & 10 & 26 & 1,000 & 7 & 19 & 903 & 6 & 15 \\
\hline 6 & 1,060 & 17 & 49 & 1,000 & 7 & 19 & 945 & 7 & 18 \\
\hline 7 & 1,070 & 16 & 46 & 1,020 & 7 & 19 & 983 & 8 & 21 \\
\hline 8 & 1,030 & 12 & 33 & 1,030 & 8 & 22 & 898 & 10 & 24 \\
\hline 9 & 1,040 & 12 & 34 & 1,050 & 8 & 23 & 1,010 & 11 & 30 \\
\hline 10 & 1,050 & 12 & 34 & 1,040 & 7 & 20 & 1,060 & 10 & 29 \\
\hline 11 & 1,060 & 12 & 34 & 1,010 & 6 & 16 & 980 & 9 & 24 \\
\hline 12 & 1,090 & 11 & 32 & 1,010 & 6 & 16 & 956 & 9 & 23 \\
\hline 13 & 1,090 & 10 & 29 & 1,000 & 5 & 14 & 995 & 8 & 21 \\
\hline 14 & 1,070 & 10 & 29 & 1,010 & 6 & 16 & 1,080 & 7 & 20 \\
\hline 15 & 1,060 & 9 & 26 & 1,010 & 6 & 16 & 1,090 & 6 & 18 \\
\hline 16 & 1,050 & 9 & 26 & 1,080 & 8 & 23 & 1,010 & 5 & 14 \\
\hline 17 & 1,040 & 8 & 22 & 1,110 & 10 & 30 & 971 & 4 & 10 \\
\hline 18 & 1,030 & 8 & 22 & 1,080 & 9 & 26 & e920 & 3 & 7.5 \\
\hline 19 & 1,030 & 7 & 19 & 1,070 & 8 & 23 & e920 & 4 & 9.9 \\
\hline 20 & 1,030 & 7 & 19 & 1,020 & 7 & 19 & e 880 & 4 & 9.5 \\
\hline 21 & 1,020 & 7 & 19 & 1,000 & 6 & 16 & e820 & 5 & 11 \\
\hline 22 & 1,020 & 8 & 22 & 870 & 4 & 9.4 & e840 & 4 & 9.1 \\
\hline 23 & 1,020 & 9 & 25 & 803 & 4 & 8.7 & e 800 & 4 & 8.6 \\
\hline 24 & 1,020 & 9 & 25 & 578 & 4 & 6.2 & e 820 & 3 & 6.6 \\
\hline 25 & 1,040 & 10 & 28 & 655 & 4 & 7.1 & e880 & 4 & 9.5 \\
\hline 26 & 1,060 & 10 & 29 & 647 & 5 & 8.7 & e840 & 5 & 11 \\
\hline 27 & 1,050 & 8 & 23 & 773 & 6 & 13 & e830 & 5 & 11 \\
\hline 28 & 1,030 & 7 & 19 & 917 & 6 & 15 & e880 & 6 & 14 \\
\hline 29 & 1,020 & 7 & 19 & 924 & 6 & 15 & e910 & 6 & 15 \\
\hline 30 & 1,010 & 7 & 19 & 915 & 7 & 17 & e915 & 6 & 15 \\
\hline 31 & 1,010 & 6 & 16 & -- & -- & -- & e820 & 5 & 11 \\
\hline TOTAL & 31,704 & -- & 767.6 & 28,632 & -- & 510.1 & 28,774 & -- & 470.7 \\
\hline MEAN & 1,023 & 9 & 25 & 954 & 6 & 17 & 928 & 6 & 15 \\
\hline MAX & 1,090 & 17 & 49 & 1,110 & 10 & 30 & 1,090 & 11 & 30 \\
\hline MIN & 905 & 4 & 9.8 & 578 & 4 & 6.2 & 800 & 3 & 6.6 \\
\hline
\end{tabular}


Table 6. Daily mean streamflow and suspended-sediment data for Clark Fork at Turah Bridge, near Bonner, Montana, 0 ctober 2010 through September 2011.-Continued

[Abbreviations: acre-ft, acre-feet; $\mathrm{ft}^{3} / \mathrm{s}$, cubic feet per second; e, estimated; max, maximum; $\mathrm{mg} / \mathrm{L}$, milligrams per liter; min, minimum; ton/d, tons per day. Symbol: --, no data or value not computed]

\begin{tabular}{|c|c|c|c|c|c|c|c|c|c|}
\hline \multirow[b]{2}{*}{ Day } & \multirow[b]{2}{*}{$\begin{array}{c}\text { Mean } \\
\text { streamflow } \\
\left(\mathrm{ft}^{3} / \mathrm{s}\right)\end{array}$} & \multicolumn{2}{|c|}{ Suspended sediment } & \multirow[b]{2}{*}{$\begin{array}{c}\text { Mean } \\
\text { streamflow } \\
\left(\mathrm{ft}^{3} / \mathbf{s}\right)\end{array}$} & \multicolumn{2}{|c|}{ Suspended sediment } & \multirow[b]{2}{*}{$\begin{array}{c}\text { Mean } \\
\text { streamflow } \\
\left(\mathrm{ft}^{3} / \mathbf{s}\right)\end{array}$} & \multicolumn{2}{|c|}{ Suspended sediment } \\
\hline & & $\begin{array}{l}\text { Mean con- } \\
\text { centration } \\
(\mathrm{mg} / \mathrm{L})\end{array}$ & $\begin{array}{c}\text { Discharge } \\
\text { (ton/d) }\end{array}$ & & $\begin{array}{c}\text { Mean con- } \\
\text { centration } \\
(\mathrm{mg} / \mathrm{L})\end{array}$ & $\begin{array}{c}\text { Discharge } \\
\text { (ton/d) }\end{array}$ & & $\begin{array}{l}\text { Mean con- } \\
\text { centration } \\
(\mathrm{mg} / \mathrm{L})\end{array}$ & $\begin{array}{c}\text { Discharge } \\
\text { (ton/d) }\end{array}$ \\
\hline 1 & e635 & 5 & 8.6 & 766 & 25 & 52 & 863 & 10 & 23 \\
\hline 2 & e755 & 6 & 12 & 710 & 24 & 46 & 879 & 9 & 21 \\
\hline 4 & e820 & 7 & 15 & 809 & 25 & 55 & 941 & 10 & 25 \\
\hline 5 & e850 & 7 & 16 & 984 & 39 & 104 & 962 & 12 & 31 \\
\hline 6 & e810 & 8 & 17 & 963 & 34 & 88 & 959 & 11 & 28 \\
\hline 7 & 943 & 11 & 28 & 963 & 27 & 70 & 975 & 9 & 24 \\
\hline 11 & 856 & 8 & 18 & 827 & 11 & 25 & 1,160 & 46 & 144 \\
\hline 12 & 969 & 7 & 18 & 940 & 16 & 41 & 1,120 & 42 & 127 \\
\hline 13 & 956 & 8 & 21 & 1,070 & 32 & 92 & 1,090 & 27 & 79 \\
\hline 14 & 894 & 8 & 19 & 1,250 & 45 & 152 & 1,160 & 27 & 85 \\
\hline 15 & 1,060 & 10 & 29 & 1,240 & 42 & 141 & 1,200 & 27 & 87 \\
\hline 16 & 1,130 & 23 & 70 & 1,500 & 59 & 239 & 1,250 & 31 & 105 \\
\hline 17 & 1,580 & 115 & 491 & 1,300 & 52 & 183 & 1,410 & 52 & 198 \\
\hline 18 & 2,130 & 137 & 788 & 1,110 & 39 & 117 & 1,280 & 34 & 118 \\
\hline 24 & 1,090 & 15 & 44 & 896 & 20 & 48 & 1,120 & 17 & 51 \\
\hline 25 & 1,070 & 16 & 46 & 717 & 15 & 29 & 1,140 & 17 & 52 \\
\hline 26 & 1,080 & 20 & 58 & 705 & 14 & 27 & 1,160 & 17 & 53 \\
\hline 27 & 1,050 & 24 & 68 & 763 & 15 & 31 & 1,140 & 18 & 55 \\
\hline 28 & 1,040 & 28 & 79 & 789 & 13 & 28 & 1,150 & 17 & 53 \\
\hline 29 & 1,030 & 31 & 86 & -- & -- & -- & 1,140 & 18 & 55 \\
\hline 30 & 1,040 & 33 & 93 & -- & -- & -- & 1,160 & 20 & 63 \\
\hline 31 & 919 & 30 & 74 & -- & -- & -- & 1,250 & 29 & 98 \\
\hline TOTAL & 32,902 & -- & $2,926.6$ & 26,225 & -- & 1,973 & 34,177 & -- & 1,979 \\
\hline MEAN & 1,061 & 25 & 94 & 937 & 26 & 70 & 1,102 & 21 & 64 \\
\hline MAX & 2,130 & 137 & 788 & 1,500 & 59 & 239 & 1,410 & 52 & 198 \\
\hline MIN & 635 & 5 & 8.6 & 705 & 11 & 23 & 863 & 9 & 21 \\
\hline
\end{tabular}


Table 6. Daily mean streamflow and suspended-sediment data for Clark Fork at Turah Bridge, near Bonner, Montana, October 2010 through September 2011.-Continued

[Abbreviations: acre-ft, acre-feet; $\mathrm{ft}^{3} / \mathrm{s}$, cubic feet per second; e, estimated; max, maximum; $\mathrm{mg} / \mathrm{L}$, milligrams per liter; min, minimum; ton/d, tons per day. Symbol: --, no data or value not computed]

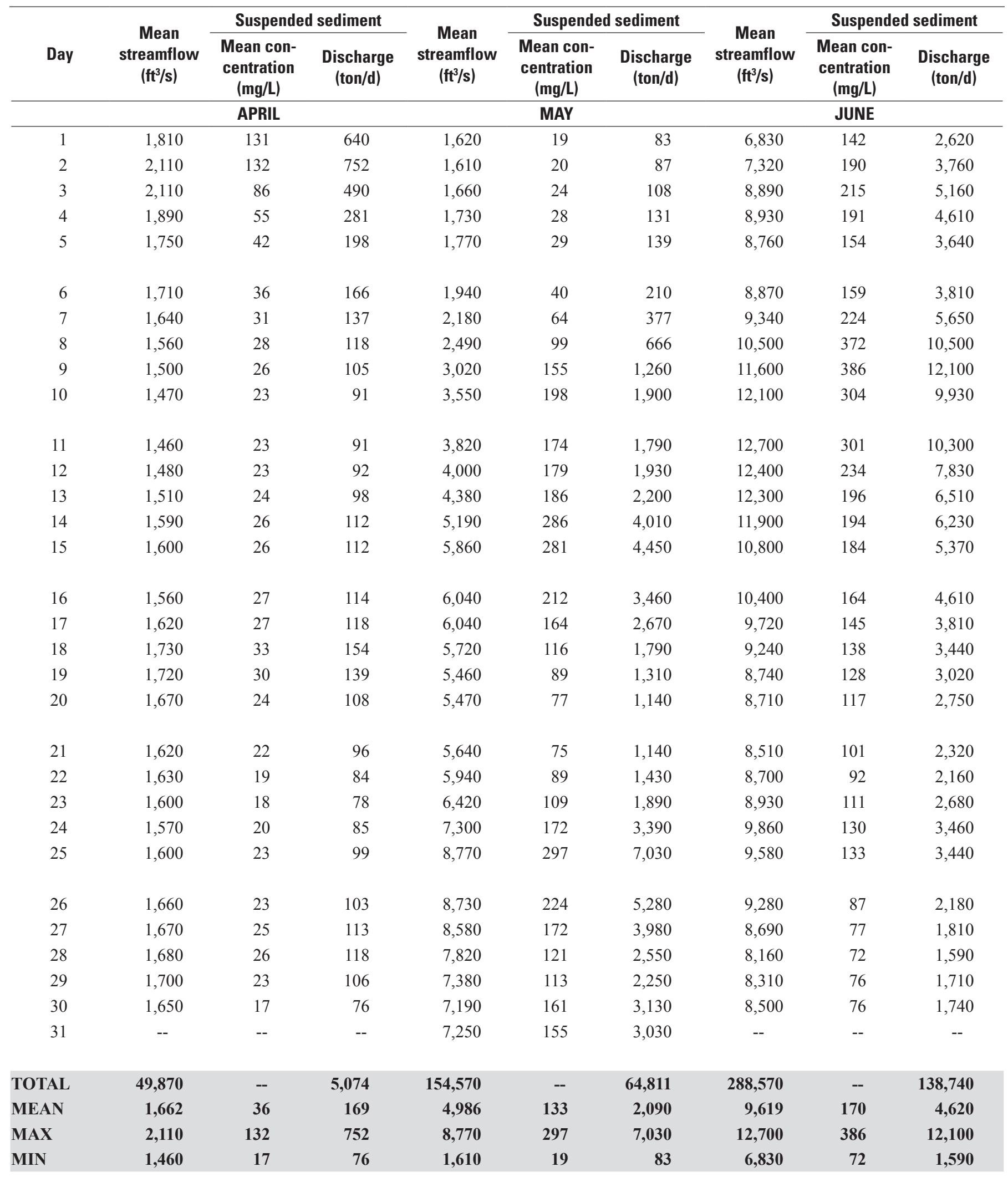


Table 6. Daily mean streamflow and suspended-sediment data for Clark Fork at Turah Bridge, near Bonner, Montana, 0 ctober 2010 through September 2011.-Continued

[Abbreviations: acre-ft, acre-feet; $\mathrm{ft}^{3} / \mathrm{s}$, cubic feet per second; e, estimated; max, maximum; $\mathrm{mg} / \mathrm{L}$, milligrams per liter; min, minimum; ton/d, tons per day. Symbol: --, no data or value not computed]

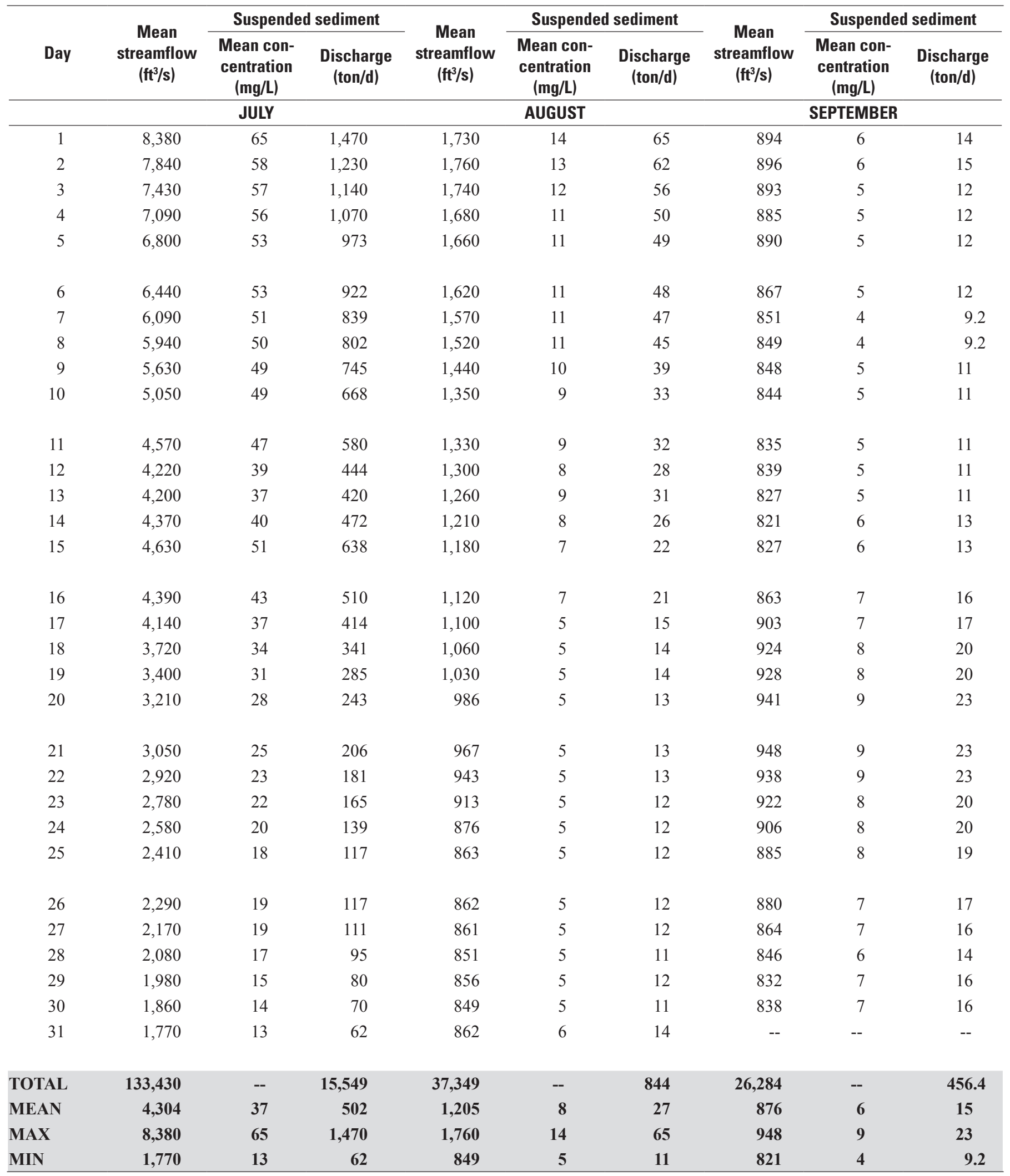

Total for water year 2011 (unrounded sum of daily values): streamflow-872,487 ft $3 / \mathrm{s}$ (annual runoff-1,731,000 acre-ft); suspended-sediment discharge-234,101.4 tons. 
Table 7. Daily mean streamflow and suspended-sediment data for Blackfoot River near Bonner, Montana, 0 ctober 2010 through September 2011.

[Abbreviations: acre-ft, acre-feet; $\mathrm{ft}^{3} / \mathrm{s}$, cubic feet per second; e, estimated; max, maximum; $\mathrm{mg} / \mathrm{L}$, milligrams per liter; min, minimum; ton/d, tons per day. Symbol: --, no data or value not computed]

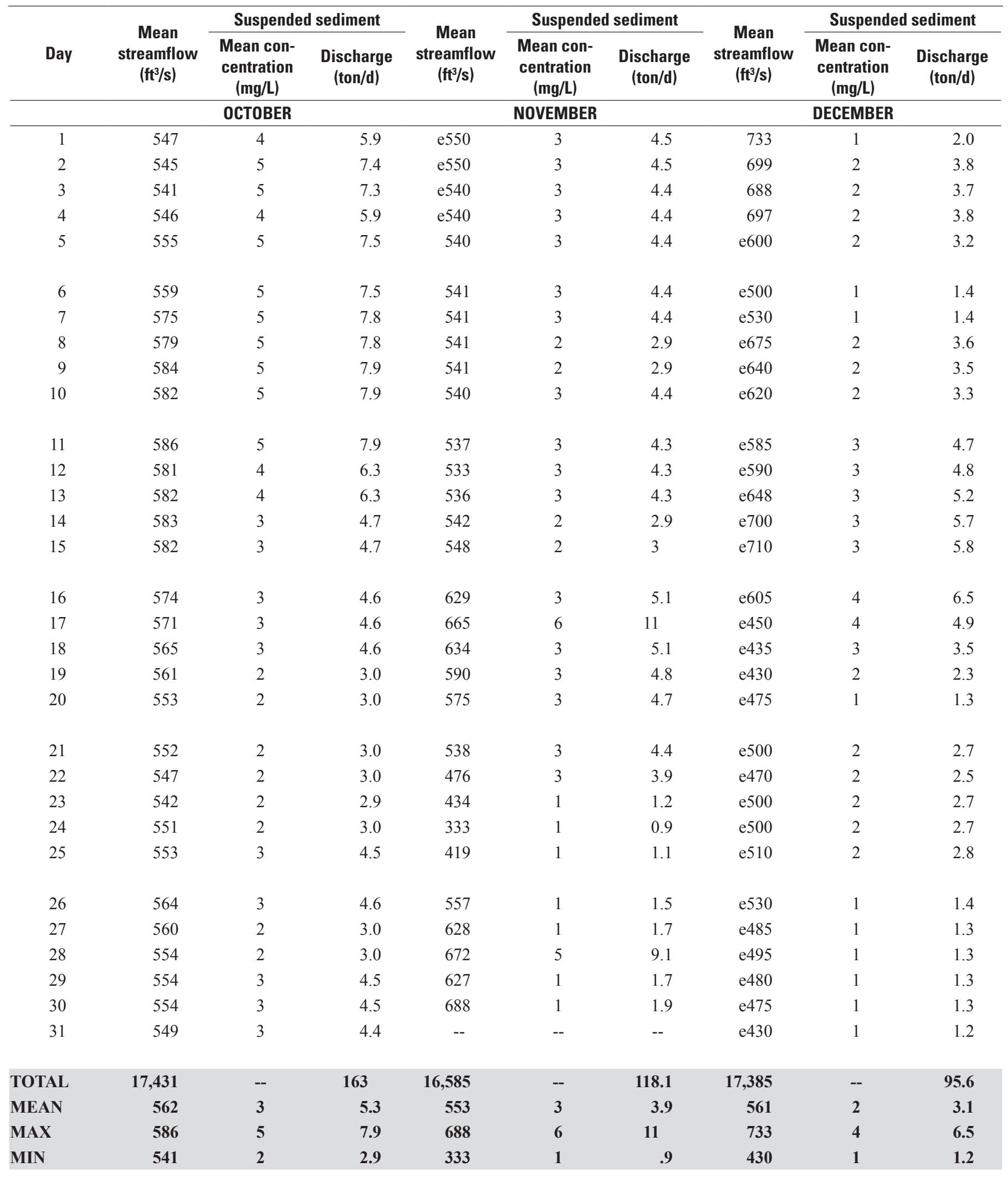


Table 7. Daily mean streamflow and suspended-sediment data for Blackfoot River near Bonner, Montana, 0 ctober 2010 through September 2011.-Continued

[Abbreviations: acre-ft, acre-feet; $\mathrm{ft}^{3} / \mathrm{s}$, cubic feet per second; e, estimated; max, maximum; $\mathrm{mg} / \mathrm{L}$, milligrams per liter; min, minimum; ton/d, tons per day. Symbol: --, no data or value not computed]

\begin{tabular}{|c|c|c|c|c|c|c|c|c|c|}
\hline \multirow[b]{2}{*}{ Day } & \multirow[b]{2}{*}{$\begin{array}{c}\text { Mean } \\
\text { streamflow } \\
\left(\mathrm{ft}^{3} / \mathrm{s}\right)\end{array}$} & \multicolumn{2}{|c|}{ Suspended sediment } & \multirow[b]{2}{*}{$\begin{array}{c}\text { Mean } \\
\text { streamflow } \\
\left(\mathrm{ft}^{3} / \mathrm{s}\right)\end{array}$} & \multicolumn{2}{|c|}{ Suspended sediment } & \multirow[b]{2}{*}{$\begin{array}{c}\text { Mean } \\
\text { streamflow } \\
\left(\mathrm{ft}^{3} / \mathrm{s}\right)\end{array}$} & \multicolumn{2}{|c|}{ Suspended sediment } \\
\hline & & $\begin{array}{l}\text { Mean con- } \\
\text { centration } \\
\text { (mg/L) }\end{array}$ & $\begin{array}{c}\text { Discharge } \\
\text { (ton/d) }\end{array}$ & & $\begin{array}{l}\text { Mean con- } \\
\text { centration } \\
(\mathrm{mg} / \mathrm{L})\end{array}$ & $\begin{array}{c}\text { Discharge } \\
\text { (ton/d) }\end{array}$ & & $\begin{array}{l}\text { Mean con- } \\
\text { centration } \\
\text { (mg/L) }\end{array}$ & $\begin{array}{c}\text { Discharge } \\
\text { (ton/d) }\end{array}$ \\
\hline 1 & e345 & 1 & 0.93 & 521 & 16 & 23 & 691 & 3 & 5.6 \\
\hline 2 & e395 & 1 & 1.1 & 529 & 9 & 13 & 681 & 3 & 5.5 \\
\hline 4 & e465 & 1 & 1.3 & 745 & 6 & 12 & 654 & 6 & 11 \\
\hline 5 & e480 & 2 & 2.6 & 807 & 6 & 13 & 638 & 9 & 16 \\
\hline 6 & e500 & 2 & 2.7 & 789 & 6 & 13 & 634 & 10 & 17 \\
\hline 7 & $\mathrm{e} 450$ & 2 & 2.4 & 792 & 6 & 13 & 624 & 9 & 15 \\
\hline 11 & e455 & 2 & 2.5 & 740 & 6 & 12 & 649 & 6 & 11 \\
\hline 12 & $\mathrm{e} 450$ & 2 & 2.4 & 742 & 7 & 14 & 648 & 5 & 8.7 \\
\hline 13 & e490 & 2 & 2.6 & 775 & 7 & 15 & 667 & 5 & 9.0 \\
\hline 14 & e500 & 3 & 4.0 & 781 & 7 & 15 & 730 & 8 & 16 \\
\hline 15 & e890 & 6 & 14 & 785 & 7 & 15 & 788 & 12 & 26 \\
\hline 16 & e965 & 12 & 31 & 854 & 12 & 28 & 835 & 13 & 29 \\
\hline 17 & 1,340 & 28 & 101 & 862 & 16 & 37 & 868 & 14 & 33 \\
\hline 18 & 1,280 & 20 & 69 & 846 & 9 & 21 & 894 & 15 & 36 \\
\hline 24 & 953 & 5 & 13 & 643 & 5 & 8.7 & 863 & 9 & 21 \\
\hline 25 & 944 & 5 & 13 & 538 & 4 & 5.8 & 921 & 10 & 25 \\
\hline 26 & 921 & 5 & 12 & 516 & 4 & 5.6 & 953 & 15 & 39 \\
\hline 27 & 887 & 5 & 12 & 639 & 4 & 6.9 & 972 & 18 & 47 \\
\hline 28 & 862 & 5 & 12 & 688 & 4 & 7.4 & 1,000 & 16 & 43 \\
\hline 29 & 857 & 14 & 32 & -- & -- & -- & 1,030 & 15 & 42 \\
\hline 30 & 855 & 20 & 46 & -- & -- & -- & 1,110 & 16 & 48 \\
\hline 31 & 660 & 10 & 18 & -- & -- & -- & 1,550 & 33 & 138 \\
\hline TOTAL & 23,364 & -- & 547.63 & 20,003 & -- & 372.9 & 25,150 & -- & 774.6 \\
\hline MEAN & 754 & 7 & 18 & 714 & 7 & 13 & 811 & 10 & 25 \\
\hline MAX & 1,340 & 28 & 101 & 862 & 16 & 37 & 1,550 & 33 & 138 \\
\hline MIN & 345 & 1 & .93 & 516 & 4 & 5.6 & 620 & 3 & 5.0 \\
\hline
\end{tabular}


Table 7. Daily mean streamflow and suspended-sediment data for Blackfoot River near Bonner, Montana, 0 ctober 2010 through September 2011.-Continued

[Abbreviations: acre-ft, acre-feet; $\mathrm{ft}^{3} / \mathrm{s}$, cubic feet per second; e, estimated; max, maximum; $\mathrm{mg} / \mathrm{L}$, milligrams per liter; min, minimum; ton/d, tons per day. Symbol: --, no data or value not computed]

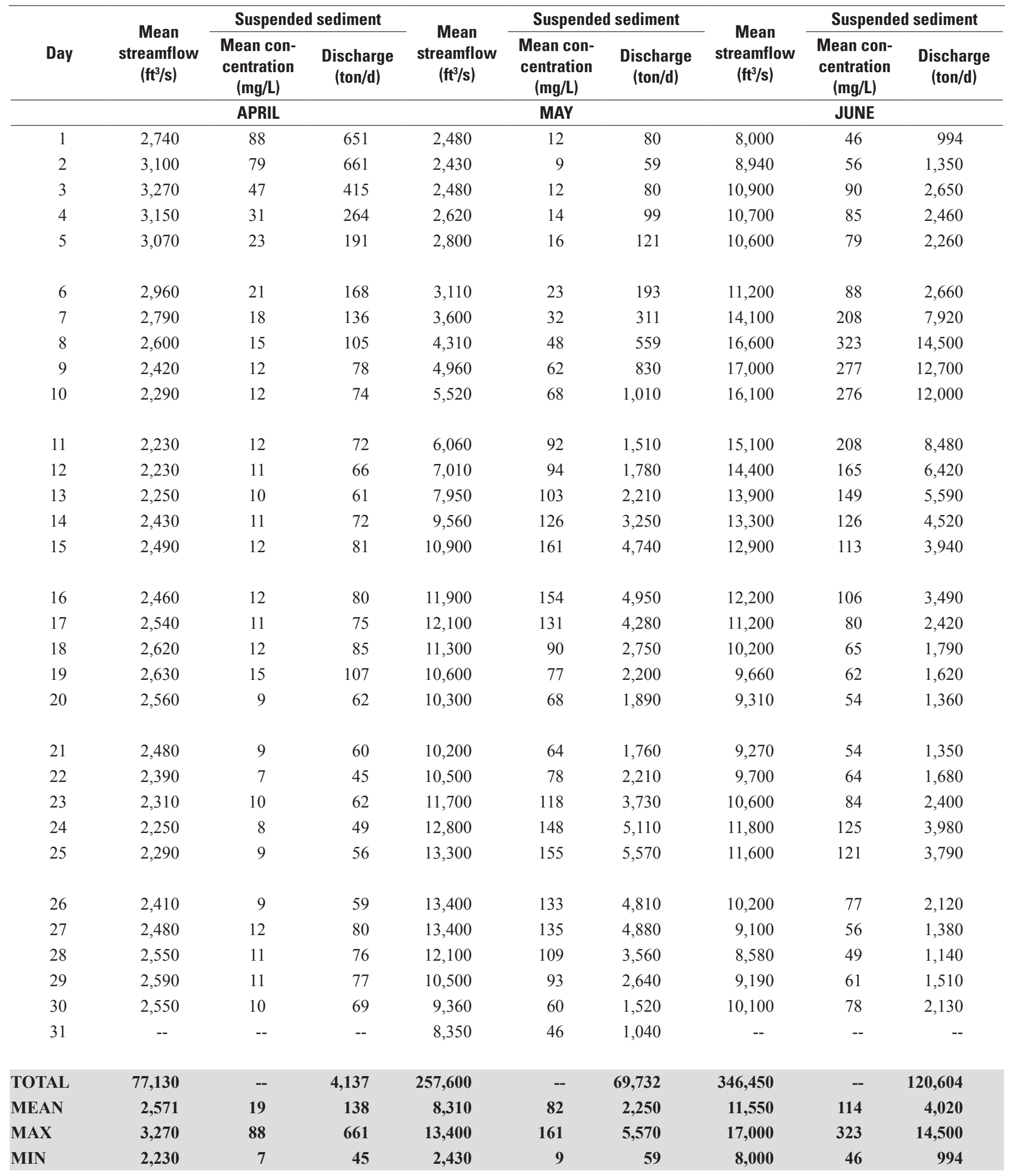


Table 7. Daily mean streamflow and suspended-sediment data for Blackfoot River near Bonner, Montana, 0 ctober 2010 through September 2011.-Continued

[Abbreviations: acre-ft, acre-feet; $\mathrm{ft}^{3} / \mathrm{s}$, cubic feet per second; e, estimated; max, maximum; $\mathrm{mg} / \mathrm{L}$, milligrams per liter; min, minimum; ton/d, tons per day. Symbol: --, no data or value not computed]

\begin{tabular}{|c|c|c|c|c|c|c|c|c|c|}
\hline \multirow{3}{*}{ Day } & \multirow[b]{2}{*}{$\begin{array}{c}\text { Mean } \\
\text { streamflow } \\
\left(\mathrm{ft}^{3} / \mathrm{s}\right)\end{array}$} & \multicolumn{2}{|c|}{ Suspended sediment } & \multirow[b]{2}{*}{$\begin{array}{c}\text { Mean } \\
\text { streamflow } \\
\left(\mathrm{ft}^{3} / \mathrm{s}\right)\end{array}$} & \multicolumn{2}{|c|}{ Suspended sediment } & \multirow[b]{2}{*}{$\begin{array}{c}\text { Mean } \\
\text { streamflow } \\
\left(\mathrm{ft}^{3} / \mathrm{s}\right)\end{array}$} & \multicolumn{2}{|c|}{ Suspended sediment } \\
\hline & & $\begin{array}{l}\text { Mean con- } \\
\text { centration } \\
\text { (mg/L) }\end{array}$ & $\begin{array}{l}\text { Discharge } \\
\text { (ton/d) }\end{array}$ & & $\begin{array}{l}\text { Mean con- } \\
\text { centration } \\
(\mathrm{mg} / \mathrm{L})\end{array}$ & $\begin{array}{c}\text { Discharge } \\
\text { (ton/d) }\end{array}$ & & $\begin{array}{l}\text { Mean con- } \\
\text { centration } \\
(\mathrm{mg} / \mathrm{L})\end{array}$ & $\begin{array}{c}\text { Discharge } \\
\text { (ton/d) }\end{array}$ \\
\hline & \multicolumn{3}{|c|}{ JULY } & \multicolumn{3}{|c|}{ AUGUST } & \multicolumn{3}{|c|}{ SEPTEMBER } \\
\hline 1 & 9,850 & 67 & 1,780 & 1,830 & 5 & 25 & 934 & 4 & 10 \\
\hline 2 & 8,890 & 49 & 1,180 & 1,790 & 5 & 24 & 943 & 3 & 7.6 \\
\hline 4 & 8,020 & 43 & 931 & 1,710 & 4 & 18 & 905 & 2 & 4.9 \\
\hline 5 & 7,800 & 39 & 821 & 1,650 & 4 & 18 & 888 & 2 & 4.8 \\
\hline 6 & 7,430 & 37 & 742 & 1,610 & 5 & 22 & 869 & 2 & 4.7 \\
\hline 7 & 7,080 & 34 & 650 & 1,580 & 5 & 21 & 844 & 2 & 4.6 \\
\hline 11 & 5,300 & 26 & 372 & 1,380 & 3 & 11 & 809 & 3 & 6.6 \\
\hline 12 & 5,080 & 24 & 329 & 1,370 & 3 & 11 & 794 & 3 & 6.4 \\
\hline 13 & 5,240 & 25 & 354 & 1,320 & 3 & 11 & 792 & 3 & 6.4 \\
\hline 14 & 5,270 & 21 & 299 & 1,290 & 3 & 10 & 781 & 3 & 6.3 \\
\hline 15 & 4,960 & 19 & 254 & 1,260 & 4 & 14 & 771 & 2 & 4.2 \\
\hline 16 & 4,560 & 16 & 197 & 1,230 & 4 & 13 & 786 & 2 & 4.2 \\
\hline 17 & 4,310 & 14 & 163 & 1,200 & 3 & 9.7 & 787 & 2 & 4.2 \\
\hline 18 & 4,040 & 13 & 142 & 1,150 & 3 & 9.3 & 785 & 2 & 4.2 \\
\hline 24 & 2,800 & 7 & 53 & 1,030 & 3 & 8.3 & 734 & 3 & 5.9 \\
\hline 25 & 2,630 & 7 & 50 & 998 & 3 & 8.1 & 720 & 3 & 5.8 \\
\hline 26 & 2,490 & 7 & 47 & 976 & 3 & 7.9 & 711 & 3 & 5.8 \\
\hline 27 & 2,360 & 8 & 51 & 951 & 3 & 7.7 & 704 & 4 & 7.6 \\
\hline 28 & 2,240 & 7 & 42 & 938 & 4 & 10 & 697 & 4 & 7.5 \\
\hline 29 & 2,130 & 6 & 35 & 932 & 3 & 7.5 & 686 & 3 & 5.6 \\
\hline 30 & 2,020 & 6 & 33 & 926 & 3 & 7.5 & 685 & 3 & 5.5 \\
\hline 31 & 1,940 & 5 & 26 & 929 & 3 & 7.5 & -- & -- & -- \\
\hline TOTAL & 150,710 & -- & 11,344 & 39,720 & -- & 392.2 & 24,005 & -- & 182.7 \\
\hline MEAN & 4,862 & 21 & 366 & 1,281 & 4 & 13 & 800 & 3 & 6.1 \\
\hline MAX & 9,850 & 67 & 1,780 & 1,830 & 5 & 25 & 943 & 4 & 10 \\
\hline MIN & 1,940 & 5 & 26 & 926 & 3 & 7.5 & 685 & 2 & 4.2 \\
\hline
\end{tabular}

Total for water year 2011 (unrounded sum of daily values): streamflow-1,015,533 ft $3 / \mathrm{s}$ (annual runoff-2,014,000 acre-ft); suspended-sediment discharge-208,463.7 tons. 
Table 8. Daily mean streamflow and suspended-sediment data for Clark Fork above Missoula, Montana, October 2010 through September 2011.

[Abbreviations: acre-ft, acre-feet; $\mathrm{ft}^{3} / \mathrm{s}$, cubic feet per second; e, estimated; max, maximum; $\mathrm{mg} / \mathrm{L}$, milligrams per liter; min, minimum; ton/d, tons per day. Symbol: --, no data or value not computed]

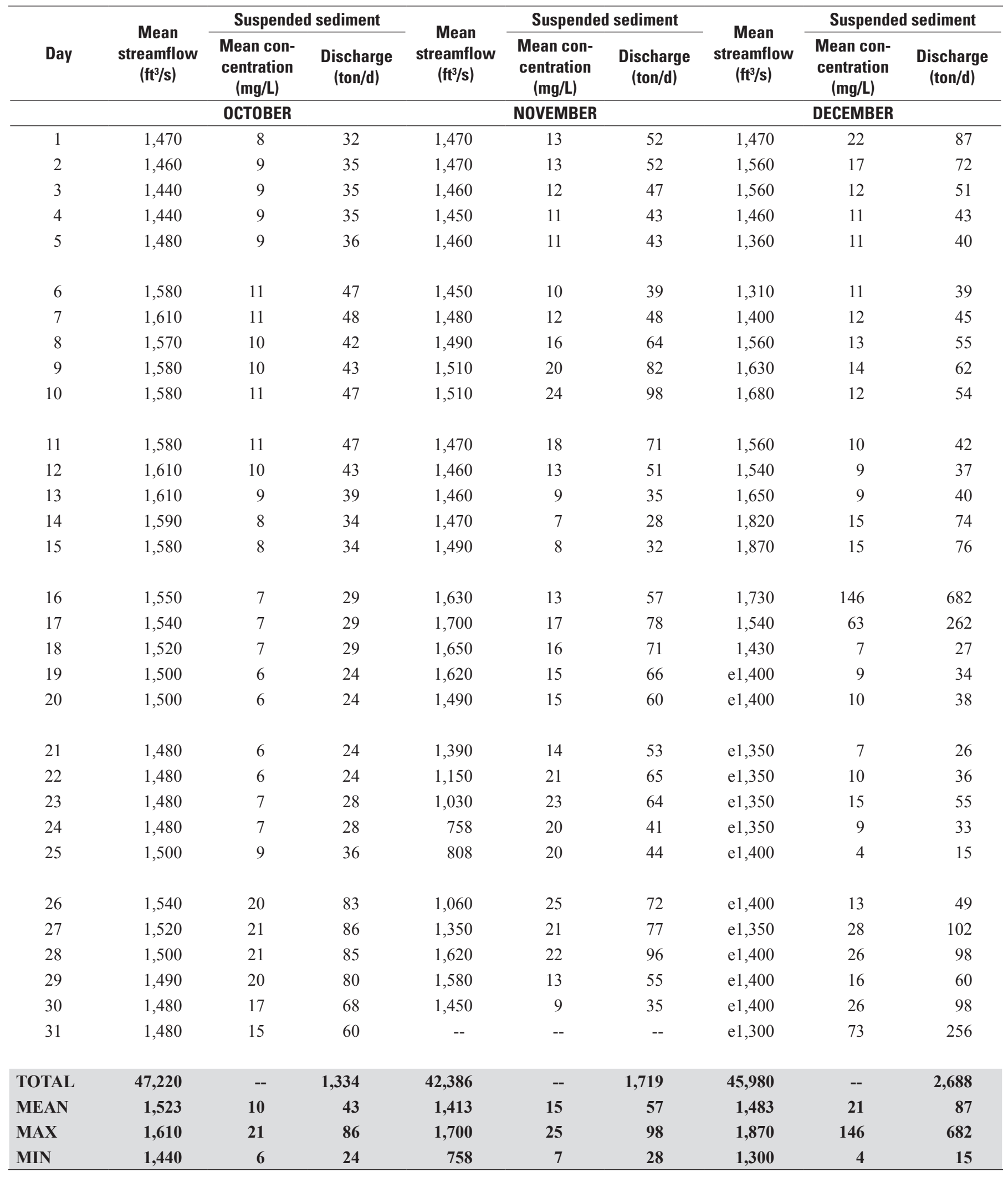


Table 8. Daily mean streamflow and suspended-sediment data for Clark Fork above Missoula, Montana, October 2010 through September 2011.-Continued

[Abbreviations: acre-ft, acre-feet; $\mathrm{ft}^{3} / \mathrm{s}$, cubic feet per second; e, estimated; max, maximum; $\mathrm{mg} / \mathrm{L}$, milligrams per liter; min, minimum; ton/d, tons per day. Symbol: --, no data or value not computed]

\begin{tabular}{|c|c|c|c|c|c|c|c|c|c|}
\hline \multirow[b]{2}{*}{ Day } & \multirow{2}{*}{$\begin{array}{c}\text { Mean } \\
\text { streamflow } \\
\left(\mathrm{ft}^{3} / \mathrm{s}\right)\end{array}$} & \multicolumn{2}{|c|}{ Suspended sediment } & \multirow[b]{2}{*}{$\begin{array}{c}\text { Mean } \\
\text { streamflow } \\
\left(\mathrm{ft}^{3} / \mathrm{s}\right)\end{array}$} & \multicolumn{2}{|c|}{ Suspended sediment } & \multirow[b]{2}{*}{$\begin{array}{c}\text { Mean } \\
\text { streamflow } \\
\left(\mathrm{ft}^{3} / \mathrm{s}\right)\end{array}$} & \multicolumn{2}{|c|}{ Suspended sediment } \\
\hline & & $\begin{array}{c}\text { Mean con- } \\
\text { centration } \\
(\mathrm{mg} / \mathrm{L})\end{array}$ & $\begin{array}{l}\text { Discharge } \\
\text { (ton/d) }\end{array}$ & & $\begin{array}{c}\text { Mean con- } \\
\text { centration } \\
(\mathrm{mg} / \mathrm{L})\end{array}$ & $\begin{array}{l}\text { Discharge } \\
\text { (ton/d) }\end{array}$ & & $\begin{array}{c}\text { Mean con- } \\
\text { centration } \\
(\mathrm{mg} / \mathrm{L})\end{array}$ & $\begin{array}{c}\text { Discharge } \\
\text { (ton/d) }\end{array}$ \\
\hline & & JANUARY & & & FEBRUARY & & & MARCH & \\
\hline 1 & e1,100 & 161 & 478 & 1,290 & 16 & 56 & 1,570 & 7 & 30 \\
\hline 2 & $\mathrm{e} 1,200$ & 179 & 580 & 1,270 & 22 & 75 & 1,580 & 7 & 30 \\
\hline 4 & $\mathrm{e} 1,350$ & 53 & 193 & 1,640 & 74 & 328 & 1,620 & 8 & 35 \\
\hline 5 & $\mathrm{e} 1,400$ & 25 & 95 & 1,900 & 98 & 503 & 1,630 & 7 & 31 \\
\hline 6 & $\mathrm{e} 1,400$ & 41 & 155 & 1,850 & 83 & 415 & 1,620 & 7 & 31 \\
\hline 7 & $\mathrm{e} 1,450$ & 80 & 313 & 1,880 & 67 & 340 & 1,620 & 6 & 26 \\
\hline 11 & $\mathrm{e} 1,400$ & 128 & 484 & 1,620 & 9 & 39 & 1,820 & 25 & 123 \\
\hline 12 & $\mathrm{e} 1,450$ & 189 & 740 & 1,780 & 13 & 62 & 1,810 & 19 & 93 \\
\hline 13 & $\mathrm{e} 1,500$ & 221 & 895 & 1,950 & 16 & 84 & 1,780 & 16 & 77 \\
\hline 14 & e1,600 & 118 & 510 & 2,190 & 23 & 136 & 1,910 & 18 & 93 \\
\hline 15 & $\mathrm{e} 2,000$ & 48 & 259 & 2,180 & 26 & 153 & 2,040 & 19 & 105 \\
\hline 16 & e2,100 & 54 & 306 & 2,560 & 49 & 339 & 2,130 & 18 & 104 \\
\hline 17 & 2,980 & 116 & 933 & 2,410 & 44 & 286 & 2,370 & 32 & 205 \\
\hline 18 & 3,850 & 132 & 1,370 & 2,130 & 27 & 155 & 2,270 & 25 & 153 \\
\hline 24 & 2,220 & 10 & 60 & 1,580 & 12 & 51 & 2,020 & 13 & 71 \\
\hline 25 & 2,190 & 9 & 53 & 1,230 & 3 & 10 & 2,100 & 15 & 85 \\
\hline 26 & 2,170 & 11 & 64 & 1,130 & 3 & 9.2 & 2,160 & 17 & 99 \\
\hline 27 & 2,090 & 13 & 73 & 1,350 & 8 & 29 & 2,150 & 16 & 93 \\
\hline 28 & 2,040 & 15 & 83 & 1,500 & 6 & 24 & 2,180 & 17 & 100 \\
\hline 29 & 2,030 & 17 & 93 & -- & -- & -- & 2,210 & 18 & 107 \\
\hline 30 & 2,050 & 21 & 116 & -- & -- & -- & 2,300 & 26 & 161 \\
\hline 31 & 1,690 & 17 & 78 & -- & -- & -- & 2,800 & 73 & 552 \\
\hline TOTAL & 60,610 & -- & 11,207 & 48,380 & -- & $4,086.2$ & 60,490 & -- & 2,946 \\
\hline MEAN & 1,955 & 75 & 362 & 1,728 & 29 & 146 & 1,951 & 17 & 95 \\
\hline MAX & 3,850 & 221 & 1,370 & 2,560 & 98 & 503 & 2,800 & 73 & 552 \\
\hline MIN & 1,100 & 9 & 53 & 1,130 & 3 & 9.2 & 1,570 & 6 & 26 \\
\hline
\end{tabular}


Table 8. Daily mean streamflow and suspended-sediment data for Clark Fork above Missoula, Montana, October 2010 through September 2011.-Continued

[Abbreviations: acre-ft, acre-feet; $\mathrm{ft}^{3} / \mathrm{s}$, cubic feet per second; e, estimated; max, maximum; $\mathrm{mg} / \mathrm{L}$, milligrams per liter; min, minimum; ton/d, tons per day. Symbol: --, no data or value not computed]

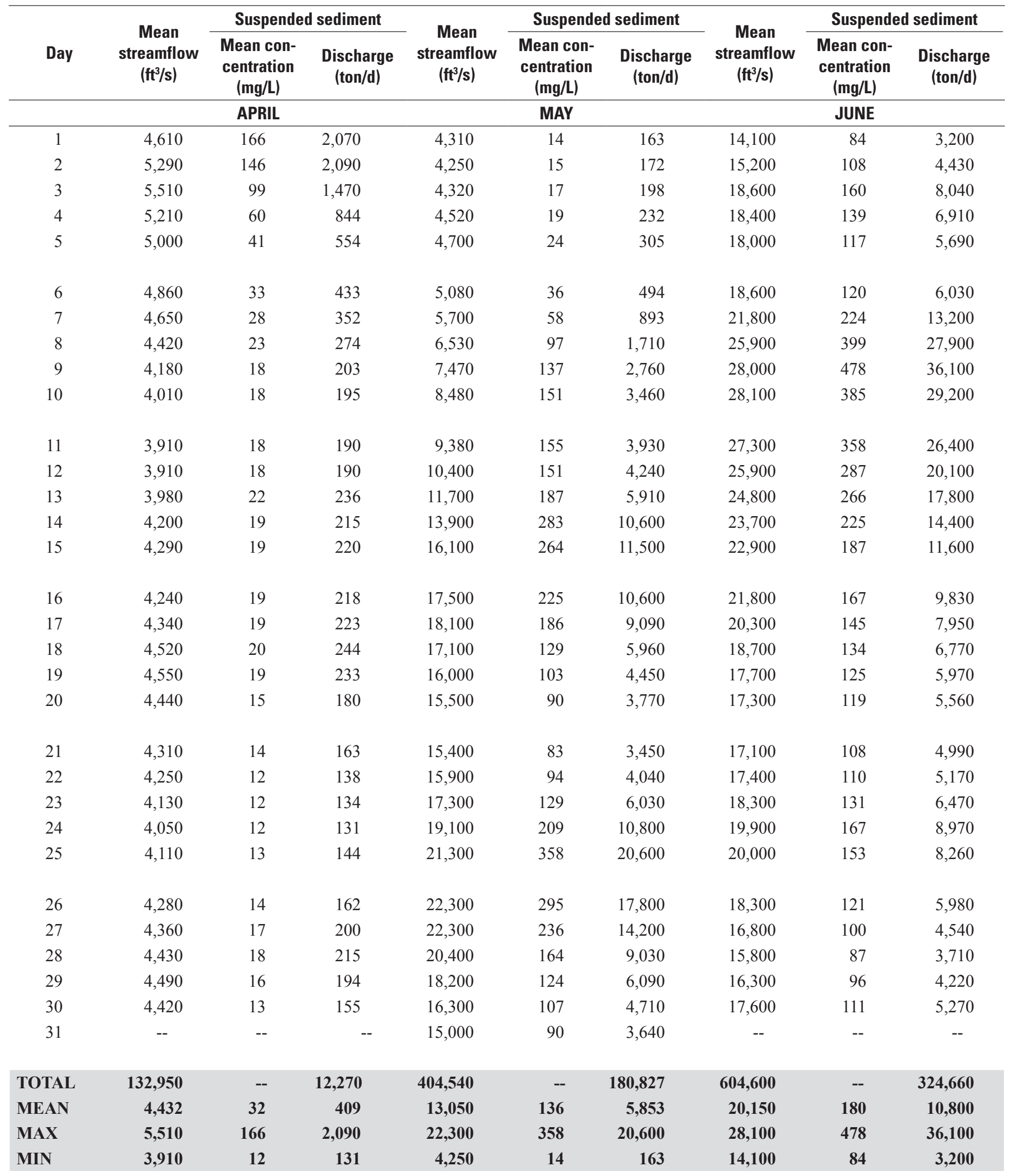


Table 8. Daily mean streamflow and suspended-sediment data for Clark Fork above Missoula, Montana, October 2010 through September 2011.-Continued

[Abbreviations: acre-ft, acre-feet; $\mathrm{ft}^{3} / \mathrm{s}$, cubic feet per second; e, estimated; max, maximum; $\mathrm{mg} / \mathrm{L}$, milligrams per liter; min, minimum; ton/d, tons per day. Symbol: --, no data or value not computed]

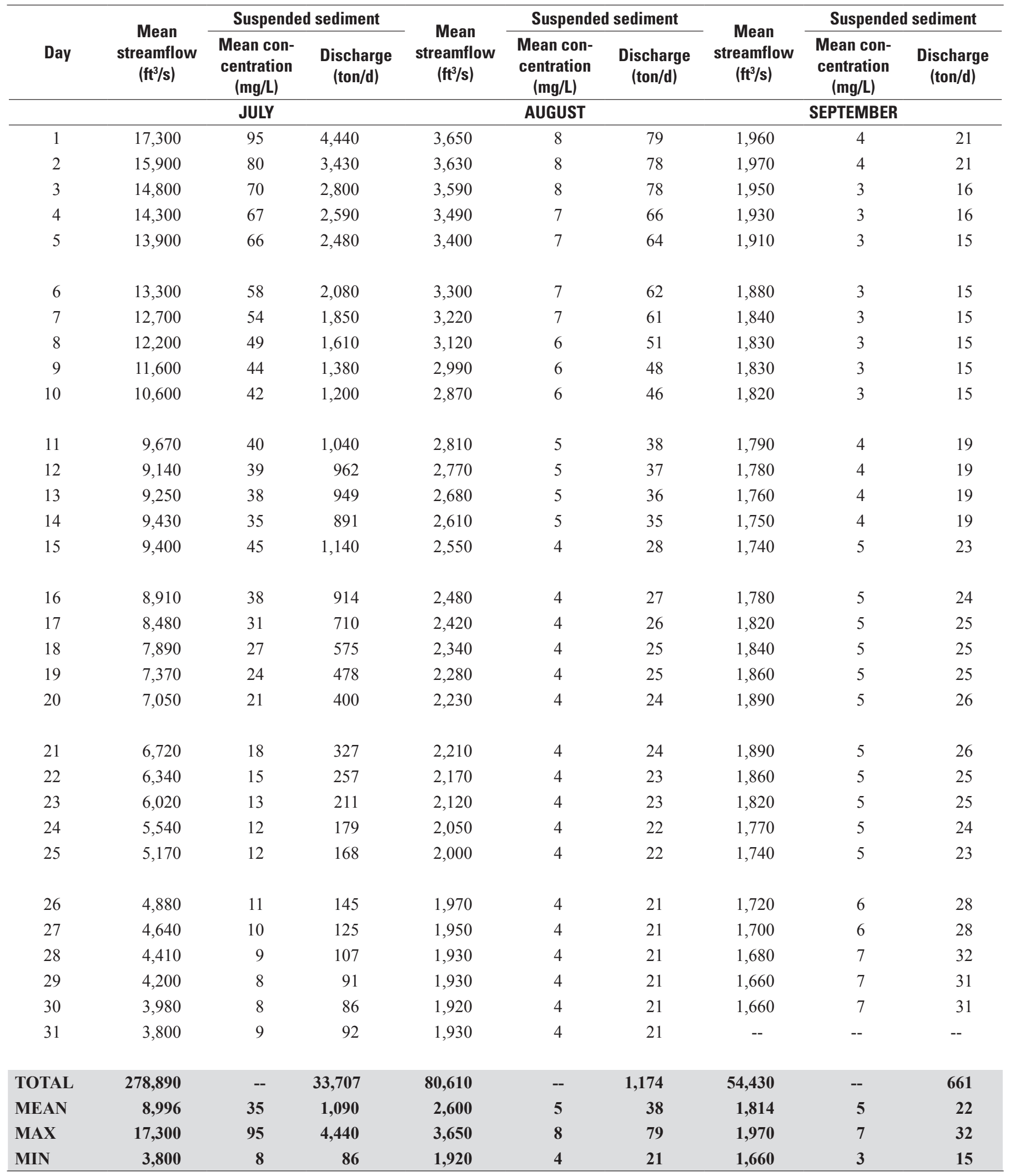

Total for water year 2011 (unrounded sum of daily values): streamflow-1,861,086 ft $3 / \mathrm{s}$ (annual runoff-3,691,000acre- $\mathrm{ft}$ ); suspended-sediment discharge- $577,279.2$ tons. 
Table 9. Seasonal daily maximum, minimum, and mean turbidity, with monthly summary statistics at Mill Creek near Anaconda, Montana, April through September 2011.

[Turbidity values are based on near-infrared monochrome light emitted at wavelengths of 780 to 900 nanometers with a detection angle of $90+/-2.5$ degrees to incident beam, reported in formazin nephelometric units (FNU). Symbol: --, no data]

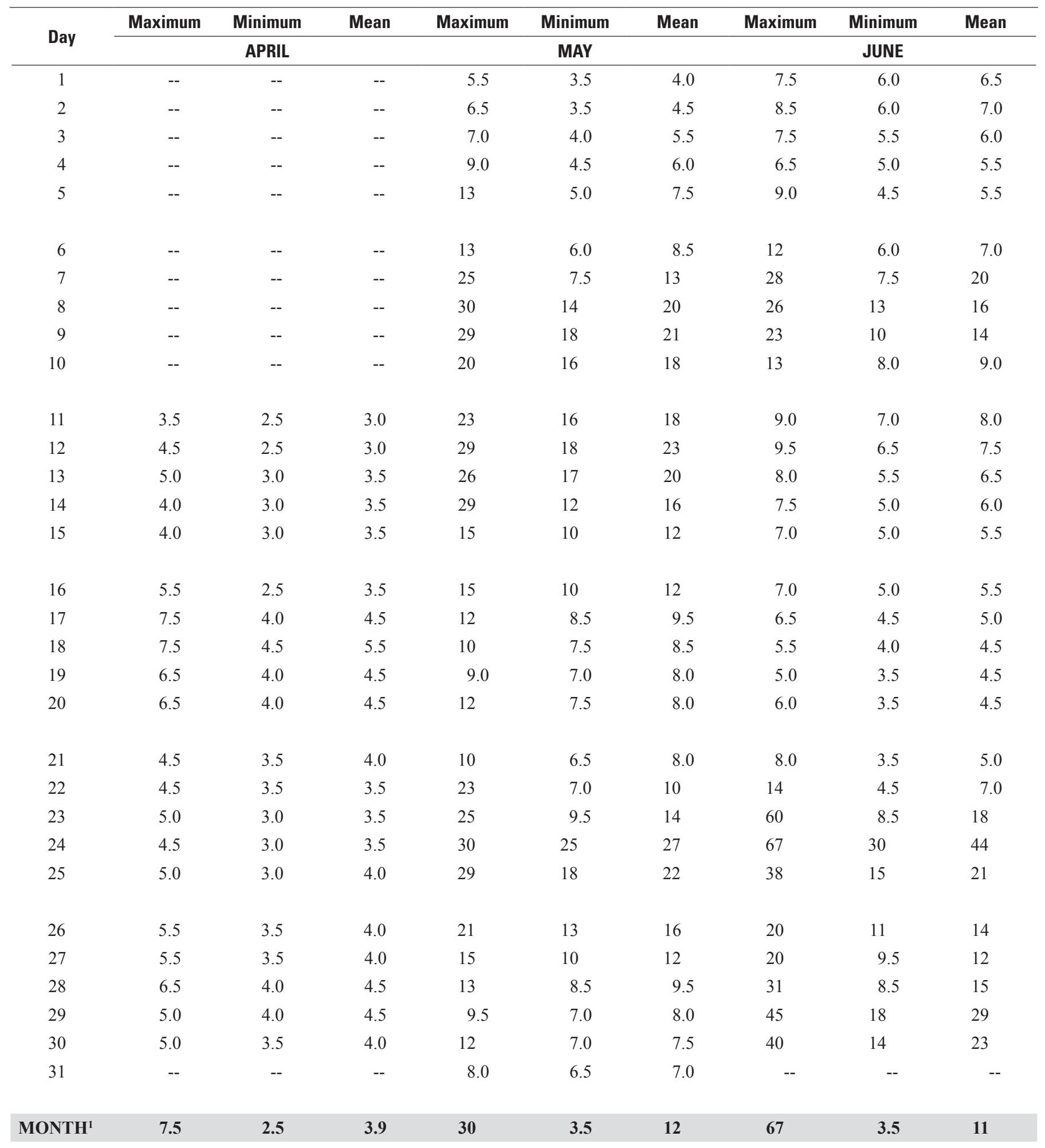


Table 9. Seasonal daily maximum, minimum, and mean turbidity, with monthly summary statistics at Mill Creek near Anaconda, Montana, April through September 2011.—Continued

[Turbidity values are based on near-infrared monochrome light emitted at wavelengths of 780 to 900 nanometers with a detection angle of $90+/-2.5$ degrees to incident beam, reported in formazin nephelometric units (FNU). Symbol: --, no data]

\begin{tabular}{|c|c|c|c|c|c|c|c|c|c|}
\hline \multirow{2}{*}{ Day } & Maximum & Minimum & Mean & Maximum & Minimum & Mean & Maximum & Minimum & Mean \\
\hline & \multicolumn{3}{|c|}{ JULY } & \multicolumn{3}{|c|}{ AUGUST } & \multicolumn{3}{|c|}{ SEPTEMBER } \\
\hline 1 & 17 & 9.0 & 12 & 5.0 & 1.5 & 2.5 & -- & -- & -- \\
\hline 2 & 12 & 7.0 & 8.5 & 3.5 & 1.5 & 2.0 & 2.5 & 0.5 & 1.5 \\
\hline 3 & 21 & 6.5 & 9.5 & 3.5 & 1.5 & 2.0 & 3.0 & .5 & 1.5 \\
\hline 4 & 26 & 8.0 & 13 & 3.5 & 1.5 & 2.0 & 3.0 & .5 & 1.0 \\
\hline 5 & 14 & 8.0 & 11 & 3.0 & 1.5 & 2.0 & 2.0 & .5 & 1.0 \\
\hline 6 & 14 & 7.0 & 10 & 3.0 & 1.0 & 2.0 & 2.0 & .5 & 1.0 \\
\hline 7 & 13 & 6.0 & 7.5 & 3.0 & 1.0 & 2.0 & 2.5 & .5 & 1.5 \\
\hline 8 & 12 & 6.0 & 7.5 & 3.5 & 1.0 & 2.0 & 2.0 & .5 & 1.0 \\
\hline 9 & 11 & 7.0 & 9.0 & 3.5 & 1.0 & 2.0 & 2.0 & .5 & 1.0 \\
\hline 10 & 7.5 & 5.5 & 6.5 & 3.5 & 1.0 & 2.0 & 2.5 & .5 & 1.0 \\
\hline 11 & 8.5 & 4.5 & 6.0 & 4.0 & 1.0 & 2.0 & 2.0 & .5 & 1.0 \\
\hline 12 & 9.5 & 4.5 & 6.0 & 3.0 & 1.0 & 2.0 & 2.0 & .5 & 1.0 \\
\hline 13 & 16 & 4.5 & 7.5 & 3.5 & 1.0 & 1.5 & 2.5 & .5 & 1.0 \\
\hline 14 & 16 & 6.0 & 10 & 3.0 & 1.0 & 2.0 & 2.5 & .5 & 1.5 \\
\hline 15 & 7.0 & 4.5 & 5.5 & 3.0 & 1.0 & 2.0 & 2.0 & .5 & 1.0 \\
\hline 16 & 6.0 & 4.0 & 4.5 & 3.5 & 1.0 & 2.0 & 3.0 & 1.0 & 1.5 \\
\hline 17 & 5.5 & 4.0 & 4.5 & 3.0 & 1.0 & 2.0 & 2.0 & .5 & 1.5 \\
\hline 18 & 6.0 & 3.5 & 4.5 & 3.5 & 1.0 & 2.0 & 2.0 & .5 & 1.0 \\
\hline 19 & 6.0 & 3.0 & 4.0 & 3.5 & 1.0 & 2.0 & 2.0 & .5 & 1.0 \\
\hline 20 & 5.0 & 3.0 & 4.0 & 3.0 & 1.0 & 2.0 & 3.5 & .5 & 1.5 \\
\hline 21 & 5.5 & 3.0 & 4.0 & 3.5 & 1.0 & 1.5 & 2.5 & .5 & 1.5 \\
\hline 22 & 5.5 & 2.5 & 3.5 & 3.5 & .5 & 1.5 & 3.0 & .5 & 1.5 \\
\hline 23 & 5.0 & 2.5 & 3.5 & 3.5 & .5 & 1.5 & 2.5 & .5 & 1.5 \\
\hline 24 & 5.0 & 2.5 & 3.5 & 3.0 & .5 & 1.5 & 2.5 & .5 & 1.5 \\
\hline 25 & 4.5 & 2.0 & 3.0 & 2.5 & 1.0 & 1.5 & 2.5 & .5 & 1.0 \\
\hline 26 & 4.0 & 2.0 & 3.0 & 2.5 & .5 & 1.5 & 2.0 & .5 & 1.0 \\
\hline 27 & 4.0 & 2.0 & 2.5 & 2.5 & .5 & 1.0 & 2.5 & .5 & 1.0 \\
\hline 28 & 4.0 & 2.0 & 2.5 & 2.0 & .5 & 1.0 & 3.0 & 1.0 & 1.5 \\
\hline 29 & 3.5 & 1.5 & 2.5 & 2.0 & 1.0 & 1.5 & -- & -- & -- \\
\hline 30 & 3.5 & 1.5 & 2.5 & 2.5 & .5 & 1.0 & -- & -- & -- \\
\hline 31 & 3.5 & 1.5 & 2.5 & 3.0 & .5 & 1.5 & -- & -- & -- \\
\hline MONTH $^{1}$ & 26 & 1.5 & 5.9 & 5.0 & .5 & 1.8 & 3.5 & .5 & 1.2 \\
\hline
\end{tabular}

${ }^{1}$ For months with missing daily values, the means are calculated using available values. 
Table 10. Seasonal daily maximum, minimum, and mean turbidity, with monthly summary statistics at Willow Creek near Anaconda, Montana, May through September 2011.

[Turbidity values are based on near-infrared monochrome light emitted at wavelengths of 780 to 900 nanometers with a detection angle of $90+/-2.5$ degrees to incident beam, reported in formazin nephelometric units (FNU). Symbols: --, no data]

\begin{tabular}{|c|c|c|c|c|c|c|c|c|c|}
\hline \multirow{2}{*}{ Day } & Maximum & Minimum & Mean & \multirow[t]{2}{*}{ Maximum } & \multirow{2}{*}{$\begin{array}{c}\text { Minimum } \\
\text { JUNE }\end{array}$} & Mean & Maximum & Minimum & Mean \\
\hline & & MAY & & & & \multicolumn{4}{|c|}{ JULY } \\
\hline 1 & -- & -- & -- & 30 & 14 & 17 & 8.0 & 5.0 & 6.0 \\
\hline 2 & -- & -- & -- & 29 & 20 & 22 & 8.0 & 5.0 & 6.0 \\
\hline 3 & -- & -- & -- & 26 & 18 & 21 & 8.0 & 5.0 & 6.0 \\
\hline 4 & -- & -- & -- & 29 & 16 & 20 & 7.0 & 4.5 & 5.5 \\
\hline 5 & -- & -- & -- & -- & -- & -- & 7.5 & 4.5 & 5.5 \\
\hline 6 & 28 & 17 & 21 & 110 & 8.0 & 46 & 7.0 & 4.5 & 5.5 \\
\hline 7 & 44 & 18 & 26 & 120 & 32 & 67 & 7.5 & 4.5 & 5.5 \\
\hline 8 & 64 & 22 & 31 & 45 & 18 & 26 & 7.0 & 4.0 & 5.0 \\
\hline 9 & 64 & 28 & 35 & 42 & 30 & 37 & 6.5 & 4.0 & 4.5 \\
\hline 10 & 38 & 25 & 30 & 110 & 9.0 & 40 & 6.0 & 4.0 & 4.5 \\
\hline 11 & 48 & 21 & 27 & 110 & 18 & 48 & 7.5 & 3.5 & 5.0 \\
\hline 12 & 56 & 27 & 38 & 39 & 23 & 31 & 7.5 & 3.5 & 4.5 \\
\hline 13 & 90 & 35 & 49 & 36 & 23 & 28 & -- & -- & -- \\
\hline 14 & 67 & 33 & 48 & 37 & 21 & 25 & -- & -- & -- \\
\hline 15 & 37 & 26 & 30 & 41 & 17 & 22 & -- & -- & -- \\
\hline 16 & 33 & 24 & 27 & 23 & 14 & 17 & -- & -- & -- \\
\hline 17 & 32 & 18 & 21 & 20 & 13 & 15 & -- & -- & -- \\
\hline 18 & 19 & 16 & 17 & 17 & 11 & 13 & -- & -- & -- \\
\hline 19 & 17 & 15 & 16 & 23 & 11 & 13 & -- & -- & -- \\
\hline 20 & 19 & 14 & 15 & 13 & 9.5 & 11 & 8.0 & 5.5 & 6.0 \\
\hline 21 & 16 & 14 & 15 & 13 & 9.5 & 10 & 10 & 5.0 & 6.5 \\
\hline 22 & 57 & 14 & 18 & 10 & 8.0 & 9.0 & 11 & 5.0 & 6.5 \\
\hline 23 & 62 & 29 & 42 & 11 & 8.0 & 8.5 & 8.0 & 5.0 & 6.0 \\
\hline 24 & 290 & 62 & 150 & 9.5 & 7.0 & 8.0 & 32 & 6.0 & 9.0 \\
\hline 25 & 300 & 62 & 170 & 10 & 7.0 & 8.0 & 14 & 5.5 & 7.0 \\
\hline 26 & 87 & 33 & 65 & 9.5 & 7.0 & 7.5 & 11 & 5.5 & 7.0 \\
\hline 27 & 59 & 27 & 38 & 8.5 & 6.5 & 7.5 & 14 & 5.0 & 7.0 \\
\hline 28 & 35 & 22 & 27 & 8.5 & 6.0 & 7.0 & 9.5 & 5.0 & 6.5 \\
\hline 29 & 46 & 17 & 26 & 8.5 & 5.5 & 6.5 & 11 & 4.5 & 6.5 \\
\hline 30 & 25 & 16 & 19 & 8.5 & 5.5 & 6.0 & 11 & 4.5 & 6.0 \\
\hline 31 & 20 & 15 & 16 & -- & -- & -- & 10 & 4.5 & 6.5 \\
\hline MONTH $^{1}$ & 300 & 14 & 39 & 120 & 5.5 & 21 & 32 & 3.5 & 6.0 \\
\hline
\end{tabular}


Table 10. Seasonal daily maximum, minimum, and mean turbidity, with monthly summary statistics at Willow Creek near Anaconda, Montana, May through September 2011.-Continued

[Turbidity values are based on near-infrared monochrome light emitted at wavelengths of 780 to 900 nanometers with a detection angle of $90+/-2.5$ degrees to incident beam, reported in formazin nephelometric units (FNU). Symbols: --, no data]

\begin{tabular}{|c|c|c|c|c|c|c|}
\hline \multirow{2}{*}{ Day } & Maximum & Minimum & Mean & Maximum & Minimum & Mean \\
\hline & \multicolumn{3}{|c|}{ AUGUST } & \multicolumn{3}{|c|}{ SEPTEMBER } \\
\hline 1 & 10 & 4.0 & 6.0 & 8.0 & 3.5 & 4.5 \\
\hline 2 & 9.5 & 4.0 & 6.0 & 7.5 & 3.5 & 4.5 \\
\hline 3 & 10 & 4.0 & 6.0 & 8.0 & 3.0 & 4.5 \\
\hline 4 & 9.5 & 4.0 & 5.5 & 7.0 & 3.0 & 4.5 \\
\hline 5 & 9.5 & 4.0 & 6.0 & 7.0 & 3.5 & 5.0 \\
\hline 6 & 7.5 & 4.0 & 5.0 & 6.5 & 3.0 & 4.5 \\
\hline 7 & 8.5 & 4.0 & 5.0 & 7.0 & 3.5 & 5.0 \\
\hline 8 & 8.5 & 4.0 & 5.0 & 7.0 & 3.5 & 4.5 \\
\hline 9 & 8.5 & 3.5 & 5.0 & 7.5 & 3.0 & 4.5 \\
\hline 10 & 9.5 & 3.5 & 5.0 & 7.5 & 3.0 & 4.5 \\
\hline 11 & 8.5 & 3.5 & 5.0 & 6.5 & 3.0 & 4.0 \\
\hline 12 & 8.5 & 3.5 & 5.0 & 8.5 & 3.0 & 4.5 \\
\hline 13 & 8.5 & 3.5 & 5.0 & 8.5 & 2.5 & 4.0 \\
\hline 14 & 7.0 & 3.0 & 4.5 & 6.5 & 2.5 & 4.0 \\
\hline 15 & 9.0 & 3.0 & 4.5 & 13 & 3.5 & 5.5 \\
\hline 16 & 8.0 & 3.0 & 4.5 & 17 & 3.5 & 6.0 \\
\hline 17 & 9.5 & 3.0 & 5.5 & 11 & 4.0 & 6.0 \\
\hline 18 & 9.0 & 4.0 & 5.5 & 13 & 5.0 & 8.5 \\
\hline 19 & 9.0 & 4.0 & 5.5 & 22 & 7.0 & 13 \\
\hline 20 & 9.0 & 4.5 & 6.0 & -- & -- & -- \\
\hline 21 & 10 & 4.0 & 5.5 & -- & -- & -- \\
\hline 22 & 10 & 4.0 & 6.0 & -- & -- & -- \\
\hline 23 & 8.0 & 4.0 & 5.0 & -- & -- & -- \\
\hline 24 & 11 & 4.0 & 5.5 & -- & -- & -- \\
\hline 25 & 9.0 & 4.5 & 6.5 & -- & -- & -- \\
\hline 26 & 6.0 & 4.0 & 5.0 & -- & -- & -- \\
\hline 27 & 8.5 & 4.0 & 5.5 & -- & -- & -- \\
\hline 28 & 8.5 & 3.5 & 5.0 & -- & -- & -- \\
\hline 29 & 7.0 & 3.5 & 4.5 & -- & -- & -- \\
\hline 30 & 8.0 & 3.5 & 5.0 & -- & -- & -- \\
\hline 31 & 9.5 & 3.5 & 5.0 & -- & -- & -- \\
\hline MONTH $^{1}$ & 11 & 3.0 & 5.3 & 22 & 2.5 & 5.3 \\
\hline
\end{tabular}

${ }^{1}$ For months with missing daily values, the means are calculated using available values. 
Table 11. Seasonal daily maximum, minimum, and mean turbidity, with monthly summary statistics at Warm Springs Creek near Anaconda, Montana, April through September 2011.

[Turbidity values are based on near-infrared monochrome light emitted at wavelengths of 780 to 900 nanometers with a detection angle of $90+/-2.5$ degrees to incident beam, reported in formazin nephelometric units (FNU). Symbols: --, no data]

\begin{tabular}{|c|c|c|c|c|c|c|c|c|c|}
\hline \multirow{2}{*}{ Day } & Maximum & Minimum & Mean & Maximum & Minimum & Mean & Maximum & Minimum & Mean \\
\hline & \multicolumn{3}{|c|}{ APRIL } & \multicolumn{3}{|c|}{ MAY } & \multicolumn{3}{|c|}{ JUNE } \\
\hline 1 & -- & -- & -- & 5.0 & 2.5 & 3.0 & 5.0 & 3.5 & 4.5 \\
\hline 2 & -- & -- & -- & 3.5 & 3.0 & 3.0 & 26 & 4.0 & 6.0 \\
\hline 3 & -- & -- & -- & 4.0 & 2.5 & 3.0 & 5.0 & 3.5 & 4.0 \\
\hline 4 & -- & -- & -- & 4.0 & 2.5 & 3.0 & 4.5 & 3.0 & 4.0 \\
\hline 5 & -- & -- & -- & 6.5 & 2.5 & 3.5 & 5.0 & 3.5 & 4.0 \\
\hline 6 & -- & -- & -- & 5.0 & 3.0 & 3.5 & 8.0 & 4.5 & 5.5 \\
\hline 7 & -- & -- & -- & 5.5 & 3.0 & 4.0 & 54 & 8.0 & 29 \\
\hline 8 & -- & -- & -- & 10 & 3.5 & 5.0 & 54 & 18 & 30 \\
\hline 9 & -- & -- & -- & 20 & 5.5 & 8.5 & 20 & 11 & 15 \\
\hline 10 & -- & -- & -- & 14 & 4.0 & 5.5 & 16 & 9.0 & 11 \\
\hline 11 & 4.0 & 2.5 & 3.0 & 7.5 & 4.0 & 5.0 & 16 & 8.0 & 9.5 \\
\hline 12 & 3.5 & 2.5 & 3.0 & 6.5 & 4.0 & 5.0 & 9.5 & 6.0 & 7.0 \\
\hline 13 & 32 & 3.0 & 4.0 & 16 & 5.0 & 7.0 & 7.5 & 5.0 & 6.0 \\
\hline 14 & 5.0 & 3.0 & 3.0 & 18 & 7.0 & 11 & 6.5 & 5.0 & 6.0 \\
\hline 15 & 3.5 & 2.5 & 3.0 & 18 & 6.5 & 10 & 9.0 & 5.5 & 6.5 \\
\hline 16 & 4.5 & 2.5 & 3.0 & 21 & 5.5 & 12 & 6.5 & 4.5 & 5.5 \\
\hline 17 & 4.5 & 3.0 & 3.0 & 11 & 5.5 & 7.0 & 5.5 & 4.5 & 5.0 \\
\hline 18 & 3.5 & 3.0 & 3.0 & 6.0 & 4.5 & 5.0 & 6.0 & 4.0 & 4.5 \\
\hline 19 & 4.0 & 2.5 & 3.0 & 6.0 & 4.0 & 5.0 & 5.5 & 4.0 & 4.5 \\
\hline 20 & 8.0 & 2.5 & 3.5 & 6.5 & 4.5 & 5.5 & 6.5 & 4.0 & 5.0 \\
\hline 21 & 6.0 & 3.0 & 3.5 & 7.0 & 4.5 & 5.5 & 8.5 & 5.0 & 6.0 \\
\hline 22 & 4.0 & 2.5 & 3.0 & 10 & 5.5 & 6.5 & 15 & 8.0 & 10 \\
\hline 23 & 5.0 & 2.5 & 3.0 & -- & -- & -- & 30 & 11 & 17 \\
\hline 24 & 4.0 & 2.5 & 3.0 & -- & -- & -- & 39 & 16 & 28 \\
\hline 25 & 4.0 & 2.5 & 3.0 & -- & -- & -- & 19 & 9.5 & 14 \\
\hline 26 & 5.0 & 2.5 & 3.0 & -- & -- & -- & 12 & 6.5 & 8.5 \\
\hline 27 & 5.5 & 2.5 & 3.0 & 10 & 6.5 & 7.5 & 9.5 & 5.5 & 6.5 \\
\hline 28 & 3.5 & 3.0 & 3.0 & 8.0 & 5.0 & 6.0 & 16 & 5.5 & 7.0 \\
\hline 29 & 3.5 & 3.0 & 3.0 & 9.0 & 4.5 & 5.0 & 18 & 9.5 & 13 \\
\hline 30 & 3.5 & 2.5 & 3.0 & 26 & 4.5 & 6.0 & 14 & 9.5 & 12 \\
\hline 31 & -- & -- & -- & 5.5 & 4.0 & 4.5 & -- & -- & -- \\
\hline MONTH $^{1}$ & 32 & 2.5 & 3.1 & 26 & 2.5 & 5.8 & 54 & 3.0 & 9.8 \\
\hline
\end{tabular}


Table 11. Seasonal daily maximum, minimum, and mean turbidity, with monthly summary statistics at Warm Springs Creek near Anaconda, Montana, April through September 2011.-Continued

[Turbidity values are based on near-infrared monochrome light emitted at wavelengths of 780 to 900 nanometers with a detection angle of $90+/-2.5$ degrees to incident beam, reported in formazin nephelometric units (FNU). Symbols: --, no data]

\begin{tabular}{|c|c|c|c|c|c|c|c|c|c|}
\hline \multirow{2}{*}{ Day } & Maximum & Minimum & Mean & Maximum & Minimum & Mean & Maximum & Minimum & Mean \\
\hline & \multicolumn{3}{|c|}{ JULY } & \multicolumn{3}{|c|}{ AUGUST } & \multicolumn{3}{|c|}{ SEPTEMBER } \\
\hline 1 & 12 & 7.0 & 9.5 & 3.0 & 2.5 & 2.5 & 4.0 & 2.5 & 2.5 \\
\hline 2 & 12 & 6.5 & 7.5 & 2.5 & 2.0 & 2.5 & 4.0 & 2.5 & 2.5 \\
\hline 3 & 10 & 5.5 & 7.5 & 4.0 & 2.0 & 2.5 & 4.5 & 2.5 & 2.5 \\
\hline 4 & 13 & 6.5 & 9.0 & 3.0 & 2.0 & 2.5 & 3.5 & 2.5 & 2.5 \\
\hline 5 & 16 & 5.5 & 8.5 & 3.0 & 2.0 & 2.5 & 5.0 & 2.0 & 2.5 \\
\hline 6 & 24 & 5.0 & 8.0 & 4.0 & 2.0 & 2.5 & 3.0 & 2.0 & 2.5 \\
\hline 7 & 8.0 & 5.0 & 6.0 & 3.0 & 2.0 & 2.5 & 3.5 & 2.0 & 2.5 \\
\hline 8 & 7.0 & 4.5 & 5.5 & 3.0 & 2.0 & 2.5 & 4.5 & 2.5 & 2.5 \\
\hline 9 & 8.5 & 5.0 & 6.0 & 3.0 & 2.0 & 2.5 & 3.0 & 2.0 & 2.5 \\
\hline 10 & 11 & 4.5 & 5.5 & 3.0 & 2.0 & 2.5 & 4.0 & 2.5 & 2.5 \\
\hline 11 & 9.5 & 3.5 & 4.5 & 3.0 & 2.5 & 2.5 & 3.0 & 2.5 & 3.0 \\
\hline 12 & 6.0 & 3.5 & 4.5 & 3.0 & 2.0 & 2.5 & 3.5 & 2.5 & 2.5 \\
\hline 13 & 7.0 & 4.0 & 4.5 & 3.0 & 2.0 & 2.5 & 3.5 & 2.0 & 2.5 \\
\hline 14 & 6.5 & 4.0 & 5.0 & 3.0 & 2.0 & 2.5 & 3.0 & 2.0 & 2.5 \\
\hline 15 & 5.0 & 3.5 & 4.0 & 3.0 & 2.0 & 2.5 & 4.5 & 2.0 & 2.5 \\
\hline 16 & 4.5 & 3.0 & 3.5 & 3.0 & 2.0 & 2.5 & 3.5 & 2.5 & 3.0 \\
\hline 17 & 5.0 & 3.0 & 3.5 & 3.0 & 2.5 & 2.5 & 3.5 & 2.5 & 2.5 \\
\hline 18 & 4.0 & 3.0 & 3.5 & -- & -- & -- & 3.0 & 2.0 & 2.5 \\
\hline 19 & 5.0 & 3.0 & 3.5 & 3.5 & 2.5 & 2.5 & 3.0 & 2.5 & 2.5 \\
\hline 20 & 4.5 & 3.0 & 3.0 & 3.5 & 2.5 & 3.0 & 3.0 & 2.0 & 2.5 \\
\hline 21 & 4.0 & 3.0 & 3.0 & 3.0 & 2.5 & 2.5 & 3.0 & 2.0 & 2.5 \\
\hline 22 & 4.0 & 2.5 & 3.0 & 3.5 & 2.5 & 3.0 & 3.0 & 2.0 & 2.5 \\
\hline 23 & 4.0 & 2.5 & 3.0 & 4.0 & 2.5 & 2.5 & 3.5 & 2.0 & 2.5 \\
\hline 24 & 4.5 & 2.5 & 3.0 & 4.0 & 2.5 & 2.5 & 3.0 & 2.0 & 2.5 \\
\hline 25 & 3.5 & 2.5 & 3.0 & 4.5 & 2.5 & 3.0 & 3.0 & 2.0 & 2.5 \\
\hline 26 & 3.5 & 2.5 & 3.0 & 3.5 & 2.5 & 3.0 & 3.0 & 2.0 & 2.5 \\
\hline 27 & 4.0 & 2.5 & 2.5 & 3.5 & 2.5 & 3.0 & 3.0 & 2.0 & 2.5 \\
\hline 28 & 3.5 & 2.5 & 2.5 & 3.5 & 2.5 & 3.0 & 3.0 & 2.0 & 2.5 \\
\hline 29 & 3.5 & 2.5 & 3.0 & 81 & 2.5 & 7.0 & 3.0 & 2.0 & 2.5 \\
\hline 30 & 3.5 & 2.5 & 2.5 & 3.5 & 2.5 & 3.0 & 2.5 & 2.0 & 2.5 \\
\hline 31 & 5.0 & 2.5 & 2.5 & 3.5 & 2.5 & 3.0 & -- & -- & -- \\
\hline MONTH $^{1}$ & 24 & 2.5 & 4.6 & 81 & 2.0 & 2.8 & 5.0 & 2.0 & 2.5 \\
\hline
\end{tabular}

${ }^{1}$ For months with missing daily values, the means are calculated using available values. 
Table 12. Seasonal daily maximum, minimum, and mean turbidity, with monthly summary statistics at Lost Creek near Anaconda, Montana, April through September 2011.

[Turbidity values are based on near-infrared monochrome light emitted at wavelengths of 780 to 900 nanometers with a detection angle of $90+/-2.5$ degrees to incident beam, reported in formazin nephelometric units (FNU). Symbols: $<$, less than; --, no data]

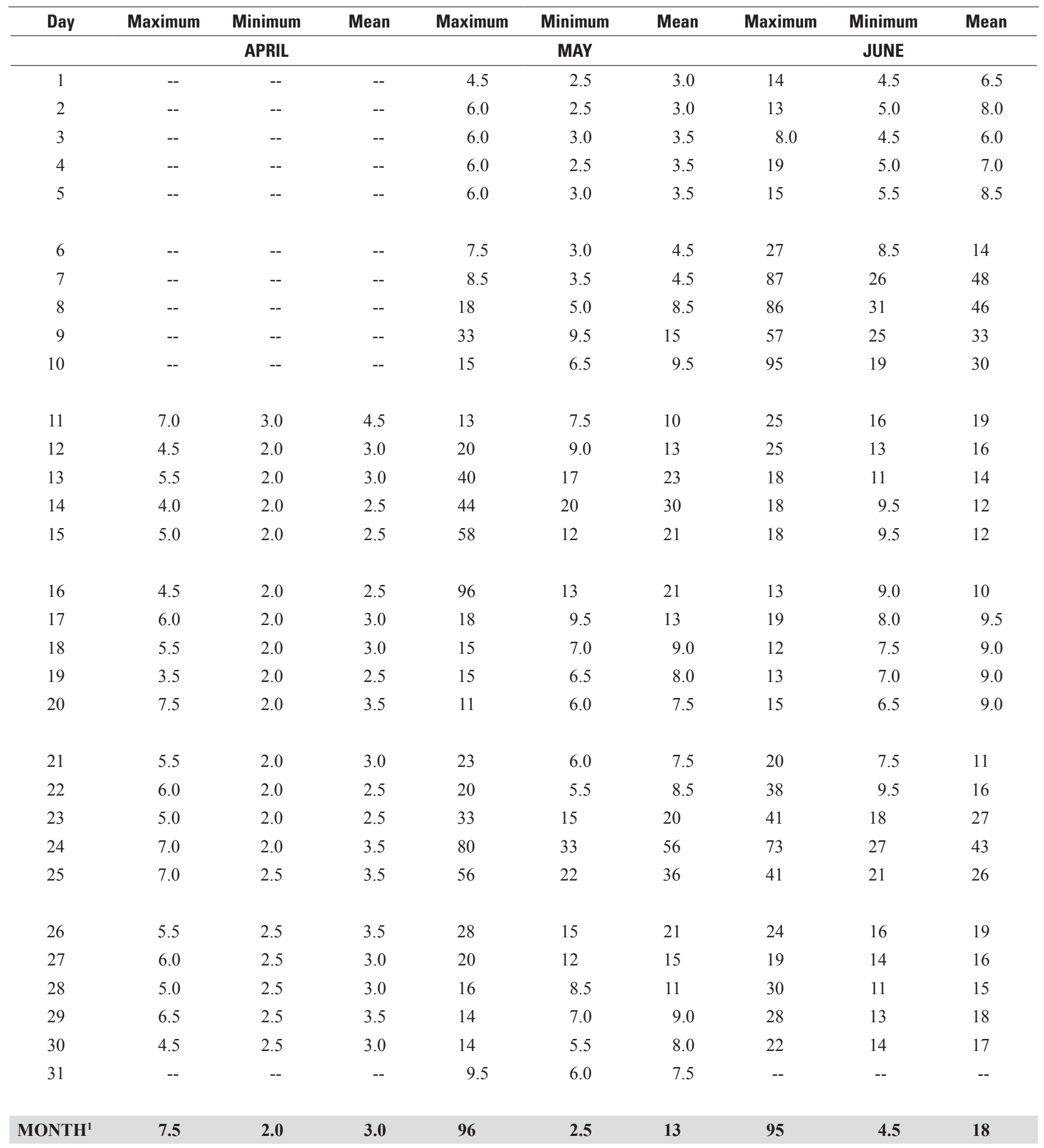


Table 12. Seasonal daily maximum, minimum, and mean turbidity, with monthly summary statistics at Lost Creek near Anaconda, Montana, April through September 2011.—Continued

[Turbidity values are based on near-infrared monochrome light emitted at wavelengths of 780 to 900 nanometers with a detection angle of $90+/-2.5$ degrees to incident beam, reported in formazin nephelometric units (FNU). Symbols: $<$, less than; --, no data]

\begin{tabular}{|c|c|c|c|c|c|c|c|c|c|}
\hline \multirow{2}{*}{ Day } & Maximum & Minimum & Mean & Maximum & Minimum & Mean & Maximum & Minimum & Mean \\
\hline & \multicolumn{3}{|c|}{ JULY } & \multicolumn{3}{|c|}{ AUGUST } & \multicolumn{3}{|c|}{ SEPTEMBER } \\
\hline 1 & 20 & 12 & 15 & 5.5 & 2.5 & 3.5 & 5.0 & 1.5 & 2.0 \\
\hline 2 & 16 & 9.5 & 12 & 6.5 & 3.0 & 3.5 & 3.0 & 1.5 & 2.0 \\
\hline 3 & 16 & 8.5 & 11 & 14 & 3.0 & 5.0 & 2.5 & 1.5 & 2.0 \\
\hline 4 & 15 & 8.0 & 10 & 10 & 3.5 & 5.5 & 2.0 & 1.5 & 1.5 \\
\hline 5 & 12 & 7.0 & 9.0 & 6.5 & 3.0 & 4.0 & 2.0 & 1.5 & 1.5 \\
\hline 6 & 12 & 7.0 & 8.5 & 7.0 & 3.0 & 4.0 & 2.0 & 1.5 & 1.5 \\
\hline 7 & 16 & 7.0 & 9.0 & 8.5 & 3.0 & 4.0 & 2.0 & 1.5 & 1.5 \\
\hline 8 & 17 & 7.0 & 9.0 & 6.5 & 2.0 & 3.5 & 2.0 & 1.5 & 1.5 \\
\hline 9 & 11 & 6.5 & 8.5 & 6.5 & 2.5 & 4.0 & 2.0 & 1.5 & 1.5 \\
\hline 10 & 23 & 6.0 & 8.5 & 6.5 & 2.0 & 3.5 & 2.0 & 1.5 & 1.5 \\
\hline 11 & 10 & 6.0 & 7.5 & 6.5 & 2.0 & 3.5 & 2.0 & 1.5 & 1.5 \\
\hline 12 & 14 & 5.5 & 7.0 & 6.5 & 2.0 & 3.0 & 2.0 & 1.5 & 1.5 \\
\hline 13 & 150 & 5.0 & 13 & 7.5 & 2.0 & 3.0 & 2.5 & 1.5 & 1.5 \\
\hline 14 & 15 & 5.0 & 7.0 & 5.0 & 2.5 & 3.5 & 2.0 & 1.5 & 1.5 \\
\hline 15 & 7.5 & 4.5 & 5.5 & 4.5 & 2.5 & 3.5 & 2.0 & 1.5 & 1.5 \\
\hline 16 & 8.0 & 4.5 & 5.5 & 5.5 & 2.5 & 3.0 & 2.0 & 1.5 & 1.5 \\
\hline 17 & 9.5 & 4.0 & 5.5 & 4.5 & 2.5 & 3.0 & 2.0 & 1.5 & 1.5 \\
\hline 18 & 15 & 4.0 & 5.5 & 4.5 & 2.0 & 3.0 & 1.5 & 1.5 & 1.5 \\
\hline 19 & 17 & 4.5 & 7.0 & 6.0 & 2.5 & 3.5 & 2.0 & 1.5 & 1.5 \\
\hline 20 & 6.5 & 4.0 & 5.0 & 13 & 2.5 & 4.5 & 2.0 & 1.5 & 1.5 \\
\hline 21 & 8.0 & 3.5 & 5.0 & 8.0 & 2.5 & 3.5 & 2.0 & 1.5 & 1.5 \\
\hline 22 & 9.0 & 3.5 & 5.0 & 5.5 & 2.0 & 2.5 & 2.0 & 1.5 & 1.5 \\
\hline 23 & 8.0 & 3.5 & 4.5 & 5.5 & 1.5 & 2.5 & 2.0 & 1.0 & 1.5 \\
\hline 24 & 12 & 3.5 & 5.5 & 5.0 & 2.0 & 2.5 & 2.0 & 1.0 & 1.5 \\
\hline 25 & 8.0 & 4.0 & 5.5 & 4.5 & 2.0 & 2.5 & 1.5 & 1.0 & 1.5 \\
\hline 26 & 11 & 4.0 & 6.0 & 4.0 & 2.0 & 2.5 & 2.0 & 1.0 & 1.5 \\
\hline 27 & 7.0 & 3.5 & 5.0 & 3.0 & 1.5 & 2.0 & 2.5 & 1.0 & 2.0 \\
\hline 28 & 11 & 3.5 & 6.0 & 3.0 & 1.5 & 2.0 & 2.5 & 1.0 & 1.5 \\
\hline 29 & 9.0 & 3.0 & 5.0 & 2.5 & 1.5 & 2.0 & 4.0 & 1.5 & 1.5 \\
\hline 30 & 7.5 & 3.0 & 4.0 & 4.0 & 1.5 & 2.0 & 4.5 & 1.5 & 1.5 \\
\hline 31 & 6.5 & 2.5 & 3.5 & 7.5 & 1.5 & 2.0 & -- & -- & -- \\
\hline MONTH $^{1}$ & 150 & 2.5 & 7.2 & 14 & 1.5 & 3.2 & 5.0 & 1.0 & 1.6 \\
\hline
\end{tabular}

${ }^{1}$ For months with missing daily values, the means are calculated using available values. 
Table 13. Analyses of field replicates for water samples, Clark Fork basin, Montana.

[Abbreviations: $\mathrm{CaCO}_{3}$, calcium carbonate; $\mu \mathrm{g} / \mathrm{L}$, micrograms per liter; $\mathrm{mg} / \mathrm{L}$, milligrams per liter; mm, millimeter. Symbol: <, less than laboratory reporting level]

\begin{tabular}{|c|c|c|c|c|c|c|c|c|c|}
\hline $\begin{array}{l}\text { Site number } \\
\text { (fig. 1) }\end{array}$ & Site name & Date & Time & $\begin{array}{c}\text { Hardness, } \\
\text { filtered } \\
\text { (mg/L as } \mathrm{CaCO}_{3} \text { ) }\end{array}$ & $\begin{array}{l}\text { Calcium, } \\
\text { filtered } \\
\text { (mg/L) }\end{array}$ & $\begin{array}{c}\text { Magnesium } \\
\text { filtered } \\
\text { (mg/L) }\end{array}$ & $\begin{array}{c}\text { Arsenic, } \\
\text { filtered } \\
\text { ( } \mu \mathrm{g} / \mathrm{L})\end{array}$ & $\begin{array}{c}\text { Arsenic, } \\
\text { unfiltered } \\
\text { recoverable } \\
(\mu \mathrm{g} / \mathrm{L})\end{array}$ & $\begin{array}{c}\text { Cadmium, } \\
\text { filtered } \\
\text { ( } \mu \mathrm{g} / \mathrm{L})\end{array}$ \\
\hline \multirow[t]{2}{*}{12323600} & \multirow[t]{2}{*}{ Silver Bow Creek at Opportunity } & $05 / 25 / 2011$ & 1030 & 99.7 & 29.6 & 6.26 & 14.9 & 46.7 & 0.74 \\
\hline & & $05 / 25 / 2011$ & 1040 & 98.1 & 29.1 & 6.16 & 14.5 & 47.6 & .74 \\
\hline \multirow[t]{2}{*}{12323700} & \multirow[t]{2}{*}{ Mill Creek at Opportunity } & $08 / 15 / 2011$ & 1520 & 64.5 & 17.8 & 4.85 & 19.3 & 19.8 & .05 \\
\hline & & $08 / 15 / 2011$ & 1525 & 66.3 & 18.3 & 5.00 & 19.6 & 19.6 & .04 \\
\hline \multirow[t]{2}{*}{12323750} & \multirow[t]{2}{*}{ Silver Bow Creek at Warm Springs } & 07/13/2011 & 1010 & 74.9 & 22.5 & 4.52 & 21.4 & 24.1 & .03 \\
\hline & & 07/13/2011 & 1015 & 76.7 & 23.1 & 4.65 & 22.4 & 24.3 & .04 \\
\hline \multirow[t]{2}{*}{12323800} & \multirow[t]{2}{*}{ Clark Fork near Galen } & 06/08/2011 & 1145 & 106 & 32.6 & 5.83 & 28.2 & 36.1 & .13 \\
\hline & & 06/08/2011 & 1155 & 106 & 32.9 & 5.85 & 28.2 & 35.7 & .13 \\
\hline \multirow[t]{2}{*}{12323840} & \multirow[t]{2}{*}{ Lost Creek near Anaconda } & $04 / 11 / 2011$ & 1510 & 111 & 32.6 & 7.09 & 2.6 & 3.3 & .02 \\
\hline & & $04 / 11 / 2011$ & 1515 & 115 & 33.8 & 7.32 & 2.3 & 3.3 & .02 \\
\hline \multirow[t]{4}{*}{12324200} & \multirow[t]{4}{*}{ Clark Fork at Deer Lodge } & 05/17/2011 & 1225 & 138 & 41.1 & 8.53 & 16.8 & 35.6 & .07 \\
\hline & & 05/17/2011 & 1230 & 146 & 43.1 & 9.37 & 16.9 & 35.5 & .08 \\
\hline & & $06 / 24 / 2011$ & 1130 & 115 & 34.9 & 6.69 & 32.9 & 37.8 & .22 \\
\hline & & $06 / 24 / 2011$ & 1135 & 115 & 35.1 & 6.73 & 32.4 & 37.0 & .22 \\
\hline \multirow[t]{2}{*}{12324400} & \multirow{2}{*}{$\begin{array}{l}\text { Clark Fork above Little Blackfoot, } \\
\text { near Garrison }\end{array}$} & 03/15/2011 & 1500 & 206 & 60.5 & 13.2 & 9.3 & 13.3 & .06 \\
\hline & & 03/15/2011 & 1505 & 213 & 62.4 & 13.9 & 9.3 & 13.6 & .07 \\
\hline
\end{tabular}


Table 13. Analyses of field replicates for water samples, Clark Fork basin, Montana.-Continued

[Abbreviations: $\mathrm{CaCO}_{3}$, calcium carbonate; $\mu \mathrm{g} / \mathrm{L}$, micrograms per liter; $\mathrm{mg} / \mathrm{L}$, milligrams per liter; mm, millimeter. Symbol: <, less than laboratory reporting level]

\begin{tabular}{|c|c|c|c|c|c|c|c|c|c|c|}
\hline $\begin{array}{l}\text { Site number } \\
\quad \text { (fig. 1) }\end{array}$ & Site name & Date & Time & $\begin{array}{c}\text { Cadmium, } \\
\text { unfiltered } \\
\text { recoverable } \\
(\mu \mathrm{g} / \mathrm{L})\end{array}$ & $\begin{array}{c}\text { Copper, } \\
\text { filtered } \\
\text { ( } \mu \mathrm{g} / \mathrm{L})\end{array}$ & $\begin{array}{c}\text { Copper, } \\
\text { unfiltered } \\
\text { recoverable } \\
(\mu \mathrm{g} / \mathrm{L})\end{array}$ & $\begin{array}{c}\text { Iron, } \\
\text { filtered } \\
(\mu \mathrm{g} / \mathrm{L})\end{array}$ & $\begin{array}{c}\text { Iron, } \\
\text { unfiltered } \\
\text { recoverable } \\
(\mu \mathrm{g} / \mathrm{L})\end{array}$ & $\begin{array}{c}\text { Lead, } \\
\text { filtered } \\
\text { ( } \mu \mathrm{g} / \mathrm{L} \text { ) }\end{array}$ & $\begin{array}{c}\text { Lead, } \\
\text { unfiltered } \\
\text { recoverable } \\
(\mu \mathrm{g} / \mathrm{L})\end{array}$ \\
\hline \multirow[t]{2}{*}{12323600} & \multirow[t]{2}{*}{ Silver Bow Creek at Opportunity } & $05 / 25 / 2011$ & 1030 & 2.29 & 68.9 & 310 & 182 & 7,220 & 1.47 & 101 \\
\hline & & $05 / 25 / 2011$ & 1040 & 2.23 & 65.9 & 346 & 201 & 7,410 & 1.59 & 106 \\
\hline \multirow[t]{2}{*}{12323700} & \multirow[t]{2}{*}{ Mill Creek at Opportunity } & $08 / 15 / 2011$ & 1520 & .06 & 1.6 & 2.3 & 38 & 106 & .11 & .32 \\
\hline & & $08 / 15 / 2011$ & 1525 & .06 & 1.6 & 2.3 & 40 & 105 & .11 & .32 \\
\hline \multirow[t]{2}{*}{12323750} & \multirow[t]{2}{*}{ Silver Bow Creek at Warm Springs } & $07 / 13 / 2011$ & 1010 & .09 & 3.7 & 7.4 & 45 & 272 & .18 & 1.27 \\
\hline & & $07 / 13 / 2011$ & 1015 & .09 & 3.8 & 7.5 & 48 & 279 & .19 & 1.27 \\
\hline \multirow[t]{2}{*}{12323800} & \multirow[t]{2}{*}{ Clark Fork near Galen } & $06 / 08 / 2011$ & 1145 & .38 & 20.7 & 82.1 & 47 & 1,120 & .30 & 9.10 \\
\hline & & 06/08/2011 & 1155 & .37 & 20.1 & 79.0 & 44 & 1,100 & .30 & 9.13 \\
\hline \multirow[t]{2}{*}{12323840} & \multirow[t]{2}{*}{ Lost Creek near Anaconda } & $04 / 11 / 2011$ & 1510 & $<.05$ & 1.4 & 4.6 & 7 & 115 & .02 & .48 \\
\hline & & $04 / 11 / 2011$ & 1515 & $<.05$ & 1.5 & 4.8 & 6 & 119 & .02 & .52 \\
\hline \multirow[t]{4}{*}{12324200} & \multirow[t]{4}{*}{ Clark Fork at Deer Lodge } & $05 / 17 / 2011$ & 1225 & .62 & 10.2 & 136 & 27 & 2,620 & .23 & 20.0 \\
\hline & & $05 / 17 / 2011$ & 1230 & .56 & 10.4 & 143 & 31 & 2,640 & .24 & 20.7 \\
\hline & & $06 / 24 / 2011$ & 1130 & .36 & 35.6 & 87.6 & 36 & 976 & .40 & 8.98 \\
\hline & & $06 / 24 / 2011$ & 1135 & .38 & 35.4 & 82.1 & 43 & 920 & .44 & 8.51 \\
\hline \multirow[t]{2}{*}{12324400} & \multirow{2}{*}{$\begin{array}{l}\text { Clark Fork above Little Blackfoot, } \\
\text { near Garrison }\end{array}$} & 03/15/2011 & 1500 & .17 & 6.8 & 31.9 & 6 & 586 & .07 & 4.16 \\
\hline & & $03 / 15 / 2011$ & 1505 & .19 & 7.0 & 35.2 & 8 & 587 & .08 & 4.03 \\
\hline
\end{tabular}


Table 13. Analyses of field replicates for water samples, Clark Fork basin, Montana._Continued

[Abbreviations: $\mathrm{CaCO}_{3}$, calcium carbonate; $\mu \mathrm{g} / \mathrm{L}$, micrograms per liter; $\mathrm{mg} / \mathrm{L}$, milligrams per liter; mm, millimeter. Symbol: <, less than laboratory reporting level]

\begin{tabular}{|c|c|c|c|c|c|c|c|c|c|}
\hline $\begin{array}{l}\text { Site number } \\
\text { (fig. 1) }\end{array}$ & Site name & Date & Time & $\begin{array}{c}\text { Manganese, } \\
\text { filtered } \\
(\mu \mathrm{g} / \mathrm{L})\end{array}$ & $\begin{array}{c}\text { Manganese, } \\
\text { unfiltered } \\
\text { recoverable } \\
(\mu \mathrm{g} / \mathrm{L})\end{array}$ & $\begin{array}{c}\text { Zinc, } \\
\text { filtered } \\
\text { ( } \mu \mathrm{g} / \mathrm{L})\end{array}$ & $\begin{array}{c}\text { Zinc, } \\
\text { unfiltered } \\
\text { recoverable } \\
(\mu \mathrm{g} / \mathrm{L})\end{array}$ & $\begin{array}{c}\text { Sediment } \\
\text { suspended } \\
\text { (percent finer than } \\
0.062 \mathrm{~mm} \text { ) }\end{array}$ & $\begin{array}{l}\text { Sediment, } \\
\text { suspended } \\
(\mathrm{mg} / \mathrm{L})\end{array}$ \\
\hline \multirow[t]{2}{*}{12323600} & \multirow[t]{2}{*}{ Silver Bow Creek at Opportunity } & $05 / 25 / 2011$ & 1030 & 158 & 519 & 148 & 463 & 57 & 347 \\
\hline & & $05 / 25 / 2011$ & 1040 & 156 & 544 & 145 & 461 & 50 & 419 \\
\hline \multirow[t]{2}{*}{12323700} & \multirow[t]{2}{*}{ Mill Creek at Opportunity } & $08 / 15 / 2011$ & 1520 & 7.5 & 11.0 & $<1.4$ & $<2.4$ & 80 & 2 \\
\hline & & $08 / 15 / 2011$ & 1525 & 7.6 & 10.9 & $<1.4$ & $<2.4$ & 81 & 2 \\
\hline \multirow[t]{2}{*}{12323750} & \multirow[t]{2}{*}{ Silver Bow Creek at Warm Springs } & $07 / 13 / 2011$ & 1010 & 34.3 & 62.8 & 2.1 & 7.1 & 91 & 7 \\
\hline & & $07 / 13 / 2011$ & 1015 & 36.3 & 64.2 & 2.1 & 7.1 & 93 & 7 \\
\hline \multirow[t]{2}{*}{12323800} & \multirow[t]{2}{*}{ Clark Fork near Galen } & 06/08/2011 & 1145 & 31.9 & 121 & 13.7 & 60.9 & 48 & 59 \\
\hline & & $06 / 08 / 2011$ & 1155 & 31.9 & 118 & 12.8 & 60.7 & 54 & 51 \\
\hline \multirow[t]{2}{*}{12323840} & \multirow[t]{2}{*}{ Lost Creek near Anaconda } & $04 / 11 / 2011$ & 1510 & .80 & 3.9 & $<1.4$ & $<2.4$ & 80 & 6 \\
\hline & & $04 / 11 / 2011$ & 1515 & .80 & 3.9 & $<1.4$ & $<2.4$ & 77 & 6 \\
\hline \multirow[t]{4}{*}{12324200} & \multirow[t]{4}{*}{ Clark Fork at Deer Lodge } & $05 / 17 / 2011$ & 1225 & 22.8 & 263 & 5.7 & 107 & 49 & 162 \\
\hline & & $05 / 17 / 2011$ & 1230 & 23.6 & 250 & 5.8 & 105 & 50 & 159 \\
\hline & & $06 / 24 / 2011$ & 1130 & 54.9 & 102 & 36.3 & 71.1 & 36 & 70 \\
\hline & & $06 / 24 / 2011$ & 1135 & 55.5 & 117 & 34.5 & 69.4 & 29 & 88 \\
\hline \multirow[t]{2}{*}{12324400} & \multirow{2}{*}{$\begin{array}{l}\text { Clark Fork above Little Blackfoot, } \\
\text { near Garrison }\end{array}$} & $03 / 15 / 2011$ & 1500 & 35.3 & 81.5 & 3.7 & 28.8 & 74 & 30 \\
\hline & & $03 / 15 / 2011$ & 1505 & 36.2 & 84.4 & 4.5 & 29.1 & 78 & 27 \\
\hline
\end{tabular}


Table 13. Analyses of field replicates for water samples, Clark Fork basin, Montana._Continued

[Abbreviations: $\mathrm{CaCO}_{3}$, calcium carbonate; $\mu \mathrm{g} / \mathrm{L}$, micrograms per liter; $\mathrm{mg} / \mathrm{L}$, milligrams per liter; mm, millimeter. Symbol: <, less than laboratory reporting level]

\begin{tabular}{|c|c|c|c|c|c|c|c|c|c|}
\hline $\begin{array}{l}\text { Site number } \\
\text { (fig. 1) }\end{array}$ & Site name & Date & Time & $\begin{array}{c}\text { Hardness, } \\
\text { filtered } \\
\left.\text { (mg/L as } \mathrm{CaCO}_{3}\right)\end{array}$ & $\begin{array}{c}\text { Calcium, } \\
\text { filtered } \\
\text { (mg/L) }\end{array}$ & $\begin{array}{c}\text { Magnesium } \\
\text { filtered } \\
\text { (mg/L) }\end{array}$ & $\begin{array}{c}\text { Arsenic, } \\
\text { filtered } \\
(\mu \mathrm{g} / \mathrm{L})\end{array}$ & $\begin{array}{c}\text { Arsenic, } \\
\text { unfiltered } \\
\text { recoverable } \\
(\mu \mathrm{g} / \mathrm{L})\end{array}$ & $\begin{array}{c}\text { Cadmium, } \\
\text { filtered } \\
(\mu \mathrm{g} / \mathrm{L})\end{array}$ \\
\hline \multirow[t]{2}{*}{12324680} & Clark Fork at Goldcreek & $11 / 16 / 2010$ & 1345 & 185 & 55.1 & 11.4 & 7.2 & 8.9 & 0.03 \\
\hline & & $11 / 16 / 2010$ & 1350 & 186 & 55.2 & 11.7 & 7.2 & 9.2 & .04 \\
\hline \multirow[t]{4}{*}{12334550} & Clark Fork at Turah Bridge, near & $06 / 02 / 2011$ & 1035 & 81.0 & 23.0 & 5.71 & 7.1 & 12.8 & .06 \\
\hline & Bonner & 12334550 & 1040 & 1040 & 81.5 & 23.2 & 5.69 & 7.5 & 12.7 \\
\hline & & $06 / 30 / 2011$ & 1100 & 86.1 & 24.8 & 5.89 & 12.3 & 14.3 & .08 \\
\hline & & $06 / 30 / 2011$ & 1110 & 84.3 & 24.2 & 5.81 & 12.6 & 14.0 & .08 \\
\hline \multirow[t]{2}{*}{12340000} & Blackfoot River near Bonner & $05 / 26 / 2011$ & 1200 & 81.7 & 21.1 & 7.02 & 1.0 & 2.1 & $<.02$ \\
\hline & & $05 / 26 / 2011$ & 1210 & 81.6 & 21.0 & 7.07 & 1.0 & 2.2 & $<.02$ \\
\hline \multirow[t]{4}{*}{12340500} & Clark Fork above Missoula & $06 / 09 / 2011$ & 1400 & 75.4 & 20.6 & 5.85 & 6.3 & 23.1 & .06 \\
\hline & & $06 / 09 / 2011$ & 1410 & 75.4 & 20.6 & 5.85 & 6.3 & 23.6 & .06 \\
\hline & & $06 / 15 / 2011$ & 1350 & 85.8 & 23.5 & 6.56 & 7.1 & 13.2 & .05 \\
\hline & & $06 / 15 / 2011$ & 1355 & 87.6 & 24.1 & 6.68 & 7.2 & 13.3 & .04 \\
\hline
\end{tabular}


Table 13. Analyses of field replicates for water samples, Clark Fork basin, Montana.-Continued

[Abbreviations: $\mathrm{CaCO}_{3}$, calcium carbonate; $\mu \mathrm{g} / \mathrm{L}$, micrograms per liter; $\mathrm{mg} / \mathrm{L}$, milligrams per liter; $\mathrm{mm}$, millimeter. Symbol: <, less than laboratory reporting level]

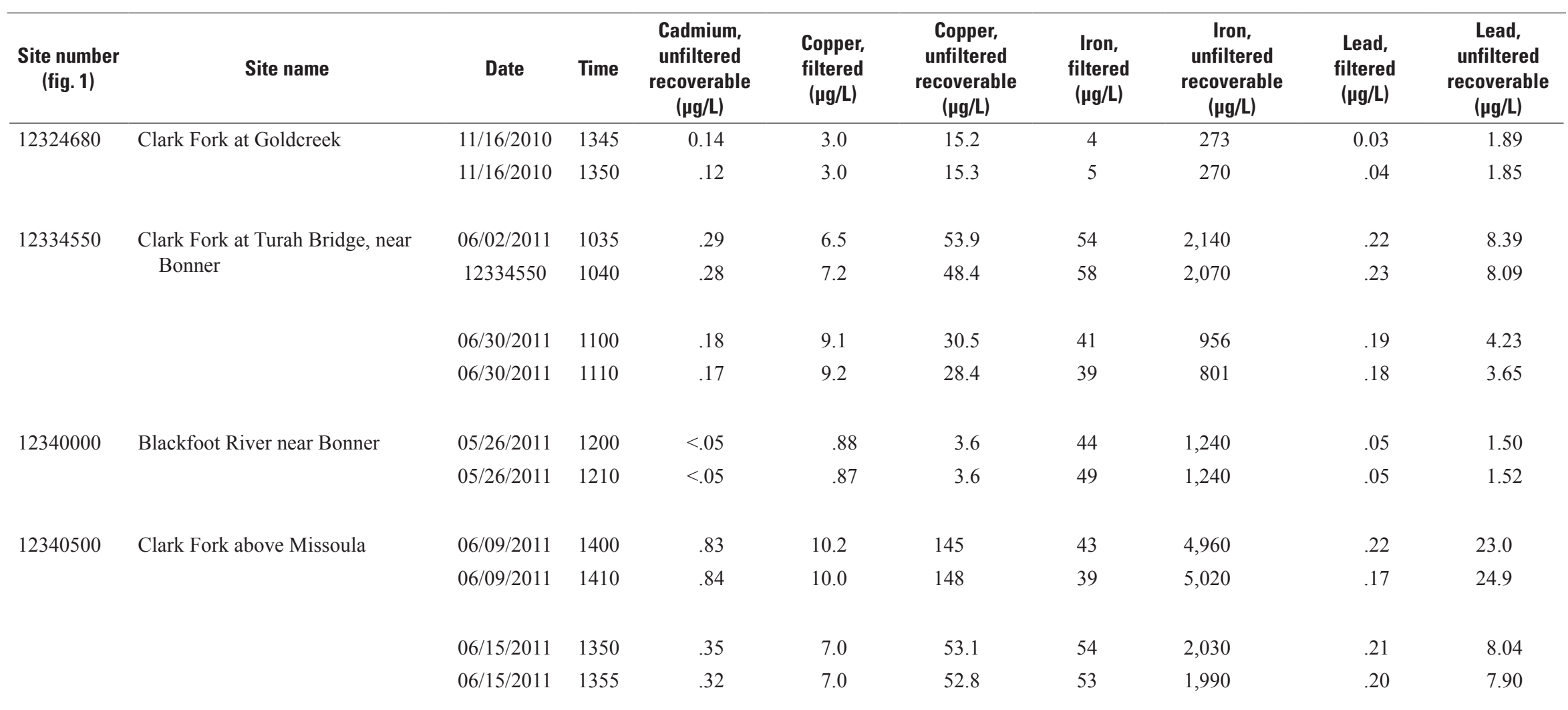


Table 13. Analyses of field replicates for water samples, Clark Fork basin, Montana._Continued

[Abbreviations: $\mathrm{CaCO}_{3}$, calcium carbonate; $\mu \mathrm{g} / \mathrm{L}$, micrograms per liter; $\mathrm{mg} / \mathrm{L}$, milligrams per liter; mm, millimeter. Symbol: <, less than laboratory reporting level]

\begin{tabular}{|c|c|c|c|c|c|c|c|c|c|}
\hline $\begin{array}{l}\text { Site number } \\
\text { (fig. 1) }\end{array}$ & Site name & Date & Time & $\begin{array}{c}\text { Manganese, } \\
\text { filtered } \\
\text { ( } \mu \mathrm{g} / \mathrm{L})\end{array}$ & $\begin{array}{c}\text { Manganese, } \\
\text { unfiltered } \\
\text { recoverable } \\
(\mu \mathrm{g} / \mathrm{L})\end{array}$ & $\begin{array}{c}\text { Zinc, } \\
\text { filtered } \\
\text { ( } \mu \mathrm{g} / \mathrm{L})\end{array}$ & $\begin{array}{c}\text { Zinc, } \\
\text { unfiltered } \\
\text { recoverable } \\
(\mu \mathrm{g} / \mathrm{L})\end{array}$ & $\begin{array}{c}\text { Sediment } \\
\text { suspended } \\
\text { (percent finer than } \\
0.062 \mathrm{~mm} \text { ) }\end{array}$ & $\begin{array}{l}\text { Sediment, } \\
\text { suspended } \\
\text { (mg/L) }\end{array}$ \\
\hline \multirow[t]{2}{*}{12324680} & Clark Fork at Goldcreek & $11 / 16 / 2010$ & 1345 & 12.6 & 58.9 & 3.5 & 17.0 & 85 & 11 \\
\hline & & $11 / 16 / 2010$ & 1350 & 12.6 & 59.8 & 3.5 & 16.4 & 81 & 12 \\
\hline \multirow[t]{4}{*}{12334550} & Clark Fork at Turah Bridge, near & $06 / 02 / 2011$ & 1035 & 13.6 & 148 & 6.4 & 70.0 & 44 & 186 \\
\hline & Bonner & 12334550 & 1040 & 14.2 & 133 & 6.6 & 64.7 & 43 & 188 \\
\hline & & 06/30/2011 & 1100 & 31.7 & 102 & 7.7 & 41.1 & 62 & 71 \\
\hline & & $06 / 30 / 2011$ & 1110 & 31.7 & 93.9 & 8.0 & 34.7 & 64 & 68 \\
\hline \multirow[t]{2}{*}{12340000} & Blackfoot River near Bonner & $05 / 26 / 2011$ & 1200 & 4.0 & 71.1 & $<1.4$ & 5.2 & 73 & 124 \\
\hline & & $05 / 26 / 2011$ & 1210 & 4.6 & 71.9 & $<1.4$ & 5.4 & 71 & 128 \\
\hline \multirow[t]{4}{*}{12340500} & Clark Fork above Missoula & $06 / 09 / 2011$ & 1400 & 26.9 & 349 & 11.9 & 210 & 60 & 427 \\
\hline & & $06 / 09 / 2011$ & 1410 & 26.5 & 347 & 9.3 & 215 & 60 & 433 \\
\hline & & $06 / 15 / 2011$ & 1350 & 20.0 & 155 & 5.5 & 84.4 & 62 & 176 \\
\hline & & $06 / 15 / 2011$ & 1355 & 20.3 & 151 & 5.8 & 84.0 & 64 & 172 \\
\hline
\end{tabular}


Table 14. Precision of analyses of field replicates for water samples, Clark Fork basin, Montana.

[Abbreviations: $\mu \mathrm{g} / \mathrm{L}$, micrograms per liter; $\mathrm{mg} / \mathrm{L}$, milligrams per liter; $\mathrm{mm}$, millimeter]

\begin{tabular}{lcccc}
\hline \multicolumn{1}{c}{ Constituent and reporting unit } & $\begin{array}{c}\text { Number of } \\
\text { replicate pairs }\end{array}$ & $\begin{array}{c}\text { Standard } \\
\text { deviation } \\
\text { (listed units) }\end{array}$ & $\begin{array}{c}\text { Relative standard } \\
\text { deviation } \\
\text { (percent) }\end{array}$ & $\begin{array}{c}\text { Within limits } \\
\text { of data-quality }^{2} \\
\text { objective }\end{array}$ \\
\hline Calcium, filtered, $\mathrm{mg} / \mathrm{L}$ & 14 & 0.62 & 2.0 & Yes \\
Magnesium, filtered, $\mathrm{mg} / \mathrm{L}$ & 14 & .22 & 3.1 & Yes \\
Arsenic, filtered, $\mu \mathrm{g} / \mathrm{L}$ & 14 & .26 & 1.9 & Yes \\
Arsenic, unfiltered recoverable, $\mu \mathrm{g} / \mathrm{L}$ & 14 & .28 & 1.4 & Yes \\
Cadmium, filtered, $\mu \mathrm{g} / \mathrm{L}$ & 14 & .00 & 4.0 & Yes \\
Cadmium, unfiltered recoverable, $\mu \mathrm{g} / \mathrm{L}$ & 14 & .02 & 4.6 & Yes \\
Copper, filtered, $\mu \mathrm{g} / \mathrm{L}$ & 14 & .60 & 4.5 & Yes \\
Copper, unfiltered recoverable, $\mu \mathrm{g} / \mathrm{L}$ & 14 & 7.2 & 10 & Yes \\
Iron, filtered, $\mu \mathrm{g} / \mathrm{L}$ & 14 & 4.3 & 9.3 & Yes \\
Iron, unfiltered recoverable, $\mu \mathrm{g} / \mathrm{L}$ & 14 & 51 & 2.9 & Yes \\
Lead, filtered, $\mu \mathrm{g} / \mathrm{L}$ & 14 & .03 & 9.7 & Yes \\
Lead, unfiltered recoverable, $\mu \mathrm{g} / \mathrm{L}$ & 14 & 1.0 & 7.4 & Yes \\
Manganese, filtered, $\mu \mathrm{g} / \mathrm{L}$ & 14 & .62 & 1.9 & Yes \\
Manganese, unfiltered recoverable, $\mu \mathrm{g} / \mathrm{L}$ & 14 & 6.9 & 4.7 & Yes \\
Zinc, filtered, $\mu \mathrm{g} / \mathrm{L}$ & 14 & .86 & 4.9 & Yes \\
Zinc, unfiltered recoverable, $\mu \mathrm{g} / \mathrm{L}$ & 14 & 1.9 & 2.3 & Yes \\
Sediment, suspended, percent finer than $0.062 \mathrm{~mm}$ & 14 & 2.6 & 4.1 & Yes \\
Sediment, suspended, $\mathrm{mg} / \mathrm{L}$ & 14 & 14 & 12 & \\
\hline
\end{tabular}

${ }^{1}$ Standard deviation is calculated using one-half the laboratory reporting level for censored values (less than the laboratory reporting level).

${ }^{2}$ Data-quality objective for an acceptable level of precision is a maximum relative standard deviation of 20 percent for field replicate analyses (table 3).

Table 15. Precision of analyses of laboratory replicates for water samples, Clark Fork basin, Montana.

[Abbreviations: $\mu \mathrm{g} / \mathrm{L}$, micrograms per liter; $\mathrm{mg} / \mathrm{L}$, milligrams per liter]

\begin{tabular}{lcccc}
\hline \multicolumn{1}{c}{ Constituent and reporting unit } & $\begin{array}{c}\text { Number of } \\
\text { replicate pairs }\end{array}$ & $\begin{array}{c}\text { Standard deviation', } \\
\text { in listed units }\end{array}$ & $\begin{array}{c}\text { Relative standard } \\
\text { deviation, } \\
\text { in percent }\end{array}$ & $\begin{array}{c}\text { Within limits } \\
\text { of data-quality } \\
\text { objective }\end{array}$ \\
\hline Calcium, filtered, $\mathrm{mg} / \mathrm{L}$ & 8 & 0.48 & 1.3 & Yes \\
Magnesium, filtered, $\mathrm{mg} / \mathrm{L}$ & 8 & .09 & 1.2 & Yes \\
Arsenic, filtered, $\mu \mathrm{g} / \mathrm{L}$ & 8 & .06 & .50 & Yes \\
Arsenic, unfiltered recoverable, $\mu \mathrm{g} / \mathrm{L}$ & 8 & .32 & 1.9 & Yes \\
Cadmium, filtered, $\mu \mathrm{g} / \mathrm{L}$ & 8 & .00 & 9.0 & Yes \\
Cadmium, unfiltered recoverable, $\mu \mathrm{g} / \mathrm{L}$ & 8 & .00 & 2.4 & Yes \\
Copper, filtered, $\mu \mathrm{g} / \mathrm{L}$ & 8 & .02 & .48 & Yes \\
Copper, unfiltered recoverable, $\mu \mathrm{g} / \mathrm{L}$ & 8 & 1.6 & 4.2 & Yes \\
Iron, filtered, $\mu \mathrm{g} / \mathrm{L}$ & 8 & .90 & 2.9 & Yes \\
Iron, unfiltered recoverable, $\mu \mathrm{g} / \mathrm{L}$ & 8 & 16 & 1.6 & Yes \\
Lead, filtered, $\mu \mathrm{g} / \mathrm{L}$ & 8 & .00 & 3.1 & Yes \\
Lead, unfiltered recoverable, $\mu \mathrm{g} / \mathrm{L}$ & 8 & .08 & 1.4 & Yes \\
Manganese, filtered, $\mu \mathrm{g} / \mathrm{L}$ & 8 & .09 & .48 & Yes \\
Manganese, unfiltered recoverable, $\mu \mathrm{g} / \mathrm{L}$ & 8 & 1.0 & 1.1 & Yes \\
Zinc, filtered, $\mu \mathrm{g} / \mathrm{L}$ & 8 & .46 & 12 & Yes \\
Zinc, unfiltered recoverable, $\mu \mathrm{g} / \mathrm{L}$ & 8 & 1.2 & 3.2 & \\
\hline
\end{tabular}

${ }^{1}$ Standard deviation is calculated using laboratory reporting level for censored values.

${ }^{2}$ Data-quality objective for an acceptable level of precision is a maximum relative standard deviation of 20 percent for laboratory replicate analyses (table 3 ). 
Table 16. Recovery efficiency for analyses of laboratory-spiked deionized-water blank samples.

[Abbreviation: $\mu \mathrm{g} / \mathrm{L}$, micrograms per liter]

\begin{tabular}{lcccc}
\hline \multicolumn{1}{c}{ Constituent and reporting unit } & $\begin{array}{c}\text { Number of } \\
\text { samples }\end{array}$ & $\begin{array}{c}\text { 95-percent confidence } \\
\text { interval for spike recovery, } \\
\text { in percent }\end{array}$ & $\begin{array}{c}\text { Mean spike recovery, } \\
\text { in percent }\end{array}$ & $\begin{array}{c}\text { Within limits' } \\
\text { of data-quality } \\
\text { objective }\end{array}$ \\
\hline Arsenic, filtered, $\mu \mathrm{g} / \mathrm{L}$ & 5 & $102-109$ & 105 & Yes \\
Arsenic, unfiltered recoverable, $\mu \mathrm{g} / \mathrm{L}$ & 5 & $90.2-99.3$ & 94.7 & Yes \\
Cadmium, filtered, $\mu \mathrm{g} / \mathrm{L}$ & 5 & $97.9-111$ & 105 & Yes \\
Cadmium, unfiltered recoverable, $\mu \mathrm{g} / \mathrm{L}$ & 5 & $92.4-99.0$ & 95.7 & Yes \\
Copper, filtered, $\mu \mathrm{g} / \mathrm{L}$ & 5 & $89.4-103$ & 96.2 & Yes \\
Copper, unfiltered recoverable, $\mu \mathrm{g} / \mathrm{L}$ & 5 & $91.6-96.2$ & 93.9 & Yes \\
Iron, filtered, $\mu \mathrm{g} / \mathrm{L}$ & 5 & $89.3-132$ & 111 & No \\
Iron, unfiltered recoverable, $\mu \mathrm{g} / \mathrm{L}$ & 5 & $98.2-117$ & 107 & Yes \\
Lead, filtered, $\mu \mathrm{g} / \mathrm{L}$ & 5 & $98.8-113$ & 106 & Yes \\
Lead, unfiltered recoverable, $\mu \mathrm{g} / \mathrm{L}$ & 5 & $98.4-101$ & 99.8 & Yes \\
Manganese, filtered, $\mu \mathrm{g} / \mathrm{L}$ & 5 & $97.0-104$ & 101 & Yes \\
Manganese, unfiltered recoverable, $\mu \mathrm{g} / \mathrm{L}$ & 5 & $97.8-100$ & 98.9 & Yes \\
Zinc, filtered, $\mu \mathrm{g} / \mathrm{L}$ & 5 & $94.3-122$ & 108 & Yes \\
Zinc, unfiltered recoverable, $\mu \mathrm{g} / \mathrm{L}$ & 5 & $92.2-100$ & 96.1 & Yes \\
\hline
\end{tabular}

${ }^{1}$ Data-quality objective for acceptable bias is a maximum deviation of 25 percent from a theoretical 100-percent recovery (table 3 ).

${ }^{2}$ Exceedance of data-quality objective resulted from one sample having a recovery of 138 percent. When data from this one spiked-sample set are removed from the 95-percent confidence interval calculation, filtered iron meets the data-quality objective limit (88.9-119 percent).

Table 17. Recovery efficiency for analyses of laboratory-spiked stream samples, Clark Fork basin, Montana.

[Abbreviation: $\mu \mathrm{g} / \mathrm{L}$, micrograms per liter]

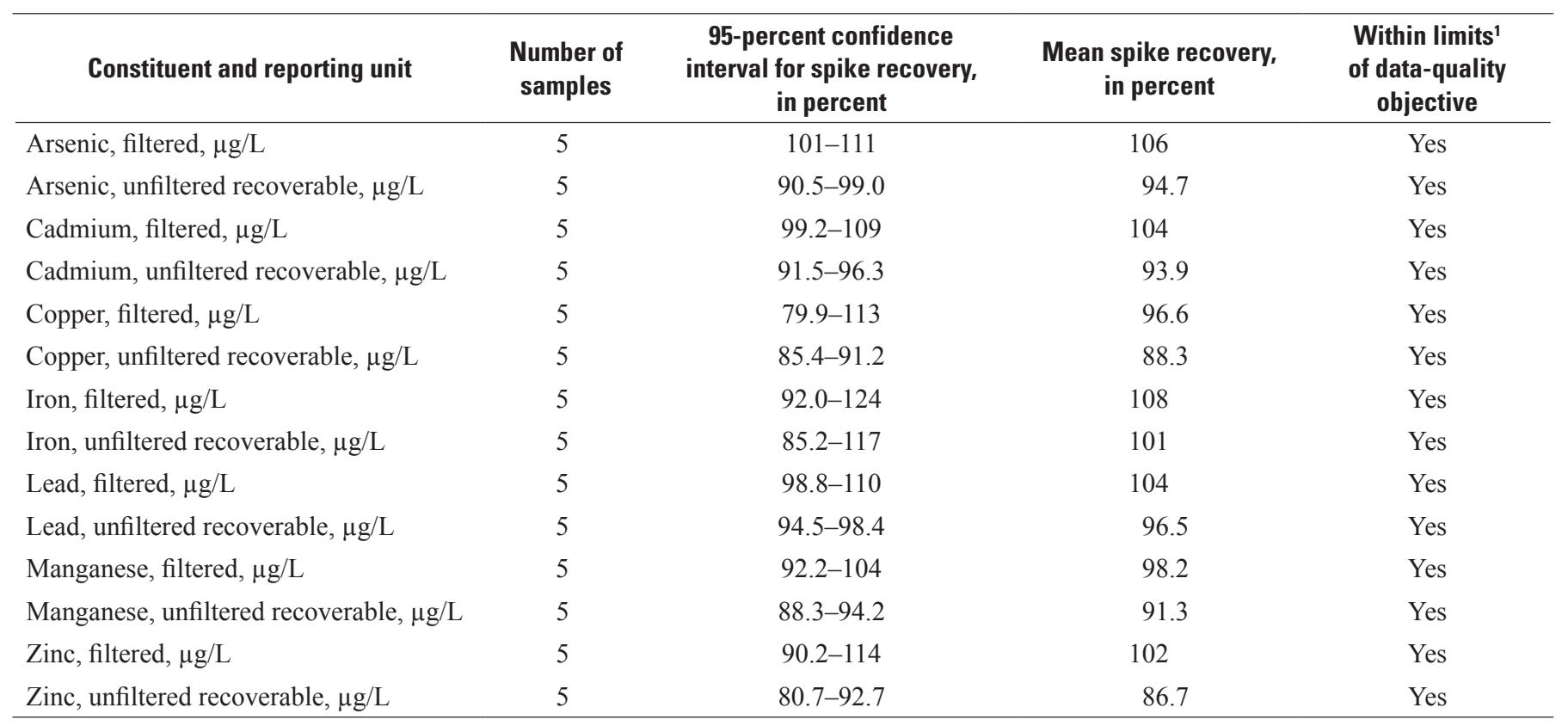

${ }^{1}$ Data-quality objective for acceptable bias is a maximum deviation of 25 percent from a theoretical 100-percent recovery (table 3 ). 
Table 18. Analyses of field blanks for water samples.

[Abbreviations: $\mu \mathrm{g} / \mathrm{L}$, micrograms per liter; $\mu \mathrm{S} / \mathrm{cm}$, microsiemens per centimeter at 25 degrees Celsius; $\mathrm{mg} / \mathrm{L}$, milligrams per liter. Symbol: $<$, less than laboratory reporting level]

\begin{tabular}{|c|c|c|c|c|c|c|c|c|c|c|}
\hline Date & Time & $\begin{array}{c}\text { pH, } \\
\text { onsite } \\
\text { (standard } \\
\text { units) } \\
\end{array}$ & $\begin{array}{c}\text { Specific } \\
\text { conductance, } \\
\text { onsite } \\
(\mu \mathrm{S} / \mathrm{cm}) \\
\end{array}$ & $\begin{array}{l}\text { Calcium, } \\
\text { filtered } \\
(\mathrm{mg} / \mathrm{L})\end{array}$ & $\begin{array}{l}\text { Magnesium, } \\
\text { filtered } \\
\text { (mg/L) }\end{array}$ & $\begin{array}{c}\text { Arsenic, } \\
\text { filtered } \\
(\mu \mathrm{g} / \mathrm{L})\end{array}$ & $\begin{array}{c}\text { Arsenic, } \\
\text { unfiltered } \\
\text { recoverable } \\
(\mu \mathrm{g} / \mathrm{L})\end{array}$ & $\begin{array}{c}\text { Cadmium, } \\
\text { filtered } \\
(\mu \mathrm{g} / \mathrm{L})\end{array}$ & $\begin{array}{c}\text { Cadmium, } \\
\text { unfiltered } \\
\text { recoverable } \\
(\mu \mathrm{g} / \mathrm{L})\end{array}$ & \\
\hline $11 / 15 / 2010$ & 1155 & 4.9 & 2 & $<0.02$ & $<0.008$ & $<0.02$ & $<0.09$ & $<0.02$ & $<0.05$ & \\
\hline *03/07/2011 & 0800 & 5.6 & 2 & .03 & $<.008$ & $<.02$ & $<.09$ & .06 & $<.05$ & \\
\hline 04/14/2011 & 1255 & 5.3 & 2 & $<.02$ & $<.008$ & $<.02$ & $<.09$ & $<.02$ & $<.05$ & \\
\hline *05/12/2011 & 0900 & 5.3 & 2 & $<.02$ & $<.008$ & $<.02$ & $<.09$ & $<.02$ & $<.05$ & \\
\hline 06/01/2011 & 1245 & 5.4 & 1 & $<.02$ & $<.008$ & $<.02$ & $<.09$ & $<.02$ & $<.05$ & \\
\hline $06 / 15 / 2011$ & 0755 & 5.5 & 1 & $<.02$ & $<.008$ & $<.02$ & $<.09$ & $<.02$ & $<.05$ & \\
\hline 06/29/2011 & 1420 & 7.1 & 2 & $<.02$ & $<.008$ & $<.02$ & $<.09$ & $<.02$ & $<.05$ & \\
\hline 07/13/2011 & 1245 & 5.6 & $<1$ & .02 & $<.008$ & $<.02$ & $<.09$ & $<.02$ & $<.05$ & \\
\hline 08/16/2011 & 0755 & 5.5 & 2 & $<.02$ & $<.008$ & $<.02$ & $<.09$ & $<.02$ & $<.05$ & \\
\hline $11 / 15 / 2010$ & $<0.50$ & $<0.70$ & $<3$ & $<5$ & $<0.01$ & $<0.04$ & $<0.1$ & $<0.4$ & $<1.4$ & $<2.4$ \\
\hline *03/07/2011 & .61 & $<.70$ & $<3$ & $<5$ & $<.01$ & $<.04$ & $<.1$ & $<.4$ & 1.9 & $<2.4$ \\
\hline 04/14/2011 & $<.50$ & $<.70$ & $<3$ & $<5$ & $<.01$ & $<.04$ & $<.1$ & $<.4$ & $<1.4$ & $<2.4$ \\
\hline *05/12/2011 & $<.50$ & $<.70$ & $<3$ & $<5$ & .02 & .05 & $<.1$ & $<.4$ & $<1.5$ & $<2.4$ \\
\hline 05/13/2011 & $<.50$ & $<.70$ & $<3$ & $<5$ & .02 & $<.04$ & .3 & $<.4$ & 1.6 & $<2.4$ \\
\hline 05/16/2011 & $<.50$ & $<.70$ & $<3$ & $<5$ & $<.01$ & $<.04$ & $<.1$ & $<.4$ & $<1.4$ & $<2.4$ \\
\hline 06/01/2011 & $<.50$ & $<.70$ & $<3$ & $<5$ & $<.01$ & $<.04$ & $<.1$ & $<.4$ & $<1.4$ & $<2.4$ \\
\hline 06/15/2011 & $<.50$ & $<.70$ & $<3$ & $<5$ & $<.01$ & $<.04$ & $<.1$ & $<.4$ & $<1.4$ & $<2.4$ \\
\hline 06/29/2011 & $<.50$ & $<.70$ & $<3$ & $<5$ & $<.01$ & $<.04$ & .3 & $<.4$ & $<1.4$ & $<2.4$ \\
\hline 07/13/2011 & $<.50$ & $<.70$ & $<3$ & $<5$ & $<.01$ & $<.04$ & $<.1$ & $<.4$ & $<1.4$ & $<2.4$ \\
\hline 08/16/2011 & $<.50$ & $<.70$ & $<3$ & $<5$ & $<.01$ & $<.04$ & $<.1$ & $<.4$ & $<1.4$ & $<2.4$ \\
\hline
\end{tabular}


Table 19. Bed-sediment data for the Clark Fork basin, Montana, August 2011.

[Trace-element concentrations in bed sediment were determined for the fine-grained fraction (material less than 0.063 millimeter in diameter). Reported concentrations are the mean of all analyses for replicate aliquots from each composite sample. Abbreviation: $\mu \mathrm{g} / \mathrm{g}$, micrograms per gram of dry sample weight]

\begin{tabular}{|c|c|c|c|c|c|c|c|c|c|c|c|}
\hline \multirow{2}{*}{$\begin{array}{l}\text { Site number } \\
\text { (fig. 1) }\end{array}$} & \multirow{2}{*}{ Site name } & \multirow{2}{*}{$\begin{array}{c}\text { Number of } \\
\text { composite } \\
\text { samples }\end{array}$} & \multicolumn{9}{|c|}{ Concentration $(\mu \mathrm{g} / \mathrm{g})$} \\
\hline & & & Arsenic & Cadmium & Chromium & Copper & Iron & Lead & Manganese & Nickel & Zinc \\
\hline 12323600 & Silver Bow Creek at Opportunity & 3 & 73 & 7.9 & 42.6 & 1,080 & 34,300 & 279 & 1,720 & 12.7 & 2,000 \\
\hline 12323750 & Silver Bow Creek at Warm Springs & 3 & 68 & 4.9 & 33.9 & 296 & 23,800 & 61 & 2,440 & 14.7 & 625 \\
\hline 12323770 & Warm Springs Creek near Warm Springs & 3 & 34 & 1.2 & 33.2 & 496 & 19,600 & 42 & 555 & 14.5 & 237 \\
\hline 12323800 & Clark Fork near Galen & 3 & 95 & 3.8 & 38.8 & 1,110 & 26,700 & 110 & 1,530 & 15.9 & 741 \\
\hline 461415112450801 & Clark Fork below Lost Creek, near Galen & 3 & 126 & 4.8 & 38.5 & 1,500 & 28,700 & 153 & 1,430 & 14.8 & 1,030 \\
\hline 461559112443301 & $\begin{array}{l}\text { Clark Fork at county bridge, near } \\
\text { Racetrack }\end{array}$ & 3 & 132 & 5.4 & 39.2 & 1,490 & 29,000 & 149 & 1,600 & 16.0 & 1,050 \\
\hline 461903112440701 & $\begin{array}{l}\text { Clark Fork at Dempsey Creek diversion, } \\
\text { near Racetrack }\end{array}$ & 3 & 90 & 4.1 & 38.0 & 1,190 & 30,100 & 141 & 1,200 & 12.7 & 961 \\
\hline 12324200 & Clark Fork at Deer Lodge & 3 & 95 & 3.9 & 45.8 & 1,200 & 31,500 & 142 & 1,260 & 15.8 & 990 \\
\hline 12324400 & $\begin{array}{l}\text { Clark Fork above Little Blackfoot River, } \\
\text { near Garrison }\end{array}$ & 3 & 91 & 4.2 & 46.4 & 1,260 & 29,100 & 144 & 1,150 & 14.9 & 1,100 \\
\hline 12324680 & Clark Fork at Goldcreek & 3 & 62 & 3.0 & 46.5 & 843 & 27,300 & 118 & 977 & 14.1 & 880 \\
\hline 12331800 & Clark Fork near Drummond & 3 & 41 & 1.7 & 36.0 & 405 & 36,800 & 75 & 832 & 12.2 & 637 \\
\hline 12334550 & Clark Fork at Turah Bridge, near Bonner & 3 & 35 & 2.0 & 37.4 & 405 & 24,100 & 69 & 1,060 & 13.6 & 724 \\
\hline 12340000 & Blackfoot River near Bonner & 3 & 4 & .05 & 23.7 & 21 & 18,300 & 12 & 564 & 10.2 & 57 \\
\hline 12340500 & Clark Fork above Missoula & 3 & 30 & 1.2 & 29.5 & 281 & 20,400 & 43 & 763 & 11.4 & 452 \\
\hline
\end{tabular}


Table 20. Recovery efficiency for analyses of standard reference materials for bed-sediment samples.

[Dilution ratio is the proportion of initial volume of concentrated nitric acid used as a digesting reagent to final volume of solution after addition of $0.6 \mathrm{~N}$ (normal) hydrochloric acid used for reconstituting dried residue. Abbreviations: $\mu \mathrm{g} / \mathrm{g}$, micrograms per gram of dry sample weight; SRM, standard reference material (agricultural soils)]

\begin{tabular}{|c|c|c|c|c|c|}
\hline Constituent & $\begin{array}{l}\text { Number of } \\
\text { analyses }\end{array}$ & $\begin{array}{c}\text { Dilution } \\
\text { ratio }\end{array}$ & $\begin{array}{c}\text { Certified } \\
\text { concentration } \\
(\mu \mathrm{g} / \mathrm{g})\end{array}$ & $\begin{array}{l}\text { Mean SRM } \\
\text { recovery } \\
\text { (percent) }\end{array}$ & $\begin{array}{c}\text { 95-percent } \\
\text { confidence interval } \\
\text { for SRM recovery } \\
\text { (percent) }\end{array}$ \\
\hline \multicolumn{6}{|c|}{ SRM sample 2709a } \\
\hline Arsenic & 10 & $1: 10$ & 10.5 & 54.0 & $51.4-56.5$ \\
\hline Cadmium & 10 & $1: 10$ & .371 & 70.7 & $67.4-74.1$ \\
\hline Chromium & 10 & $1: 10$ & 130 & 77.8 & $73.9-81.7$ \\
\hline Copper & 10 & $1: 10$ & 33.9 & 85.7 & $81.2-90.1$ \\
\hline Iron & 10 & $1: 10$ & 33,600 & 95.1 & $90.8-99.4$ \\
\hline Lead & 10 & $1: 10$ & 17.3 & 54.4 & $52.1-56.8$ \\
\hline Manganese & 10 & $1: 10$ & 529 & 93.4 & $89.2-97.6$ \\
\hline Nickel & 10 & $1: 10$ & 85 & 86.6 & $83.6-89.7$ \\
\hline Zinc & 10 & $1: 10$ & 103 & 98.1 & $94.9-101$ \\
\hline \multicolumn{6}{|c|}{ SRM sample 2711a } \\
\hline Arsenic & 10 & $1: 10$ & 107 & 84.6 & $82.1-87.1$ \\
\hline Cadmium & 10 & $1: 10$ & 54.1 & 96.5 & $93.5-99.6$ \\
\hline Chromium & 10 & $1: 10$ & 52 & 75.3 & $70.9-79.8$ \\
\hline Copper & 10 & $1: 10$ & 1,410 & 104 & $99.0-109$ \\
\hline Iron & 10 & $1: 10$ & 28,200 & 88.5 & $84.4-92.7$ \\
\hline Lead & 10 & $1: 10$ & 1,400 & 88.5 & $86.1-90.8$ \\
\hline Manganese & 10 & $1: 10$ & 675 & 82.6 & $78.9-86.3$ \\
\hline Nickel & 10 & $1: 10$ & 21.7 & 86.0 & $83.5-88.5$ \\
\hline Zinc & 10 & $1: 10$ & 414 & 98.8 & $95.4-102$ \\
\hline
\end{tabular}


Table 21. Analyses of procedural blanks for bed-sediment samples.

[Dilution ratio is the proportion of initial volume of concentrated nitric acid used as a digesting reagent to final volume of solution after addition of $0.6 \mathrm{~N}$ (normal) hydrochloric acid used for reconstituting dried residue. Abbreviation: $\mu \mathrm{g} / \mathrm{mL}$, micrograms per milliliter. Symbol: $<$, less than minimum reporting level for liquid-phase concentration, in $\mu \mathrm{g} / \mathrm{mL}]$

\begin{tabular}{|c|c|c|c|c|c|c|c|c|c|c|c|}
\hline \multirow{2}{*}{$\begin{array}{l}\text { Site number } \\
\text { (fig. 1) }\end{array}$} & \multirow{2}{*}{ Site name } & \multirow{2}{*}{$\begin{array}{l}\text { Dilution } \\
\text { ratio }\end{array}$} & \multicolumn{9}{|c|}{ Trace-element concentration $(\mu \mathrm{g} / \mathrm{mL})$} \\
\hline & & & Arsenic & Cadmium & Chromium & Copper & Iron & Lead & Manganese & Nickel & Zinc \\
\hline 12323600 & $\begin{array}{l}\text { Silver Bow Creek at } \\
\text { Opportunity }\end{array}$ & $1: 10$ & $<0.004$ & $<0.0002$ & $<0.003$ & $<0.003$ & $<0.03$ & $<0.003$ & $<0.001$ & $<0.002$ & $<0.02$ \\
\hline 12323750 & $\begin{array}{l}\text { Silver Bow Creek at Warm } \\
\text { Springs }\end{array}$ & $1: 10$ & $<.004$ & $<.0002$ & $<.003$ & $<.003$ & $<.03$ & $<.003$ & $<.001$ & $<.002$ & $<.02$ \\
\hline 12323770 & $\begin{array}{l}\text { Warm Springs Creek at } \\
\text { Warm Springs }\end{array}$ & $1: 10$ & $<.004$ & $<.0002$ & $<.003$ & $<.003$ & $<.03$ & $<.003$ & $<.001$ & $<.002$ & $<.02$ \\
\hline 12323800 & Clark Fork near Galen & $1: 10$ & $<.004$ & $<.0002$ & $<.003$ & $<.003$ & $<.03$ & $<.003$ & $<.001$ & $<.002$ & $<.02$ \\
\hline 461415112450801 & $\begin{array}{l}\text { Clark Fork below Lost } \\
\text { Creek, near Galen }\end{array}$ & $1: 10$ & $<.004$ & $<.0002$ & $<.003$ & $<.003$ & $<.03$ & $<.003$ & $<.001$ & $<.002$ & $<.02$ \\
\hline 461559112443301 & $\begin{array}{l}\text { Clark Fork at county bridge, } \\
\text { near Racetrack }\end{array}$ & $1: 10$ & $<.004$ & $<.0002$ & $<.003$ & $<.003$ & $<.03$ & $<.003$ & $<.001$ & $<.002$ & $<.02$ \\
\hline 461903112440701 & $\begin{array}{l}\text { Clark Fork at Dempsey } \\
\text { Creek diversion, near } \\
\text { Racetrack }\end{array}$ & $1: 10$ & $<.004$ & $<.0002$ & $<.003$ & $<.003$ & $<.03$ & $<.003$ & $<.001$ & $<.002$ & $<.02$ \\
\hline 12324200 & Clark Fork at Deer Lodge & $1: 10$ & $<.004$ & $<.0002$ & $<.003$ & $<.003$ & $<.03$ & $<.003$ & $<.001$ & $<.002$ & $<.02$ \\
\hline 12324400 & $\begin{array}{l}\text { Clark Fork above Little } \\
\text { Blackfoot River, near } \\
\text { Garrison }\end{array}$ & $1: 10$ & $<.004$ & $<.0002$ & $<.003$ & $<.003$ & $<.03$ & $<.003$ & $<.001$ & $<.002$ & $<.02$ \\
\hline 12324680 & Clark Fork at Goldcreek & $1: 10$ & $<.004$ & $<.0002$ & $<.003$ & $<.003$ & $<.03$ & $<.003$ & $<.001$ & $<.002$ & $<.02$ \\
\hline 12331800 & Clark Fork near Drummond & $1: 10$ & $<.004$ & $<.0002$ & $<.003$ & $<.003$ & $<.03$ & $<.003$ & $<.001$ & $<.002$ & $<.02$ \\
\hline 12334550 & $\begin{array}{l}\text { Clark Fork at Turah Bridge, } \\
\text { near Bonner }\end{array}$ & $1: 10$ & $<.004$ & $<.0002$ & $<.003$ & $<.003$ & $<.03$ & $<.003$ & $<.001$ & $<.002$ & $<.02$ \\
\hline 12340000 & Blackfoot River near Bonner & $1: 10$ & $<.004$ & $<.0002$ & $<.003$ & $<.003$ & $<.03$ & $<.003$ & $<.001$ & $<.002$ & $<.02$ \\
\hline 12340500 & Clark Fork above Missoula & $1: 10$ & $<.004$ & $<.0002$ & $<.003$ & $<.003$ & $<.03$ & $<.003$ & $<.001$ & $<.002$ & $<.02$ \\
\hline
\end{tabular}


Table 22. Biological data for the Clark Fork basin, Montana, August 2011.

[Analyses are for the whole-body tissue of aquatic insects. Composite samples were made by combining similar-sized insects of the same species into a sample of sufficient mass for analysis. Concentrations for biota samples composed of two or more composite samples are the means of all analyses. Abbreviations: $\mu \mathrm{g} / \mathrm{g}$, micrograms per gram of dry sample weight; spp., species. Symbol: $<$, less than minimum reporting level for solid-phase concentration, in $\mu \mathrm{g} / \mathrm{g}]$

\begin{tabular}{|c|c|c|c|c|c|c|c|c|c|c|}
\hline \multirow[b]{2}{*}{ Taxon } & \multirow{2}{*}{$\begin{array}{c}\text { Number of } \\
\text { composite } \\
\text { samples }\end{array}$} & \multicolumn{9}{|c|}{ Concentration $(\mu \mathrm{g} / \mathrm{g})$} \\
\hline & & Arsenic & Cadmium & Chromium & Copper & Iron & Lead & $\begin{array}{c}\text { Manga- } \\
\text { nese }\end{array}$ & Nickel & Zinc \\
\hline Hydropsyche cockerelli & 1 & 21.4 & 9.3 & 25.5 & 522 & 6,150 & 74.3 & 1,500 & 3.6 & 1,470 \\
\hline Hydropsyche spp. & 2 & 9.0 & 2.7 & 3.8 & 214 & 2,910 & 27.7 & 697 & 1.4 & 571 \\
\hline Hydropsyche cockerelli & 1 & 16.7 & 0.9 & 2.2 & 45.3 & 1,650 & 6.0 & 1,010 & 1.4 & 169 \\
\hline Hydropsyche occidentalis & 1 & 14.3 & .9 & 2.2 & 46.9 & 1,610 & 5.6 & 996 & 1.3 & 170 \\
\hline \multicolumn{11}{|c|}{ 12323770--Warm Springs Creek at Warm Springs } \\
\hline \multicolumn{11}{|c|}{ 12323800--Clark Fork near Galen } \\
\hline Hydropsyche cockerelli & 1 & 16.9 & 2.0 & 9.6 & 119 & 1,580 & 17.1 & 3,360 & 3.3 & 363 \\
\hline Hydropsyche occidentalis & 3 & 11.2 & 1.2 & 3.0 & 131 & 2,310 & 9.4 & 705 & 2.2 & 212 \\
\hline \multicolumn{11}{|c|}{ 461415112450801--Clark Fork below Lost Creek, near Galen } \\
\hline Rhyacophila spp. & 2 & 4.3 & 4.1 & 1.1 & 83.4 & 335 & 5.4 & 256 & 0.3 & 356 \\
\hline \multicolumn{11}{|c|}{ 461559112443301--Clark Fork at county bridge, near Racetrack } \\
\hline Hydropsyche occidentalis & 3 & 18.7 & 1.3 & 3.4 & 199 & 2,930 & 18.2 & 777 & 2.0 & 271 \\
\hline \multicolumn{11}{|c|}{ 12324400--Clark Fork above Little Blackfoot River, near Garrison } \\
\hline Arctopsyche grandis & 1 & 16.6 & 3.2 & 4.6 & 209 & 2,580 & 18.0 & 972 & 2.2 & 378 \\
\hline Hydropsyche occidentalis & 2 & 13.0 & 1.5 & 3.5 & 165 & 2,370 & 15.5 & 1,080 & 1.7 & 275 \\
\hline Hydropsyche spp. & 1 & 13.6 & 1.7 & 4.3 & 187 & 2,570 & 18.5 & 919 & 1.8 & 296 \\
\hline \multicolumn{11}{|c|}{ 12324680--Clark Fork at Goldcreek } \\
\hline Arctopsyche grandis & 3 & 14.1 & 2.6 & 4.9 & 201 & 2,850 & 15.2 & 1,020 & 2.7 & 299 \\
\hline Claassenia sabulosa & 2 & 2.0 & 1.4 & 1.3 & 74.5 & 532 & 2.3 & 132 & .3 & 275 \\
\hline Hydropsyche occidentalis & 2 & 11.4 & 1.1 & 3.6 & 159 & 2,380 & 13.1 & 734 & 1.7 & 216 \\
\hline
\end{tabular}


Table 22. Biological data for the Clark Fork basin, Montana, August 2011.—Continued

[Analyses are for the whole-body tissue of aquatic insects. Composite samples were made by combining similar-sized insects of the same species into a sample of sufficient mass for analysis. Concentrations for biota samples composed of two or more composite samples are the means of all analyses. Abbreviations: $\mu \mathrm{g} / \mathrm{g}$, micrograms per gram of dry sample weight; spp., species. Symbol: <, less than minimum reporting level for solid-phase concentration, in $\mu \mathrm{g} / \mathrm{g}$ ]

\begin{tabular}{|c|c|c|c|c|c|c|c|c|c|c|}
\hline \multirow[b]{2}{*}{ Taxon } & \multirow{2}{*}{$\begin{array}{c}\text { Number of } \\
\text { composite } \\
\text { samples }\end{array}$} & \multicolumn{9}{|c|}{ Concentration $(\mu \mathrm{g} / \mathrm{g})$} \\
\hline & & Arsenic & Cadmium & Chromium & Copper & Iron & Lead & $\begin{array}{c}\text { Manga- } \\
\text { nese }\end{array}$ & Nickel & Zinc \\
\hline Arctopsyche grandis & 2 & 8.0 & 3.4 & 3.8 & 102 & 1,710 & 11.6 & 1,010 & 1.5 & 307 \\
\hline Claassenia sabulosa & 2 & 1.8 & 2.0 & 1.0 & 74.2 & 438 & 1.9 & 143 & .3 & 345 \\
\hline \multicolumn{11}{|c|}{ 12334550--Clark Fork at Turah Bridge, near Bonner } \\
\hline Arctopsyche grandis & 2 & 6.6 & 2.6 & 3.8 & 80.2 & 1,990 & 9.6 & 813 & 1.6 & 271 \\
\hline Claassenia sabulosa & 2 & .9 & 1.6 & 1.1 & 63.8 & 279 & 1.2 & 178 & .2 & 250 \\
\hline Hydropsyche cockerelli & 1 & 9.8 & 2.2 & 14.2 & 126 & 3,180 & 19.7 & 848 & 2.7 & 332 \\
\hline Arctopsyche grandis & 1 & 1.9 & 0.5 & 5.6 & 18.0 & 1,880 & 1.9 & 624 & 2.4 & 152 \\
\hline Claassenia sabulosa & 2 & $<.12$ & .2 & .8 & 49.5 & 237 & .1 & 77 & .3 & 232 \\
\hline Hydropsyche occidentalis & 3 & 1.5 & .3 & 2.3 & 17.9 & 1,800 & 1.4 & 435 & 1.5 & 124 \\
\hline \multicolumn{11}{|c|}{ 12340500--Clark Fork above Missoula } \\
\hline Arctopsyche grandis & 1 & 4.9 & 1.6 & 3.4 & 60.5 & 2,050 & 5.9 & 751 & 1.6 & 215 \\
\hline Claassenia sabulosa & 2 & 1.1 & .6 & .8 & 51.8 & 247 & .7 & 129 & .2 & 225 \\
\hline Hydropsyche occidentalis & 2 & 5.2 & .9 & 3.3 & 66.2 & 2,200 & 6.3 & 769 & 1.6 & 195 \\
\hline
\end{tabular}


Table 23. Recovery efficiency for analyses of standard reference material for biota samples.

[Abbreviations: $\mu \mathrm{g} / \mathrm{g}$, micrograms per gram of dry sample weight; SRM, standard reference material (lobster hepatopancreas)]

\begin{tabular}{lcccc}
\hline Constituent & $\begin{array}{c}\text { Number of } \\
\text { analyses }\end{array}$ & $\begin{array}{c}\text { Certified } \\
\text { concentration } \\
(\boldsymbol{\mu g} / \mathbf{g})\end{array}$ & $\begin{array}{c}\text { Mean } \\
\text { SRM recovery } \\
\text { (percent) }\end{array}$ & $\begin{array}{c}\text { 95-percent confidence } \\
\text { interval for SRM recovery } \\
\text { (percent) }\end{array}$ \\
\hline Arsenic & 12 & \multicolumn{2}{c}{ SRM sample TORT-2 } & \\
Cadmium & 12 & 21.6 & 95.5 & $94.5-96.6$ \\
Chromium & 12 & 26.7 & 77.4 & $74.2-80.6$ \\
Copper & 12 & .77 & 129 & $123-136$ \\
Iron & 12 & 106 & 92.7 & $90.6-94.8$ \\
Lead & 12 & .35 & 90.0 & $86.9-92.6$ \\
Manganese & 12 & 13.6 & 78.1 & $72.0-84.2$ \\
Nickel & 12 & 2.5 & 89.8 & $86.9-92.8$ \\
Zinc & 12 & 180 & 71.6 & $69.1-74.1$ \\
\hline
\end{tabular}


Table 24. Analyses of procedural blanks for biota samples.

[Procedural blanks were not diluted prior to analyses. Abbreviation: $\mu \mathrm{g} / \mathrm{mL}$, micrograms per milliliter. Symbol: <, less than minimum reporting level for liquid-phase concentration, in $\mu \mathrm{g} / \mathrm{mL}$ ]

\begin{tabular}{|c|c|c|c|c|c|c|c|c|c|c|c|}
\hline \multirow{2}{*}{$\begin{array}{l}\text { Site number } \\
\text { (fig. 1) }\end{array}$} & \multirow{2}{*}{ Site name } & \multirow{2}{*}{$\begin{array}{l}\text { Dilution } \\
\text { ratio }\end{array}$} & \multicolumn{9}{|c|}{ Trace-element concentration $(\mu \mathrm{g} / \mathrm{mL})$} \\
\hline & & & Arsenic & Cadmium & Chromium & Copper & Iron & Lead & Manganese & Nickel & Zinc \\
\hline 12323600 & Silver Bow Creek at Opportunity & $1: 1$ & $<0.0013$ & $<0.0002$ & $<0.0009$ & $<0.0014$ & $<0.01$ & $<0.002$ & $<0.0012$ & $<0.001$ & $<0.003$ \\
\hline 12323750 & $\begin{array}{l}\text { Silver Bow Creek at Warm } \\
\text { Springs }\end{array}$ & $1: 1$ & $<.0013$ & $<.0002$ & $<.0009$ & $<.0014$ & $<.01$ & $<.002$ & $<.0012$ & $<.001$ & $<.003$ \\
\hline 12323770 & $\begin{array}{l}\text { Warm Springs Creek at Warm } \\
\text { Springs }\end{array}$ & $1: 1$ & $<.0013$ & $<.0002$ & $<.0009$ & $<.0014$ & $<.01$ & $<.002$ & $<.0012$ & $<.001$ & $<.003$ \\
\hline 12323800 & Clark Fork near Galen & $1: 1$ & $<.0013$ & $<.0002$ & $<.0009$ & $<.0014$ & $<.01$ & $<.002$ & $<.0012$ & $<.001$ & $<.003$ \\
\hline 461415112450801 & $\begin{array}{l}\text { Clark Fork below Lost Creek, } \\
\text { near Galen }\end{array}$ & $1: 1$ & $<.0013$ & $<.0002$ & $<.0009$ & $<.0014$ & $<.01$ & $<.002$ & $<.0012$ & $<.001$ & $<.003$ \\
\hline 461559112443301 & $\begin{array}{l}\text { Clark Fork at county bridge, near } \\
\text { Racetrack }\end{array}$ & $1: 1$ & $<.0013$ & $<.0002$ & $<.0009$ & $<.0014$ & $<.01$ & $<.002$ & $<.0012$ & $<.001$ & $<.003$ \\
\hline 461903112440701 & $\begin{array}{l}\text { Clark Fork at Dempsey Creek } \\
\text { diversion, near Racetrack }\end{array}$ & $1: 1$ & $<.0013$ & $<.0002$ & $<.0009$ & $<.0014$ & $<.01$ & $<.002$ & $<.0012$ & $<.001$ & $<.003$ \\
\hline 12324200 & Clark Fork at Deer Lodge & $1: 1$ & $<.0013$ & $<.0002$ & $<.0009$ & $<.0014$ & $<.01$ & $<.002$ & $<.0012$ & $<.001$ & $<.003$ \\
\hline 12324400 & $\begin{array}{l}\text { Clark Fork above Little } \\
\text { Blackfoot River, near Garrison }\end{array}$ & $1: 1$ & $<.0013$ & $<.0002$ & $<.0009$ & $<.0014$ & $<.01$ & $<.002$ & $<.0012$ & $<.001$ & $<.003$ \\
\hline 12324680 & Clark Fork at Goldcreek & $1: 1$ & $<.0013$ & $<.0002$ & $<.0009$ & $<.0014$ & $<.01$ & $<.002$ & $<.0012$ & $<.001$ & $<.003$ \\
\hline 12331800 & Clark Fork near Drummond & $1: 1$ & $<.0013$ & $<.0002$ & $<.0009$ & $<.0014$ & $<.01$ & $<.002$ & $<.0012$ & $<.001$ & $<.003$ \\
\hline 12334550 & $\begin{array}{l}\text { Clark Fork at Turah Bridge, near } \\
\text { Bonner }\end{array}$ & $1: 1$ & $<.0013$ & $<.0002$ & $<.0009$ & $<.0014$ & $<.01$ & $<.002$ & $<.0012$ & $<.001$ & $<.003$ \\
\hline 12340000 & Blackfoot River near Bonner & $1: 1$ & $<.0013$ & $<.0002$ & $<.0009$ & $<.0014$ & $<.01$ & $<.002$ & $<.0012$ & $<.001$ & $<.003$ \\
\hline 12340500 & Clark Fork above Missoula & $1: 1$ & $<.0013$ & $<.0002$ & $<.0009$ & $<.0014$ & $<.01$ & $<.002$ & $<.0012$ & $<.04$ & $<.003$ \\
\hline
\end{tabular}


Table 25. Statistical summary of long-term water-quality data for the Clark Fork basin, Montana, March 1985 through September 2011.

[Abbreviations: $\mathrm{CaCO}_{3}$, calcium carbonate; $\mathrm{ft} / \mathrm{s}$, cubic feet per second; ${ }^{\circ} \mathrm{C}$, degrees Celsius; $\mu \mathrm{g} / \mathrm{L}$, micrograms per liter; $\mu \mathrm{S} / \mathrm{cm}, \mathrm{microsiemens}$ per centimeter at $25^{\circ} \mathrm{C} ; \mathrm{mg} / \mathrm{L}$, milligrams per liter; $\mathrm{mm}$, millimeter; ton/d, tons per day. Symbols: <, less than laboratory reporting level $\left.{ }^{1}\right]$

\begin{tabular}{|c|c|c|c|c|c|}
\hline Property or constituent and reporting unit & $\begin{array}{l}\text { Number of } \\
\text { samples }\end{array}$ & Maximum & Minimum & Mean & Median \\
\hline \multicolumn{6}{|c|}{$\begin{array}{l}\text { 12323230--Blacktail Creek at Harrison Avenue, at Butte } \\
\text { lata: March 1993-August 1995, December 1996-August } 200\end{array}$} \\
\hline Streamflow, instantaneous $\left(\mathrm{ft}^{3} / \mathrm{s}\right)$ & 139 & 156 & 1.9 & 16 & 9.2 \\
\hline pH, onsite (standard units) & 139 & 8.4 & 7.3 & 7.8 & 7.7 \\
\hline Specific conductance, onsite $(\mu \mathrm{S} / \mathrm{cm})$ & 139 & 412 & 116 & 261 & 259 \\
\hline Temperature, water $\left({ }^{\circ} \mathrm{C}\right)$ & 139 & 17.5 & 1.0 & 8.1 & 8.5 \\
\hline Hardness, filtered (mg/L as $\left.\mathrm{CaCO}_{3}\right)$ & 139 & 146 & 37.7 & 103 & 104 \\
\hline Calcium, filtered (mg/L) & 139 & 41.8 & 10.6 & 29.4 & 29.8 \\
\hline Magnesium, filtered (mg/L) & 139 & 11.0 & 2.71 & 7.14 & 7.16 \\
\hline Arsenic, filtered $(\mu \mathrm{g} / \mathrm{L})$ & 138 & 13.0 & 1.0 & 4.2 & 3.6 \\
\hline Arsenic, unfiltered recoverable $(\mu \mathrm{g} / \mathrm{L})$ & 139 & 18.0 & 1.0 & ${ }^{2} 5.6$ & 4.9 \\
\hline Cadmium, filtered $(\mu \mathrm{g} / \mathrm{L})$ & 137 & .50 & $<.04$ & ${ }^{2} .05$ & .02 \\
\hline Cadmium, unfiltered recoverable $(\mu \mathrm{g} / \mathrm{L})$ & 139 & .11 & $<.01$ & ${ }^{2} .04$ & $<1$ \\
\hline Copper, filtered $(\mu \mathrm{g} / \mathrm{L})$ & 138 & 10.0 & $<1.0$ & 23.7 & 3.2 \\
\hline Copper, unfiltered recoverable $(\mu \mathrm{g} / \mathrm{L})$ & 139 & 52.0 & 1.5 & 6.7 & 5.2 \\
\hline Iron, filtered $(\mu \mathrm{g} / \mathrm{L})$ & 139 & 640 & 15 & 188 & 171 \\
\hline Iron, unfiltered recoverable $(\mu \mathrm{g} / \mathrm{L})$ & 139 & 4,220 & 139 & 674 & 590 \\
\hline Lead, filtered $(\mu \mathrm{g} / \mathrm{L})$ & 139 & 2.80 & $<.08$ & ${ }^{2} .20$ & $<1.00$ \\
\hline Lead, unfiltered recoverable $(\mu \mathrm{g} / \mathrm{L})$ & 139 & 47.0 & $<1.00$ & ${ }^{2} 1.78$ & .72 \\
\hline Manganese, filtered $(\mu \mathrm{g} / \mathrm{L})$ & 139 & 144 & 14.2 & 42.1 & 37.5 \\
\hline Manganese, unfiltered recoverable $(\mu \mathrm{g} / \mathrm{L})$ & 139 & 240 & 23.5 & 59.5 & 51.3 \\
\hline Zinc, filtered $(\mu \mathrm{g} / \mathrm{L})$ & 137 & 11 & $<1.0$ & 23.5 & 2.8 \\
\hline Zinc, unfiltered recoverable $(\mu \mathrm{g} / \mathrm{L})$ & 139 & 130 & $<10$ & 28.5 & 4.0 \\
\hline Sediment, suspended (percent finer than $0.062 \mathrm{~mm}$ ) & 139 & 97 & 50 & 82 & 83 \\
\hline Sediment, suspended concentration (mg/L) & 139 & 139 & 1 & 12 & 7 \\
\hline Sediment, suspended discharge (ton/d) & 139 & 59 & .01 & 1.1 & .18 \\
\hline
\end{tabular}


Table 25. Statistical summary of long-term water-quality data for the Clark Fork basin, Montana, March 1985 through September 2011.-Continued

[Abbreviations: $\mathrm{CaCO}_{3}$, calcium carbonate; $\mathrm{ft} / \mathrm{s}$, cubic feet per second; ${ }^{\circ} \mathrm{C}$, degrees Celsius; $\mu \mathrm{g} / \mathrm{L}$, micrograms per liter; $\mu \mathrm{S} / \mathrm{cm}$, microsiemens per centimeter at $25^{\circ} \mathrm{C} ; \mathrm{mg} / \mathrm{L}$, milligrams per liter; $\mathrm{mm}$, millimeter; ton/d, tons per day. Symbols: $<$, less than laboratory reporting level $\left.{ }^{1}\right]$

\begin{tabular}{|c|c|c|c|c|c|}
\hline Property or constituent and reporting unit & $\begin{array}{l}\text { Number of } \\
\text { samples }\end{array}$ & Maximum & Minimum & Mean & Median \\
\hline \multicolumn{6}{|c|}{$\begin{array}{l}\text { 12323250--Silver Bow Creek below Blacktail Creek, at Butte } \\
\text { Period of record for water-quality data: March 1993-August 1995, December 1996-August 2003, December 2004-September } 2011\end{array}$} \\
\hline Streamflow, instantaneous $\left(\mathrm{ft}^{3} / \mathrm{s}\right)$ & 147 & 202 & 13 & 31 & 25 \\
\hline pH, onsite (standard units) & 147 & 8.2 & 7.2 & 7.6 & 7.6 \\
\hline Specific conductance, onsite $(\mu \mathrm{S} / \mathrm{cm})$ & 147 & 691 & 209 & 458 & 468 \\
\hline Temperature, water $\left({ }^{\circ} \mathrm{C}\right)$ & 147 & 20.0 & 1.0 & 10.3 & 9.5 \\
\hline Hardness, filtered ( $\mathrm{mg} / \mathrm{L}$ as $\mathrm{CaCO}_{3}$ ) & 147 & 217 & 66.0 & 146 & 150 \\
\hline Calcium, filtered $(\mathrm{mg} / \mathrm{L})$ & 147 & 62.7 & 19.0 & 41.7 & 42.4 \\
\hline Magnesium, filtered (mg/L) & 147 & 14.6 & 4.51 & 10.3 & 10.5 \\
\hline Arsenic, filtered $(\mu \mathrm{g} / \mathrm{L})$ & 147 & 13.4 & 2.3 & 6.3 & 6.0 \\
\hline Arsenic, unfiltered recoverable $(\mu \mathrm{g} / \mathrm{L})$ & 147 & 45.0 & 3.0 & 10.3 & 9.0 \\
\hline Cadmium, filtered ( $\mu \mathrm{g} / \mathrm{L})$ & 147 & 6.2 & .04 & .95 & .50 \\
\hline Cadmium, unfiltered recoverable $(\mu \mathrm{g} / \mathrm{L})$ & 147 & 6.0 & .09 & 1.29 & .90 \\
\hline Copper, filtered $(\mu \mathrm{g} / \mathrm{L})$ & 147 & 303 & 3.2 & 32.3 & 13.2 \\
\hline Copper, unfiltered recoverable $(\mu \mathrm{g} / \mathrm{L})$ & 147 & 550 & 8.9 & 74.5 & 27.1 \\
\hline Iron, filtered $(\mu \mathrm{g} / \mathrm{L})$ & 147 & 292 & 10 & 94.5 & 76.0 \\
\hline Iron, unfiltered recoverable $(\mu \mathrm{g} / \mathrm{L})$ & 147 & 7,400 & 85 & 842 & 570 \\
\hline Lead, filtered $(\mu \mathrm{g} / \mathrm{L})$ & 147 & 2.4 & $<.5$ & 2.44 & .24 \\
\hline Lead, unfiltered recoverable $(\mu \mathrm{g} / \mathrm{L})$ & 147 & 250 & .64 & 11.5 & 3.00 \\
\hline Manganese, filtered $(\mu \mathrm{g} / \mathrm{L})$ & 147 & 1,700 & 21.4 & 317 & 236 \\
\hline Manganese, unfiltered recoverable $(\mu \mathrm{g} / \mathrm{L})$ & 147 & 1,600 & 25.9 & 360 & 289 \\
\hline Zinc, filtered $(\mu \mathrm{g} / \mathrm{L})$ & 147 & 2,200 & 5.3 & 311 & 182 \\
\hline Zinc, unfiltered recoverable $(\mu \mathrm{g} / \mathrm{L})$ & 147 & 2,200 & 21.2 & 377 & 230 \\
\hline Sediment, suspended (percent finer than $0.062 \mathrm{~mm}$ ) & 146 & 98 & 42 & 84 & 85 \\
\hline Sediment, suspended concentration $(\mathrm{mg} / \mathrm{L})$ & 146 & 405 & 2 & 21 & 10 \\
\hline Sediment, suspended discharge (ton/d) & 146 & 70 & .08 & 2.5 & .68 \\
\hline
\end{tabular}


Table 25. Statistical summary of long-term water-quality data for the Clark Fork basin, Montana, March 1985 through September 2011.-Continued

[Abbreviations: $\mathrm{CaCO}_{3}$, calcium carbonate; $\mathrm{ft} / \mathrm{s}$, cubic feet per second; ${ }^{\circ} \mathrm{C}$, degrees Celsius; $\mu \mathrm{g} / \mathrm{L}$, micrograms per liter; $\mu \mathrm{S} / \mathrm{cm}$, microsiemens per centimeter at $25^{\circ} \mathrm{C} ; \mathrm{mg} / \mathrm{L}$, milligrams per liter; $\mathrm{mm}$, millimeter; ton/d, tons per day. Symbols: $<$, less than laboratory reporting level $\left.{ }^{1}\right]$

\begin{tabular}{|c|c|c|c|c|c|}
\hline Property or constituent and reporting unit & $\begin{array}{l}\text { Number of } \\
\text { samples }\end{array}$ & Maximum & Minimum & Mean & Median \\
\hline \multicolumn{6}{|c|}{$\begin{array}{l}\text { 12323600--Silver Bow Creek at Opportunity } \\
\text { Period of record for water-quality data: March 1993-August 1995, December 1996-September } 2011\end{array}$} \\
\hline Streamflow, instantaneous $\left(\mathrm{ft}^{3} / \mathrm{s}\right)$ & 149 & 648 & 13 & 81 & 52 \\
\hline pH, onsite (standard units) & 149 & 9.5 & 7.2 & 8.4 & 8.3 \\
\hline Specific conductance, onsite $(\mu \mathrm{S} / \mathrm{cm})$ & 149 & 633 & 202 & 410 & 400 \\
\hline Temperature, water $\left({ }^{\circ} \mathrm{C}\right)$ & 149 & 22.5 & 0.0 & 9.4 & 9.5 \\
\hline Hardness, filtered (mg/L as $\mathrm{CaCO}_{3}$ ) & 149 & 240 & 60.2 & 147 & 143 \\
\hline Calcium, filtered (mg/L) & 149 & 71.6 & 18.5 & 43.5 & 43.0 \\
\hline Magnesium, filtered (mg/L) & 149 & 15.0 & 3.42 & 9.31 & 9.01 \\
\hline Arsenic, filtered $(\mu \mathrm{g} / \mathrm{L})$ & 149 & 34.0 & 1.0 & 10.9 & 10.3 \\
\hline Arsenic, unfiltered recoverable $(\mu \mathrm{g} / \mathrm{L})$ & 149 & 235 & 9.1 & 24.6 & 17.0 \\
\hline Cadmium, filtered $(\mu \mathrm{g} / \mathrm{L})$ & 148 & 41.0 & $<.1$ & ${ }^{2} 1.05$ & .58 \\
\hline Cadmium, unfiltered recoverable $(\mu \mathrm{g} / \mathrm{L})$ & 149 & 49.0 & .34 & ${ }^{2} 1.90$ & 1.18 \\
\hline Copper, filtered $(\mu \mathrm{g} / \mathrm{L})$ & 147 & 450 & 12.0 & 43.0 & 32.7 \\
\hline Copper, unfiltered recoverable $(\mu \mathrm{g} / \mathrm{L})$ & 149 & 3,900 & 26.9 & 188 & 100 \\
\hline Iron, filtered $(\mu \mathrm{g} / \mathrm{L})$ & 149 & 307 & $<3$ & 249 & 27 \\
\hline Iron, unfiltered recoverable $(\mu \mathrm{g} / \mathrm{L})$ & 148 & 24,100 & 240 & 1,540 & 780 \\
\hline Lead, filtered $(\mu \mathrm{g} / \mathrm{L})$ & 149 & 5.1 & $<.5$ & ${ }^{2} .70$ & .33 \\
\hline Lead, unfiltered recoverable $(\mu \mathrm{g} / \mathrm{L})$ & 149 & 650 & 3.27 & 35.4 & 14.9 \\
\hline Manganese, filtered $(\mu \mathrm{g} / \mathrm{L})$ & 149 & 9,300 & 30.3 & 394 & 302 \\
\hline Manganese, unfiltered recoverable $(\mu \mathrm{g} / \mathrm{L})$ & 149 & 10,000 & 69.8 & 512 & 404 \\
\hline Zinc, filtered $(\mu \mathrm{g} / \mathrm{L})$ & 148 & 13,000 & 11.2 & 271 & 137 \\
\hline Zinc, unfiltered recoverable $(\mu \mathrm{g} / \mathrm{L})$ & 149 & 15,000 & 52.5 & 478 & 260 \\
\hline Sediment, suspended (percent finer than $0.062 \mathrm{~mm}$ ) & 150 & 95 & 37 & 78 & 82 \\
\hline Sediment, suspended concentration (mg/L) & 150 & 801 & 5 & 50 & 20 \\
\hline Sediment, suspended discharge (ton/d) & 149 & 781 & .18 & 23 & 2.7 \\
\hline
\end{tabular}


Table 25. Statistical summary of long-term water-quality data for the Clark Fork basin, Montana, March 1985 through September 2011.-Continued

[Abbreviations: $\mathrm{CaCO}_{3}$, calcium carbonate; $\mathrm{ft} / \mathrm{s}$, cubic feet per second; ${ }^{\circ} \mathrm{C}$, degrees Celsius; $\mu \mathrm{g} / \mathrm{L}$, micrograms per liter; $\mu \mathrm{S} / \mathrm{cm}$, microsiemens per centimeter at $25^{\circ} \mathrm{C} ; \mathrm{mg} / \mathrm{L}$, milligrams per liter; $\mathrm{mm}$, millimeter; ton/d, tons per day. Symbols: $<$, less than laboratory reporting level $\left.{ }^{1}\right]$

\begin{tabular}{|c|c|c|c|c|c|}
\hline Property or constituent and reporting unit & $\begin{array}{l}\text { Number of } \\
\text { samples }\end{array}$ & Maximum & Minimum & Mean & Median \\
\hline \multicolumn{6}{|c|}{$\begin{array}{c}\text { 12323670--Mill Creek near Anaconda } \\
\text { Period of record for water-quality data: December 2004-September } 2011\end{array}$} \\
\hline Streamflow, instantaneous $\left(\mathrm{ft}^{3} / \mathrm{s}\right)$ & 56 & 309 & 7.4 & 65 & 30 \\
\hline $\mathrm{pH}$, onsite (standard units) & 56 & 8.6 & 7.5 & 8.0 & 8.0 \\
\hline Specific conductance, onsite $(\mu \mathrm{S} / \mathrm{cm})$ & 56 & 213 & 56 & 131 & 132 \\
\hline Temperature, water $\left({ }^{\circ} \mathrm{C}\right)$ & 56 & 17.0 & 0.0 & 8.3 & 8.0 \\
\hline Hardness, filtered (mg/L as $\mathrm{CaCO}_{3}$ ) & 56 & 97.5 & 23.7 & 56.6 & 57.1 \\
\hline Calcium, filtered $(\mathrm{mg} / \mathrm{L})$ & 56 & 25.9 & 7.00 & 15.7 & 16.0 \\
\hline Magnesium, filtered (mg/L) & 56 & 8.01 & 1.45 & 4.24 & 4.18 \\
\hline Arsenic, filtered $(\mu \mathrm{g} / \mathrm{L})$ & 56 & 32.9 & 7.3 & 16.9 & 15.3 \\
\hline Arsenic, unfiltered recoverable $(\mu \mathrm{g} / \mathrm{L})$ & 56 & 34.8 & 8.6 & 18.4 & 16.9 \\
\hline Cadmium, filtered $(\mu \mathrm{g} / \mathrm{L})$ & 55 & .11 & .02 & ${ }^{2} .04$ & .04 \\
\hline Cadmium, unfiltered recoverable $(\mu \mathrm{g} / \mathrm{L})$ & 56 & .19 & .03 & .08 & .07 \\
\hline Copper, filtered $(\mu \mathrm{g} / \mathrm{L})$ & 56 & 5.1 & .72 & 2.3 & 2.1 \\
\hline Copper, unfiltered recoverable $(\mu \mathrm{g} / \mathrm{L})$ & 56 & 10.6 & 1.3 & 4.0 & 3.5 \\
\hline Iron, filtered $(\mu \mathrm{g} / \mathrm{L})$ & 56 & 125 & 21 & 46 & 40 \\
\hline Iron, unfiltered recoverable $(\mu \mathrm{g} / \mathrm{L})$ & 56 & 619 & 78 & 201 & 162 \\
\hline Lead, filtered $(\mu \mathrm{g} / \mathrm{L})$ & 56 & .24 & $<.08$ & ${ }^{2} .12$ & .11 \\
\hline Lead, unfiltered recoverable $(\mu \mathrm{g} / \mathrm{L})$ & 56 & 3.12 & .15 & .79 & .59 \\
\hline Manganese, filtered $(\mu \mathrm{g} / \mathrm{L})$ & 56 & 11.9 & 3.1 & 5.8 & 5.6 \\
\hline Manganese, unfiltered recoverable $(\mu \mathrm{g} / \mathrm{L})$ & 56 & 36.6 & 7.4 & 14.1 & 12.6 \\
\hline Zinc, filtered $(\mu \mathrm{g} / \mathrm{L})$ & 56 & 4.0 & $<1.4$ & ${ }^{2} 1.6$ & 1.3 \\
\hline Zinc, unfiltered recoverable $(\mu \mathrm{g} / \mathrm{L})$ & 56 & 9.2 & 1.0 & ${ }^{2} 3.1$ & 2.6 \\
\hline Sediment, suspended (percent finer than $0.062 \mathrm{~mm}$ ) & 56 & 83 & 28 & 63 & 66 \\
\hline Sediment, suspended concentration $(\mathrm{mg} / \mathrm{L})$ & 56 & 29 & 1 & 7 & 4 \\
\hline Sediment, suspended discharge (ton/d) & 56 & 13 & .02 & 2.1 & .28 \\
\hline
\end{tabular}


Table 25. Statistical summary of long-term water-quality data for the Clark Fork basin, Montana, March 1985 through September 2011.-Continued

[Abbreviations: $\mathrm{CaCO}_{3}$, calcium carbonate; $\mathrm{ft} / \mathrm{s}$, cubic feet per second; ${ }^{\circ} \mathrm{C}$, degrees Celsius; $\mu \mathrm{g} / \mathrm{L}$, micrograms per liter; $\mu \mathrm{S} / \mathrm{cm}$, microsiemens per centimeter at $25^{\circ} \mathrm{C} ; \mathrm{mg} / \mathrm{L}$, milligrams per liter; $\mathrm{mm}$, millimeter; ton/d, tons per day. Symbols: $<$, less than laboratory reporting level $\left.{ }^{1}\right]$

\begin{tabular}{lccccc}
\hline \multicolumn{1}{c}{ Property or constituent and reporting unit } & $\begin{array}{c}\text { Number of } \\
\text { samples }\end{array}$ & Maximum & Minimum & Mean & Median \\
\hline \multicolumn{1}{c}{ Period of record for water-quality data: March 2003-September 2011 } & & \\
\hline Streamflow, instantaneous $(\mathrm{ft} / \mathrm{s})$ & 72 & 261 & 0.43 & 39 & 12 \\
pH, onsite (standard units) & 72 & 8.2 & 7.5 & 8.0 & 8.0 \\
Specific conductance, onsite $(\mu \mathrm{S} / \mathrm{cm})$ & 72 & 230 & 59 & 147 & 152 \\
Temperature, water $\left({ }^{\circ} \mathrm{C}\right)$ & 72 & 20.0 & 0.0 & 9.2 & 9.0 \\
Hardness, filtered $\left(\mathrm{mg} / \mathrm{L}\right.$ as $\left.\mathrm{CaCO}_{3}\right)$ & 72 & 102 & 24.0 & 62.5 & 65.0 \\
Calcium, filtered $(\mathrm{mg} / \mathrm{L})$ & 72 & 28.0 & 7.01 & 17.5 & 18.2 \\
Magnesium, filtered $(\mathrm{mg} / \mathrm{L})$ & 72 & 7.83 & 1.56 & 4.54 & 4.67 \\
Arsenic, filtered $(\mu \mathrm{g} / \mathrm{L})$ & 72 & 55.1 & 9.0 & 22.4 & 20.6 \\
Arsenic, unfiltered recoverable $(\mu \mathrm{g} / \mathrm{L})$ & 72 & 53.5 & 10.0 & 25.7 & 24.5 \\
Cadmium, filtered $(\mu \mathrm{g} / \mathrm{L})$ & 72 & .13 & .02 & .06 & .06 \\
Cadmium, unfiltered recoverable $(\mu \mathrm{g} / \mathrm{L})$ & 72 & .85 & .04 & .14 & .10 \\
Copper, filtered $(\mu \mathrm{g} / \mathrm{L})$ & 72 & 6.1 & 1.0 & 3.0 & 2.6 \\
Copper, unfiltered recoverable $(\mu \mathrm{g} / \mathrm{L})$ & 72 & 38.8 & 1.5 & 6.8 & 4.4 \\
Iron, filtered $(\mu \mathrm{g} / \mathrm{L})$ & 72 & 94 & 16 & 47 & 42 \\
Iron, unfiltered recoverable $(\mu \mathrm{g} / \mathrm{L})$ & 72 & 1,960 & 44 & 314 & 145 \\
Lead, filtered $(\mu \mathrm{g} / \mathrm{L})$ & 72 & .35 & $<.08$ & 2.14 & .13 \\
Lead, unfiltered recoverable $(\mu \mathrm{g} / \mathrm{L})$ & 72 & 12.7 & .07 & 1.54 & .44 \\
Manganese, filtered $(\mu \mathrm{g} / \mathrm{L})$ & 72 & 32.8 & 2.1 & 7.3 & 5.5 \\
Manganese, unfiltered recoverable $(\mu \mathrm{g} / \mathrm{L})$ & 72 & 113 & 3.2 & 19.4 & 13.0 \\
Zinc, filtered $(\mu \mathrm{g} / \mathrm{L})$ & 71 & 7.7 & 1.3 & 3.0 & 2.8 \\
Zinc, unfiltered recoverable $(\mu \mathrm{g} / \mathrm{L})$ & 72 & 41 & 1.7 & 6.8 & 5.0 \\
Sediment, suspended $($ percent finer than 0.062 mm) & 72 & 90 & 26 & 68 & 72 \\
Sediment, suspended concentration $(\mathrm{mg} / \mathrm{L})$ & 107 & 1 & 13 & 2 \\
Sediment, suspended discharge $(\mathrm{ton} / \mathrm{d})$ & 55 & $<.01$ & 23.8 & .06
\end{tabular}


Table 25. Statistical summary of long-term water-quality data for the Clark Fork basin, Montana, March 1985 through September 2011.-Continued

[Abbreviations: $\mathrm{CaCO}_{3}$, calcium carbonate; $\mathrm{ft} / \mathrm{s}$, cubic feet per second; ${ }^{\circ} \mathrm{C}$, degrees Celsius; $\mu \mathrm{g} / \mathrm{L}$, micrograms per liter; $\mu \mathrm{S} / \mathrm{cm}$, microsiemens per centimeter at $25^{\circ} \mathrm{C} ; \mathrm{mg} / \mathrm{L}$, milligrams per liter; $\mathrm{mm}$, millimeter; ton/d, tons per day. Symbols: $<$, less than laboratory reporting level $\left.{ }^{1}\right]$

\begin{tabular}{lccccc}
\hline \multicolumn{1}{c}{ Property or constituent and reporting unit } & $\begin{array}{c}\text { Number of } \\
\text { samples }\end{array}$ & Maximum & Minimum & Mean & Median \\
\hline \multicolumn{1}{c}{ Period of record for water-quality data: } & December 2004-September 2011 & & \\
\hline Streamflow, instantaneous $\left(\mathrm{ft}^{3} / \mathrm{s}\right)$ & 51 & 114 & 1.0 & 17 & 7.7 \\
$\mathrm{pH}$, onsite $($ standard units) & 51 & 8.2 & 7.5 & 7.7 & 7.7 \\
Specific conductance, onsite $(\mu \mathrm{S} / \mathrm{cm})$ & 51 & 151 & 66 & 103 & 108 \\
Temperature, water $\left({ }^{\circ} \mathrm{C}\right)$ & 51 & 15.5 & .5 & 7.0 & 6.5 \\
Hardness, filtered $\left(\mathrm{mg} / \mathrm{L}\right.$ as $\left.\mathrm{CaCO}_{3}\right)$ & 51 & 56.3 & 22.1 & 37.4 & 38.5 \\
Calcium, filtered $(\mathrm{mg} / \mathrm{L})$ & 51 & 18.3 & 7.56 & 12.5 & 13.0 \\
Magnesium, filtered $(\mathrm{mg} / \mathrm{L})$ & 51 & 2.60 & .78 & 1.48 & 1.44 \\
Arsenic, filtered $(\mu \mathrm{g} / \mathrm{L})$ & 51 & 24.9 & 9.9 & 15.8 & 14.7 \\
Arsenic, unfiltered recoverable $(\mu \mathrm{g} / \mathrm{L})$ & 51 & 27.0 & 9.8 & 16.9 & 15.6 \\
Cadmium, filtered $(\mu \mathrm{g} / \mathrm{L})$ & 49 & .05 & $<.04$ & 2.03 & .03 \\
Cadmium, unfiltered recoverable $(\mu \mathrm{g} / \mathrm{L})$ & 51 & .33 & $<.04$ & 2.07 & .05 \\
Copper, filtered $(\mu \mathrm{g} / \mathrm{L})$ & 51 & 4.2 & .90 & 2.1 & 2.0 \\
Copper, unfiltered recoverable $(\mu \mathrm{g} / \mathrm{L})$ & 51 & 16.8 & 1.0 & 4.0 & 3.2 \\
Iron, filtered $(\mu \mathrm{g} / \mathrm{L})$ & 51 & 277 & 28 & 84 & 66 \\
Iron, unfiltered recoverable $(\mu \mathrm{g} / \mathrm{L})$ & 51 & 2,380 & 86 & 392 & 226 \\
Lead, filtered $(\mu \mathrm{g} / \mathrm{L})$ & 51 & .37 & .03 & 2.15 & .14 \\
Lead, unfiltered recoverable $(\mu \mathrm{g} / \mathrm{L})$ & 51 & 7.96 & .10 & 1.00 & .49 \\
Manganese, filtered $(\mu \mathrm{g} / \mathrm{L})$ & 51 & 34.5 & 6.0 & 14.6 & 13.2 \\
Manganese, unfiltered recoverable $(\mu \mathrm{g} / \mathrm{L})$ & 51 & 99.9 & 14.0 & 28.1 & 24.2 \\
Zinc, filtered $(\mu \mathrm{g} / \mathrm{L})$ & 51 & 3.3 & .65 & 21.7 & 1.6 \\
Zinc, unfiltered recoverable $(\mu \mathrm{g} / \mathrm{L})$ & 51 & 17.8 & $<2.0$ & 23.5 & 2.2 \\
Sediment, suspended $($ percent finer than $0.062 \mathrm{~mm})$ & 51 & 94 & 25 & 72 & 78 \\
Sediment, suspended concentration $(\mathrm{mg} / \mathrm{L})$ & 51 & 195 & 1 & 19 & 6 \\
Sediment, suspended discharge $(\mathrm{ton} / \mathrm{d})$ & 51 & 50 & $<.01$ & 22.8 & .14
\end{tabular}


Table 25. Statistical summary of long-term water-quality data for the Clark Fork basin, Montana, March 1985 through September 2011.-Continued

[Abbreviations: $\mathrm{CaCO}_{3}$, calcium carbonate; $\mathrm{ft} / \mathrm{s}$, cubic feet per second; ${ }^{\circ} \mathrm{C}$, degrees Celsius; $\mu \mathrm{g} / \mathrm{L}$, micrograms per liter; $\mu \mathrm{S} / \mathrm{cm}$, microsiemens per centimeter at $25^{\circ} \mathrm{C} ; \mathrm{mg} / \mathrm{L}$, milligrams per liter; $\mathrm{mm}$, millimeter; ton/d, tons per day. Symbols: <, less than laboratory reporting level $\left.{ }^{1}\right]$

\begin{tabular}{lccccc}
\hline \multicolumn{1}{c}{ Property or constituent and reporting unit } & $\begin{array}{c}\text { Number of } \\
\text { samples }\end{array}$ & Maximum & Minimum & Mean & Median \\
\hline \multicolumn{1}{c}{ Period of record for water-quality data: March 2003-September 2011 } & & \\
\hline Streamflow, instantaneous $(\mathrm{ft} / \mathrm{s})$ & 72 & 116 & 4.5 & 18 & 9.1 \\
$\mathrm{pH}$, onsite (standard units) & 72 & 9.0 & 7.7 & 8.1 & 8.1 \\
Specific conductance, onsite $(\mu \mathrm{S} / \mathrm{cm})$ & 72 & 371 & 116 & 271 & 291 \\
Temperature, water $\left({ }^{\circ} \mathrm{C}\right)$ & 72 & 20.5 & 1.5 & 11.2 & 11.2 \\
Hardness, filtered $\left(\mathrm{mg} / \mathrm{L}\right.$ as $\left.\mathrm{CaCO}_{3}\right)$ & 72 & 169 & 58.5 & 117 & 126 \\
Calcium, filtered $(\mathrm{mg} / \mathrm{L})$ & 72 & 47.4 & 18.1 & 34.1 & 36.4 \\
Magnesium, filtered $(\mathrm{mg} / \mathrm{L})$ & 72 & 12.3 & 3.24 & 7.78 & 8.24 \\
Arsenic, filtered $(\mu \mathrm{g} / \mathrm{L})$ & 72 & 164 & 10.9 & 41.1 & 32.0 \\
Arsenic, unfiltered recoverable $(\mu \mathrm{g} / \mathrm{L})$ & 72 & 164 & 12.0 & 44.0 & 33.2 \\
Cadmium, filtered $(\mu \mathrm{g} / \mathrm{L})$ & 72 & .12 & $<.04$ & 2.04 & .04 \\
Cadmium, unfiltered recoverable $(\mu \mathrm{g} / \mathrm{L})$ & 72 & .52 & .02 & .11 & .07 \\
Copper, filtered $(\mu \mathrm{g} / \mathrm{L})$ & 72 & 21.4 & 1.1 & 5.5 & 3.4 \\
Copper, unfiltered recoverable $(\mu \mathrm{g} / \mathrm{L})$ & 72 & 48.8 & 2.8 & 11.9 & 8.0 \\
Iron, filtered $(\mu \mathrm{g} / \mathrm{L})$ & 72 & 274 & 7.0 & 50 & 42 \\
Iron, unfiltered recoverable $(\mu \mathrm{g} / \mathrm{L})$ & 72 & 1,670 & 27 & 303 & 216 \\
Lead, filtered $(\mu \mathrm{g} / \mathrm{L})$ & 72 & .89 & .04 & 2.23 & .18 \\
Lead, unfiltered recoverable $(\mu \mathrm{g} / \mathrm{L})$ & 72 & 14.4 & .27 & 2.45 & 1.62 \\
Manganese, filtered $(\mu \mathrm{g} / \mathrm{L})$ & 72 & 200 & 3.3 & 32.1 & 24.0 \\
Manganese, unfiltered recoverable $(\mu \mathrm{g} / \mathrm{L})$ & 72 & 228 & 4.7 & 46.0 & 36.4 \\
Zinc, filtered $(\mu \mathrm{g} / \mathrm{L})$ & 72 & 19.8 & $<1.4$ & 25.2 & 4.0 \\
Zinc, unfiltered recoverable $(\mu \mathrm{g} / \mathrm{L})$ & 72 & 68 & 1.1 & 13.2 & 9.8 \\
Sediment, suspended $($ percent finer than 0.062 & $\mathrm{mm})$ & 97 & 54 & 84 & 86 \\
Sediment, suspended concentration $(\mathrm{mg} / \mathrm{L})$ & 72 & 11 & .02 & 12 & 6 \\
Sediment, suspended discharge $($ ton/d) & 72 & & & .1 & .16
\end{tabular}


Table 25. Statistical summary of long-term water-quality data for the Clark Fork basin, Montana, March 1985 through September 2011.-Continued

[Abbreviations: $\mathrm{CaCO}_{3}$, calcium carbonate; $\mathrm{ft} / \mathrm{s}$, cubic feet per second; ${ }^{\circ} \mathrm{C}$, degrees Celsius; $\mu \mathrm{g} / \mathrm{L}$, micrograms per liter; $\mu \mathrm{S} / \mathrm{cm}$, microsiemens per centimeter at $25^{\circ} \mathrm{C} ; \mathrm{mg} / \mathrm{L}$, milligrams per liter; $\mathrm{mm}$, millimeter; ton/d, tons per day. Symbols: $<$, less than laboratory reporting level $\left.{ }^{1}\right]$

\begin{tabular}{|c|c|c|c|c|c|}
\hline Property or constituent and reporting unit & $\begin{array}{l}\text { Number of } \\
\text { samples }\end{array}$ & Maximum & Minimum & Mean & Median \\
\hline \multicolumn{6}{|c|}{$\begin{array}{l}\text { 12323750--Silver Bow Creek at Warm Springs } \\
\text { Period of record for water-quality data: March 1993-September } 2011\end{array}$} \\
\hline Streamflow, instantaneous $\left(\mathrm{ft}^{3} / \mathrm{s}\right)$ & 156 & 1,030 & 16 & 145 & 88 \\
\hline pH, onsite (standard units) & 154 & 9.6 & 8.0 & 8.8 & 8.8 \\
\hline Specific conductance, onsite $(\mu \mathrm{S} / \mathrm{cm})$ & 154 & 783 & 182 & 464 & 476 \\
\hline Temperature, water $\left({ }^{\circ} \mathrm{C}\right)$ & 155 & 25.0 & .5 & 10.6 & 10.5 \\
\hline Hardness, filtered $\left(\mathrm{mg} / \mathrm{L}\right.$ as $\left.\mathrm{CaCO}_{3}\right)$ & 154 & 314 & 74.9 & 192 & 196 \\
\hline Calcium, filtered $(\mathrm{mg} / \mathrm{L})$ & 154 & 90.4 & 22.5 & 56.1 & 57.3 \\
\hline Magnesium, filtered (mg/L) & 154 & 21.4 & 4.52 & 12.7 & 13.0 \\
\hline Arsenic, filtered $(\mu \mathrm{g} / \mathrm{L})$ & 154 & 60.0 & 6.8 & 23.1 & 23.4 \\
\hline Arsenic, unfiltered recoverable $(\mu \mathrm{g} / \mathrm{L})$ & 154 & 94.0 & 10.0 & 26.9 & 26.4 \\
\hline Cadmium, filtered $(\mu \mathrm{g} / \mathrm{L})$ & 154 & .31 & $<.04$ & ${ }^{2} .06$ & .03 \\
\hline Cadmium, unfiltered recoverable $(\mu \mathrm{g} / \mathrm{L})$ & 154 & .56 & $<.1$ & ${ }^{2} .12$ & .06 \\
\hline Copper, filtered $(\mu \mathrm{g} / \mathrm{L})$ & 154 & 40.0 & 1.7 & 7.9 & 5.8 \\
\hline Copper, unfiltered recoverable $(\mu \mathrm{g} / \mathrm{L})$ & 154 & 96.8 & 2.4 & 15.8 & 11.3 \\
\hline Iron, filtered $(\mu \mathrm{g} / \mathrm{L})$ & 154 & 93 & $<5$ & ${ }^{2} 18$ & 15 \\
\hline Iron, unfiltered recoverable $(\mu \mathrm{g} / \mathrm{L})$ & 154 & 3,000 & 36 & 321 & 247 \\
\hline Lead, filtered $(\mu \mathrm{g} / \mathrm{L})$ & 154 & 1.0 & $<.08$ & ${ }^{2} .12$ & $<1.0$ \\
\hline Lead, unfiltered recoverable $(\mu \mathrm{g} / \mathrm{L})$ & 154 & 41.8 & $<1$ & 22.30 & 1.24 \\
\hline Manganese, filtered $(\mu \mathrm{g} / \mathrm{L})$ & 154 & 875 & 11.8 & 118 & 78.6 \\
\hline Manganese, unfiltered recoverable $(\mu \mathrm{g} / \mathrm{L})$ & 154 & 899 & 24.0 & 178 & 140 \\
\hline Zinc, filtered $(\mu \mathrm{g} / \mathrm{L})$ & 154 & 73 & $<1.0$ & 27.4 & 4.0 \\
\hline Zinc, unfiltered recoverable $(\mu \mathrm{g} / \mathrm{L})$ & 154 & 180 & 2.0 & 230.2 & 18.0 \\
\hline Sediment, suspended (percent finer than $0.062 \mathrm{~mm}$ ) & 155 & 98 & 43 & 82 & 85 \\
\hline Sediment, suspended concentration (mg/L) & 156 & 229 & 1 & 10 & 6 \\
\hline Sediment, suspended discharge (ton/d) & 156 & 279 & .07 & 6.4 & 1.4 \\
\hline
\end{tabular}


Table 25. Statistical summary of long-term water-quality data for the Clark Fork basin, Montana, March 1985 through September 2011.-Continued

[Abbreviations: $\mathrm{CaCO}_{3}$, calcium carbonate; $\mathrm{ft} / \mathrm{s}$, cubic feet per second; ${ }^{\circ} \mathrm{C}$, degrees Celsius; $\mu \mathrm{g} / \mathrm{L}$, micrograms per liter; $\mu \mathrm{S} / \mathrm{cm}$, microsiemens per centimeter at $25^{\circ} \mathrm{C} ; \mathrm{mg} / \mathrm{L}$, milligrams per liter; $\mathrm{mm}$, millimeter; ton/d, tons per day. Symbols: $<$, less than laboratory reporting level $\left.{ }^{1}\right]$

\begin{tabular}{lccccc}
\hline \multicolumn{1}{c}{ Property or constituent and reporting unit } & $\begin{array}{c}\text { Number of } \\
\text { samples }\end{array}$ & Maximum & Minimum & Mean & Median \\
\hline \multicolumn{1}{c}{ Period of record for water-quality data: } & October 2005-September 2011 & & \\
\hline Streamflow, instantaneous $(\mathrm{ft} / \mathrm{s})$ & 36 & 573 & 41 & 148 & 88 \\
$\mathrm{pH}$, onsite $($ standard units) & 36 & 8.8 & 7.8 & 8.5 & 8.5 \\
Specific conductance, onsite $(\mu \mathrm{S} / \mathrm{cm})$ & 36 & 271 & 125 & 217 & 230 \\
Temperature, water $\left({ }^{\circ} \mathrm{C}\right)$ & 36 & 16.0 & 4.0 & 8.9 & 8.5 \\
Hardness, filtered $\left(\mathrm{mg} / \mathrm{L}\right.$ as $\left.\mathrm{CaCO}_{3}\right)$ & 36 & 134 & 58.5 & 105 & 110 \\
Calcium, filtered $(\mathrm{mg} / \mathrm{L})$ & 36 & 39.2 & 18.5 & 31.6 & 33.2 \\
Magnesium, filtered $(\mathrm{mg} / \mathrm{L})$ & 36 & 9.07 & 2.96 & 6.39 & 6.72 \\
Arsenic, filtered $(\mu \mathrm{g} / \mathrm{L})$ & 36 & 3.9 & 1.8 & 2.3 & 2.2 \\
Arsenic, unfiltered recoverable $(\mu \mathrm{g} / \mathrm{L})$ & 36 & 5.6 & 2.0 & 2.8 & 2.6 \\
Cadmium, filtered $(\mu \mathrm{g} / \mathrm{L})$ & 36 & .05 & $<.02$ & 2.02 & .02 \\
Cadmium, unfiltered recoverable $(\mu \mathrm{g} / \mathrm{L})$ & 36 & .14 & $<.04$ & 2.04 & .03 \\
Copper, filtered $(\mu \mathrm{g} / \mathrm{L})$ & 36 & 6.4 & $<1.0$ & 21.2 & .88 \\
Copper, unfiltered recoverable $(\mu \mathrm{g} / \mathrm{L})$ & 36 & 28.0 & 1.1 & 23.9 & 2.1 \\
Iron, filtered $(\mu \mathrm{g} / \mathrm{L})$ & 36 & 22 & $<6$ & 28 & 6 \\
Iron, unfiltered recoverable $(\mu \mathrm{g} / \mathrm{L})$ & 36 & 1,000 & 27 & 141 & 76 \\
Lead, filtered $(\mu \mathrm{g} / \mathrm{L})$ & 36 & .11 & $<.01$ & 2.03 & $<.12$ \\
Lead, unfiltered recoverable $(\mu \mathrm{g} / \mathrm{L})$ & 36 & 3.51 & .07 & .50 & .26 \\
Manganese, filtered $(\mu \mathrm{g} / \mathrm{L})$ & 36 & 2.9 & $<.1$ & 21.2 & 1.0 \\
Manganese, unfiltered recoverable $(\mu \mathrm{g} / \mathrm{L})$ & 36 & 45.2 & 1.2 & 6.7 & 4.2 \\
Zinc, filtered $(\mu \mathrm{g} / \mathrm{L})$ & 36 & 5.6 & $<1.4$ & 21.1 & $<2.8$ \\
Zinc, unfiltered recoverable $(\mu \mathrm{g} / \mathrm{L})$ & 36 & 20.1 & $<2$ & 23.6 & 2.0 \\
Sediment, suspended $($ percent finer than 0.062 mm) & 36 & 83 & 32 & 64 & 66 \\
Sediment, suspended concentration $(\mathrm{mg} / \mathrm{L})$ & 36 & 65 & 1 & 9 & 4 \\
Sediment, suspended discharge $(\mathrm{ton} / \mathrm{d})$ & 68 & .13 & 5.3 & 1.3
\end{tabular}


Table 25. Statistical summary of long-term water-quality data for the Clark Fork basin, Montana, March 1985 through September 2011.-Continued

[Abbreviations: $\mathrm{CaCO}_{3}$, calcium carbonate; $\mathrm{ft} / \mathrm{s}$, cubic feet per second; ${ }^{\circ} \mathrm{C}$, degrees Celsius; $\mu \mathrm{g} / \mathrm{L}$, micrograms per liter; $\mu \mathrm{S} / \mathrm{cm}$, microsiemens per centimeter at $25^{\circ} \mathrm{C} ; \mathrm{mg} / \mathrm{L}$, milligrams per liter; $\mathrm{mm}$, millimeter; ton/d, tons per day. Symbols: $<$, less than laboratory reporting level $\left.{ }^{1}\right]$

\begin{tabular}{|c|c|c|c|c|c|}
\hline Property or constituent and reporting unit & $\begin{array}{l}\text { Number of } \\
\text { samples }\end{array}$ & Maximum & Minimum & Mean & Median \\
\hline \multicolumn{6}{|c|}{$\begin{array}{l}\text { 12323770--Warm Springs Creek at Warm Springs } \\
\text { eriod of record for water-quality data: March 1993-September } 2011\end{array}$} \\
\hline Streamflow, instantaneous $\left(\mathrm{ft}^{3} / \mathrm{s}\right)$ & 115 & 420 & 2.8 & 99 & 57 \\
\hline $\mathrm{pH}$, onsite (standard units) & 114 & 8.7 & 7.4 & 8.3 & 8.2 \\
\hline Specific conductance, onsite $(\mu \mathrm{S} / \mathrm{cm})$ & 114 & 795 & 139 & 291 & 296 \\
\hline Temperature, water $\left({ }^{\circ} \mathrm{C}\right)$ & 115 & 20.0 & 0.0 & 8.5 & 8.0 \\
\hline Hardness, filtered (mg/L as $\mathrm{CaCO}_{3}$ ) & 114 & 415 & 39.8 & 141 & 144 \\
\hline Calcium, filtered (mg/L) & 114 & 130 & 10.5 & 43.1 & 43.7 \\
\hline Magnesium, filtered (mg/L) & 114 & 22.0 & 3.29 & 8.13 & 8.06 \\
\hline Arsenic, filtered $(\mu \mathrm{g} / \mathrm{L})$ & 114 & 14.0 & 2.0 & 5.0 & 4.4 \\
\hline Arsenic, unfiltered recoverable $(\mu \mathrm{g} / \mathrm{L})$ & 114 & 27.0 & 3.0 & 7.4 & 6.0 \\
\hline Cadmium, filtered $(\mu \mathrm{g} / \mathrm{L})$ & 114 & .1 & $<.04$ & ${ }^{2} .04$ & $<.1$ \\
\hline Cadmium, unfiltered recoverable $(\mu \mathrm{g} / \mathrm{L})$ & 114 & .41 & $<.05$ & ${ }^{2} .08$ & .03 \\
\hline Copper, filtered ( $\mu \mathrm{g} / \mathrm{L})$ & 114 & 16.0 & 1.0 & 3.3 & 2.9 \\
\hline Copper, unfiltered recoverable $(\mu \mathrm{g} / \mathrm{L})$ & 114 & 147 & 2.3 & 19.6 & 9.0 \\
\hline Iron, filtered $(\mu \mathrm{g} / \mathrm{L})$ & 114 & 30 & $<5$ & ${ }^{2} 11$ & 10 \\
\hline Iron, unfiltered recoverable $(\mu \mathrm{g} / \mathrm{L})$ & 114 & 2,110 & 39 & 311 & 121 \\
\hline Lead, filtered $(\mu \mathrm{g} / \mathrm{L})$ & 114 & 1.8 & $<.08$ & ${ }^{2} .08$ & $<.6$ \\
\hline Lead, unfiltered recoverable $(\mu \mathrm{g} / \mathrm{L})$ & 114 & 14.0 & $<1.00$ & ${ }^{2} 1.92$ & .56 \\
\hline Manganese, filtered $(\mu \mathrm{g} / \mathrm{L})$ & 114 & 570 & 18.8 & 116 & 85.6 \\
\hline Manganese, unfiltered recoverable $(\mu \mathrm{g} / \mathrm{L})$ & 114 & 1,400 & 37.0 & 202 & 158 \\
\hline Zinc, filtered $(\mu \mathrm{g} / \mathrm{L})$ & 113 & 10 & $<1.0$ & ${ }^{2} 2.1$ & 1.4 \\
\hline Zinc, unfiltered recoverable $(\mu \mathrm{g} / \mathrm{L})$ & 114 & 60 & $<2.4$ & 29.2 & 3.0 \\
\hline Sediment, suspended (percent finer than $0.062 \mathrm{~mm}$ ) & 115 & 88 & 43 & 70 & 71 \\
\hline Sediment, suspended concentration (mg/L) & 115 & 127 & 1 & 18 & 8 \\
\hline Sediment, suspended discharge (ton/d) & 115 & 87 & .05 & 8.5 & 1.1 \\
\hline
\end{tabular}


Table 25. Statistical summary of long-term water-quality data for the Clark Fork basin, Montana, March 1985 through September 2011.-Continued

[Abbreviations: $\mathrm{CaCO}_{3}$, calcium carbonate; $\mathrm{ft} / \mathrm{s}$, cubic feet per second; ${ }^{\circ} \mathrm{C}$, degrees Celsius; $\mu \mathrm{g} / \mathrm{L}$, micrograms per liter; $\mu \mathrm{S} / \mathrm{cm}$, microsiemens per centimeter at $25^{\circ} \mathrm{C} ; \mathrm{mg} / \mathrm{L}$, milligrams per liter; $\mathrm{mm}$, millimeter; ton/d, tons per day. Symbols: $<$, less than laboratory reporting level $\left.{ }^{1}\right]$

\begin{tabular}{|c|c|c|c|c|c|}
\hline Property or constituent and reporting unit & $\begin{array}{l}\text { Number of } \\
\text { samples }\end{array}$ & Maximum & Minimum & Mean & Median \\
\hline \multicolumn{6}{|c|}{$\begin{array}{l}\text { 12323800--Clark Fork near Galen } \\
\text { eriod of record for water-quality data: July 1988-September } 2011\end{array}$} \\
\hline Streamflow, instantaneous $\left(\mathrm{ft}^{3} / \mathrm{s}\right)$ & 197 & 1,380 & 14 & 226 & 136 \\
\hline pH, onsite (standard units) & 184 & 9.2 & 7.5 & 8.5 & 8.6 \\
\hline Specific conductance, onsite $(\mu \mathrm{S} / \mathrm{cm})$ & 185 & 720 & 182 & 413 & 420 \\
\hline Temperature, water $\left({ }^{\circ} \mathrm{C}\right)$ & 196 & 23.5 & 0.0 & 9.8 & 10.0 \\
\hline Hardness, filtered (mg/L as $\left.\mathrm{CaCO}_{3}\right)$ & 183 & 365 & 76.4 & 180 & 188 \\
\hline Calcium, filtered (mg/L) & 183 & 110 & 23.2 & 53.4 & 55.3 \\
\hline Magnesium, filtered (mg/L) & 183 & 22.0 & 4.44 & 11.4 & 11.8 \\
\hline Arsenic, filtered $(\mu \mathrm{g} / \mathrm{L})$ & 183 & 53.0 & 4.0 & 15.2 & 14.8 \\
\hline Arsenic, unfiltered recoverable $(\mu \mathrm{g} / \mathrm{L})$ & 183 & 78.0 & 3.0 & 19.5 & 17.0 \\
\hline Cadmium, filtered $(\mu \mathrm{g} / \mathrm{L})$ & 183 & 1.0 & $<.04$ & ${ }^{2} .06$ & $<1$ \\
\hline Cadmium, unfiltered recoverable $(\mu \mathrm{g} / \mathrm{L})$ & 183 & 3.0 & $<.1$ & ${ }^{2} .19$ & .05 \\
\hline Copper, filtered $(\mu \mathrm{g} / \mathrm{L})$ & 183 & 50.0 & 1.7 & 7.8 & 6.0 \\
\hline Copper, unfiltered recoverable ( $\mu \mathrm{g} / \mathrm{L})$ & 182 & 240 & 4.1 & 27.5 & 15.5 \\
\hline Iron, filtered $(\mu \mathrm{g} / \mathrm{L})$ & 183 & 110 & $<3$ & ${ }^{2} 16$ & 11 \\
\hline Iron, unfiltered recoverable $(\mu \mathrm{g} / \mathrm{L})$ & 183 & 9,200 & 56 & 477 & 270 \\
\hline Lead, filtered $(\mu \mathrm{g} / \mathrm{L})$ & 183 & 3.00 & $<.08$ & ${ }^{2} .15$ & $<1.00$ \\
\hline Lead, unfiltered recoverable $(\mu \mathrm{g} / \mathrm{L})$ & 183 & 31.0 & $<1.00$ & 23.42 & 1.86 \\
\hline Manganese, filtered $(\mu \mathrm{g} / \mathrm{L})$ & 183 & 460 & 24.0 & 105 & 76.0 \\
\hline Manganese, unfiltered recoverable $(\mu \mathrm{g} / \mathrm{L})$ & 183 & 1,400 & 47.3 & 221 & 170 \\
\hline Zinc, filtered $(\mu \mathrm{g} / \mathrm{L})$ & 183 & 110 & $<1.0$ & ${ }^{2} 8.9$ & 4.4 \\
\hline Zinc, unfiltered recoverable $(\mu \mathrm{g} / \mathrm{L})$ & 183 & 360 & $<10.0$ & 236.4 & 20.0 \\
\hline Sediment, suspended (percent finer than $0.062 \mathrm{~mm}$ ) & 196 & 97 & 32 & 76 & 77 \\
\hline Sediment, suspended concentration (mg/L) & 197 & 338 & 1 & 18 & 8 \\
\hline Sediment, suspended discharge (ton/d) & 197 & 459 & .12 & 21 & 2.8 \\
\hline
\end{tabular}


Table 25. Statistical summary of long-term water-quality data for the Clark Fork basin, Montana, March 1985 through September 2011.-Continued

[Abbreviations: $\mathrm{CaCO}_{3}$, calcium carbonate; $\mathrm{ft} / \mathrm{s}$, cubic feet per second; ${ }^{\circ} \mathrm{C}$, degrees Celsius; $\mu \mathrm{g} / \mathrm{L}$, micrograms per liter; $\mu \mathrm{S} / \mathrm{cm}$, microsiemens per centimeter at $25^{\circ} \mathrm{C} ; \mathrm{mg} / \mathrm{L}$, milligrams per liter; $\mathrm{mm}$, millimeter; ton/d, tons per day. Symbols: $<$, less than laboratory reporting level $\left.{ }^{1}\right]$

\begin{tabular}{|c|c|c|c|c|c|}
\hline Property or constituent and reporting unit & $\begin{array}{l}\text { Number of } \\
\text { samples }\end{array}$ & Maximum & Minimum & Mean & Median \\
\hline \multicolumn{6}{|c|}{$\begin{array}{l}\text { 12323840--Lost Creek near Anaconda } \\
\text { Period of record for water-quality data: December 2004-September } 2011\end{array}$} \\
\hline Streamflow, instantaneous $\left(\mathrm{ft}^{3} / \mathrm{s}\right)$ & 55 & 73 & 0.37 & 13 & 8.2 \\
\hline $\mathrm{pH}$, onsite (standard units) & 55 & 8.6 & 7.4 & 8.2 & 8.2 \\
\hline Specific conductance, onsite $(\mu \mathrm{S} / \mathrm{cm})$ & 55 & 253 & 121 & 197 & 210 \\
\hline Temperature, water $\left({ }^{\circ} \mathrm{C}\right)$ & 55 & 17.0 & 1.0 & 8.0 & 8.0 \\
\hline Hardness, filtered $\left(\mathrm{mg} / \mathrm{L}\right.$ as $\left.\mathrm{CaCO}_{3}\right)$ & 55 & 122 & 50.4 & 93.3 & 98.6 \\
\hline Calcium, filtered $(\mathrm{mg} / \mathrm{L})$ & 55 & 37.1 & 15.7 & 28.3 & 29.3 \\
\hline Magnesium, filtered (mg/L) & 55 & 7.22 & 2.71 & 5.47 & 5.78 \\
\hline Arsenic, filtered $(\mu \mathrm{g} / \mathrm{L})$ & 55 & 156 & 1.8 & 7.2 & 3.2 \\
\hline Arsenic, unfiltered recoverable $(\mu \mathrm{g} / \mathrm{L})$ & 55 & 3,860 & 2.0 & 75.2 & 3.8 \\
\hline Cadmium, filtered $(\mu \mathrm{g} / \mathrm{L})$ & 54 & .90 & $<.02$ & ${ }^{2} .04$ & .02 \\
\hline Cadmium, unfiltered recoverable $(\mu \mathrm{g} / \mathrm{L})$ & 55 & 147 & .01 & 22.7 & .04 \\
\hline Copper, filtered $(\mu \mathrm{g} / \mathrm{L})$ & 55 & 90.5 & .80 & 3.6 & 1.7 \\
\hline Copper, unfiltered recoverable $(\mu \mathrm{g} / \mathrm{L})$ & 55 & 29,100 & 1.3 & 536 & 4.5 \\
\hline Iron, filtered $(\mu \mathrm{g} / \mathrm{L})$ & 55 & 27 & $<6$ & ${ }^{2} 10$ & 9 \\
\hline Iron, unfiltered recoverable $(\mu \mathrm{g} / \mathrm{L})$ & 55 & 99,700 & 22 & 2,020 & 110 \\
\hline Lead, filtered $(\mu \mathrm{g} / \mathrm{L})$ & 55 & .18 & $<.03$ & ${ }^{2} .04$ & .02 \\
\hline Lead, unfiltered recoverable $(\mu \mathrm{g} / \mathrm{L})$ & 55 & 1,290 & .08 & 24.4 & .46 \\
\hline Manganese, filtered $(\mu \mathrm{g} / \mathrm{L})$ & 55 & 42.4 & $<.2$ & 22.0 & 1.1 \\
\hline Manganese, unfiltered recoverable $(\mu \mathrm{g} / \mathrm{L})$ & 55 & 8,830 & 1.2 & 168 & 4.6 \\
\hline Zinc, filtered $(\mu \mathrm{g} / \mathrm{L})$ & 54 & 30.0 & $<1.4$ & ${ }^{2} 1.8$ & 1.0 \\
\hline Zinc, unfiltered recoverable $(\mu \mathrm{g} / \mathrm{L})$ & 54 & 7,780 & $<2$ & ${ }^{2} 148$ & 2.5 \\
\hline Sediment, suspended (percent finer than $0.062 \mathrm{~mm}$ ) & 55 & 97 & 22 & 58 & 59 \\
\hline Sediment, suspended concentration (mg/L) & 55 & 58,900 & 1 & 1,080 & 6 \\
\hline Sediment, suspended discharge (ton/d) & 55 & 1,320 & $<.01$ & ${ }^{2} 25$ & .11 \\
\hline
\end{tabular}


Table 25. Statistical summary of long-term water-quality data for the Clark Fork basin, Montana, March 1985 through September 2011.-Continued

[Abbreviations: $\mathrm{CaCO}_{3}$, calcium carbonate; $\mathrm{ft} / \mathrm{s}$, cubic feet per second; ${ }^{\circ} \mathrm{C}$, degrees Celsius; $\mu \mathrm{g} / \mathrm{L}$, micrograms per liter; $\mu \mathrm{S} / \mathrm{cm}$, microsiemens per centimeter at $25^{\circ} \mathrm{C} ; \mathrm{mg} / \mathrm{L}$, milligrams per liter; $\mathrm{mm}$, millimeter; ton/d, tons per day. Symbols: <, less than laboratory reporting level $\left.{ }^{1}\right]$

\begin{tabular}{|c|c|c|c|c|c|}
\hline Property or constituent and reporting unit & $\begin{array}{l}\text { Number of } \\
\text { samples }\end{array}$ & Maximum & Minimum & Mean & Median \\
\hline \multicolumn{6}{|c|}{$\begin{array}{l}\text { 12323850--Lost Creek near Galen } \\
\text { riod of record for water-quality data: March 2003-September } 2011\end{array}$} \\
\hline Streamflow, instantaneous $\left(\mathrm{ft}^{3} / \mathrm{s}\right)$ & 72 & 71 & 1.3 & 23 & 16 \\
\hline pH, onsite (standard units) & 72 & 8.7 & 8.0 & 8.3 & 8.3 \\
\hline Specific conductance, onsite $(\mu \mathrm{S} / \mathrm{cm})$ & 72 & 934 & 540 & 645 & 630 \\
\hline Temperature, water $\left({ }^{\circ} \mathrm{C}\right)$ & 72 & 26.5 & 0.0 & 10.5 & 9.8 \\
\hline Hardness, filtered $\left(\mathrm{mg} / \mathrm{L}\right.$ as $\left.\mathrm{CaCO}_{3}\right)$ & 72 & 451 & 203 & 301 & 299 \\
\hline Calcium, filtered (mg/L) & 72 & 122 & 48.5 & 85.1 & 85.3 \\
\hline Magnesium, filtered (mg/L) & 72 & 35.7 & 17.3 & 21.6 & 20.8 \\
\hline Arsenic, filtered $(\mu \mathrm{g} / \mathrm{L})$ & 72 & 41.8 & 6.0 & 14.4 & 12.6 \\
\hline Arsenic, unfiltered recoverable $(\mu \mathrm{g} / \mathrm{L})$ & 72 & 43.0 & 6.0 & 15.2 & 14.0 \\
\hline Cadmium, filtered $(\mu \mathrm{g} / \mathrm{L})$ & 71 & .05 & $<.04$ & ${ }^{2} .03$ & .02 \\
\hline Cadmium, unfiltered recoverable $(\mu \mathrm{g} / \mathrm{L})$ & 72 & .11 & .01 & ${ }^{2} .04$ & .04 \\
\hline Copper, filtered $(\mu \mathrm{g} / \mathrm{L})$ & 72 & 6.7 & .99 & 2.3 & 2.2 \\
\hline Copper, unfiltered recoverable $(\mu \mathrm{g} / \mathrm{L})$ & 72 & 22.5 & 1.6 & 5.4 & 4.4 \\
\hline Iron, filtered $(\mu \mathrm{g} / \mathrm{L})$ & 72 & 61 & $<6$ & ${ }^{2} 13$ & 10 \\
\hline Iron, unfiltered recoverable $(\mu \mathrm{g} / \mathrm{L})$ & 72 & 293 & 14 & 99 & 78 \\
\hline Lead, filtered $(\mu \mathrm{g} / \mathrm{L})$ & 71 & .33 & $<.06$ & ${ }^{2} .05$ & $<.02$ \\
\hline Lead, unfiltered recoverable $(\mu \mathrm{g} / \mathrm{L})$ & 72 & 1.30 & .04 & .35 & .26 \\
\hline Manganese, filtered $(\mu \mathrm{g} / \mathrm{L})$ & 72 & 54.0 & 1.9 & 15.3 & 13.6 \\
\hline Manganese, unfiltered recoverable $(\mu \mathrm{g} / \mathrm{L})$ & 72 & 56.5 & 2.2 & 20.5 & 18.4 \\
\hline Zinc, filtered $(\mu \mathrm{g} / \mathrm{L})$ & 71 & 3.8 & $<1.0$ & ${ }^{2} 1.5$ & 1.3 \\
\hline Zinc, unfiltered recoverable $(\mu \mathrm{g} / \mathrm{L})$ & 72 & 9.0 & $<2$ & 22.8 & 2.0 \\
\hline Sediment, suspended (percent finer than $0.062 \mathrm{~mm}$ ) & 72 & 86 & 18 & 58 & 60 \\
\hline Sediment, suspended concentration (mg/L) & 72 & 79 & 2 & 16 & 15 \\
\hline Sediment, suspended discharge (ton/d) & 72 & 4.2 & .01 & 1.0 & .52 \\
\hline
\end{tabular}


Table 25. Statistical summary of long-term water-quality data for the Clark Fork basin, Montana, March 1985 through September 2011.-Continued

[Abbreviations: $\mathrm{CaCO}_{3}$, calcium carbonate; $\mathrm{ft} / \mathrm{s}$, cubic feet per second; ${ }^{\circ} \mathrm{C}$, degrees Celsius; $\mu \mathrm{g} / \mathrm{L}$, micrograms per liter; $\mu \mathrm{S} / \mathrm{cm}$, microsiemens per centimeter at $25^{\circ} \mathrm{C} ; \mathrm{mg} / \mathrm{L}$, milligrams per liter; $\mathrm{mm}$, millimeter; ton/d, tons per day. Symbols: $<$, less than laboratory reporting level $\left.{ }^{1}\right]$

\begin{tabular}{|c|c|c|c|c|c|}
\hline Property or constituent and reporting unit & $\begin{array}{l}\text { Number of } \\
\text { samples }\end{array}$ & Maximum & Minimum & Mean & Median \\
\hline \multicolumn{6}{|c|}{$\begin{array}{l}\text { 12324200--Clark Fork at Deer Lodge } \\
\text { ariod of record for water-quality data: March 1985-September } 2011\end{array}$} \\
\hline Streamflow, instantaneous $\left(\mathrm{ft}^{3} / \mathrm{s}\right)$ & 249 & 2,000 & 23 & 310 & 227 \\
\hline $\mathrm{pH}$, onsite (standard units) & 197 & 8.9 & 7.4 & 8.3 & 8.3 \\
\hline Specific conductance, onsite $(\mu \mathrm{S} / \mathrm{cm})$ & 232 & 642 & 228 & 471 & 498 \\
\hline Temperature, water $\left({ }^{\circ} \mathrm{C}\right)$ & 248 & 23.0 & 0.0 & 9.9 & 10.0 \\
\hline Hardness, filtered (mg/L as $\mathrm{CaCO}_{3}$ ) & 189 & 282 & 94.9 & 199 & 208 \\
\hline Calcium, filtered (mg/L) & 189 & 82.0 & 28.2 & 58.8 & 61.0 \\
\hline Magnesium, filtered (mg/L) & 189 & 18.7 & 5.53 & 12.6 & 13.2 \\
\hline Arsenic, filtered $(\mu \mathrm{g} / \mathrm{L})$ & 199 & 39.0 & 6.0 & 14.6 & 13.7 \\
\hline Arsenic, unfiltered recoverable $(\mu \mathrm{g} / \mathrm{L})$ & 198 & 215 & 4.8 & 24.0 & 18.0 \\
\hline Cadmium, filtered $(\mu \mathrm{g} / \mathrm{L})$ & 198 & 2.0 & $<.10$ & ${ }^{2} .08$ & $<1.0$ \\
\hline Cadmium, unfiltered recoverable $(\mu \mathrm{g} / \mathrm{L})$ & 198 & 5.0 & $<.10$ & ${ }^{2} .40$ & .10 \\
\hline Copper, filtered ( $\mu \mathrm{g} / \mathrm{L})$ & 199 & 120 & 3.2 & 10.9 & 8.0 \\
\hline Copper, unfiltered recoverable $(\mu \mathrm{g} / \mathrm{L})$ & 197 & 1,500 & 8.2 & 80.2 & 37.0 \\
\hline Iron, filtered $(\mu \mathrm{g} / \mathrm{L})$ & 199 & 190 & $<3$ & ${ }^{2} 15$ & 9 \\
\hline Iron, unfiltered recoverable $(\mu \mathrm{g} / \mathrm{L})$ & 199 & 29,000 & 27 & 1,450 & 520 \\
\hline Lead, filtered $(\mu \mathrm{g} / \mathrm{L})$ & 199 & 6.00 & $<.08$ & ${ }^{2} .31$ & $<1.00$ \\
\hline Lead, unfiltered recoverable $(\mu \mathrm{g} / \mathrm{L})$ & 199 & 200 & .33 & ${ }^{2} 10.6$ & 4.70 \\
\hline Manganese, filtered $(\mu \mathrm{g} / \mathrm{L})$ & 199 & 400 & 1.0 & 41.3 & 33.4 \\
\hline Manganese, unfiltered recoverable $(\mu \mathrm{g} / \mathrm{L})$ & 199 & 4,600 & 11.9 & 231 & 132 \\
\hline Zinc, filtered $(\mu \mathrm{g} / \mathrm{L})$ & 199 & 230 & $<10.0$ & ${ }^{2} 11.7$ & 8.0 \\
\hline Zinc, unfiltered recoverable $(\mu \mathrm{g} / \mathrm{L})$ & 197 & 1,700 & 4 & 84.2 & 40.0 \\
\hline Sediment, suspended (percent finer than $0.062 \mathrm{~mm}$ ) & 240 & 99 & 31 & 71 & 72 \\
\hline Sediment, suspended concentration (mg/L) & 249 & 2,250 & 1 & 69 & 22 \\
\hline Sediment, suspended discharge (ton/d) & 249 & 8,690 & .18 & 145 & 12 \\
\hline
\end{tabular}


Table 25. Statistical summary of long-term water-quality data for the Clark Fork basin, Montana, March 1985 through September 2011.-Continued

[Abbreviations: $\mathrm{CaCO}_{3}$, calcium carbonate; $\mathrm{ft} / \mathrm{s}$, cubic feet per second; ${ }^{\circ} \mathrm{C}$, degrees Celsius; $\mu \mathrm{g} / \mathrm{L}$, micrograms per liter; $\mu \mathrm{S} / \mathrm{cm}$, microsiemens per centimeter at $25^{\circ} \mathrm{C} ; \mathrm{mg} / \mathrm{L}$, milligrams per liter; $\mathrm{mm}$, millimeter; ton/d, tons per day. Symbols: <, less than laboratory reporting level $\left.{ }^{1}\right]$

\begin{tabular}{|c|c|c|c|c|c|}
\hline Property or constituent and reporting unit & $\begin{array}{l}\text { Number of } \\
\text { samples }\end{array}$ & Maximum & Minimum & Mean & Median \\
\hline \multicolumn{6}{|c|}{$\begin{array}{l}\text { 12324400--Clark Fork above Little Blackfoot River, near Garrison } \\
\text { Period of record for water-quality data: March 2009-September } 2011\end{array}$} \\
\hline Streamflow, instantaneous $\left(\mathrm{ft}^{3} / \mathrm{s}\right)$ & 23 & 2,310 & 186 & 626 & 353 \\
\hline pH, onsite (standard units) & 23 & 8.8 & 7.9 & 8.3 & 8.3 \\
\hline Specific conductance, onsite $(\mu \mathrm{S} / \mathrm{cm})$ & 23 & 494 & 249 & 390 & 386 \\
\hline Temperature, water $\left({ }^{\circ} \mathrm{C}\right)$ & 23 & 19.0 & 3.0 & 10.7 & 12.0 \\
\hline Hardness, filtered (mg/L as $\mathrm{CaCO}_{3}$ ) & 23 & 221 & 104 & 169 & 160 \\
\hline Calcium, filtered $(\mathrm{mg} / \mathrm{L})$ & 23 & 64.0 & 31.8 & 49.8 & 46.9 \\
\hline Magnesium, filtered (mg/L) & 23 & 14.8 & 5.93 & 10.8 & 10.4 \\
\hline Arsenic, filtered $(\mu \mathrm{g} / \mathrm{L})$ & 23 & 36.7 & 9.2 & 16.3 & 16.4 \\
\hline Arsenic, unfiltered recoverable $(\mu \mathrm{g} / \mathrm{L})$ & 23 & 46.0 & 11.7 & 24.8 & 20.7 \\
\hline Cadmium, filtered $(\mu \mathrm{g} / \mathrm{L})$ & 23 & .23 & .04 & .08 & .08 \\
\hline Cadmium, unfiltered recoverable $(\mu \mathrm{g} / \mathrm{L})$ & 23 & .75 & .08 & .34 & .25 \\
\hline Copper, filtered $(\mu \mathrm{g} / \mathrm{L})$ & 23 & 40.6 & 4.2 & 11.4 & 9.1 \\
\hline Copper, unfiltered recoverable $(\mu \mathrm{g} / \mathrm{L})$ & 23 & 197 & 12.4 & 76.3 & 59.4 \\
\hline Iron, filtered $(\mu \mathrm{g} / \mathrm{L})$ & 23 & 43 & 5 & 16 & 11 \\
\hline Iron, unfiltered recoverable $(\mu \mathrm{g} / \mathrm{L})$ & 23 & 3,200 & 119 & 1,140 & 731 \\
\hline Lead, filtered $(\mu \mathrm{g} / \mathrm{L})$ & 23 & .39 & .04 & .15 & .11 \\
\hline Lead, unfiltered recoverable ( $\mu \mathrm{g} / \mathrm{L})$ & 23 & 29.4 & .90 & 10.2 & 7.75 \\
\hline Manganese, filtered $(\mu \mathrm{g} / \mathrm{L})$ & 23 & 45.4 & 15.6 & 27.3 & 23.6 \\
\hline Manganese, unfiltered recoverable $(\mu \mathrm{g} / \mathrm{L})$ & 23 & 309 & 40.5 & 144 & 129 \\
\hline Zinc, filtered $(\mu \mathrm{g} / \mathrm{L})$ & 23 & 37.1 & 2.9 & 7.6 & 6.0 \\
\hline Zinc, unfiltered recoverable $(\mu \mathrm{g} / \mathrm{L})$ & 23 & 152 & 10.1 & 62.9 & 41.2 \\
\hline Sediment, suspended (percent finer than $0.062 \mathrm{~mm}$ ) & 23 & 85 & 42 & 66 & 66 \\
\hline Sediment, suspended concentration (mg/L) & 23 & 179 & 5 & 59 & 30 \\
\hline Sediment, suspended discharge (ton/d) & 23 & 428 & 2.5 & 131 & 42 \\
\hline
\end{tabular}


Table 25. Statistical summary of long-term water-quality data for the Clark Fork basin, Montana, March 1985 through September 2011.-Continued

[Abbreviations: $\mathrm{CaCO}_{3}$, calcium carbonate; $\mathrm{ft} / \mathrm{s}$, cubic feet per second; ${ }^{\circ} \mathrm{C}$, degrees Celsius; $\mu \mathrm{g} / \mathrm{L}$, micrograms per liter; $\mu \mathrm{S} / \mathrm{cm}$, microsiemens per centimeter at $25^{\circ} \mathrm{C} ; \mathrm{mg} / \mathrm{L}$, milligrams per liter; $\mathrm{mm}$, millimeter; ton/d, tons per day. Symbols: <, less than laboratory reporting level $\left.{ }^{1}\right]$

\begin{tabular}{|c|c|c|c|c|c|}
\hline Property or constituent and reporting unit & $\begin{array}{l}\text { Number of } \\
\text { samples }\end{array}$ & Maximum & Minimum & Mean & Median \\
\hline \multicolumn{6}{|c|}{$\begin{array}{l}\text { 12324680--Clark Fork at Goldcreek } \\
\text { eriod of record for water-quality data: March 1993-September } 2011\end{array}$} \\
\hline Streamflow, instantaneous $\left(\mathrm{ft}^{3} / \mathrm{s}\right)$ & 155 & 4,450 & 87 & 799 & 529 \\
\hline $\mathrm{pH}$, onsite (standard units) & 154 & 8.9 & 7.9 & 8.4 & 8.3 \\
\hline Specific conductance, onsite $(\mu \mathrm{S} / \mathrm{cm})$ & 154 & 510 & 206 & 367 & 380 \\
\hline Temperature, water $\left({ }^{\circ} \mathrm{C}\right)$ & 155 & 23.0 & 0.0 & 10.0 & 10.5 \\
\hline Hardness, filtered (mg/L as $\mathrm{CaCO}_{3}$ ) & 154 & 232 & 85.8 & 161 & 169 \\
\hline Calcium, filtered (mg/L) & 154 & 68.0 & 25.9 & 47.5 & 49.7 \\
\hline Magnesium, filtered (mg/L) & 154 & 15.0 & 5.15 & 10.3 & 10.6 \\
\hline Arsenic, filtered $(\mu \mathrm{g} / \mathrm{L})$ & 154 & 22.5 & 5.8 & 10.2 & 10.0 \\
\hline Arsenic, unfiltered recoverable $(\mu \mathrm{g} / \mathrm{L})$ & 154 & 75.0 & 7.0 & 15.0 & 12.6 \\
\hline Cadmium, filtered $(\mu \mathrm{g} / \mathrm{L})$ & 154 & .2 & $<.04$ & ${ }^{2} .04$ & $<.10$ \\
\hline Cadmium, unfiltered recoverable $(\mu \mathrm{g} / \mathrm{L})$ & 154 & 2 & $<.10$ & ${ }^{2} .18$ & .06 \\
\hline Copper, filtered $(\mu \mathrm{g} / \mathrm{L})$ & 153 & 36.0 & 2.1 & 6.8 & 5.4 \\
\hline Copper, unfiltered recoverable $(\mu \mathrm{g} / \mathrm{L})$ & 153 & 440 & 5.2 & 40.4 & 24.5 \\
\hline Iron, filtered $(\mu \mathrm{g} / \mathrm{L})$ & 154 & 100 & $<3$ & ${ }^{2} 19$ & 12 \\
\hline Iron, unfiltered recoverable $(\mu \mathrm{g} / \mathrm{L})$ & 154 & 12,000 & 27 & 868 & 433 \\
\hline Lead, filtered $(\mu \mathrm{g} / \mathrm{L})$ & 152 & .6 & $<.08$ & ${ }^{2} .11$ & $<.6$ \\
\hline Lead, unfiltered recoverable $(\mu \mathrm{g} / \mathrm{L})$ & 153 & 73.0 & .14 & ${ }^{2} 5.64$ & 3.00 \\
\hline Manganese, filtered $(\mu \mathrm{g} / \mathrm{L})$ & 154 & 57.3 & 4.0 & 18.9 & 17.0 \\
\hline Manganese, unfiltered recoverable $(\mu \mathrm{g} / \mathrm{L})$ & 154 & 1,100 & 10.5 & 120 & 87.7 \\
\hline Zinc, filtered $(\mu \mathrm{g} / \mathrm{L})$ & 154 & 26 & $<1.0$ & ${ }^{2} 5.6$ & 4.0 \\
\hline Zinc, unfiltered recoverable $(\mu \mathrm{g} / \mathrm{L})$ & 154 & 510 & 2 & 43.7 & 30.0 \\
\hline Sediment, suspended (percent finer than $0.062 \mathrm{~mm}$ ) & 155 & 94 & 43 & 74 & 77 \\
\hline Sediment, suspended concentration (mg/L) & 155 & 752 & 1 & 49 & 22 \\
\hline Sediment, suspended discharge (ton/d) & 155 & 7,960 & .55 & 215 & 33 \\
\hline
\end{tabular}


Table 25. Statistical summary of long-term water-quality data for the Clark Fork basin, Montana, March 1985 through September 2011.-Continued

[Abbreviations: $\mathrm{CaCO}_{3}$, calcium carbonate; $\mathrm{ft} / \mathrm{s}$, cubic feet per second; ${ }^{\circ} \mathrm{C}$, degrees Celsius; $\mu \mathrm{g} / \mathrm{L}$, micrograms per liter; $\mu \mathrm{S} / \mathrm{cm}$, microsiemens per centimeter at $25^{\circ} \mathrm{C} ; \mathrm{mg} / \mathrm{L}$, milligrams per liter; $\mathrm{mm}$, millimeter; ton/d, tons per day. Symbols: $<$, less than laboratory reporting level $\left.{ }^{1}\right]$

\begin{tabular}{lccccc}
\hline \multicolumn{1}{c}{ Property or constituent and reporting unit } & $\begin{array}{c}\text { Number of } \\
\text { samples }\end{array}$ & Maximum & Minimum & Mean & Median \\
\hline \multicolumn{1}{c}{ Period of record for water-quality data: March 1993-September 2011 } & & \\
\hline Streamflow, instantaneous $(\mathrm{ft} / \mathrm{s})$ & 155 & 5,540 & 149 & 1,100 & 799 \\
$\mathrm{pH}$, onsite (standard units) & 154 & 8.7 & 7.8 & 8.3 & 8.3 \\
Specific conductance, onsite $(\mu \mathrm{S} / \mathrm{cm})$ & 154 & 630 & 189 & 405 & 417 \\
Temperature, water $\left({ }^{\circ} \mathrm{C}\right)$ & 155 & 22.5 & .5 & 10.9 & 11.0 \\
Hardness, filtered $\left(\mathrm{mg} / \mathrm{L}\right.$ as $\left.\mathrm{CaCO}_{3}\right)$ & 154 & 298 & 73.9 & 182 & 189 \\
Calcium, filtered $(\mathrm{mg} / \mathrm{L})$ & 154 & 83.0 & 21.0 & 52.2 & 54.3 \\
Magnesium, filtered $(\mathrm{mg} / \mathrm{L})$ & 154 & 22.0 & 5.2 & 12.5 & 12.8 \\
Arsenic, filtered $(\mu \mathrm{g} / \mathrm{L})$ & 154 & 23.9 & 3.2 & 10.6 & 10.0 \\
Arsenic, unfiltered recoverable $(\mu \mathrm{g} / \mathrm{L})$ & 154 & 62 & 8 & 16.3 & 13.0 \\
Cadmium, filtered $(\mu \mathrm{g} / \mathrm{L})$ & 153 & .30 & $<.04$ & 2.05 & .02 \\
Cadmium, unfiltered recoverable $(\mu \mathrm{g} / \mathrm{L})$ & 154 & 2.0 & $<.10$ & 2.24 & .08 \\
Copper, filtered $(\mu \mathrm{g} / \mathrm{L})$ & 151 & 21.0 & 1.0 & 6.6 & 5.0 \\
Copper, unfiltered recoverable $(\mu \mathrm{g} / \mathrm{L})$ & 152 & 360 & 4.6 & 43.3 & 23.0 \\
Iron, filtered $(\mu \mathrm{g} / \mathrm{L})$ & 154 & 150 & $<3$ & 219 & 9 \\
Iron, unfiltered recoverable $(\mu \mathrm{g} / \mathrm{L})$ & 153 & 8,800 & 20 & 1,010 & 490 \\
Lead, filtered $(\mu \mathrm{g} / \mathrm{L})$ & 150 & 1.2 & $<.08$ & 2.17 & $<1.0$ \\
Lead, unfiltered recoverable $(\mu \mathrm{g} / \mathrm{L})$ & 150 & 56.0 & $<1.00$ & 27.76 & 3.61 \\
Manganese, filtered $(\mu \mathrm{g} / \mathrm{L})$ & 153 & 60.7 & 3.3 & 16.5 & 14.4 \\
Manganese, unfiltered recoverable $(\mu \mathrm{g} / \mathrm{L})$ & 154 & 880 & 8.0 & 146 & 96.0 \\
Zinc, filtered $(\mu \mathrm{g} / \mathrm{L})$ & 154 & 21.0 & $<3$ & 26.0 & 4.4 \\
Zinc, unfiltered recoverable $(\mu \mathrm{g} / \mathrm{L})$ & 154 & 490 & 2.9 & 60.0 & 31.9 \\
Sediment, suspended $($ percent finer than 0.062 mm) & 155 & 92 & 38 & 73 & 74 \\
Sediment, suspended concentration $(\mathrm{mg} / \mathrm{L})$ & 155 & 530 & 2 & 64 & 27 \\
Sediment, suspended discharge $(\mathrm{ton} / \mathrm{d})$ & 155 & 4,720 & 1.7 & 333 & 53
\end{tabular}


Table 25. Statistical summary of long-term water-quality data for the Clark Fork basin, Montana, March 1985 through September 2011.-Continued

[Abbreviations: $\mathrm{CaCO}_{3}$, calcium carbonate; $\mathrm{ft}^{3} / \mathrm{s}$, cubic feet per second; ${ }^{\circ} \mathrm{C}$, degrees Celsius; $\mu \mathrm{g} / \mathrm{L}$, micrograms per liter; $\mu \mathrm{S} / \mathrm{cm}$, microsiemens per centimeter at $25^{\circ} \mathrm{C} ; \mathrm{mg} / \mathrm{L}$, milligrams per liter; $\mathrm{mm}$, millimeter; ton/d, tons per day. Symbols: $<$, less than laboratory reporting level $\left.{ }^{1}\right]$

\begin{tabular}{|c|c|c|c|c|c|}
\hline Property or constituent and reporting unit & $\begin{array}{l}\text { Number of } \\
\text { samples }\end{array}$ & Maximum & Minimum & Mean & Median \\
\hline \multicolumn{6}{|c|}{$\begin{array}{l}\text { 12334550--Clark Fork at Turah Bridge, near Bonner } \\
\text { d of record for water-quality data: March 1985-September } 2011\end{array}$} \\
\hline Streamflow, instantaneous $\left(\mathrm{ft}^{3} / \mathrm{s}\right)$ & 252 & 10,600 & 296 & 1,980 & 1,160 \\
\hline pH, onsite (standard units) & 198 & 8.8 & 7.4 & 8.2 & 8.2 \\
\hline Specific conductance, onsite $(\mu \mathrm{S} / \mathrm{cm})$ & 227 & 483 & 139 & 299 & 313 \\
\hline Temperature, water $\left({ }^{\circ} \mathrm{C}\right)$ & 251 & 22.0 & 0.0 & 9.5 & 10.0 \\
\hline Hardness, filtered (mg/L as $\left.\mathrm{CaCO}_{3}\right)$ & 188 & 205 & 53.6 & 130 & 132 \\
\hline Calcium, filtered (mg/L) & 188 & 59.0 & 14.9 & 36.8 & 37.2 \\
\hline Magnesium, filtered (mg/L) & 188 & 14.0 & 3.94 & 9.36 & 9.39 \\
\hline Arsenic, filtered $(\mu \mathrm{g} / \mathrm{L})$ & 197 & 17.0 & 2.7 & 6.1 & 5.6 \\
\hline Arsenic, unfiltered recoverable $(\mu \mathrm{g} / \mathrm{L})$ & 197 & 110 & 3.0 & 9.8 & 7.0 \\
\hline Cadmium, filtered $(\mu \mathrm{g} / \mathrm{L})$ & 196 & .10 & $<.04$ & ${ }^{2} .04$ & $<.1$ \\
\hline Cadmium, unfiltered recoverable $(\mu \mathrm{g} / \mathrm{L})$ & 197 & 4.00 & $<.01$ & 2.25 & $<1.00$ \\
\hline Copper, filtered $(\mu \mathrm{g} / \mathrm{L})$ & 196 & 25.0 & 1.1 & 4.8 & 3.9 \\
\hline Copper, unfiltered recoverable $(\mu \mathrm{g} / \mathrm{L})$ & 195 & 500 & 2.7 & 33.8 & 16.0 \\
\hline Iron, filtered $(\mu \mathrm{g} / \mathrm{L})$ & 197 & 190 & $<3$ & 224 & 13 \\
\hline Iron, unfiltered recoverable $(\mu \mathrm{g} / \mathrm{L})$ & 197 & 19,000 & 33 & 1,000 & 389 \\
\hline Lead, filtered $(\mu \mathrm{g} / \mathrm{L})$ & 193 & 7.00 & $<.08$ & 2.28 & $<1.00$ \\
\hline Lead, unfiltered recoverable $(\mu \mathrm{g} / \mathrm{L})$ & 193 & 100 & $<1.00$ & ${ }^{2} 6.87$ & 3.00 \\
\hline Manganese, filtered $(\mu \mathrm{g} / \mathrm{L})$ & 197 & 37.4 & $<1.0$ & ${ }^{2} 8.4$ & 7.0 \\
\hline Manganese, unfiltered recoverable $(\mu \mathrm{g} / \mathrm{L})$ & 197 & 2,000 & 8.9 & 120 & 62.8 \\
\hline Zinc, filtered $(\mu \mathrm{g} / \mathrm{L})$ & 195 & 39 & $<3.0$ & ${ }^{2} 5.9$ & 4.0 \\
\hline Zinc, unfiltered recoverable $(\mu \mathrm{g} / \mathrm{L})$ & 197 & 1,100 & $<10.0$ & 257.2 & 29.0 \\
\hline Sediment, suspended (percent finer than $0.062 \mathrm{~mm}$ ) & 241 & 98 & 27 & 73 & 75 \\
\hline Sediment, suspended concentration (mg/L) & 252 & 1,370 & 2 & 56 & 18 \\
\hline Sediment, suspended discharge (ton/d) & 252 & 34,700 & 3.0 & 640 & 61 \\
\hline
\end{tabular}


Table 25. Statistical summary of long-term water-quality data for the Clark Fork basin, Montana, March 1985 through September 2011.-Continued

[Abbreviations: $\mathrm{CaCO}_{3}$, calcium carbonate; $\mathrm{ft} / \mathrm{s}$, cubic feet per second; ${ }^{\circ} \mathrm{C}$, degrees Celsius; $\mu \mathrm{g} / \mathrm{L}$, micrograms per liter; $\mu \mathrm{S} / \mathrm{cm}$, microsiemens per centimeter at $25^{\circ} \mathrm{C} ; \mathrm{mg} / \mathrm{L}$, milligrams per liter; $\mathrm{mm}$, millimeter; ton/d, tons per day. Symbols: $<$, less than laboratory reporting level $\left.{ }^{1}\right]$

\begin{tabular}{|c|c|c|c|c|c|}
\hline Property or constituent and reporting unit & $\begin{array}{l}\text { Number of } \\
\text { samples }\end{array}$ & Maximum & Minimum & Mean & Median \\
\hline \multicolumn{6}{|c|}{ 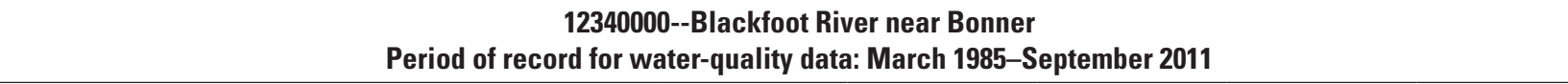 } \\
\hline Streamflow, instantaneous $\left(\mathrm{ft}^{3} / \mathrm{s}\right)$ & 184 & 13,400 & 344 & 2,790 & 1,340 \\
\hline pH, onsite (standard units) & 144 & 8.7 & 7.5 & 8.3 & 8.3 \\
\hline Specific conductance, onsite $(\mu \mathrm{S} / \mathrm{cm})$ & 161 & 294 & 131 & 208 & 204 \\
\hline Temperature, water $\left({ }^{\circ} \mathrm{C}\right)$ & 184 & 22.5 & 0.0 & 9.5 & 9.5 \\
\hline Hardness, filtered $\left(\mathrm{mg} / \mathrm{L}\right.$ as $\left.\mathrm{CaCO}_{3}\right)$ & 136 & 146 & 55.1 & 104 & 98.4 \\
\hline Calcium, filtered (mg/L) & 136 & 37.7 & 14.0 & 26.4 & 25.2 \\
\hline Magnesium, filtered (mg/L) & 136 & 13.2 & 4.90 & 9.12 & 8.66 \\
\hline Arsenic, filtered $(\mu \mathrm{g} / \mathrm{L})$ & 143 & 2.0 & $<1.0$ & ${ }^{2} .97$ & .96 \\
\hline Arsenic, unfiltered recoverable $(\mu \mathrm{g} / \mathrm{L})$ & 144 & 4.0 & $<1.0$ & ${ }^{2} 1.3$ & 1.0 \\
\hline Cadmium, filtered $(\mu \mathrm{g} / \mathrm{L})$ & 142 & 1.00 & $<.02$ & ${ }^{2} .02$ & $<.10$ \\
\hline Cadmium, unfiltered recoverable $(\mu \mathrm{g} / \mathrm{L})$ & 144 & 2.00 & $<.01$ & ${ }^{2} .09$ & $<1.00$ \\
\hline Copper, filtered $(\mu \mathrm{g} / \mathrm{L})$ & 140 & 7.0 & $<1.0$ & ${ }^{2} 1.3$ & .8 \\
\hline Copper, unfiltered recoverable ( $\mu \mathrm{g} / \mathrm{L})$ & 141 & 34.0 & $<1.0$ & ${ }^{2} 4.6$ & 2.0 \\
\hline Iron, filtered $(\mu \mathrm{g} / \mathrm{L})$ & 143 & 100 & $<3$ & ${ }^{2} 17$ & 10 \\
\hline Iron, unfiltered recoverable $(\mu \mathrm{g} / \mathrm{L})$ & 144 & 3,600 & 14 & 420 & 195 \\
\hline Lead, filtered $(\mu \mathrm{g} / \mathrm{L})$ & 138 & 8.00 & $<.01$ & ${ }^{2} .33$ & $<.60$ \\
\hline Lead, unfiltered recoverable $(\mu \mathrm{g} / \mathrm{L})$ & 140 & 25.0 & $<.60$ & ${ }^{2} 2.21$ & .08 \\
\hline Manganese, filtered $(\mu \mathrm{g} / \mathrm{L})$ & 143 & 11.0 & $<1.0$ & 22.4 & 2.0 \\
\hline Manganese, unfiltered recoverable $(\mu \mathrm{g} / \mathrm{L})$ & 144 & 180 & $<10.0$ & 228.9 & 18.6 \\
\hline Zinc, filtered $(\mu \mathrm{g} / \mathrm{L})$ & 142 & 15.0 & $<.60$ & ${ }^{2} 2.0$ & $<10.0$ \\
\hline Zinc, unfiltered recoverable $(\mu \mathrm{g} / \mathrm{L})$ & 144 & 60.0 & $<1.0$ & ${ }^{2} 5.3$ & $<10.0$ \\
\hline Sediment, suspended (percent finer than $0.062 \mathrm{~mm}$ ) & 182 & 98 & 42 & 80 & 82 \\
\hline Sediment, suspended concentration (mg/L) & 184 & 271 & 1 & 29 & 8 \\
\hline Sediment, suspended discharge (ton/d) & 184 & 7,670 & 1.1 & 533 & 31 \\
\hline
\end{tabular}


Table 25. Statistical summary of long-term water-quality data for the Clark Fork basin, Montana, March 1985 through September 2011.-Continued

[Abbreviations: $\mathrm{CaCO}_{3}$, calcium carbonate; $\mathrm{ft} / \mathrm{s}$, cubic feet per second; ${ }^{\circ} \mathrm{C}$, degrees Celsius; $\mu \mathrm{g} / \mathrm{L}$, micrograms per liter; $\mu \mathrm{S} / \mathrm{cm}$, microsiemens per centimeter at $25^{\circ} \mathrm{C} ; \mathrm{mg} / \mathrm{L}$, milligrams per liter; $\mathrm{mm}$, millimeter; ton/d, tons per day. Symbols: $<$, less than laboratory reporting level $\left.{ }^{1}\right]$

\begin{tabular}{|c|c|c|c|c|c|}
\hline Property or constituent and reporting unit & $\begin{array}{c}\text { Number of } \\
\text { samples }\end{array}$ & Maximum & Minimum & Mean & Median \\
\hline \multicolumn{6}{|c|}{$\begin{array}{l}\text { 12340500--Clark Fork above Missoula } \\
\text { eriod of record for water-quality data: July 1986-September } 2011\end{array}$} \\
\hline Streamflow, instantaneous $\left(\mathrm{ft}^{3} / \mathrm{s}\right)$ & 218 & 22,900 & 720 & 4,630 & 2,430 \\
\hline pH, onsite (standard units) & 175 & 8.8 & 7.9 & 8.3 & 8.3 \\
\hline Specific conductance, onsite $(\mu \mathrm{S} / \mathrm{cm})$ & 195 & 399 & 142 & 253 & 261 \\
\hline Temperature, water $\left({ }^{\circ} \mathrm{C}\right)$ & 215 & 22.0 & 0.0 & 9.6 & 9.5 \\
\hline Hardness, filtered $\left(\mathrm{mg} / \mathrm{L}\right.$ as $\left.\mathrm{CaCO}_{3}\right)$ & 175 & 168 & 60.5 & 116 & 118 \\
\hline Calcium, filtered (mg/L) & 175 & 46.0 & 14.0 & 31.4 & 31.7 \\
\hline Magnesium, filtered (mg/L) & 175 & 13.4 & 5.28 & 9.19 & 9.20 \\
\hline Arsenic, filtered $(\mu \mathrm{g} / \mathrm{L})$ & 175 & 9.0 & 1.0 & 3.5 & 3.2 \\
\hline Arsenic, unfiltered recoverable $(\mu \mathrm{g} / \mathrm{L})$ & 175 & 69.0 & 1.0 & 5.5 & 4.0 \\
\hline Cadmium, filtered $(\mu \mathrm{g} / \mathrm{L})$ & 174 & .20 & $<.04$ & ${ }^{2} .03$ & $<.10$ \\
\hline Cadmium, unfiltered recoverable $(\mu \mathrm{g} / \mathrm{L})$ & 175 & 5.0 & $<.01$ & ${ }^{2} .16$ & $<1.0$ \\
\hline Copper, filtered $(\mu \mathrm{g} / \mathrm{L})$ & 174 & 12.6 & .7 & 2.9 & 2.2 \\
\hline Copper, unfiltered recoverable $(\mu \mathrm{g} / \mathrm{L})$ & 173 & 400 & 2.0 & 20.1 & 8.8 \\
\hline Iron, filtered $(\mu \mathrm{g} / \mathrm{L})$ & 175 & 200 & $<3$ & 222 & 15 \\
\hline Iron, unfiltered recoverable $(\mu \mathrm{g} / \mathrm{L})$ & 175 & 13,000 & 43 & 647 & 260 \\
\hline Lead, filtered $(\mu \mathrm{g} / \mathrm{L})$ & 168 & 1.20 & $<.08$ & ${ }^{2} .15$ & $<1.00$ \\
\hline Lead, unfiltered recoverable $(\mu \mathrm{g} / \mathrm{L})$ & 170 & 78.0 & $<1.00$ & ${ }^{2} 3.57$ & 1.64 \\
\hline Manganese, filtered $(\mu \mathrm{g} / \mathrm{L})$ & 175 & 230 & 5.9 & 16.2 & 13.8 \\
\hline Manganese, unfiltered recoverable $(\mu \mathrm{g} / \mathrm{L})$ & 175 & 1,100 & 10.0 & 66.9 & 40.0 \\
\hline Zinc, filtered $(\mu \mathrm{g} / \mathrm{L})$ & 174 & 16.0 & $<1.0$ & ${ }^{2} 3.6$ & 2.4 \\
\hline Zinc, unfiltered recoverable $(\mu \mathrm{g} / \mathrm{L})$ & 175 & 1,100 & $<10.0$ & 234.4 & 15 \\
\hline Sediment, suspended (percent finer than $0.062 \mathrm{~mm}$ ) & 213 & 99 & 14 & 83 & 88 \\
\hline Sediment, suspended concentration $(\mathrm{mg} / \mathrm{L})$ & 218 & 950 & 2 & 45 & 12 \\
\hline Sediment, suspended discharge (ton/d) & 218 & 21,900 & 5.8 & 1,140 & 91 \\
\hline
\end{tabular}

${ }^{1}$ Differing less-than $(<)$ values for an individual constituent are the result of changes in the laboratory reporting level during the period of record.

${ }^{2}$ Value for the mean is estimated by using a log-probability regression to predict the values of data less than the laboratory reporting level (Helsel and Cohn, 1988). Minimum values that are not censored when the mean indicates that a censored value was used in the mean calculation, are a result of changes in the laboratory reporting level during the period of record. 
Table 26. Statistical summary of long-term bed-sediment data for the Clark Fork basin, Montana, August 1986 through August 2011.

[Reported concentrations are in micrograms per gram dry weight $(\mu \mathrm{g} / \mathrm{g})$. Number of samples represents the number of years that the constituent was analyzed, with each year represented by a single mean concentration of composite samples. Arsenic was not analyzed until 2003; therefore, the number of samples is smaller than that for the other trace elements. Values are reported using U.S. Geological Survey rounding standards. Symbols: <, less than the minimum reporting level; --, indicates insufficient data (less than three samples) to compute statistic]

\begin{tabular}{|c|c|c|c|c|c|}
\hline Constituent & Number of samples & Maximum & Minimum & Mean & Median \\
\hline \multicolumn{6}{|c|}{$\begin{array}{l}\text { 12323600--Silver Bow Creek at Opportunity } \\
\text { Period of record for bed-sediment data: 1992-2011 }\end{array}$} \\
\hline Cadmium & 20 & 43.9 & 6.8 & 28.6 & 28.2 \\
\hline Chromium & 18 & 50.7 & 16.8 & 29.5 & 26.7 \\
\hline Lead & 20 & 1,030 & 181 & 616 & 572 \\
\hline Manganese & 20 & 9,220 & 1,160 & 3,270 & 2,680 \\
\hline Nickel & 19 & 21.4 & 12.0 & 14.8 & 14.5 \\
\hline Silver & 12 & 20.0 & 8.3 & 15.5 & 15.8 \\
\hline
\end{tabular}

12323750--Silver Bow Creek at Warm Springs Period of record for bed-sediment data: 1992-2011

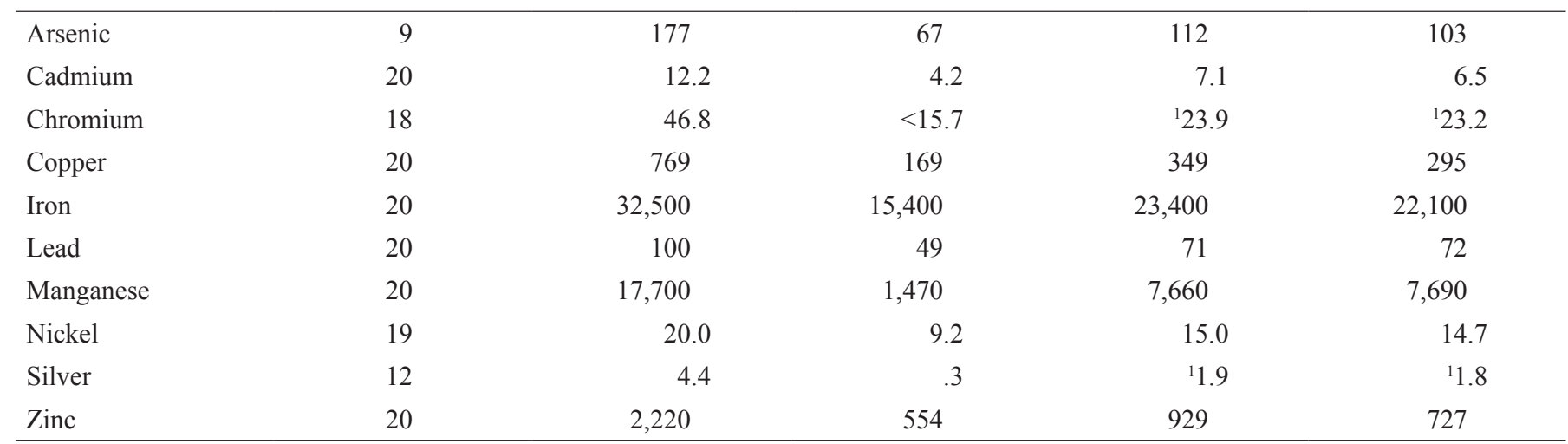

12323770--Warm Springs Creek at Warm Springs

Period of record for bed-sediment data: 1995, 1997, 1999, 2002, 2005, 2008, 2011

Arsenic
Cadmium
Chromium
Copper
Iron
Lead
Manganese
Nickel
Silver
Zinc

$\begin{array}{cccc}66 & 34 & 51 & 52 \\ 5.8 & 1.2 & 3.2 & 3.3 \\ 39.3 & 27.5 & 32.1 & 31.5 \\ 1,060 & 496 & 849 & 881 \\ 26,600 & 16,800 & 21,400 & 21,900 \\ 86 & 42 & 75 & 82 \\ 12,100 & 555 & 6,890 & 7,780 \\ 25.5 & 14.5 & 19.4 & 19.2 \\ 5.1 & 3.1 & 3.8 & 3.5 \\ 453 & 237 & 381 & 396\end{array}$


Table 26. Statistical summary of long-term bed-sediment data for the Clark Fork basin, Montana, August 1986 through August 2011.-Continued

[Reported concentrations are in micrograms per gram dry weight $(\mu \mathrm{g} / \mathrm{g})$. Number of samples represents the number of years that the constituent was analyzed, with each year represented by a single mean concentration of composite samples. Arsenic was not analyzed until 2003; therefore, the number of samples is smaller than that for the other trace elements. Values are reported using U.S. Geological Survey rounding standards. Symbols: $<$, less than the minimum reporting level; --, indicates insufficient data (less than three samples) to compute statistic]

\begin{tabular}{|c|c|c|c|c|c|}
\hline Constituent & Number of samples & Maximum & Minimum & Mean & Median \\
\hline \multicolumn{6}{|c|}{$\begin{array}{l}\text { 12323800--Clark Fork near Galen } \\
\text { Period of record for bed-sediment data: 1987, 1991-2011 }\end{array}$} \\
\hline Arsenic & 9 & 119 & 73 & 99 & 95 \\
\hline Cadmium & 22 & 20.1 & 3.8 & 8.2 & 7.3 \\
\hline Chromium & 18 & 44.6 & 19.1 & 29.4 & 28.3 \\
\hline Copper & 22 & 2,300 & 838 & 1,190 & 1,110 \\
\hline Iron & 22 & 39,800 & 22,600 & 27,700 & 26,900 \\
\hline Lead & 22 & 235 & 92 & 132 & 128 \\
\hline Manganese & 22 & 17,300 & 1,530 & 9,160 & 9,780 \\
\hline Nickel & 19 & 23.2 & 13.9 & 18.6 & 18.3 \\
\hline Silver & 14 & 7.3 & $<3.2$ & ${ }^{1} 4.4$ & ${ }^{1} 4.5$ \\
\hline Zinc & 22 & 3,560 & 721 & 1,420 & 1,140 \\
\hline
\end{tabular}

461415112450801--Clark Fork below Lost Creek, near Galen Period of record for bed-sediment data: 1996-2011

\begin{tabular}{|c|c|c|c|c|c|}
\hline Arsenic & 9 & 204 & 92 & 119 & 109 \\
\hline Cadmium & 16 & 10.0 & 4.8 & 6.9 & 6.7 \\
\hline Chromium & 15 & 42.4 & 20.5 & 30.4 & 31.8 \\
\hline Copper & 16 & 2,050 & 1,150 & 1,500 & 1,440 \\
\hline Iron & 16 & 32,800 & 24,400 & 29,300 & 29,900 \\
\hline Lead & 16 & 218 & 127 & 165 & 168 \\
\hline Manganese & 16 & 9,820 & 1,430 & 5,470 & 5,660 \\
\hline Nickel & 16 & 19.9 & 11.7 & 16.1 & 16.4 \\
\hline Silver & 8 & 7.8 & 4.2 & 6.5 & 6.7 \\
\hline Zinc & 16 & 1,680 & 930 & 1,300 & 1,290 \\
\hline
\end{tabular}

461559112443301--Clark Fork at county bridge, near Racetrack Period of record for bed-sediment data: 1996-2011

\begin{tabular}{|c|c|c|c|c|c|}
\hline Arsenic & 9 & 132 & 56 & 91 & 90 \\
\hline Cadmium & 16 & 8.7 & 5.0 & 6.6 & 6.4 \\
\hline Chromium & 15 & 45.2 & 19.0 & 29.2 & 29.2 \\
\hline Copper & 16 & 1,810 & 933 & 1,290 & 1,320 \\
\hline Iron & 16 & 31,700 & 21,200 & 27,500 & 28,400 \\
\hline Lead & 16 & 186 & 103 & 145 & 143 \\
\hline Manganese & 16 & 6,310 & 1,600 & 3,280 & 3,020 \\
\hline Nickel & 16 & 18.4 & 10.3 & 14.5 & 15.2 \\
\hline Silver & 8 & 6.1 & $<3.3$ & ${ }^{1} 5.0$ & ${ }^{1} 5.4$ \\
\hline Zinc & 16 & 1,550 & 999 & 1,200 & 1,180 \\
\hline
\end{tabular}


Table 26. Statistical summary of long-term bed-sediment data for the Clark Fork basin, Montana, August 1986 through August 2011.-Continued

[Reported concentrations are in micrograms per gram dry weight $(\mu \mathrm{g} / \mathrm{g})$. Number of samples represents the number of years that the constituent was analyzed, with each year represented by a single mean concentration of composite samples. Arsenic was not analyzed until 2003; therefore, the number of samples is smaller than that for the other trace elements. Values are reported using U.S. Geological Survey rounding standards. Symbols: <, less than the minimum reporting level; --, indicates insufficient data (less than three samples) to compute statistic]

\begin{tabular}{|c|c|c|c|c|c|}
\hline Constituent & Number of samples & Maximum & Minimum & Mean & Median \\
\hline \multicolumn{6}{|c|}{$\begin{array}{l}\text { 461903112440701--Clark Fork at Dempsey Creek diversion, near Racetrack } \\
\text { Period of record for bed-sediment data: 1996-2011 }\end{array}$} \\
\hline Cadmium & 16 & 10.3 & 4.1 & 6.2 & 5.8 \\
\hline Chromium & 15 & 39.2 & 16.0 & 27.7 & 26.4 \\
\hline Lead & 16 & 155 & 92 & 131 & 134 \\
\hline Manganese & 16 & 8,370 & 1,200 & 3,850 & 3,210 \\
\hline Nickel & 16 & 16.9 & 8.7 & 13.0 & 12.7 \\
\hline Silver & 8 & 6.2 & 2.7 & 4.9 & 5.0 \\
\hline
\end{tabular}

12324200--Clark Fork at Deer Lodge

Period of record for bed-sediment data: 1986-87, 1990-2011

\begin{tabular}{|c|c|c|c|c|c|}
\hline Arsenic & 9 & 102 & 49 & 73 & 70 \\
\hline Cadmium & 24 & 10.0 & 3.5 & 6.0 & 5.3 \\
\hline Chromium & 18 & 50.7 & 19.5 & 32.3 & 30.4 \\
\hline Iron & 24 & 35,300 & 21,100 & 27,400 & 26,300 \\
\hline Lead & 24 & 242 & 103 & 145 & 142 \\
\hline Silver & 16 & 7.9 & 2.4 & 4.7 & 4.5 \\
\hline Zinc & 24 & 1,730 & 844 & 1,190 & 1,140 \\
\hline
\end{tabular}

12324400--Clark Fork above Little Blackfoot River, near Garrison Period of record for bed-sediment data: 2009-2011

\begin{tabular}{|c|c|c|c|c|c|}
\hline Arsenic & 3 & 91 & 81 & 85 & 83 \\
\hline Cadmium & 3 & 5.5 & 4.2 & 4.8 & 4.8 \\
\hline Chromium & 3 & 52.8 & 45.5 & 48.3 & 46.4 \\
\hline Iron & 3 & 32,400 & 27,300 & 29,600 & 29,100 \\
\hline Lead & 3 & 145 & 140 & 143 & 144 \\
\hline Silver & 0 & -- & -- & -- & -- \\
\hline Zinc & 3 & 1,240 & 1,100 & 1,150 & 1,100 \\
\hline
\end{tabular}


Table 26. Statistical summary of long-term bed-sediment data for the Clark Fork basin, Montana, August 1986 through August 2011.-Continued

[Reported concentrations are in micrograms per gram dry weight $(\mu \mathrm{g} / \mathrm{g})$. Number of samples represents the number of years that the constituent was analyzed, with each year represented by a single mean concentration of composite samples. Arsenic was not analyzed until 2003; therefore, the number of samples is smaller than that for the other trace elements. Values are reported using U.S. Geological Survey rounding standards. Symbols: $<$, less than the minimum reporting level; --, indicates insufficient data (less than three samples) to compute statistic]

\begin{tabular}{|c|c|c|c|c|c|}
\hline Constituent & Number of samples & Maximum & Minimum & Mean & Median \\
\hline \multicolumn{6}{|c|}{$\begin{array}{l}\text { 12324680--Clark Fork at Goldcreek } \\
\text { Period of record for bed-sediment data: 1992-2011 }\end{array}$} \\
\hline Arsenic & 9 & 62 & 23 & 43 & 39 \\
\hline Cadmium & 20 & 8.1 & 2.6 & 4.6 & 4.1 \\
\hline Chromium & 18 & 55.3 & 21.3 & 34.1 & 32.6 \\
\hline Copper & 20 & 1,080 & 338 & 698 & 738 \\
\hline Iron & 20 & 32,100 & 15,500 & 24,000 & 24,400 \\
\hline Lead & 20 & 152 & 52 & 97 & 99 \\
\hline Manganese & 20 & 2,610 & 977 & 1,800 & 1,810 \\
\hline Nickel & 19 & 18.6 & 9.0 & 14.3 & 14.4 \\
\hline Silver & 12 & 4.8 & 2.3 & 3.3 & 3.2 \\
\hline Zinc & 20 & 1,320 & 584 & 929 & 944 \\
\hline
\end{tabular}

12331800--Clark Fork near Drummond

Period of record for bed-sediment data: 1986-87, 1991-2011

\begin{tabular}{|c|c|c|c|c|c|}
\hline Arsenic & 9 & 66 & 31 & 41 & 34 \\
\hline Cadmium & 23 & 7.7 & 1.7 & 4.3 & 4.3 \\
\hline Chromium & 18 & 41.9 & 17.0 & 29.8 & 31.4 \\
\hline Copper & 23 & 747 & 303 & 481 & 469 \\
\hline Iron & 23 & 43,700 & 16,500 & 24,900 & 23,200 \\
\hline Lead & 23 & 135 & 59 & 88 & 85 \\
\hline Manganese & 23 & 4,820 & 832 & 2,080 & 1,890 \\
\hline Nickel & 19 & 16.8 & 9.3 & 13.4 & 13.7 \\
\hline Silver & 15 & 4.7 & $<3.2$ & ${ }^{1} 3.0$ & ${ }^{1} 2.9$ \\
\hline Zinc & 23 & 1,230 & 637 & 946 & 948 \\
\hline
\end{tabular}

12334550--Clark Fork at Turah Bridge, near Bonner Period of record for bed-sediment data: 1986, 1991-2011

\begin{tabular}{|c|c|c|c|c|c|}
\hline Arsenic & 9 & 43 & 19 & 29 & 30 \\
\hline Cadmium & 22 & 7.3 & 1.9 & 3.5 & 3.5 \\
\hline Chromium & 18 & 42.5 & 15.3 & 27.2 & 28.4 \\
\hline Copper & 22 & 635 & 211 & 357 & 334 \\
\hline Iron & 22 & 25,900 & 12,600 & 19,500 & 17,400 \\
\hline Lead & 22 & 115 & 47 & 68 & 66 \\
\hline Manganese & 22 & 2,340 & 671 & 1,310 & 1,250 \\
\hline Nickel & 19 & 19.1 & 6.9 & 12.6 & 11.6 \\
\hline Silver & 14 & 3.9 & $<1.9$ & ${ }^{1} 2.1$ & ${ }^{1} 1.9$ \\
\hline Zinc & 22 & 1,160 & 584 & 808 & 786 \\
\hline
\end{tabular}


Table 26. Statistical summary of long-term bed-sediment data for the Clark Fork basin, Montana, August 1986 through August 2011.-Continued

[Reported concentrations are in micrograms per gram dry weight $(\mu \mathrm{g} / \mathrm{g})$. Number of samples represents the number of years that the constituent was analyzed, with each year represented by a single mean concentration of composite samples. Arsenic was not analyzed until 2003; therefore, the number of samples is smaller than that for the other trace elements. Values are reported using U.S. Geological Survey rounding standards. Symbols: <, less than the minimum reporting level; --, indicates insufficient data (less than three samples) to compute statistic]

\begin{tabular}{|c|c|c|c|c|c|}
\hline Constituent & Number of samples & Maximum & Minimum & Mean & Median \\
\hline \multicolumn{6}{|c|}{ 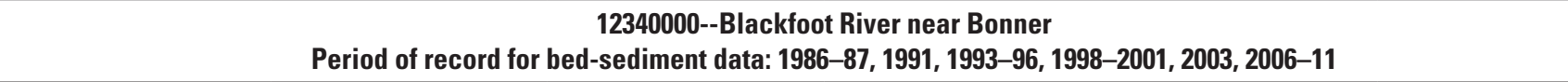 } \\
\hline Cadmium & 18 & 2.0 & .05 & ${ }^{1} .5$ & ${ }^{1} .3$ \\
\hline Chromium & 14 & 35.2 & 15.1 & 23.0 & 23.7 \\
\hline Lead & 18 & 20 & $<13$ & ${ }^{1} 13$ & ${ }^{1} 12$ \\
\hline Manganese & 18 & 746 & 298 & 530 & 543 \\
\hline Nickel & 15 & 14.3 & 6.0 & 11.3 & 11.7 \\
\hline Silver & 12 & $<1.9$ & $<.3$ & ${ }^{1} .5$ & ${ }^{1<}<.6$ \\
\hline
\end{tabular}

12340500--Clark Fork above Missoula

Period of record for bed-sediment data: 1997-2011

\begin{tabular}{|c|c|c|c|c|c|}
\hline Arsenic & 9 & 54 & 17 & 34 & 30 \\
\hline Cadmium & 15 & 5.8 & 1.2 & 3.1 & 2.8 \\
\hline Chromium & 14 & 40.7 & 19.0 & 27.7 & 29.0 \\
\hline Iron & 15 & 27,000 & 18,100 & 21,100 & 20,500 \\
\hline Lead & 15 & 78 & 37 & 56 & 57 \\
\hline Silver & 7 & 2.9 & .8 & ${ }^{1} 2.0$ & ${ }^{1} 2.1$ \\
\hline Zinc & 15 & 1,090 & 438 & 740 & 716 \\
\hline
\end{tabular}

${ }^{1}$ Value determined by substituting one-half of the minimum reporting level for censored $(<)$ values when both uncensored and censored values were used to determine the mean and (or) median. 
Table 27. Statistical summary of long-term biological data for the Clark Fork basin, Montana, August 1986 through August 2011.

[Concentrations are in micrograms per gram dry weight $(\mu \mathrm{g} / \mathrm{g})$. Number of composite samples represents the total of all individual composite samples collected for every year that the constituent was analyzed. Values for a single sample are arbitrarily listed in the "Mean" column. Because Hydropsyche insects were not sorted to the species level during 1986-89, Hydropsyche species statistics for stations sampled during those years are based on the results of all Hydropsyche species combined. At some sites, statistics of Hydropsyche morosa group are based on the combined results of two or more species. Insects collected during 1986-98 were depurated prior to analysis; depuration was discontinued in 1999. Arsenic was not analyzed until 2003; therefore, the number of samples may be small or zero for some taxa. Values are reported using U.S. Geological Survey rounding standards. Abbreviation: spp., one or more similar species. Symbols: $<$, less than minimum reporting level; --, indicates either too few samples (less than three) or insufficient data to compute statistic, or element not analyzed]

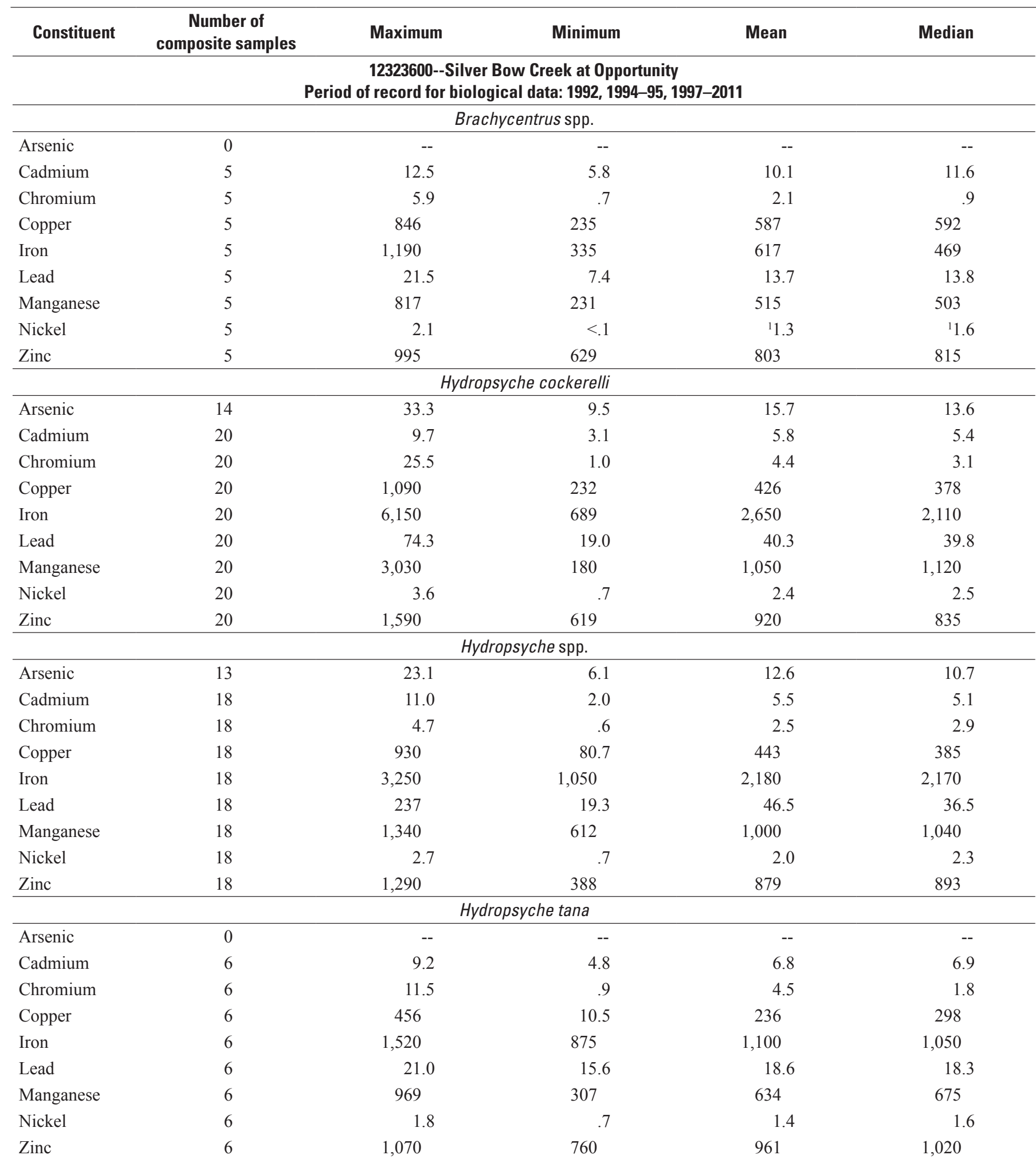


Table 27. Statistical summary of long-term biological data for the Clark Fork basin, Montana, August 1986 through August 2011.-Continued

[Concentrations are in micrograms per gram dry weight $(\mu \mathrm{g} / \mathrm{g})$. Number of composite samples represents the total of all individual composite samples collected for every year that the constituent was analyzed. Values for a single sample are arbitrarily listed in the "Mean" column. Because Hydropsyche insects were not sorted to the species level during 1986-89, Hydropsyche species statistics for stations sampled during those years are based on the results of all Hydropsyche species combined. At some sites, statistics of Hydropsyche morosa group are based on the combined results of two or more species. Insects collected during 1986-98 were depurated prior to analysis; depuration was discontinued in 1999. Arsenic was not analyzed until 2003; therefore, the number of samples may be small or zero for some taxa. Values are reported using U.S. Geological Survey rounding standards. Abbreviation: spp., one or more similar species. Symbols: $<$, less than minimum reporting level; --, indicates either too few samples (less than three) or insufficient data to compute statistic, or element not analyzed]

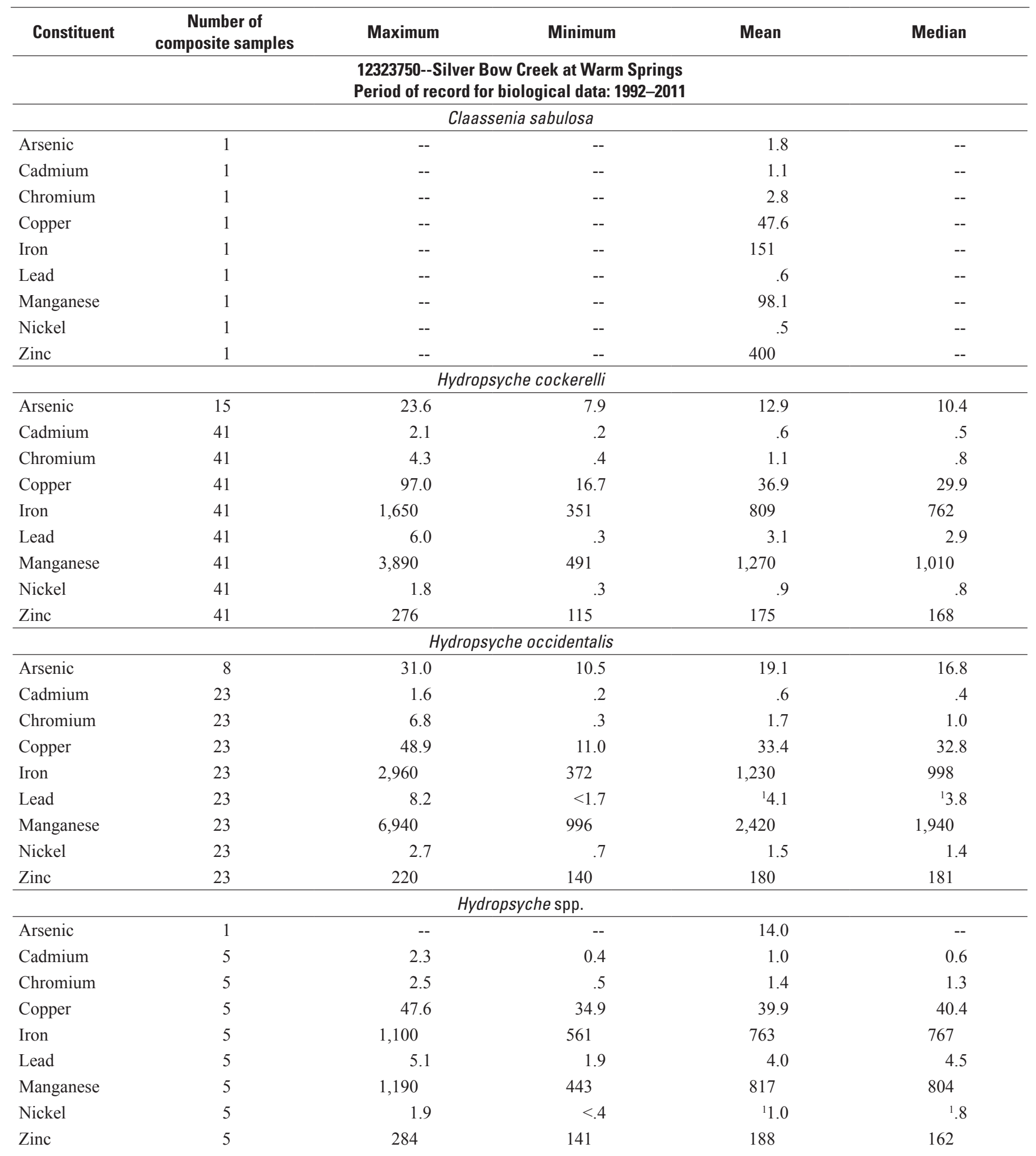


Table 27. Statistical summary of long-term biological data for the Clark Fork basin, Montana, August 1986 through August 2011.-Continued

[Concentrations are in micrograms per gram dry weight $(\mu \mathrm{g} / \mathrm{g})$. Number of composite samples represents the total of all individual composite samples collected for every year that the constituent was analyzed. Values for a single sample are arbitrarily listed in the "Mean" column. Because Hydropsyche insects were not sorted to the species level during 1986-89, Hydropsyche species statistics for stations sampled during those years are based on the results of all Hydropsyche species combined. At some sites, statistics of Hydropsyche morosa group are based on the combined results of two or more species. Insects collected during 1986-98 were depurated prior to analysis; depuration was discontinued in 1999. Arsenic was not analyzed until 2003; therefore, the number of samples may be small or zero for some taxa. Values are reported using U.S. Geological Survey rounding standards. Abbreviation: spp., one or more similar species. Symbols: $<$, less than minimum reporting level; --, indicates either too few samples (less than three) or insufficient data to compute statistic, or element not analyzed]

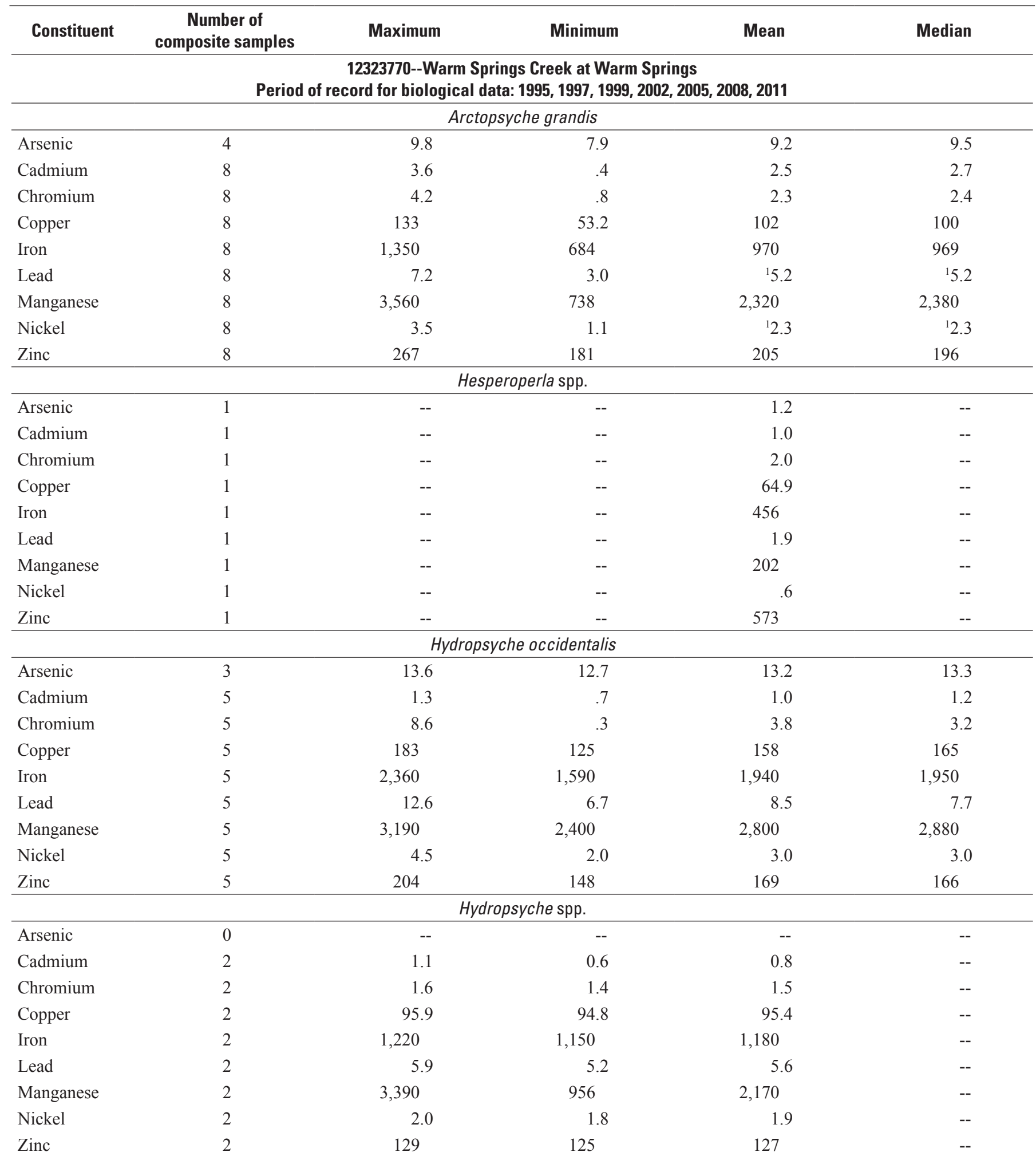


Table 27. Statistical summary of long-term biological data for the Clark Fork basin, Montana, August 1986 through August 2011.-Continued

[Concentrations are in micrograms per gram dry weight $(\mu \mathrm{g} / \mathrm{g})$. Number of composite samples represents the total of all individual composite samples collected for every year that the constituent was analyzed. Values for a single sample are arbitrarily listed in the "Mean" column. Because Hydropsyche insects were not sorted to the species level during 1986-89, Hydropsyche species statistics for stations sampled during those years are based on the results of all Hydropsyche species combined. At some sites, statistics of Hydropsyche morosa group are based on the combined results of two or more species. Insects collected during 1986-98 were depurated prior to analysis; depuration was discontinued in 1999. Arsenic was not analyzed until 2003; therefore, the number of samples may be small or zero for some taxa. Values are reported using U.S. Geological Survey rounding standards. Abbreviation: spp., one or more similar species. Symbols: $<$, less than minimum reporting level; --, indicates either too few samples (less than three) or insufficient data to compute statistic, or element not analyzed]

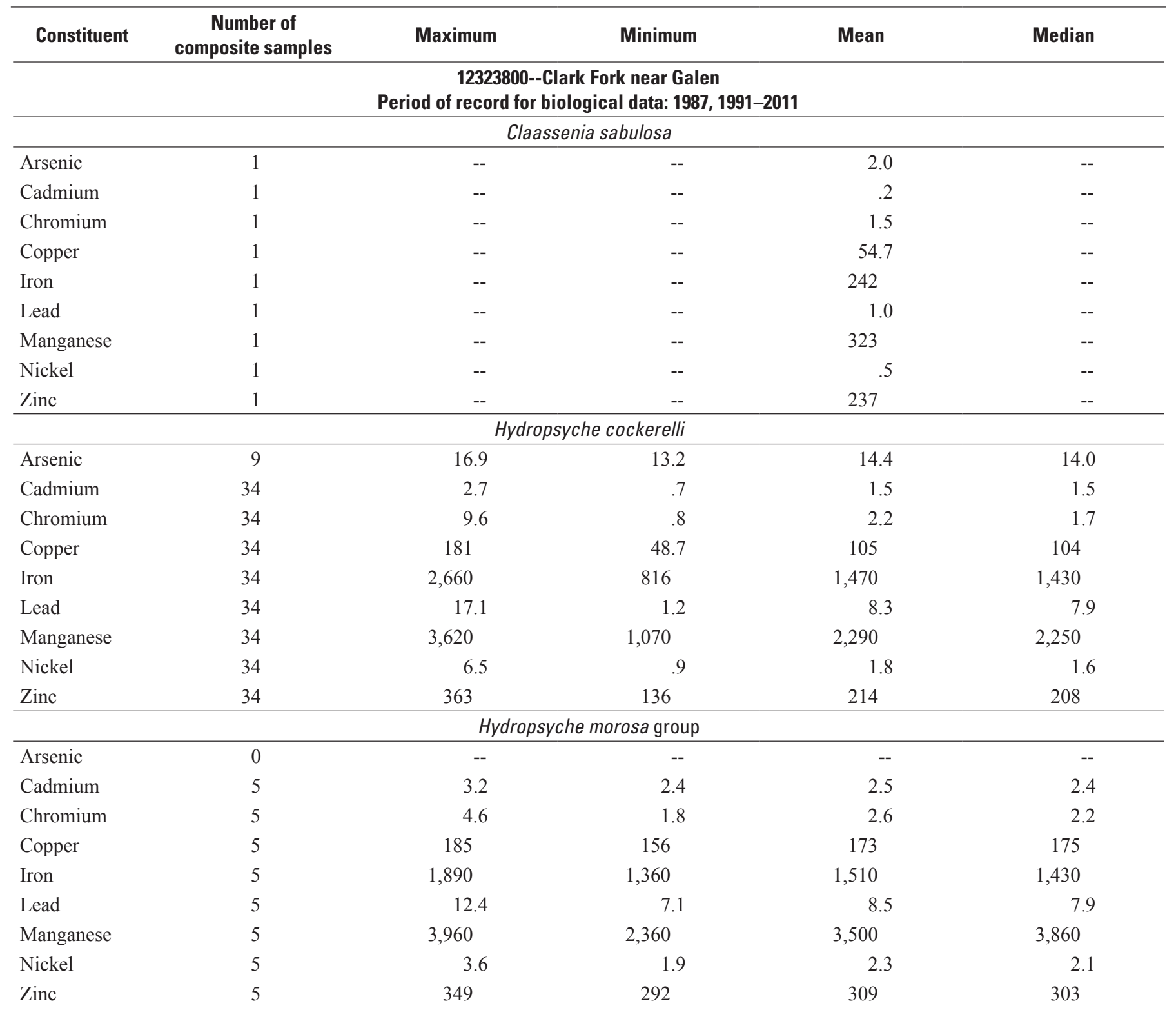


Table 27. Statistical summary of long-term biological data for the Clark Fork basin, Montana, August 1986 through August 2011.-Continued

[Concentrations are in micrograms per gram dry weight $(\mu \mathrm{g} / \mathrm{g})$. Number of composite samples represents the total of all individual composite samples collected for every year that the constituent was analyzed. Values for a single sample are arbitrarily listed in the "Mean" column. Because Hydropsyche insects were not sorted to the species level during 1986-89, Hydropsyche species statistics for stations sampled during those years are based on the results of all Hydropsyche species combined. At some sites, statistics of Hydropsyche morosa group are based on the combined results of two or more species. Insects collected during 1986-98 were depurated prior to analysis; depuration was discontinued in 1999. Arsenic was not analyzed until 2003; therefore, the number of samples may be small or zero for some taxa. Values are reported using U.S. Geological Survey rounding standards. Abbreviation: spp., one or more similar species. Symbols: $<$, less than minimum reporting level; --, indicates either too few samples (less than three) or insufficient data to compute statistic, or element not analyzed]

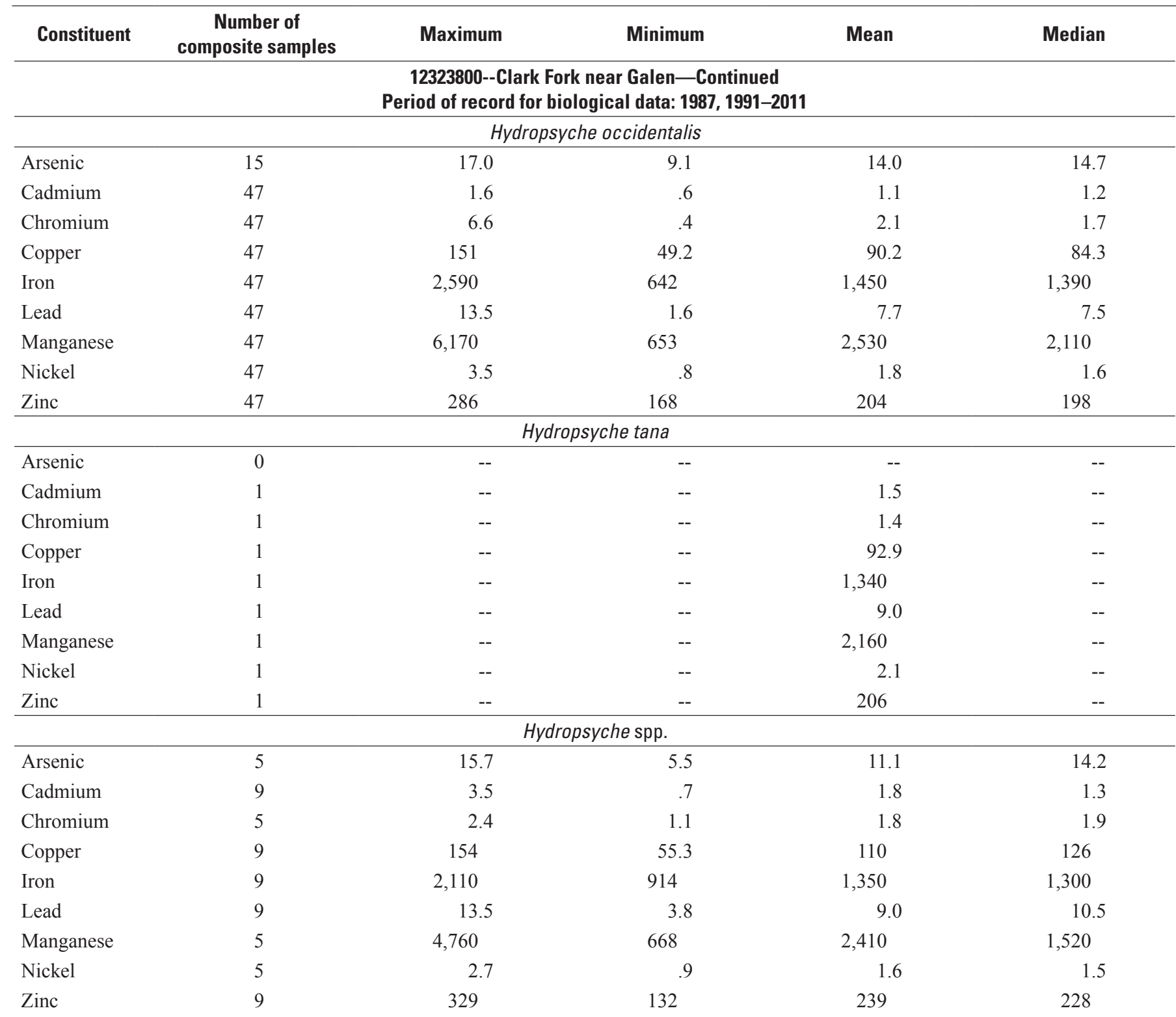


Table 27. Statistical summary of long-term biological data for the Clark Fork basin, Montana, August 1986 through August 2011.-Continued

[Concentrations are in micrograms per gram dry weight $(\mu \mathrm{g} / \mathrm{g})$. Number of composite samples represents the total of all individual composite samples collected for every year that the constituent was analyzed. Values for a single sample are arbitrarily listed in the "Mean" column. Because Hydropsyche insects were not sorted to the species level during 1986-89, Hydropsyche species statistics for stations sampled during those years are based on the results of all Hydropsyche species combined. At some sites, statistics of Hydropsyche morosa group are based on the combined results of two or more species. Insects collected during 1986-98 were depurated prior to analysis; depuration was discontinued in 1999. Arsenic was not analyzed until 2003; therefore, the number of samples may be small or zero for some taxa. Values are reported using U.S. Geological Survey rounding standards. Abbreviation: spp., one or more similar species. Symbols: $<$, less than minimum reporting level; --, indicates either too few samples (less than three) or insufficient data to compute statistic, or element not analyzed]

\begin{tabular}{|c|c|c|c|c|c|}
\hline Constituent & $\begin{array}{c}\text { Number of } \\
\text { composite samples }\end{array}$ & Maximum & Minimum & Mean & Median \\
\hline \multicolumn{6}{|c|}{$\begin{array}{l}\text { 461415112450801--Clark Fork below Lost Creek, near Galen } \\
\text { Period of record for biological data: 1996-2011 }\end{array}$} \\
\hline \multicolumn{6}{|c|}{ Claassenia sabulosa } \\
\hline Arsenic & 1 & -- & -- & 1.5 & -- \\
\hline Cadmium & 2 & 0.4 & 0.3 & .4 & -- \\
\hline Copper & 2 & 70.1 & 67.1 & 68.6 & -- \\
\hline Iron & 2 & 209 & 189 & 199 & -- \\
\hline Lead & 2 & 1.2 & .7 & 1.0 & -- \\
\hline Manganese & 2 & 238 & 90.4 & 164 & -- \\
\hline Nickel & 2 & .2 & $<.2$ & ${ }^{1} .2$ & -- \\
\hline Cadmium & 22 & 2.8 & 1.1 & 1.8 & 1.6 \\
\hline Chromium & 22 & 3.6 & .8 & 2.0 & 2.0 \\
\hline Copper & 22 & 338 & 48.8 & 134 & 113 \\
\hline Iron & 22 & 4,080 & 691 & 1,530 & 1,180 \\
\hline Lead & 22 & 28.6 & 4.5 & 11.6 & 9.0 \\
\hline Manganese & 22 & 3,160 & 1,230 & 1,850 & 1,720 \\
\hline Nickel & 22 & 2.8 & .9 & 1.4 & 1.2 \\
\hline Zinc & 22 & 339 & 151 & 228 & 223 \\
\hline \multicolumn{6}{|c|}{ Hydropsyche occidentalis } \\
\hline Arsenic & 9 & 20.9 & 12.7 & 15.8 & 15.0 \\
\hline Nickel & 23 & 3.0 & .9 & 1.6 & 1.5 \\
\hline Zinc & 23 & 308 & 174 & 243 & 245 \\
\hline
\end{tabular}


Table 27. Statistical summary of long-term biological data for the Clark Fork basin, Montana, August 1986 through August 2011.-Continued

[Concentrations are in micrograms per gram dry weight $(\mu \mathrm{g} / \mathrm{g})$. Number of composite samples represents the total of all individual composite samples collected for every year that the constituent was analyzed. Values for a single sample are arbitrarily listed in the "Mean" column. Because Hydropsyche insects were not sorted to the species level during 1986-89, Hydropsyche species statistics for stations sampled during those years are based on the results of all Hydropsyche species combined. At some sites, statistics of Hydropsyche morosa group are based on the combined results of two or more species. Insects collected during 1986-98 were depurated prior to analysis; depuration was discontinued in 1999. Arsenic was not analyzed until 2003; therefore, the number of samples may be small or zero for some taxa. Values are reported using U.S. Geological Survey rounding standards. Abbreviation: spp., one or more similar species. Symbols: $<$, less than minimum reporting level; --, indicates either too few samples (less than three) or insufficient data to compute statistic, or element not analyzed]

\begin{tabular}{|c|c|c|c|c|c|}
\hline Constituent & $\begin{array}{c}\text { Number of } \\
\text { composite samples }\end{array}$ & Maximum & Minimum & Mean & Median \\
\hline \multicolumn{6}{|c|}{$\begin{array}{l}\text { 461415112450801--Clark Fork below Lost Creek, near Galen-Continued } \\
\text { Period of record for biological data: 1996-2011 }\end{array}$} \\
\hline Arsenic & 4 & 14.5 & 7.0 & 10.2 & 9.7 \\
\hline Cadmium & 8 & 1.8 & 1.0 & 1.3 & 1.3 \\
\hline Copper & 8 & 153 & 45.1 & 96.4 & 93.0 \\
\hline Iron & 8 & 1,810 & 533 & 1,160 & 1,130 \\
\hline Lead & 8 & 20.5 & 4.1 & 9.5 & 8.0 \\
\hline Manganese & 8 & 1,980 & 775 & 1,270 & 1,230 \\
\hline Nickel & 8 & 2.8 & .9 & 1.6 & 1.4 \\
\hline Cadmium & 2 & 4.3 & 3.9 & 4.1 & -- \\
\hline Chromium & 2 & 1.1 & 1.0 & 1.0 & -- \\
\hline Copper & 2 & 93.1 & 73.7 & 83.4 & -- \\
\hline Iron & 2 & 346 & 324 & 335 & -- \\
\hline Lead & 2 & 5.9 & 4.8 & 5.4 & -- \\
\hline Manganese & 2 & 320 & 192 & 256 & -- \\
\hline Nickel & 2 & .3 & .3 & .3 & -- \\
\hline Zinc & 2 & 411 & 301 & 356 & -- \\
\hline
\end{tabular}


Table 27. Statistical summary of long-term biological data for the Clark Fork basin, Montana, August 1986 through August 2011.-Continued

[Concentrations are in micrograms per gram dry weight $(\mu \mathrm{g} / \mathrm{g})$. Number of composite samples represents the total of all individual composite samples collected for every year that the constituent was analyzed. Values for a single sample are arbitrarily listed in the "Mean" column. Because Hydropsyche insects were not sorted to the species level during 1986-89, Hydropsyche species statistics for stations sampled during those years are based on the results of all Hydropsyche species combined. At some sites, statistics of Hydropsyche morosa group are based on the combined results of two or more species. Insects collected during 1986-98 were depurated prior to analysis; depuration was discontinued in 1999. Arsenic was not analyzed until 2003; therefore, the number of samples may be small or zero for some taxa. Values are reported using U.S. Geological Survey rounding standards. Abbreviation: spp., one or more similar species. Symbols: $<$, less than minimum reporting level; --, indicates either too few samples (less than three) or insufficient data to compute statistic, or element not analyzed]

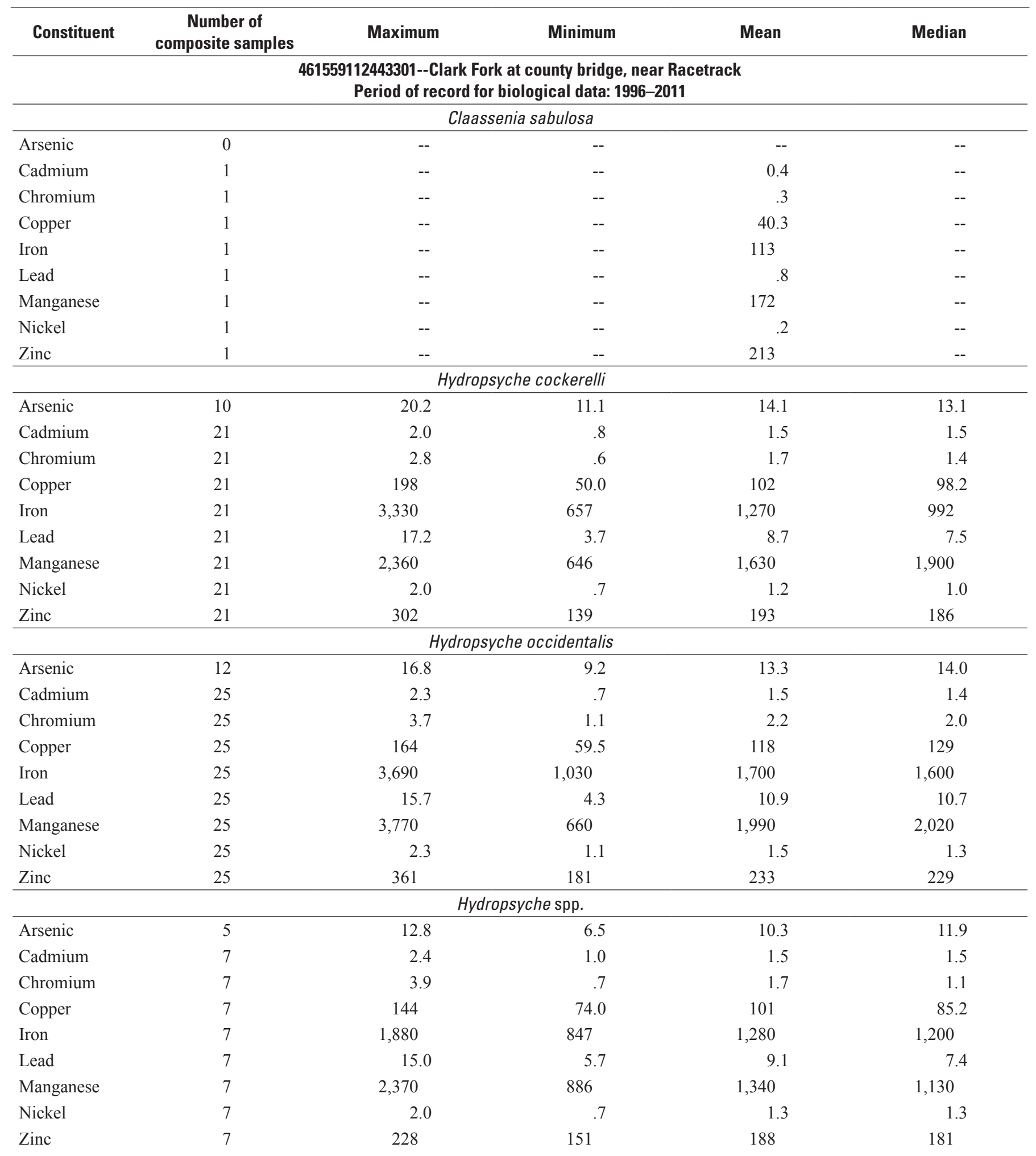


Table 27. Statistical summary of long-term biological data for the Clark Fork basin, Montana, August 1986 through August 2011.-Continued

[Concentrations are in micrograms per gram dry weight $(\mu \mathrm{g} / \mathrm{g})$. Number of composite samples represents the total of all individual composite samples collected for every year that the constituent was analyzed. Values for a single sample are arbitrarily listed in the "Mean" column. Because Hydropsyche insects were not sorted to the species level during 1986-89, Hydropsyche species statistics for stations sampled during those years are based on the results of all Hydropsyche species combined. At some sites, statistics of Hydropsyche morosa group are based on the combined results of two or more species. Insects collected during 1986-98 were depurated prior to analysis; depuration was discontinued in 1999. Arsenic was not analyzed until 2003; therefore, the number of samples may be small or zero for some taxa. Values are reported using U.S. Geological Survey rounding standards. Abbreviation: spp., one or more similar species. Symbols: $<$, less than minimum reporting level; --, indicates either too few samples (less than three) or insufficient data to compute statistic, or element not analyzed]

\begin{tabular}{|c|c|c|c|c|c|}
\hline Constituent & $\begin{array}{c}\text { Number of } \\
\text { composite samples }\end{array}$ & Maximum & Minimum & Mean & Median \\
\hline \multicolumn{6}{|c|}{$\begin{array}{c}\text { 461903112440701--Clark Fork at Dempsey Creek diversion, near Racetrack } \\
\text { Period of record for biological data: 1996-2011 }\end{array}$} \\
\hline \multicolumn{6}{|c|}{ Arctopsyche grandis } \\
\hline Arsenic & 1 & -- & -- & 11.8 & -- \\
\hline Cadmium & 2 & 7.1 & 1.7 & 4.4 & -- \\
\hline Copper & 2 & 151 & 30.8 & 90.9 & -- \\
\hline Iron & 2 & 1,500 & 340 & 920 & -- \\
\hline Lead & 2 & 12.4 & $<14.5$ & 19.8 & -- \\
\hline Manganese & 2 & 1,190 & 510 & 850 & -- \\
\hline Nickel & 2 & 2.3 & 1.0 & 1.6 & -- \\
\hline \multicolumn{6}{|c|}{ Claassenia sabulosa } \\
\hline Cadmium & 1 & -- & -- & 2.4 & -- \\
\hline Chromium & 1 & -- & -- & 1.7 & -- \\
\hline Copper & 1 & -- & -- & 73.4 & -- \\
\hline Iron & 1 & -- & -- & 297 & -- \\
\hline Lead & 1 & -- & -- & 1.9 & -- \\
\hline Manganese & 1 & -- & -- & 115 & -- \\
\hline Nickel & 1 & -- & -- & .4 & -- \\
\hline Zinc & 1 & -- & -- & 330 & -- \\
\hline \multicolumn{6}{|c|}{ Hydropsyche cockerelli } \\
\hline Arsenic & 9 & 18.8 & 8.0 & 13.0 & 10.4 \\
\hline Nickel & 18 & 2.5 & .5 & 1.2 & 1.0 \\
\hline Zinc & 18 & 279 & 162 & 208 & 190 \\
\hline
\end{tabular}


Table 27. Statistical summary of long-term biological data for the Clark Fork basin, Montana, August 1986 through August 2011.-Continued

[Concentrations are in micrograms per gram dry weight $(\mu \mathrm{g} / \mathrm{g})$. Number of composite samples represents the total of all individual composite samples collected for every year that the constituent was analyzed. Values for a single sample are arbitrarily listed in the "Mean" column. Because Hydropsyche insects were not sorted to the species level during 1986-89, Hydropsyche species statistics for stations sampled during those years are based on the results of all Hydropsyche species combined. At some sites, statistics of Hydropsyche morosa group are based on the combined results of two or more species. Insects collected during 1986-98 were depurated prior to analysis; depuration was discontinued in 1999. Arsenic was not analyzed until 2003; therefore, the number of samples may be small or zero for some taxa. Values are reported using U.S. Geological Survey rounding standards. Abbreviation: spp., one or more similar species. Symbols: $<$, less than minimum reporting level; --, indicates either too few samples (less than three) or insufficient data to compute statistic, or element not analyzed]

\begin{tabular}{|c|c|c|c|c|c|}
\hline Constituent & $\begin{array}{c}\text { Number of } \\
\text { composite samples }\end{array}$ & Maximum & Minimum & Mean & Median \\
\hline \multicolumn{6}{|c|}{$\begin{array}{l}\text { 461903112440701--Clark Fork at Dempsey Creek diversion, near Racetrack-Continued } \\
\text { Period of record for biological data: 1996-2011 }\end{array}$} \\
\hline \multicolumn{6}{|c|}{ Hydropsyche occidentalis } \\
\hline Arsenic & 15 & 24.0 & 10.2 & 15.6 & 15.9 \\
\hline Cadmium & 32 & 2.4 & .7 & 1.4 & 1.3 \\
\hline Copper & 32 & 345 & 74.9 & 130 & 112 \\
\hline Iron & 32 & 3,390 & 940 & 1,780 & 1,550 \\
\hline Lead & 32 & 21.8 & 6.1 & 12.9 & 11.8 \\
\hline Manganese & 32 & 4,460 & 826 & 2,410 & 2,290 \\
\hline Nickel & 32 & 2.4 & 1.1 & 1.6 & 1.5 \\
\hline Cadmium & 4 & 1.7 & .9 & 1.3 & 1.3 \\
\hline Chromium & 4 & 2.1 & .8 & 1.4 & 1.2 \\
\hline Copper & 4 & 140 & 65.5 & 94.1 & 85.4 \\
\hline Iron & 4 & 1,610 & 875 & 1,120 & 987 \\
\hline Lead & 4 & 13.2 & 7.3 & 9.7 & 9.1 \\
\hline Manganese & 4 & 1,150 & 638 & 824 & 756 \\
\hline Nickel & 4 & 1.6 & .6 & 1.1 & 1.1 \\
\hline Zinc & 4 & 212 & 162 & 184 & 180 \\
\hline
\end{tabular}


Table 27. Statistical summary of long-term biological data for the Clark Fork basin, Montana, August 1986 through August 2011.-Continued

[Concentrations are in micrograms per gram dry weight $(\mu \mathrm{g} / \mathrm{g})$. Number of composite samples represents the total of all individual composite samples collected for every year that the constituent was analyzed. Values for a single sample are arbitrarily listed in the "Mean" column. Because Hydropsyche insects were not sorted to the species level during 1986-89, Hydropsyche species statistics for stations sampled during those years are based on the results of all Hydropsyche species combined. At some sites, statistics of Hydropsyche morosa group are based on the combined results of two or more species. Insects collected during 1986-98 were depurated prior to analysis; depuration was discontinued in 1999. Arsenic was not analyzed until 2003; therefore, the number of samples may be small or zero for some taxa. Values are reported using U.S. Geological Survey rounding standards. Abbreviation: spp., one or more similar species. Symbols: $<$, less than minimum reporting level; --, indicates either too few samples (less than three) or insufficient data to compute statistic, or element not analyzed]

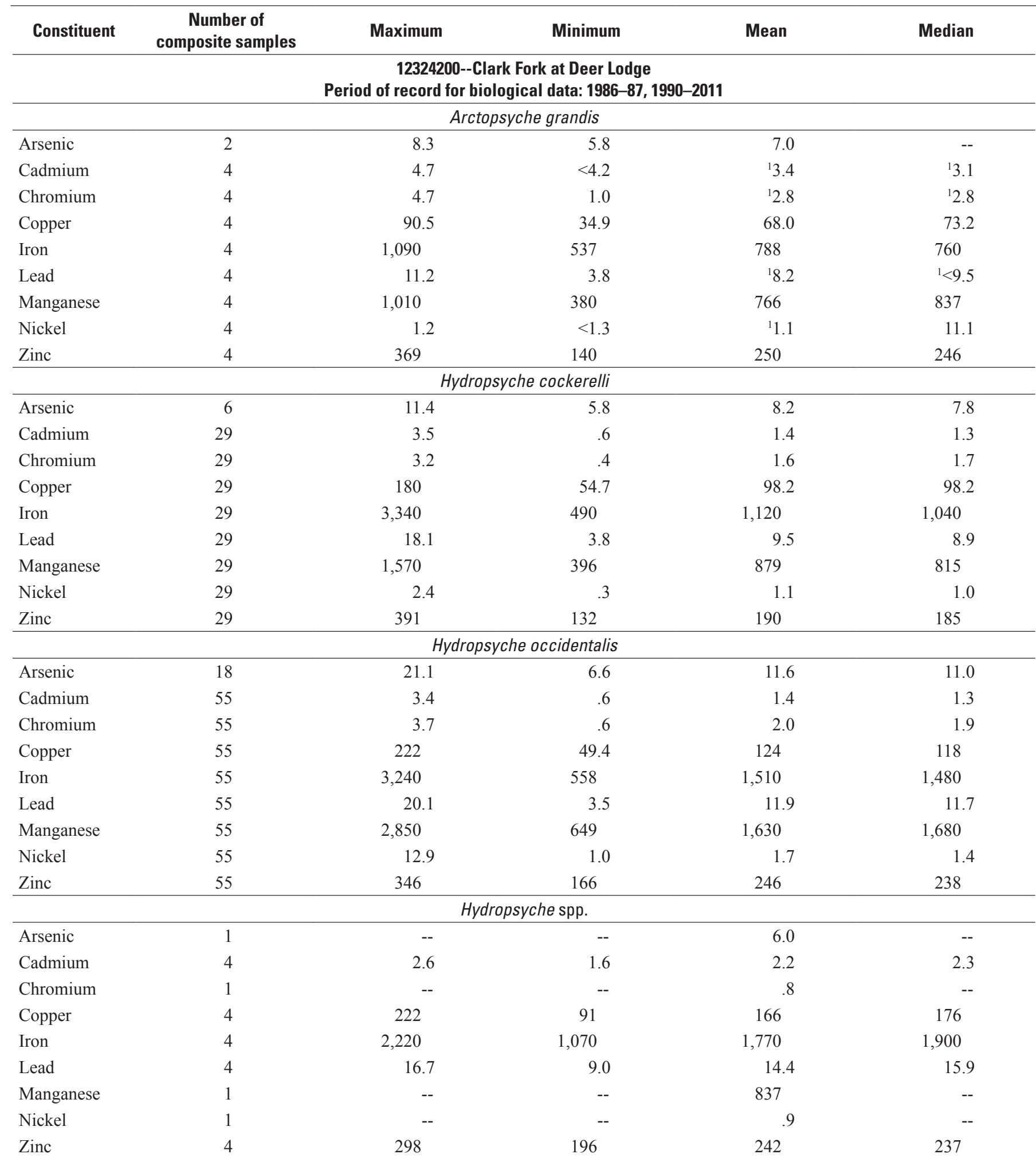


Table 27. Statistical summary of long-term biological data for the Clark Fork basin, Montana, August 1986 through August 2011.-Continued

[Concentrations are in micrograms per gram dry weight $(\mu \mathrm{g} / \mathrm{g})$. Number of composite samples represents the total of all individual composite samples collected for every year that the constituent was analyzed. Values for a single sample are arbitrarily listed in the "Mean" column. Because Hydropsyche insects were not sorted to the species level during 1986-89, Hydropsyche species statistics for stations sampled during those years are based on the results of all Hydropsyche species combined. At some sites, statistics of Hydropsyche morosa group are based on the combined results of two or more species. Insects collected during 1986-98 were depurated prior to analysis; depuration was discontinued in 1999. Arsenic was not analyzed until 2003; therefore, the number of samples may be small or zero for some taxa. Values are reported using U.S. Geological Survey rounding standards. Abbreviation: spp., one or more similar species. Symbols: $<$, less than minimum reporting level; --, indicates either too few samples (less than three) or insufficient data to compute statistic, or element not analyzed]

\begin{tabular}{|c|c|c|c|c|c|}
\hline Constituent & $\begin{array}{c}\text { Number of } \\
\text { composite samples }\end{array}$ & Maximum & Minimum & Mean & Median \\
\hline \multicolumn{6}{|c|}{$\begin{array}{l}\text { 12324400--Clark Fork above Little Blackfoot River, near Garrison } \\
\text { Period of record for biological data: 2009-11 }\end{array}$} \\
\hline \multicolumn{6}{|c|}{ Arctopsyche grandis } \\
\hline Arsenic & 3 & 16.6 & 5.5 & 9.4 & 6.0 \\
\hline Cadmium & 3 & 4.9 & 3.2 & 3.8 & 3.2 \\
\hline Copper & 3 & 209 & 65.7 & 120 & 83.6 \\
\hline Iron & 3 & 2,580 & 694 & 1,370 & 826 \\
\hline Lead & 3 & 18.0 & 6.6 & 10.9 & 8.1 \\
\hline Manganese & 3 & 990 & 940 & 967 & 972 \\
\hline Nickel & 3 & 2.2 & .7 & 1.2 & .8 \\
\hline \multicolumn{6}{|c|}{ Hydropsyche cockerelli } \\
\hline Cadmium & 1 & -- & -- & 4.0 & -- \\
\hline Chromium & 1 & -- & -- & 3.4 & -- \\
\hline Copper & 1 & -- & -- & 158 & -- \\
\hline Iron & 1 & -- & -- & 2,150 & -- \\
\hline Lead & 1 & -- & -- & 18.8 & -- \\
\hline Manganese & 1 & -- & -- & 1,500 & -- \\
\hline Nickel & 1 & -- & -- & 1.7 & -- \\
\hline Zinc & 1 & -- & -- & 284 & -- \\
\hline \multicolumn{6}{|c|}{ Hydropsyche occidentalis } \\
\hline Arsenic & 6 & 14.7 & 7.9 & 11.3 & 11.7 \\
\hline Nickel & 6 & 1.9 & 1.0 & 1.4 & 1.6 \\
\hline Zinc & 6 & 299 & 223 & 257 & 258 \\
\hline \multicolumn{6}{|c|}{ Hydropsyche spp. } \\
\hline Arsenic & 1 & -- & -- & 13.6 & -- \\
\hline Cadmium & 1 & -- & -- & 1.7 & -- \\
\hline Chromium & 1 & -- & -- & 4.3 & -- \\
\hline Copper & 1 & -- & -- & 187 & -- \\
\hline Iron & 1 & -- & -- & 2,570 & -- \\
\hline Lead & 1 & -- & -- & 18.5 & -- \\
\hline Manganese & 1 & -- & -- & 919 & -- \\
\hline Nickel & 1 & -- & -- & 1.8 & -- \\
\hline Zinc & 1 & -- & -- & 296 & -- \\
\hline
\end{tabular}


Table 27. Statistical summary of long-term biological data for the Clark Fork basin, Montana, August 1986 through August 2011.-Continued

[Concentrations are in micrograms per gram dry weight $(\mu \mathrm{g} / \mathrm{g})$. Number of composite samples represents the total of all individual composite samples collected for every year that the constituent was analyzed. Values for a single sample are arbitrarily listed in the "Mean" column. Because Hydropsyche insects were not sorted to the species level during 1986-89, Hydropsyche species statistics for stations sampled during those years are based on the results of all Hydropsyche species combined. At some sites, statistics of Hydropsyche morosa group are based on the combined results of two or more species. Insects collected during 1986-98 were depurated prior to analysis; depuration was discontinued in 1999. Arsenic was not analyzed until 2003; therefore, the number of samples may be small or zero for some taxa. Values are reported using U.S. Geological Survey rounding standards. Abbreviation: spp., one or more similar species. Symbols: $<$, less than minimum reporting level; --, indicates either too few samples (less than three) or insufficient data to compute statistic, or element not analyzed]

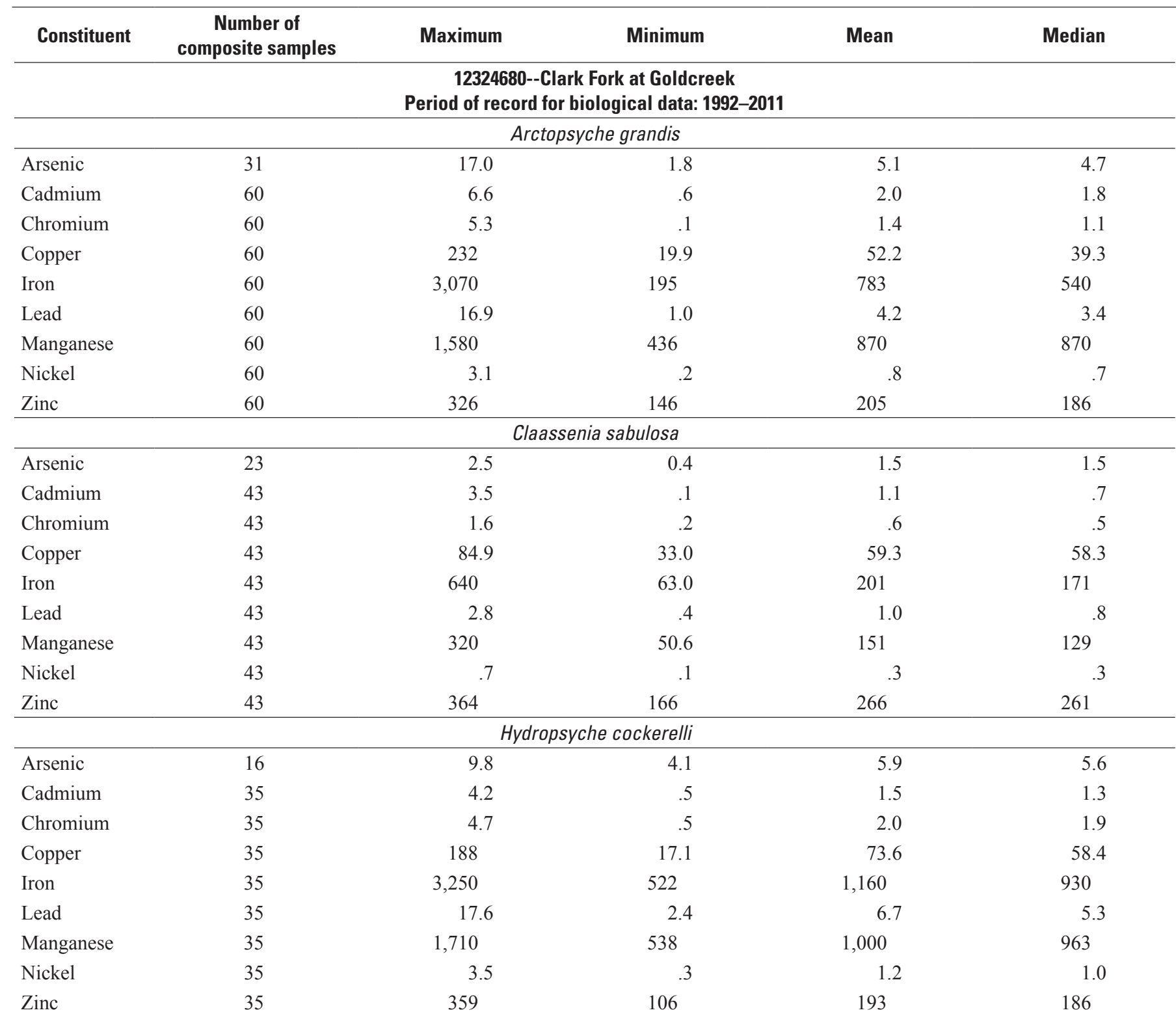


Table 27. Statistical summary of long-term biological data for the Clark Fork basin, Montana, August 1986 through August 2011.-Continued

[Concentrations are in micrograms per gram dry weight $(\mu \mathrm{g} / \mathrm{g})$. Number of composite samples represents the total of all individual composite samples collected for every year that the constituent was analyzed. Values for a single sample are arbitrarily listed in the "Mean" column. Because Hydropsyche insects were not sorted to the species level during 1986-89, Hydropsyche species statistics for stations sampled during those years are based on the results of all Hydropsyche species combined. At some sites, statistics of Hydropsyche morosa group are based on the combined results of two or more species. Insects collected during 1986-98 were depurated prior to analysis; depuration was discontinued in 1999. Arsenic was not analyzed until 2003; therefore, the number of samples may be small or zero for some taxa. Values are reported using U.S. Geological Survey rounding standards. Abbreviation: spp., one or more similar species. Symbols: $<$, less than minimum reporting level; --, indicates either too few samples (less than three) or insufficient data to compute statistic, or element not analyzed]

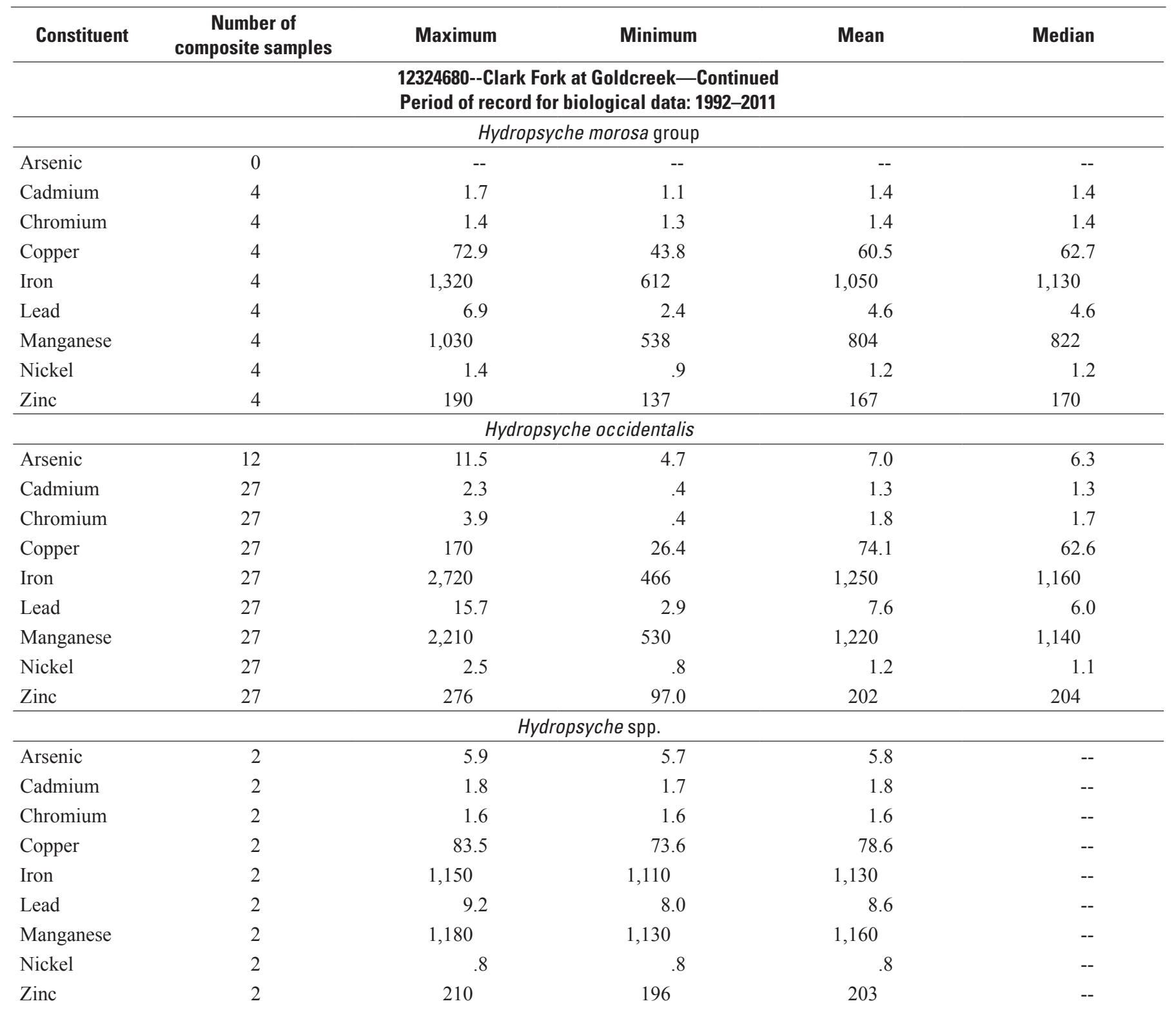


Table 27. Statistical summary of long-term biological data for the Clark Fork basin, Montana, August 1986 through August 2011.-Continued

[Concentrations are in micrograms per gram dry weight $(\mu \mathrm{g} / \mathrm{g})$. Number of composite samples represents the total of all individual composite samples collected for every year that the constituent was analyzed. Values for a single sample are arbitrarily listed in the "Mean" column. Because Hydropsyche insects were not sorted to the species level during 1986-89, Hydropsyche species statistics for stations sampled during those years are based on the results of all Hydropsyche species combined. At some sites, statistics of Hydropsyche morosa group are based on the combined results of two or more species. Insects collected during 1986-98 were depurated prior to analysis; depuration was discontinued in 1999. Arsenic was not analyzed until 2003; therefore, the number of samples may be small or zero for some taxa. Values are reported using U.S. Geological Survey rounding standards. Abbreviation: spp., one or more similar species. Symbols: $<$, less than minimum reporting level; --, indicates either too few samples (less than three) or insufficient data to compute statistic, or element not analyzed]

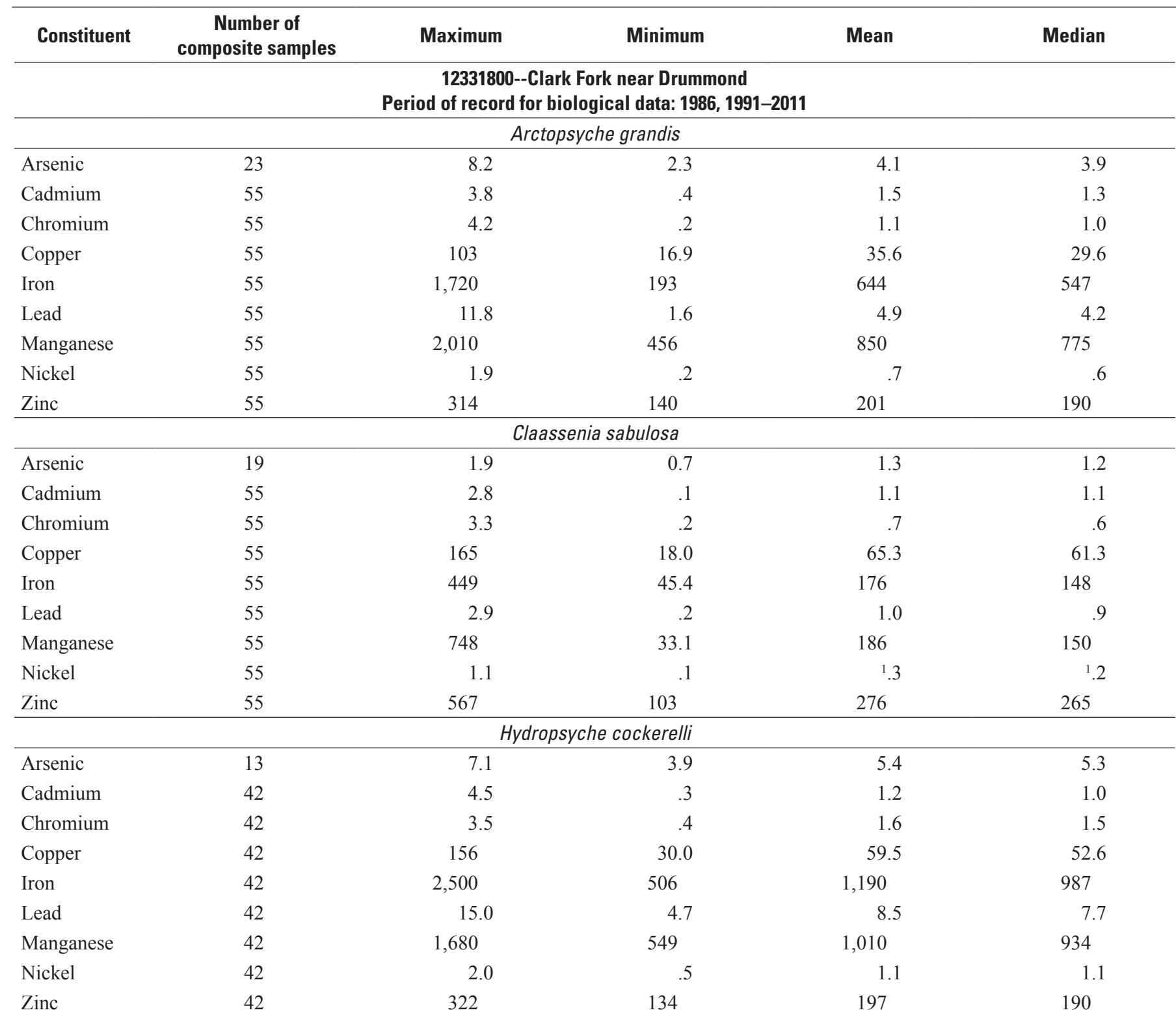


Table 27. Statistical summary of long-term biological data for the Clark Fork basin, Montana, August 1986 through August 2011.-Continued

[Concentrations are in micrograms per gram dry weight $(\mu \mathrm{g} / \mathrm{g})$. Number of composite samples represents the total of all individual composite samples collected for every year that the constituent was analyzed. Values for a single sample are arbitrarily listed in the "Mean" column. Because Hydropsyche insects were not sorted to the species level during 1986-89, Hydropsyche species statistics for stations sampled during those years are based on the results of all Hydropsyche species combined. At some sites, statistics of Hydropsyche morosa group are based on the combined results of two or more species. Insects collected during 1986-98 were depurated prior to analysis; depuration was discontinued in 1999. Arsenic was not analyzed until 2003; therefore, the number of samples may be small or zero for some taxa. Values are reported using U.S. Geological Survey rounding standards. Abbreviation: spp., one or more similar species. Symbols: $<$, less than minimum reporting level; --, indicates either too few samples (less than three) or insufficient data to compute statistic, or element not analyzed]

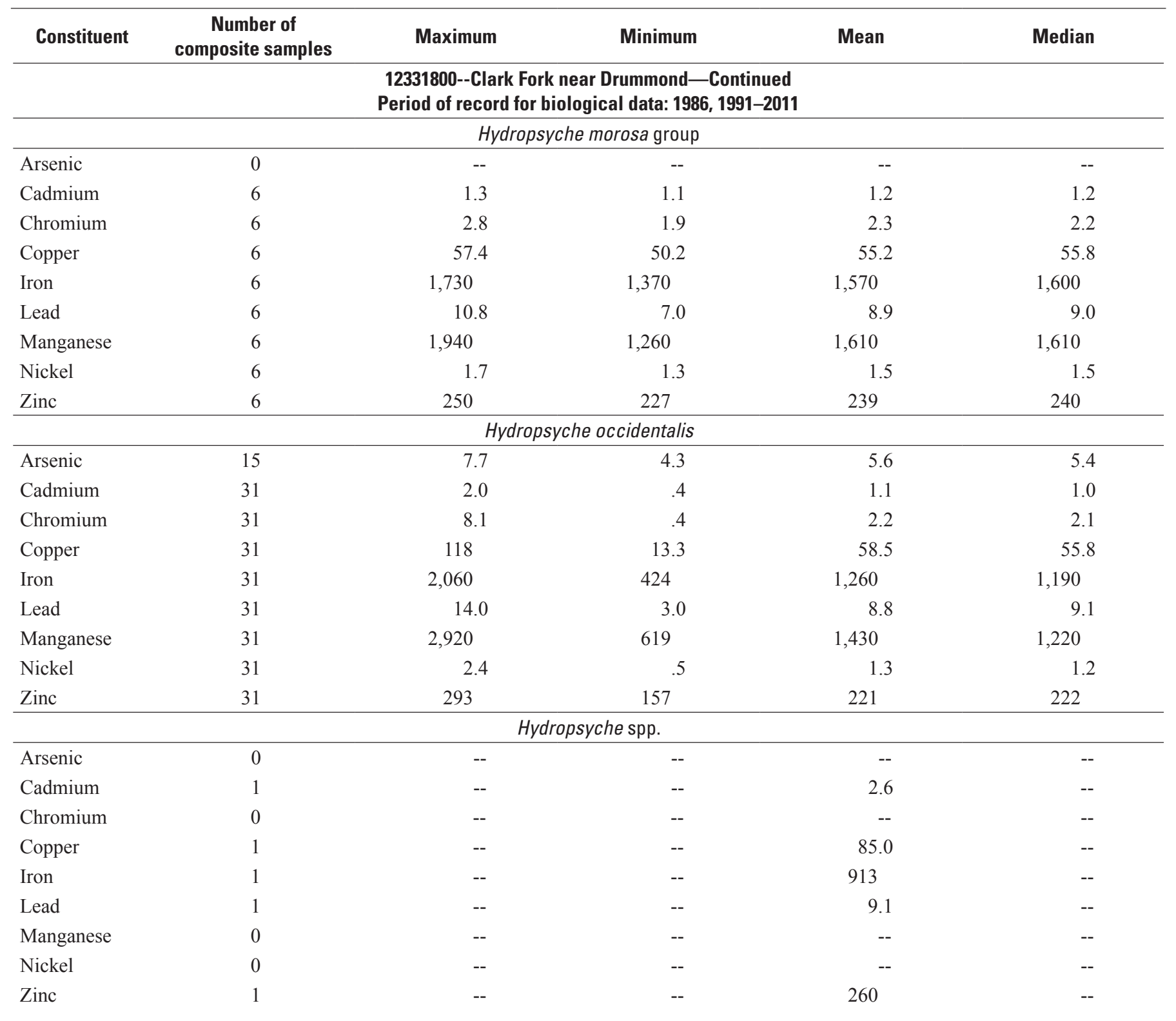


Table 27. Statistical summary of long-term biological data for the Clark Fork basin, Montana, August 1986 through August 2011.-Continued

[Concentrations are in micrograms per gram dry weight $(\mu \mathrm{g} / \mathrm{g})$. Number of composite samples represents the total of all individual composite samples collected for every year that the constituent was analyzed. Values for a single sample are arbitrarily listed in the "Mean" column. Because Hydropsyche insects were not sorted to the species level during 1986-89, Hydropsyche species statistics for stations sampled during those years are based on the results of all Hydropsyche species combined. At some sites, statistics of Hydropsyche morosa group are based on the combined results of two or more species. Insects collected during 1986-98 were depurated prior to analysis; depuration was discontinued in 1999. Arsenic was not analyzed until 2003; therefore, the number of samples may be small or zero for some taxa. Values are reported using U.S. Geological Survey rounding standards. Abbreviation: spp., one or more similar species. Symbols: $<$, less than minimum reporting level; --, indicates either too few samples (less than three) or insufficient data to compute statistic, or element not analyzed]

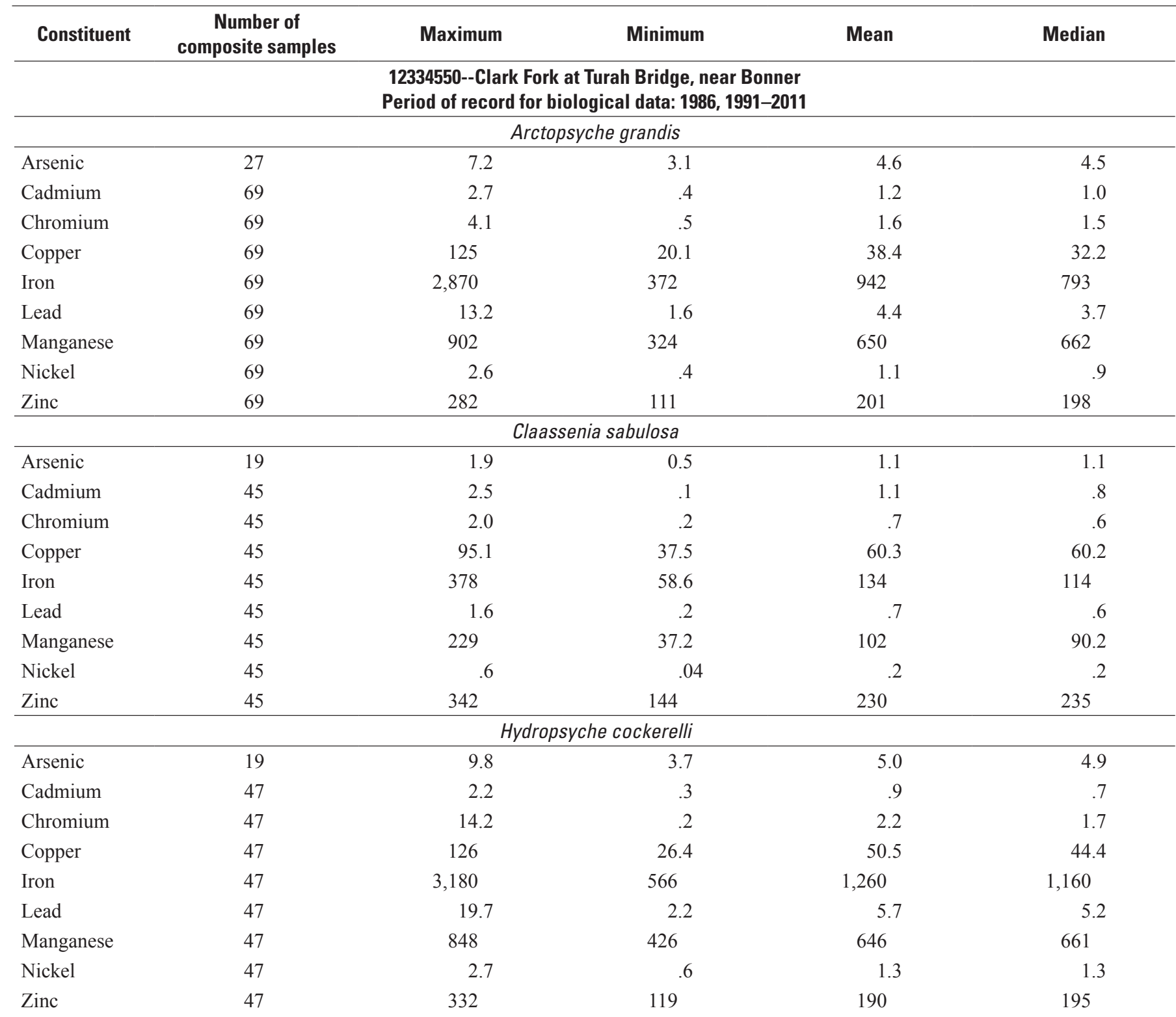


Table 27. Statistical summary of long-term biological data for the Clark Fork basin, Montana, August 1986 through August 2011.-Continued

[Concentrations are in micrograms per gram dry weight $(\mu \mathrm{g} / \mathrm{g})$. Number of composite samples represents the total of all individual composite samples collected for every year that the constituent was analyzed. Values for a single sample are arbitrarily listed in the "Mean" column. Because Hydropsyche insects were not sorted to the species level during 1986-89, Hydropsyche species statistics for stations sampled during those years are based on the results of all Hydropsyche species combined. At some sites, statistics of Hydropsyche morosa group are based on the combined results of two or more species. Insects collected during 1986-98 were depurated prior to analysis; depuration was discontinued in 1999. Arsenic was not analyzed until 2003; therefore, the number of samples may be small or zero for some taxa. Values are reported using U.S. Geological Survey rounding standards. Abbreviation: spp., one or more similar species. Symbols: $<$, less than minimum reporting level; --, indicates either too few samples (less than three) or insufficient data to compute statistic, or element not analyzed]

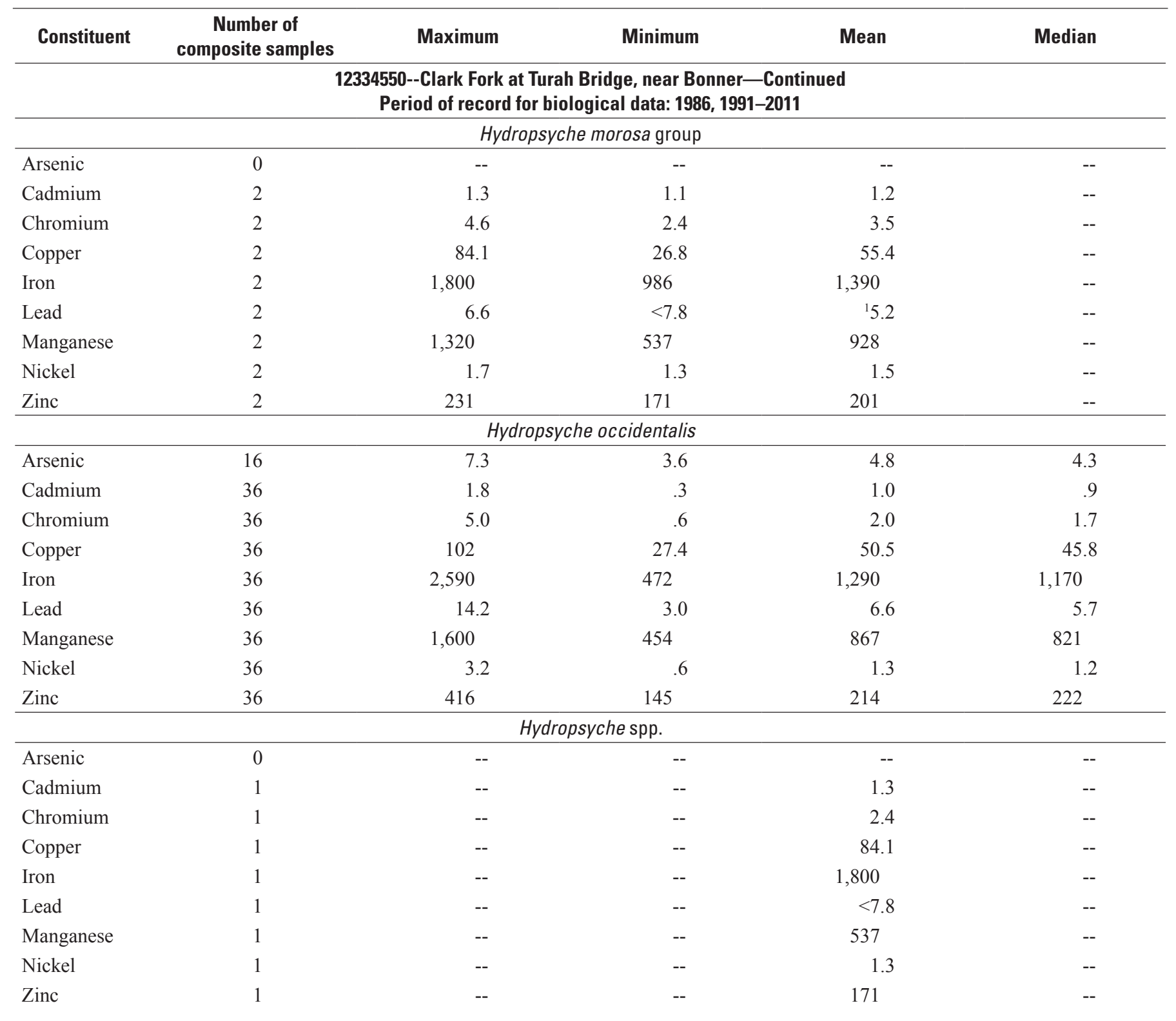


Table 27. Statistical summary of long-term biological data for the Clark Fork basin, Montana, August 1986 through August 2011.-Continued

[Concentrations are in micrograms per gram dry weight $(\mu \mathrm{g} / \mathrm{g})$. Number of composite samples represents the total of all individual composite samples collected for every year that the constituent was analyzed. Values for a single sample are arbitrarily listed in the "Mean" column. Because Hydropsyche insects were not sorted to the species level during 1986-89, Hydropsyche species statistics for stations sampled during those years are based on the results of all Hydropsyche species combined. At some sites, statistics of Hydropsyche morosa group are based on the combined results of two or more species. Insects collected during 1986-98 were depurated prior to analysis; depuration was discontinued in 1999. Arsenic was not analyzed until 2003; therefore, the number of samples may be small or zero for some taxa. Values are reported using U.S. Geological Survey rounding standards. Abbreviation: spp., one or more similar species. Symbols: $<$, less than minimum reporting level; --, indicates either too few samples (less than three) or insufficient data to compute statistic, or element not analyzed]

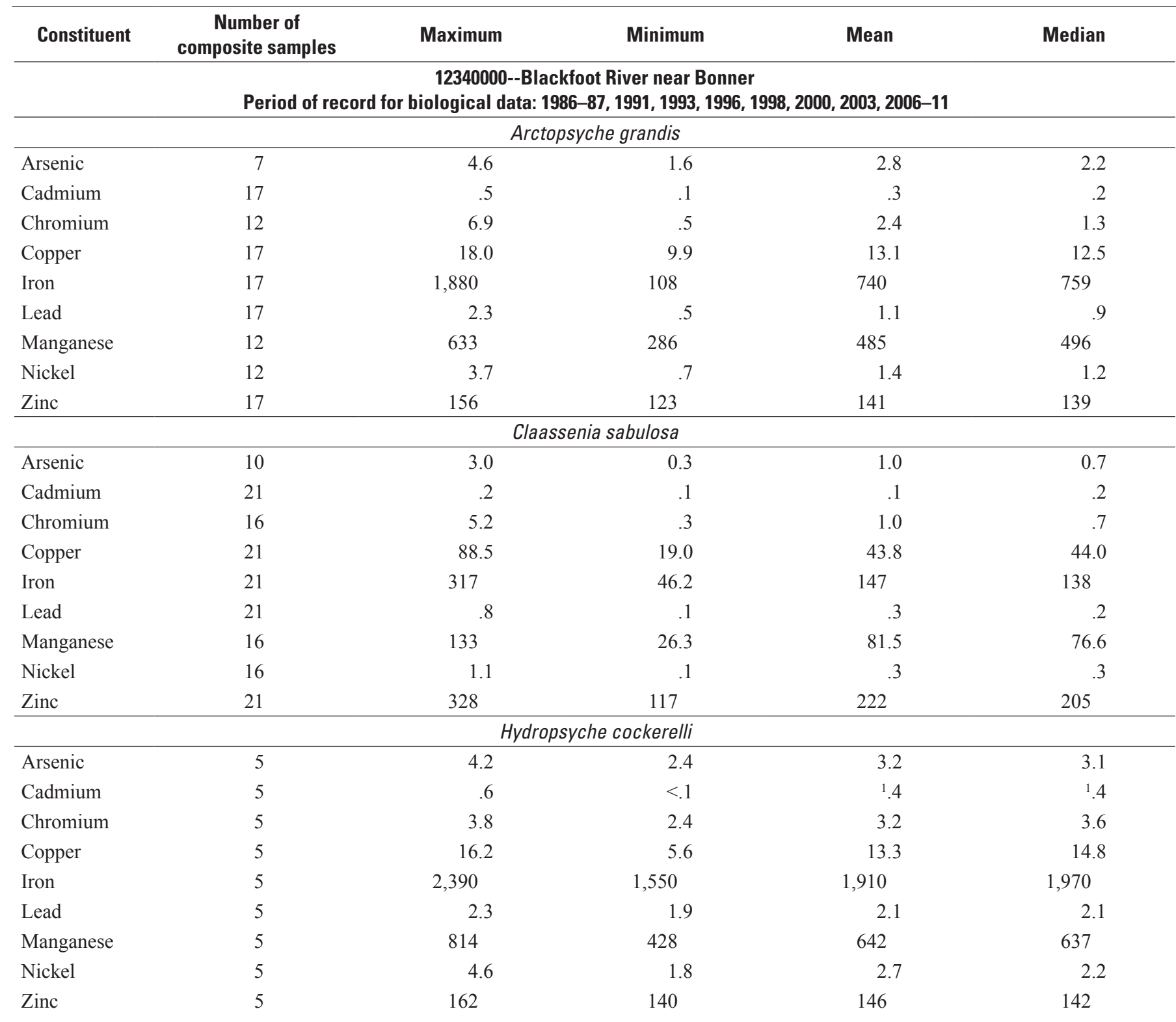


Table 27. Statistical summary of long-term biological data for the Clark Fork basin, Montana, August 1986 through August 2011.-Continued

[Concentrations are in micrograms per gram dry weight $(\mu \mathrm{g} / \mathrm{g})$. Number of composite samples represents the total of all individual composite samples collected for every year that the constituent was analyzed. Values for a single sample are arbitrarily listed in the "Mean" column. Because Hydropsyche insects were not sorted to the species level during 1986-89, Hydropsyche species statistics for stations sampled during those years are based on the results of all Hydropsyche species combined. At some sites, statistics of Hydropsyche morosa group are based on the combined results of two or more species. Insects collected during 1986-98 were depurated prior to analysis; depuration was discontinued in 1999. Arsenic was not analyzed until 2003; therefore, the number of samples may be small or zero for some taxa. Values are reported using U.S. Geological Survey rounding standards. Abbreviation: spp., one or more similar species. Symbols: $<$, less than minimum reporting level; --, indicates either too few samples (less than three) or insufficient data to compute statistic, or element not analyzed]

\begin{tabular}{|c|c|c|c|c|c|}
\hline Constituent & $\begin{array}{c}\text { Number of } \\
\text { composite samples }\end{array}$ & Maximum & Minimum & Mean & Median \\
\hline \multicolumn{6}{|c|}{$\begin{array}{l}\text { 12340000--Blackfoot River near Bonner-Continued } \\
\text { Period of record for biological data: 1986-87, 1991, 1993, 1996, 1998, 2000, 2003, 2006-11 }\end{array}$} \\
\hline \multicolumn{6}{|c|}{ Hydropsyche occidentalis } \\
\hline Arsenic & 10 & 3.8 & 1.2 & 2.4 & 2.2 \\
\hline Cadmium & 22 & .5 & .1 & .2 & .2 \\
\hline Copper & 22 & 20.6 & 12.0 & 15.7 & 15.1 \\
\hline Iron & 22 & 2,090 & 1,010 & 1,530 & 1,500 \\
\hline Lead & 22 & 2.0 & .8 & 1.5 & 1.6 \\
\hline Manganese & 22 & 798 & 414 & 529 & 470 \\
\hline Nickel & 22 & 4.9 & .9 & 1.6 & 1.4 \\
\hline Cadmium & 1 & -- & -- & 0.6 & -- \\
\hline Chromium & 1 & -- & -- & 1.6 & -- \\
\hline Copper & 1 & -- & -- & 13.9 & -- \\
\hline Iron & 1 & -- & -- & 1,140 & -- \\
\hline Lead & 1 & -- & -- & 2.9 & -- \\
\hline Manganese & 1 & -- & -- & 525 & -- \\
\hline Nickel & 1 & -- & -- & 2.8 & -- \\
\hline Zinc & 1 & -- & -- & 132 & -- \\
\hline
\end{tabular}


Table 27. Statistical summary of long-term biological data for the Clark Fork basin, Montana, August 1986 through August 2011.-Continued

[Concentrations are in micrograms per gram dry weight $(\mu \mathrm{g} / \mathrm{g})$. Number of composite samples represents the total of all individual composite samples collected for every year that the constituent was analyzed. Values for a single sample are arbitrarily listed in the "Mean" column. Because Hydropsyche insects were not sorted to the species level during 1986-89, Hydropsyche species statistics for stations sampled during those years are based on the results of all Hydropsyche species combined. At some sites, statistics of Hydropsyche morosa group are based on the combined results of two or more species. Insects collected during 1986-98 were depurated prior to analysis; depuration was discontinued in 1999. Arsenic was not analyzed until 2003; therefore, the number of samples may be small or zero for some taxa. Values are reported using U.S. Geological Survey rounding standards. Abbreviation: spp., one or more similar species. Symbols: $<$, less than minimum reporting level; --, indicates either too few samples (less than three) or insufficient data to compute statistic, or element not analyzed]

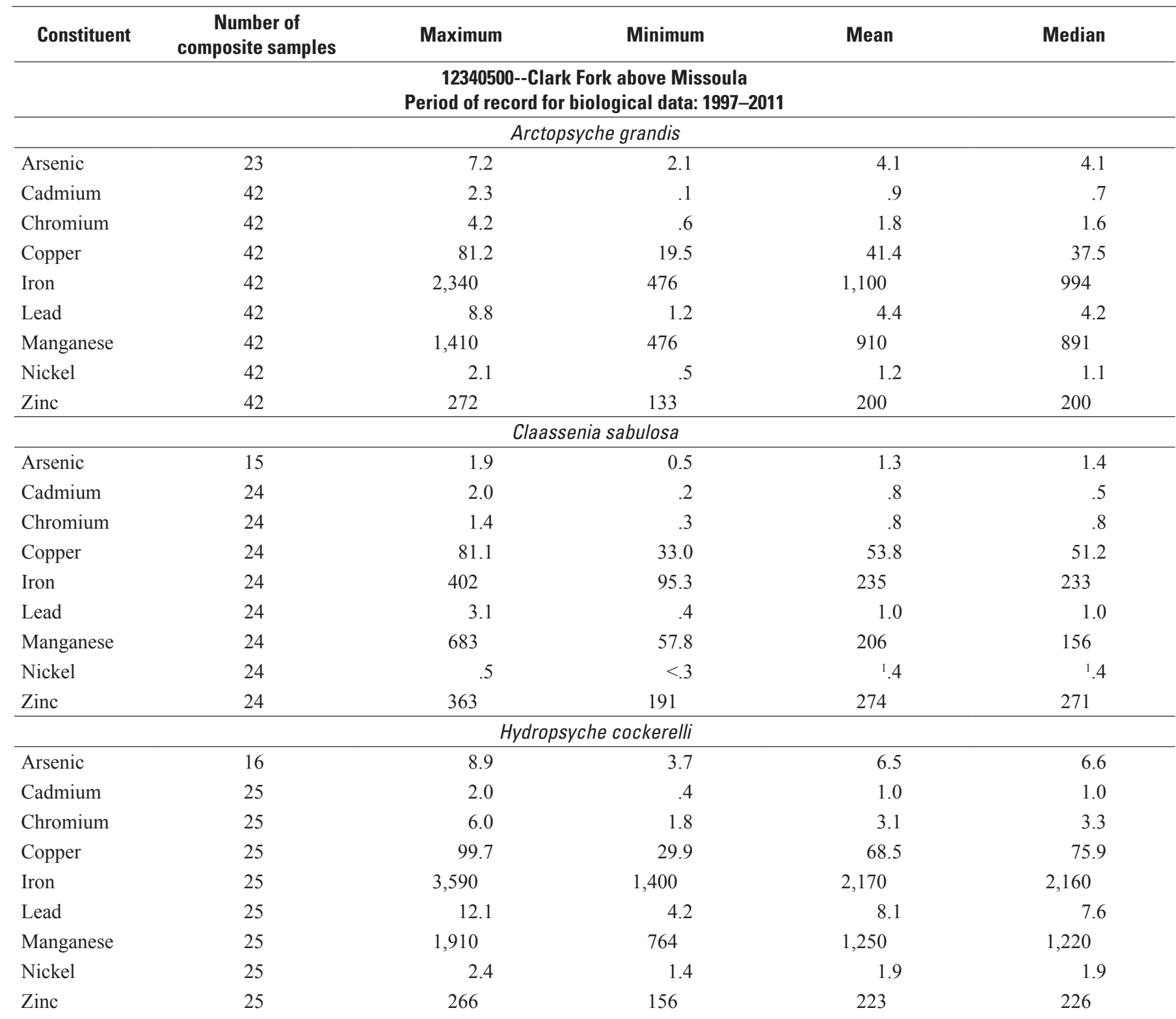


Table 27. Statistical summary of long-term biological data for the Clark Fork basin, Montana, August 1986 through August 2011.-Continued

[Concentrations are in micrograms per gram dry weight $(\mu \mathrm{g} / \mathrm{g})$. Number of composite samples represents the total of all individual composite samples collected for every year that the constituent was analyzed. Values for a single sample are arbitrarily listed in the "Mean" column. Because Hydropsyche insects were not sorted to the species level during 1986-89, Hydropsyche species statistics for stations sampled during those years are based on the results of all Hydropsyche species combined. At some sites, statistics of Hydropsyche morosa group are based on the combined results of two or more species. Insects collected during 1986-98 were depurated prior to analysis; depuration was discontinued in 1999. Arsenic was not analyzed until 2003; therefore, the number of samples may be small or zero for some taxa. Values are reported using U.S. Geological Survey rounding standards. Abbreviation: spp., one or more similar species. Symbols: $<$, less than minimum reporting level; --, indicates either too few samples (less than three) or insufficient data to compute statistic, or element not analyzed]

\begin{tabular}{|c|c|c|c|c|c|}
\hline Constituent & $\begin{array}{c}\text { Number of } \\
\text { composite samples }\end{array}$ & Maximum & Minimum & Mean & Median \\
\hline \multicolumn{6}{|c|}{$\begin{array}{l}\text { 12340500--Clark Fork above Missoula-Continued } \\
\text { Period of record for biological data: 1997-2011 }\end{array}$} \\
\hline \multicolumn{6}{|c|}{ Hydropsyche occidentalis } \\
\hline Arsenic & 9 & 7.4 & 3.9 & 5.8 & 6.2 \\
\hline Cadmium & 15 & 1.5 & .4 & .9 & .8 \\
\hline Copper & 15 & 80.7 & 30.3 & 59.2 & 59.5 \\
\hline Iron & 15 & 2,540 & 1,450 & 2,080 & 2,210 \\
\hline Lead & 15 & 11.4 & 4.0 & 7.4 & 7.1 \\
\hline Manganese & 15 & 2,470 & 718 & 1,620 & 1,600 \\
\hline Nickel & 15 & 2.4 & 1.4 & 1.9 & 1.9 \\
\hline
\end{tabular}

${ }^{1}$ Values determined by substituting one-half of the minimum reporting level for censored $(<)$ values when both uncensored and censored values were used in determining the mean and median. When all data were less than the minimum reporting level, the median was determined by ranking the censored values in order of detection. 
Publishing support provided by the: Denver Publishing Service Center

For more information concerning this publication, contact: Director, Montana Water Science Center U.S. Geological Survey

3162 Bozeman Ave.

Helena, MT 59601

(406) 457-5900

Or visit the Montana Water Science Center Web site at: http://mt.water.usgs.gov/ 


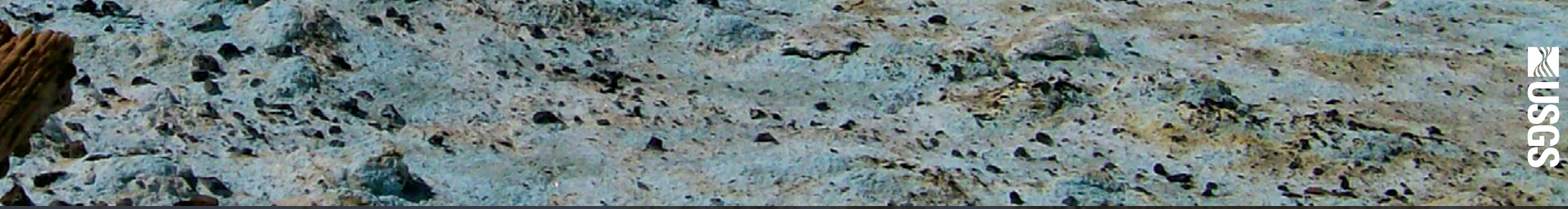
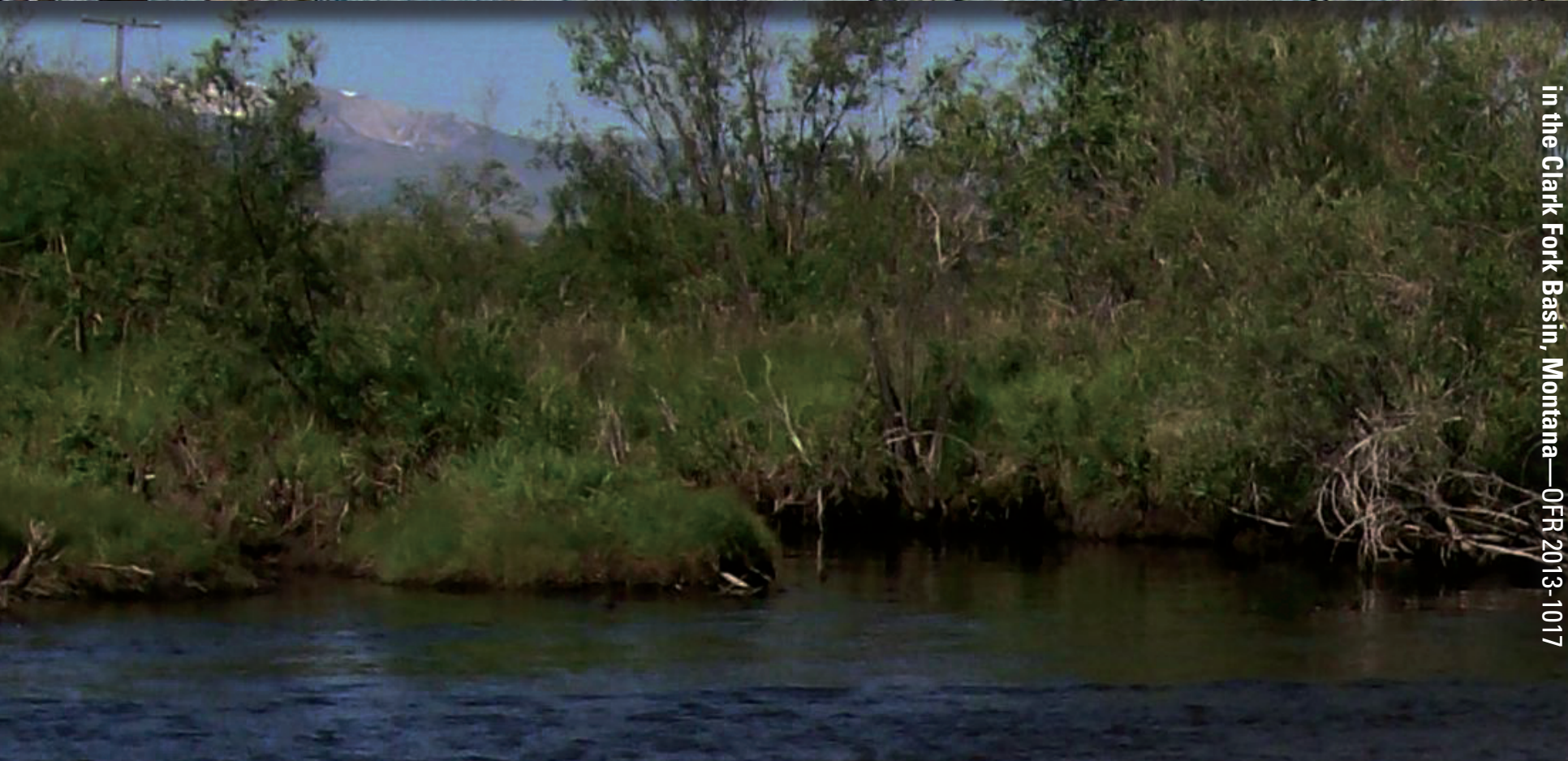

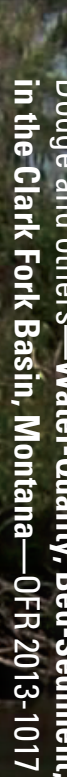
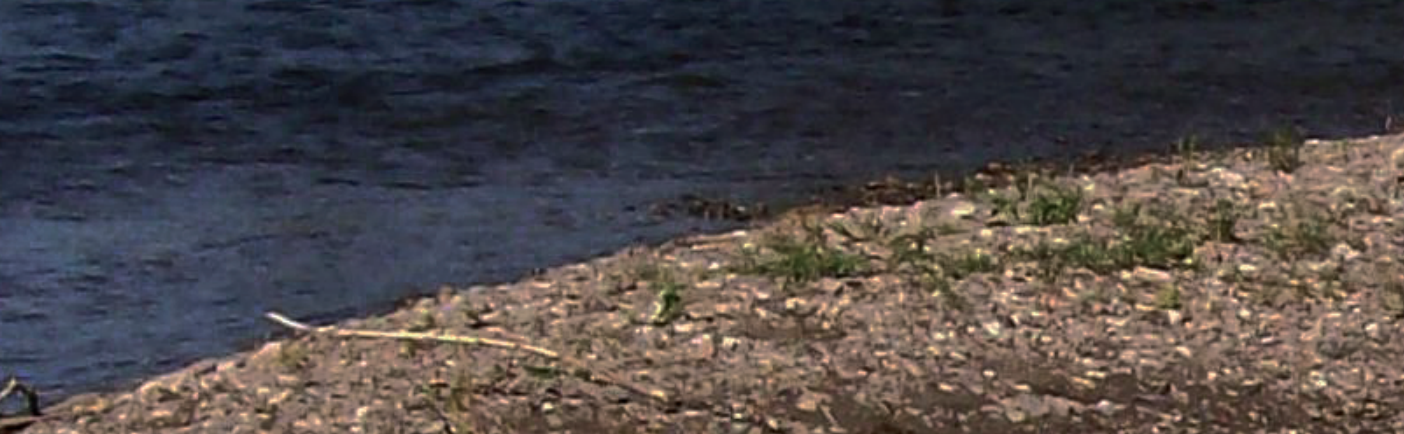

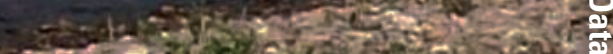
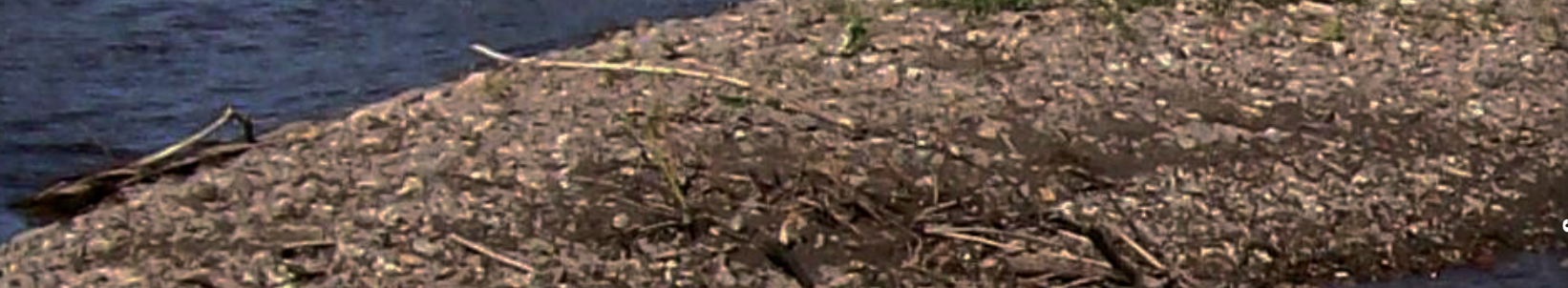

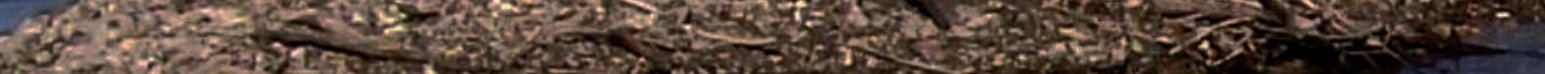

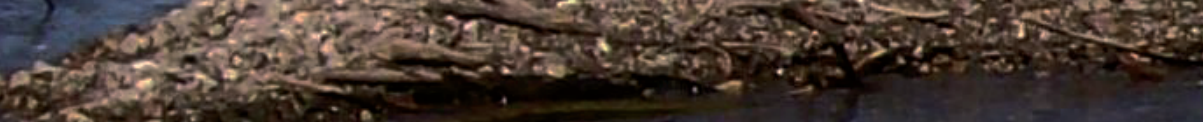

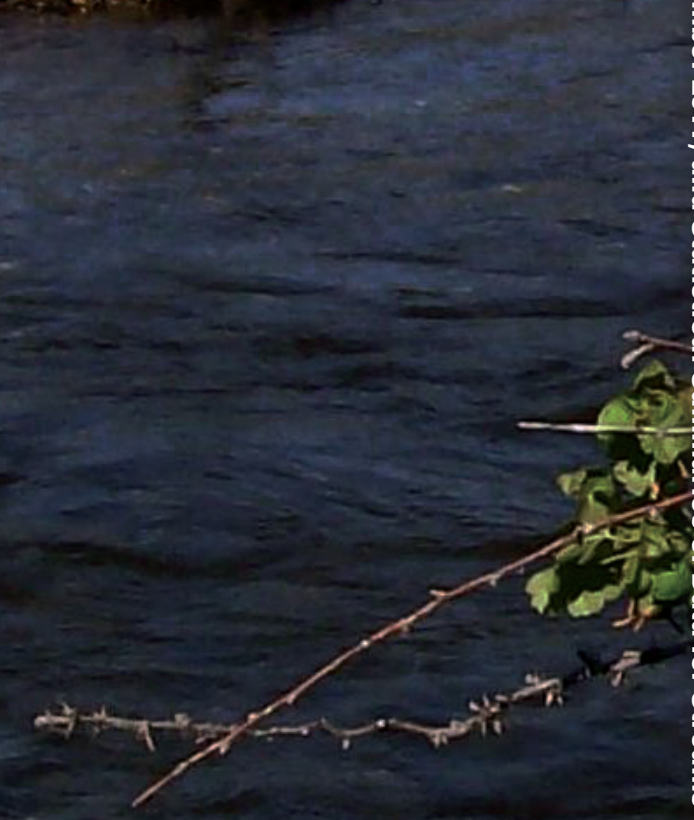

